\title{
Office of Military Government for Germany (U.S.)
}

\author{
Von
}

Josef Henke und Klaus Oldenhage 



\section{A. Historischer Überblick Verwaltungsgeschichte der amerikanischen Militärregierung in Deutschland im Überblick (1944-1949)}

\section{Alliierte Kriegszielplanungen}

Hitlers Angriff auf die Sowjetunion und die Kriegserklärung der Vereinigten Staaten von Amerika stellten diese beiden Mächte und GroBbritannien seit 1941 vor die Aufgabe, eine gemeinsame Politik gegenüber Deutschland zu entwickeln.' Am 14. August 1941 formulierten der amerikanische Präsident Franklin D. Roosevelt und der britische Premierminister Winston S. Churchill in der Atlantik-Charta die alliierten Kriegsziele, deren Hauptforderung darin bestand, „daB alle Menschen in allen Ländern der Welt ihr Leben frei von Furcht und Mangel leben könnten “. ${ }^{2}$ Die Vereinigten Staaten und GroBbritannien verzichteten auf territorialen Erwerb und wandten sich gegen jede Gebietsabtretung, die nicht den Wünschen der Betroffenen entsprach. ${ }^{3}$ In Erklärungen vom 24. September 1941 und 1.Januar 1942 akzeptierten alle Staaten, die gegen Deutschland und die mit ihm verbündeten Măchte im Kriege standen, die Grundsätze der Atlantik-Charta; die Sowjetunion machte allerdings den Vorbehalt, daß die praktische Anwendung der Prinzipien notwendigerweise an die Umstände, Bedürfnisse und historischen Eigenarten der jeweiligen Länder angepaßt werden müsse. Deutschland sollte sich jedoch nicht auf die Atlantik-Charta berufen können. ${ }^{4}$

Den Plan einer Zerstückelung des Deutschen Reiches hatte Josef Stalin erstmals in einem Gespräch mit dem britischen Außenminister Anthony Eden im Dezember 1941 in die Diskussion eingefuhrt. ${ }^{5}$ Im Januar 1943 proklamierten Roosevelt und Churchill in Casablanca die Forderung nach bedingungsloser Kapitulation Deutschlands. Dies wurde auf der Außenministerkonferenz in Moskau im Oktober 1943 ausdrucklich bestätigt; man beschloB, die Zusammenarbeit der drei Mächte auch nach Beendigung der Feindseligkeiten fortzusetzen, und verständigte sich auf einige Grundzüge der Deutschlandpolitik: Das Gebiet des Deutschen Reiches wurde als Territorium innerhalb der Grenzen vom 1. Januar 1937 definiert.

Österreich und alle anderen nach dem genannten Zeitpunkt annektierten Gebiete ${ }^{6}$ würden also nach der Kapitulation nicht mehr zu Deutschland gehören, das von sowjetischen, britischen und amerikanischen Truppen besetzt werden sollte. Die Regierungsgewalt in Deutschland wollte man einer interalliierten Kontrollkommission ubertragen. Deren vordringliche Aufgabe sollte in der Entmilitarisierung, Entnazifizierung und Demokratisierung des deutschen Volkes liegen. Die deutsche Kriegsindu-

Eine Sammlung der General Orders von USGCC und von OMGUS befindet sich sowohl im Bundesarchiv (Z 45F) als auch im Archiv des Instituts für Zeitgeschichte (MA 1417). Im folgenden wird auf die Fundortangabe verzichtet.

1 Vgl. zusammenfassend Graml: Die deutsche Frage, S. $281 \mathrm{f}$.

${ }^{2}$ Erdmann: Zeit der Weltkriege, S. $595 \mathrm{ff}$.

${ }^{3}$ Graml: Die deutsche Frage, S. 282.

${ }^{4}$ Erdmann: Zeit der Weltkriege, S. 596.

5 Backer: Entscheidung zur Teilung, S.23ff.

6 Vgl. Oldenhage: Verwaltung der besetzten Gebiete, S.1131 ff. 
strie sollte zerstört, der von Deutschland angerichtete Schaden durch Reparationsleistungen wiedergutgemacht werden. ${ }^{7}$ Zur Ausarbeitung von Einzelheiten eines solchen Programmes setzte man eine Europäische Beratende Kommission (European Advisory Commission) ${ }^{8}$ ein, die aus dem amerikanischen und dem sowjetischen Botschafter in London (John G. Winant und Fedor T. Gousev) sowie einem Beamten des britischen Außenministeriums (Sir William Strang) bestand und im Januar 1944 ihre Arbeit in London aufnahm; ab Ende 1944 war auch Frankreich dort vertreten. ${ }^{9}$

Als sich Roosevelt, Stalin und Churchill um die Monatswende November/Dezember 1943 in Teheran trafen, zeigte es sich, wie stark die deutsche mit der polnischen Frage verknüpft war; der Verlust der deutschen Ostgebiete jenseits von Oder und Neiße zeichnete sich bereits ebenso deutlich ab wie Konturen eines zerstückelten Deutschlands. Roosevelt dachte daran, das Deutsche Reich in fünf selbständige Staaten zu tejlen und weitere Gebiete unter internationale Verwaltung zu stellen. Stalin sicherte sich vor allem die politische Zustimmung der Westalliierten für seine Pläne der Verlagerung Polens nach Westen und der sowjetischen Annexion des Gebietes um Königsberg. ${ }^{10}$ Die Europäische Beratende Kommission wurde mit der Ausarbeitung von Waffenstillstandsbedingungen sowie der Vorbereitung der Besetzung und Verwaltung Deutschlands durch die alliierten Mächte beauftragt. Auch auf allen folgenden internationalen Kriegskonferenzen wurde die Zerstückelung Deutschlands intensiv beraten, ohne daß es jedoch zu förmlichen Beschlüssen gekommen wäre. Nach der Konferenz in Teheran entbrannte in den Vereinigten Staaten von Amerika ein lebhafter Streit um die Zukunft Deutschlands, der mit dem Stichwort „Morgenthau-Plan“ (Rückverwandlung Deutschlands in einen Agrarstaat) gekennzeichnet werden kann.

In zwei Abkommen vom 12. September und 14. November 1944 einigten sich die Alliierten über die Grenzen der Besatzungszonen der drei Mächte, die Einrichtung eines Alliierten Kontrollrats sowie die gemeinsame Besetzung und Verwaltung von Berlin." Die Gebiete, die unter sowjetische und polnische Verwaltung fallen sollten (OstpreuBen, Schlesien und Teile von Pommern und Brandenburg) waren bei der Einteilung in Besatzungszonen schon nicht mehr berücksichtigt worden. Die sowjetisch besetzten Gebiete im Osten waren der Fläche nach fast genau so groß wie die von Engländern und Amerikanern zu besetzenden Zonen im Nordwesten und Südwesten Deutschlands. Die Demarkationslinie zwischen den beiden westlichen Zonen und dem sowjetischen Anteil entsprach der späteren Grenze zwischen der Bundesrepublik Deutschland und der Deutschen Demokratischen Republik. Berlin sollte in Sektoren geteilt als besonderes Territorium durch eine gemeinsame "Kommandatura" von den Alliierten verwaltet werden. Schließlich kam man überein, die Militärgouverneure der Besatzungszonen für Angelegenheiten Gesamtdeutschlands gemeinsam verantwortlich zu machen und ihnen als Lenkungsorgan eine Alliierte Kontrollbehörde in Berlin zur Verfügung zu stellen. ${ }^{12}$ Politische Entscheidungen über das Schicksal Deutschlands waren damit indes nicht verbunden.

\footnotetext{
${ }^{7}$ Benz: Von der Besatzungsherrschaft, S. 20.

8 Vgl. Kowalski: European Advisory Commission, S. $261 \mathrm{ff}$.

${ }^{9}$ Benz: Von der Besatzungsherrschaft, S. 20.

${ }^{10} \mathrm{Vgl}$. K.-D. Henke: Weg nach Potsdam, S. $49 \mathrm{ff}$.

11 Foreign Relations, Malta and Yalta, S. $124 \mathrm{ff}$; Erdmann: Zeit der Weltkriege, S. 599. Vgl. auch USGCC 1944-45/1-3.

12 Benz: Von der Besatzungsherrschaft, S.21.
} 
Über die Zerstückelung Deutschlands wurde erneut auf der Konferenz der „Großen Drei" in Jalta vom 4. bis 11. Februar 1945 diskutiert. ${ }^{13}$ Während Stalin forderte, daß in die von der Europäischen Beratenden Kommission vorbereitete Kapitulationsurkunde auch eine Aussage über die Teilung Deutschlands aufgenommen werden sollte, war es Churchill zuzuschreiben, daß die Erklärung in dieser Frage eine klare Aussage vermied: Die Alliierten wollten sich hinsichtlich der Aufteilung Deutschlands solche Maßnahmen vorbehalten, die sie für einen künftigen Frieden und die Sicherheit für notwendig hielten. Konkrete territoriale Entscheidungen wurden nicht getroffen, die weitere Bearbeitung des Problems vielmehr einem neu gebildeten AusschuB für die deutsche Teilungsfrage überwiesen. ${ }^{14}$ Churchill, der mit einem baldigen Abzug der Amerikaner aus Europa rechnete, setzte es in Jalta durch, daß Frankreich an der künftigen Besatzung und an der Alliierten Kontrollbehörde für Deutschland beteiligt werden sollte. Es gelang ihm jedoch infolge des Widerstandes Stalins nicht, dem französischen Verbündeten auch einen Sitz im Ausschuß für die deutsche Teilungsfrage zu verschaffen. Im Interesse der Reparationen, über deren Form man sich in Jalta einig wurde ${ }^{15}$ schien es den Alliierten vorerst erforderlich, Deutschland als wirtschaftliche Einheit zu betrachten. Demontagen und die Verwendung deutscher Arbeitskräfte als Reparationsleistungen wurden vereinbart, doch blieb die Höhe der Reparationen offen. Insgesamt hatten in Jalta weltpolitische Probleme, insbesondere die Niederwerfung Japans, eine größere Rolle gespielt als Einzelheiten der deutschen Frage und der Niederwerfung des Nationalsozialismus. ${ }^{16}$

\section{Die Vorbereitung der amerikanischen Besatzung in Deutschland}

Im Unterschied zu den amerikanischen Truppen, die nach dem Ersten Weltkrieg Teile des Rheinlandes besetzt hatten, ${ }^{17}$ waren die amerikanischen Offiziere und Soldaten während des Zweiten Weltkrieges auf ihre künftige Aufgabe in Deutschland und anderen zu besetzenden Gebieten intensiv vorbereitet worden. Im April 1942 wurde eine School for Military Government an der Universität von Virginia in Charlottesville unter Leitung von Brigadegeneral Cornelius W. Wickersham errichtet. ${ }^{18} \mathrm{Im}$ amerikanischen Kriegsministerium (War Department) beschäftigten sich seit August 1942 eine Military Government Division in the Office of Provost Marshal General, der für Angelegenheiten der Militärpolizei zuständig war, und eine am 1. März 1943 im Generalstab errichtete Civil Affairs Division mit der Vorbereitung der Besetzung Deutschlands. ${ }^{19}$ Während sich das Office of Provost Marshal General verständlicherweise auf seine eigentlichen militärischen Aufgaben konzentrierte, konnte sich die Civil Affairs Division unter Generalmajor John $\mathbf{H}$. Hilldring in Ermangelung anderer Zuständigkeit allen nicht im engeren Sinne militärischen Problemen der Besatzung widmen. Später gab es mindestens zehn weitere Civil Affairs Training Schools an amerikanischen Universitäten. Für die Mannschaftsdienstgrade wurden besondere Army Specialized Training Programs durchgeführt.

\footnotetext{
${ }^{13}$ Foreign Relations, Malta and Yalta, S. $124 \mathrm{ff}$.

${ }^{14}$ Benz: Von der Besatzungsherrschaft, S.22; Erdmann: Zeit der Weltkriege, S.600.

$15 \mathrm{Vgl}$. Neubeginn und Restauration, S. $51 \mathrm{f}$.

${ }^{16}$ Erdmann: Zeit der Weltkriege, S.601.

${ }^{17}$ Ziemke: The U.S. Army, S. 3; Latour/Vogelsang: Okkupation und Wiederaufbau, S. $28 \mathrm{ff}$.

${ }^{18}$ Ziemke: The U.S. Army, S.7.

${ }^{19}$ Ebenda, S. 17.
} 
Die Qualität der Ausbildung ist schwer zu beurteilen; jedenfalls waren die amerikanischen Soldaten für ihren Einsatz im European Theater of Operations (ETO) besser ausgebildet worden als für jede vergleichbare Spezialaufgabe zuvor. Unter dem Begriff des European Theater of Operations, der Ende 1941 gebräuchlich wurde, verstand man Europa von den britischen Inseln bis ins östliche Deutschland, von Skandinavien bis zu den Pyrenäen, zum Mittelmeerraum und bis zum Balkan. Bereits im Mai 1941 hatten die Vereinigten Staaten eine Special Observers Group in London errichtet, damit sie sich gemeinsam mit den Briten auf die Besetzung Deutschlands vorbereiten konnten. Im Januar 1942 wurde diese Gruppe Teil der United States Armed Forces in the British Isles. Am 8. Juni 1942 ubemahm das Hauptquartier des European Theater of Operations, US Army (ETOUSA) ${ }^{20}$ in London das Kommando uber alle amerikanischen Truppen in Europa, wobei es erst im Januar 1943 zur Bildung einer Civil Affairs Section, ETOUSA, kam. Im April 1943 wurde die amerikanisch-britische Zusammenarbeit durch Errichtung einer gemeinsamen Planungseinrichtung in London, des Chief of Staff Supreme Allied Command (COSSAC), verstärkt, bei dem am 23. August 1943 eine Civil Affairs Section unter einem britischen Offizier errichtet wurde. Zum Leiter des US Civil Affairs Staff, COSSAC, wurde damals Oberst Cornelius E. Ryan ernannt, der bereits seit Juli 1943 die Civil Affairs Section, ETOUSA, leitete. Diese bestand aus vier Planungseinheiten (Zivilbevölkerung, Militärregierung, Wirtschaft, Personal und Ausbildung) sowie einer besonderen Einheit Area Research, die sich mit den zu besetzenden Ländern intensiv vertraut zu machen hatte. Die Civil Affairs Division, COSSAC, bestand aus sechs Unterabteilungen (Recht, Finanzen, Versorgung, Inneres, Wirtschaft und Informationswesen).

Lange blieb unklar, welcher der beiden Einrichtungen die Aufgaben der Zivilverwaltung in Deutschland schließlich zufallen würden. Am 12. November 1943 ordnete die Civil Affairs Division im War Department in Washington an, daB COSSAC künftig für Fragen der Zivilverwaltung zuständig sei; die Civil Affairs Section, ETOUSA, ging in der 1. (später 12.) amerikanischen Heeresgruppe auf. ${ }^{21}$

An die Stelle von COSSAC trat im Januar 1944 die Einrichtung, unter deren Oberbefehl die Vereinigten Staaten von Amerika und Großbritannien den Krieg gegen Deutschland zu Ende führten: das Supreme Headquarters, Allied Expeditionary Forces (SHAEF) unter General Dwight D. Eisenhower, der gleichzeitig amerikanischer Oberbefehlshaber für das European Theater of Operations United States Army war. ${ }^{22}$ Die Stäbe von SHAEF und ETOUSA blieben jedoch getrennt. Aus der Civil Affairs Division, COSSAC, wurde die Abteilung G-5, SHAEF. Die fünf Abteilungen des Generalstabes von SHAEF (G-1 bis G-5) entsprachen im Grundsatz den entsprechenden Abteilungen des Vereinigten Generalstabs (Joint Chiefs of Staff [JCS]) im War Department in Washington:

$\begin{array}{ll}\text { G-1 } & \text { Personnel Division } \\ \text { G-2 } & \text { Military Intelligence Division } \\ \text { G-3 } & \text { Organization and Training Division } \\ \text { G-4 } & \text { Supply Division } \\ \text { G-5 } & \text { Civil Affairs Division }\end{array}$

${ }^{20}$ Zum folgenden ebenda, S. $24 \mathrm{ff}$.

${ }^{21}$ Ebenda, S.31.

22 Vgl. Steger: Clays Stabskonferenzen, S. $113 \mathrm{ff}$.; Latour/Vogelsang: Okkupation und Wiederaufbau, S.32f.; Ziemke: The U.S. Army, S. $42 \mathrm{ff}$.

${ }^{23}$ Vgl. ebenda, S. 458. 
Hinzu traten im Falle von SHAEF zahlreiche Sonderabteilungen, wie z. B.:

\author{
Adjutant General \\ Public Relations \\ Psychological Warfare \\ Signal \\ Engineer \\ Medical \\ Air Defense.
}

Bereits im Sommer 1942 hatte ETOUSA ein amerikanisches Schulungszentrum für künftige Angehörige der Militärregierung in Europa in Shrivenham, etwa $100 \mathrm{~km}$ westlich von London, errichtet. Ebenfalls nach Shrivenham legte man im Dezember 1943 ein Civil Affairs Center, das als Zentralstelle für die zivilen Aufgaben der künftigen regionalen und lokalen Militärregierungen (Military Government Detachments) fungierte. Im Januar 1944 wurde in Shrivenham aus etwa 150 amerikanischen und britischen Offizieren im Rahmen der dort aufgestellten European Civil Affairs Division eine German Country Section aufgebaut, die sich speziell auf einen Einsatz in Deutschland vorzubereiten hatte. Mitte Februar 1944 bildete G-5, SHAEF, schlieBlich eine Landeseinheit für Deutschland (German Country Unit, bzw. German Country Section) ${ }^{24}$ die später mit Recht von OMGUS-Chronisten als erste Vorläuferorganisation von OMGUS bezeichnet worden ist.

Die Verwaltungsstruktur der German Country Unit entsprach in etwa dem Aufbau der obersten deutschen Reichsbehörden. Dies läBt den SchluB zu, daB die Alliierten damals noch davon ausgingen, deutsche Regierungsstellen als Instrument ihrer Besatzungspolitik gebrauchen zu können. Die German Country Unit sollte die Besetzung Deutschlands sowohl im Verwaltungsaufbau - von der Reichs- bis zur Landkreis- und Gemeindeebene - als auch in allen Sachbereichen - Verschleppte, Wirtschaft, offentliche Einrichtungen, Ernährung, Arbeit, Post- und Fernmeldewesen, Finanzen, Justiz, Inneres, Verkehr, Vermögensverwaltung - umfassend vorbereiten. Dafür wurden intensive Ausbildungsprogramme für Offiziere in Schl lisselpositionen durchgefuhrt. ${ }^{25}$

Der Versuch, ein umfassendes Handbuch für die Besatzungsverwaltung verbindlich einzufuhren, war weit uber das Entwurfsstadium hinausgelangt, ${ }^{26}$ scheiterte jedoch offiziell am Widerspruch von Präsident Roosevelt, der sich mit den politischen Zielen der an den Grundsätzen der Atlantik-Charta orientierten Verfasser nicht mehr identifizieren wollte. Auf der anderen Seite war die Arbeit an diesem Handbuch nicht vergeblich gewesen, da nach einigen Änderungen durch G-5, SHAEF, der Entwurf inoffiziell herausgegeben und von der Militärregierung später intensiv genutzt wurde. Entsprechende Unterlagen stellte man auch fur bestimmte Fachverwaltungen zusammen. ${ }^{27}$ Nach den alliierten Landungstruppen begaben sich Teile der für die Besatzungsverwaltung in Deutschland vorgesehenen Offiziere im August 1944 von England nach

24 Nach dem ${ }_{n}$ Chronicle of the Office of Military Government for Germany (U.S.) and its predecessor organizations" (Entwurf der Historical Section, OMGUS, Juni 1948) [zit.: Chronicle], wurde am 16./17.2.1944 die German Country Section, später German Country Unit begründet (3/148-3); Latour/Vogelsang: Okkupation und Wiederaufbau, S. 33; Steger: Clays Stabskonferenzen, S.114.

${ }^{25} \mathrm{Vgl}$. Chronicle.

${ }^{26}$ Latour/Vogelsang: Okkupation und Wiederaufbau, S. 28ff. Vgl. USGCC 1944-45/3/1; AG 1945-46/12/10.

${ }^{27}$ Chronicle. Vgl. Clay: Entscheidung in Deutschland, S. 22; Ziemke: The U.S. Army, S. 83 ff. 
Frankreich, im wesentlichen als Angehörige von G-5, SHAEF. Im Herbst waren alle Detachments, die für eine Besatzung vorgesehen waren und nicht aus besonderen Gründen in London zurückgehalten wurden, in die Nähe von Paris verlegt worden. Die German Country Unit als solche blieb dagegen in London bestehen und stellte in gewissem Sinne eine amerikanisch-britische Personalreserve für die Beschickung eines künftigen alliierten Kontrollorgans für Deutschland dar. ${ }^{28}$ So entwickelte man gegenüber den Sowjets Vorschläge für eine künftige gemeinsame Besatzungsverwaltung, doch kam es nicht zu einer Einigung der drei Mächte.

\section{Die Tätigkeit der Militärregierung während der Eroberung Deutschlands}

Während der letzten Wochen ihres Bestehens hatte die German Country Unit den Auftrag, detaillierte Pläne für die Aufstellung von besonderen Einheiten für die Military Government Detachments auszuarbeiten, wobei der deutsche Verwaltungsaufbau und die besonderen Aufgaben der Militärregierung auf den verschiedenen Ebenen zu berücksichtigen waren. Größe und Zusammensetzung eines Detachment waren von seinem Auftrag und dem Umfang des Gebietes, das es verwalten sollte, abhängig. Die Civil Affairs Detachments in den befreiten Ländern hatte man in absteigender Reihenfolge in vier Typen eingeteilt: A, B, C und D. In Deutschland, das nicht als befreites Land galt, hatte man fünf Klassen mit den Bezeichnungen E, F, G, H und I unterschieden. ${ }^{29}$

Die E-Detachments waren für Verwaltungsaufgaben in den größeren politischen Einheiten wie früheren preußischen Provinzen, Ländern oder besonders großen Stadtkreisen vorgesehen. Sie standen in der Regel unter dem Befehl eines Oberst und unterschieden sich je nach Einsatzort deutlich in der Aufgabenverteilung. Die Arbeitsgebiete, für die Spezialoffiziere zur Verfügung standen, reichten vom Hafenwesen über Erdölraffinierung bis zum Fischereiwesen. Im Normalfall waren folgende Bereiche vertreten: Regierung und Verwaltung, Rechtswesen und Gerichte, öffentliche Sicherheit, Gesundheitswesen, Handel und Industrie, Verkehr und Transport, Ernährung, Landwirtschaft, Finanzwesen, Banken und Versicherungen, öffentliche Arbeiten, Versorgungsbetriebe, Erziehungswesen, Kirchenangelegenheiten, Archivwesen, Kunst, Denkmalschutz und Nachrichtendienst.

Die F-Detachments waren für die Übernahme größerer Regierungsbezirke und Stadtkreise vorgesehen. Die G-Detachments waren erheblich kleiner und hatten die Aufgabe, die übrigen Regierungsbezirke, mittelgroße Stadtkreise und größere Landkreise zu betreuen. Im allgemeinen verfügten sie lediglich über Fachleute für öffentliche Sicherheit, Gesundheitswesen, Handel und Industrie, Finanz- und Rechtswesen. Die $\mathrm{H}$ - und I-Detachments waren damit betraut, Landkreise mittleren Umfangs und kleine Stadtkreise zu überwachen. Sie verfügten nur etwa über ein halbes Dutzend Offiziere und vielleicht zehn Soldaten der Mannschaftsdienstgrade. Die I-Detachments unterschieden sich von den $\mathrm{H}$-Detachments nur dadurch, daß sie kleinste oder dünn besiedelte Kreise übernehmen sollten.

${ }^{28}$ Chronicle; Ziemke: The U.S. Army, S.82f.; Steger: Clays Stabskonferenzen, S.114. Vgl. USGCC 1944-45/3/6.

${ }^{29}$ Latour/Vogelsang: Okkupation und Wiederaufbau, S. $37 \mathrm{ff}$. 
Für militärische wie administrative Zwecke wurden jeweils mehrere Detachments unterschiedlichen Typs zu einer Militärregierungs-Kompanie zusammengestellt. Diese konnten einer kämpfenden Einheit entweder zugeteilt oder beigestellt werden, solange der Krieg auf deutschem Boden andauerte.

Die militärische Eroberung großer Teile Deutschlands durch die amerikanische Armee vollzog sich von September 1944 bis Mai 1945. ${ }^{30}$ Sie begann Mitte September 1944 an der luxemburgischen Grenze; im dünn besiedelten Dreiländereck Deutschland-Belgien-Luxemburg lieB sich eine der ersten Einheiten der Militärregierung in Deutschland, das Detachment I8G2, im Dorf Winterscheid nieder. ${ }^{31}$ Weitere Abteilungen folgten. Während die 1. und 3. amerikanische Armee Teile des Rheinlands eroberten, gelang es der 7. Armee, die gegen das Saarland und die Pfalz operierte, erst Mitte Dezember, nach Deutschland vorzustoßen. Gegen Ende des Jahres 1944 umfaßte der von amerikanischen Streitkräften besetzte Teil Deutschlands lediglich Randgebiete entlang der belgischen, luxemburgischen und französischen Grenze. ${ }^{32}$ Obwohl dieser Raum nur über eine größere Stadt verfügte (Aachen), sollte er das „Versuchsgelände für die amerikanische Militärregierung“ in Deutschland werden.

Praktisch spielte sich die Übernahme der Regierungsgewalt auf regionaler Ebene meist so ab, daß nach der Beschlagnahmung geeigneter Räume als Quartiere und Büros für den Bedarf der Amerikaner geeignete deutsche Zivilisten als Bedienstete und Dolmetscher in den Kommunalverwaltungen ${ }^{33}$ eingesetzt wurden, wobei die Offiziere gehalten waren, sich über die von ihnen ausgewählten Kandidaten mit dem örtlichen Klerus, den Richtern und „anderen prominenten Persönlichkeiten“ zu besprechen. ${ }^{34}$

General Eisenhower als Oberkommandierender der alliierten Streitkräfte erlieB im September 1944 die Proklamation Nr. 1 an die deutsche Bevölkerung. ${ }^{35}$ Danach kamen die alliierten Streitkrafte als ",siegreiches Heer, jedoch nicht als Unterdrucker“. Die Vernichtung des Nationalsozialismus und des deutschen Militarismus wurde angekündigt. Führer der Wehrmacht und der NSDAP, Mitglieder der Geheimen Staatspolizei und andere schuldige Personen sollten gerichtlich angeklagt und gerecht bestraft werden. Die Politik der amerikanischen Militärregierung wollte sich damals noch auf den bestehenden deutschen Verwaltungsapparat stutzen. Daher hatte auch Eisenhowers Proklamation Nr. 1 den Satz enthalten: „Alle Beamten sind verpflichtet, bis auf weiteres auf ihrem Posten zu verbleiben.“ Dieser Befehl erwies sich jedoch als nicht durchführbar, da der größere Teil der Bürgermeister und leitenden Kommunalbeamten sich mit den deutschen Truppen abgesetzt hatte oder untergetaucht war. ${ }^{36}$ Die Auswahl und Ernennung neuer Bürgermeister oder anderer Beamter erfolgten nach keinem strengen Plan und waren häufig Zufälligkeiten unterworfen. Nach Einrichtung der Gemeindeverwaltungen bemüte man sich auch um entsprechende Verwaltungen auf Kreis- und Bezirksebene. Da die deutschen Gerichte auf Befehl der Militärregierung geschlossen worden waren, hatte die Military Government Ordinance Nr. 2 die Bildung von Militärregierungsgerichten für Verfahren gegen Deutsche und

\footnotetext{
30 Ziemke: The U.S. Army, S. $133 \mathrm{ff}$.

31 Latour/Vogelsang: Okkupation und Wiederaufbau, S. $40 \mathrm{ff}$.

32 Vgl. USGCC 1944-45/1/1.

33 Vgl. beispielhaft Güstrow: In jenen Jahren, S. $36 \mathrm{ff}$.

34 Latour/Vogelsang: Okkupation und Wiederaufbau, S.45 f.

35 Amtsblatt der Militärregierung Deutschland. Amerikanisches Kontrollgebiet, S.1.

36 Latour/Vogelsang: Okkupation und Wiederaufbau, S. 45.
} 
andere in Deutschland lebende Zivilpersonen angeordnet. ${ }^{37} \mathrm{Zu}$ den Hauptaufgaben der deutschen Verwaltung gehörte es, die Bevölkerung zu veranlassen, die Verordnungen der Militärregierung zu befolgen.

Der entscheidende Vorsto $B$ der westlichen Allierten in das Innere Deutschlands begann am 8. Februar 1945; am 7. März wurde der Rhein bei Remagen überquert. ${ }^{38}$ Von diesem Zeitpunkt an war klar, daB die Amerikaner nicht nur das ihnen ursprünglich zugeteilte süddeutsche Gebiet besetzen, sondern zugleich auch für etwa die Hälfte der britischen und ein Fünftel der späteren sowjetischen Besatzungszone vorübergehend verantwortlich sein würden. Der Aufbau einer neuen deutschen Verwaltung auf der untersten Ebene gestaltete sich auch in diesen Gegenden überaus mühselig; ${ }^{39}$ als noch schwieriger erwies sich die Aufgabe in den Stadt- und Landkreisen. Dabei zeigte es sich, daB die Militärregierung z. B. in Thüringen vor allem entlang der Hauptverkehrsader (Eisenach, Gotha, Erfurt, Weimar, Jena, Apolda, Gera) Detachments einsetzte, während es nicht gelang, abseits gelegene kleinere Städte und Dörfer von Anfang an unter wirksame Kontrolle der Militärregierung zu stellen.

Erste Bemühungen um die Verwaltung ganzer Provinzen oder Länder setzten naturgemäß im Westen des Reiches ein. ${ }^{40}$ Nach der Kapitulation ging man sofort daran, das Gebiet der 15. Armee verwaltungsmäBig auf höherer Ebene zu organisieren. In Neustadt an der Weinstraße wurde am 10. Mai 1945 das Oberpräsidium Saarland-PfalzSüdhessen ins Leben gerufen, ${ }^{41}$ das als Teil des Rhein Province Military District vorgesehen war. Dieses und ähnliche Gebilde waren jedoch nur sehr kurzlebig, da die Engländer und die Franzosen am 21.Juni bzw. 10. Juli 1945 ihre Besatzungszonen in den endgültigen Grenzen übernahmen. ${ }^{42}$

Die amerikanische Zone umfaßte nunmehr das (rechtsrheinische) Bayern (ohne den Landkreis Lindau), Württemberg-Baden (die nördlichen Teile der alten Länder Württemberg und Baden), Groß-Hessen (ohne die an die französische Zone gefallenen nassauischen und rheinhessischen Gebietsteile) sowie - mit sachlichen und zeitlichen Einschränkungen - die Enklave Bremen. Der amerikanische Sektor von Berlin gehörte formell nicht zur amerikanischen Zone.

Mit Wirkung vom 1. August 1945 wurde das amerikanische Besatzungsgebiet in zwei Militärdistrikte eingeteilt. Der östliche (Eastern Military District) unter General George S. Patton umfaßte Bayern (ohne den Landkreis Lindau), der westliche (Western Military District) ${ }^{43}$ unter General Wade H. Haislip Württemberg-Baden und Hessen. Die politische Koordinierung lag im Osten bei der 3. Armee mit Hauptquartier in Bad Tölz, im Westen bei der 7. Armee mit Hauptquartier in Heidelberg. Bremen hatte einen besonderen Status; der Kommandierende General im amerikanischen Sektor von Berlin war Lucius D. Clay unmittelbar unterstellt.

\footnotetext{
37 Amtsblatt der Militärregierung Deutschland - Amerikanisches Kontrollgebiet, S. 60 ff.

38 Ziemke: The U.S. Army, S. 184 ff.; Latour/Vogelsang: S. $62 \mathrm{ff}$.

39 Güstrow: In jenen Jahren, S.46ff.

${ }^{40}$ Latour/Vogelsang: Okkupation und Wiederaufbau, S.66ff.

41 Quellen zur Geschichte von Rheinland-Pfalz, S.21, Anm. 1.

42 Ziemke: The U.S. Army, S. $308 \mathrm{ff}$.

$43 \mathrm{Vgl}$. USGCC 1944-45/12/6; USGCC 1944-45/68/7; FA 68/8.
} 


\section{Die US Group Control Council als Vorläufer von OMGUS ${ }^{44}$}

Nicht zuletzt, um jeden Anschein eines britisch-amerikanischen Einverständnisses gegen die Sowjetunion in der Europäischen Beratenden Kommission, die sich wegen des sowjetischen Desinteresses nicht auf eine Tripartite Control Machinery einigen konnte, zu vermeiden, beschlossen die britische und die amerikanische Führung, die Pläne für eine gemeinsame Militärregierung aufzugeben und statt dessen eine amerikanische und eine britische Gruppe für die Planung und Ausführung der Politik künftiger Militärregierungen auf rein nationaler Grundlage zu konstituieren. ${ }^{45}$ Ende Juni 1944 schickte General Eisenhower Brigadegeneral Cornelius W. Wickersham nach Washington, um die Genehmigung des War Department für die Errichtung eines Planungsstabes zur Vorbereitung der Verwaltung in Deutschland zu erhalten. Die formelle Genehmigung des Vereinigten Generalstabs traf am 5. August 1944 bei General Eisenhower ein. Durch General Order Nr.80, ETO, vom 9. August 1944 wurde die US Group Control Council (USGCC) (Germany) in der Nähe von London errichtet. ${ }^{46}$ Sie wurde Wickersham in dessen Eigenschaft als Stellvertreter des noch nicht ernannten Repräsentanten der Vereinigten Staaten beim Kontrollrat unterstellt. ${ }^{47}$ Als ehemaliger Direktor der Militärregierungsschule an der Universität von Virginia und - seit dem 21. Mai 1944 - Deputy Chief of the European Allied Contact Section von SHAEF hatte er ein umfassendes Konzept für die Organisation und den Personaleinsatz von USGCC entwickelt. Die USGCC war formell ETOUSA unterstellt, gehörte jedoch für die Dauer der Doppelfunktion Eisenhowers in Deutschland zum Bereich von SHAEF, um im Auftrag der amerikanischen und der britischen Regierung die Politik durchführen zu können, auf die sich alle drei alliierten Mächte geeinigt haben würden.

Am Anfang bestand die USGCC aus drei (Haupt-)Abteilungen. Die Armed Forces Division $^{48}$ unter General John E. Lewis hatte sich um die Entwaffnung der Wehrmacht, das künftige Schicksal der deutschen Soldaten, die Entmilitarisierung Deutschlands, um die Betreuung alliierter Kriegsgefangener in Deutschland und deren Rückkehr in die Heimat zu kümmmern sowie nicht zuletzt nachrichtendienstliche Aufgaben auch unter Verwendung deutscher Wissenschaftler und Forscher wahrzunehmen.

Die übrigen - auch nicht im weiteren Sinne des Wortes militärischen - Aufgaben hatten die beiden anderen (Haupt-)Abteilungen zu übernehmen, die recht willkürlich gebildet wurden. Die Military Government Division A unter General Eric F. Wood beschäftigte sich mit allen Fragen der Wirtschaftsplanung, zu denen man auch die Bereiche Finanzen, Recht, Vermögen, Verkehr und Nachrichtenwesen rechnete. Division B unter Brigadegeneral Bryan L. Milburn war die eigentlich politische Abteilung, die sich auch mit Angelegenheiten der öffentlichen Sicherheit, der Wohlfahrtspflege, der Displaced Persons, dem Bereich Arbeit und Sozialwesen sowie mit der Verwal-

44 Ziemke: The U.S. Army, S. 94 ff. Ein Kriegstagebuch von USGCC ist überliefert in AG 194546/59/2. Vgl. außerdem USGCC 1944-45/1/3; USGCC 1944-45/3/4; USGCC 1944-45/12/4; USGCC 1944-45/13/7, USGCC 1944-45/13/10; USGCC 1944-45/14/1; USGCC 1944-45/19/5; USGCC 1944-45/20/1-7; AG 1945-46/46/7; AG 1945-46/58/5-6; AG 1945-46/59/1-2; AG 1945-46/61/7; AG 1945-46/62/2-3;5/11-1/1-5; 5/361-2/19.

45 Steger: Clays Stabskonferenzen, S. 114, Anm. 8.

46 Chronicle und andere Unterlagen in 3/148-3.

47 Steger: Clays Stabskonferenzen, S. 115f.; Ziemke: The U.S. Army, S. 93.

48 Steger: Clays Stabskonferenzen, S.116. Vgl. USGCC 1944-45/10/7. 
tung Berlins zu beschäftigen hatte. Eine Gliederung in Unterabteilungen war vorgesehen, kam jedoch nur teilweise zustande.

Amerikanische Experten haben an dieser Organisation deutliche Kritik geübt. ${ }^{49}$ Der Verwaltungsaufbau sei in der operativen Phase der Militärregierung viel zu groß und schwerfällig gewesen. Man habe den Eindruck, daß Neigungen von Experten im Lehnstuhl in Washington größeren Einfluß gehabt hätten als die Erfahrungen von Offizieren an der Front. So sei zwar die Einteilung der Armed Forces Division in drei Unterabteilungen (Heer, Marine, Luftwaffe) vernünftig gewesen, doch bleibe es unverständlich. warum in den beiden anderen Hauptabteilungen keine Unterabteilung für eine ganz wesentliche Aufgabe, nämlich die Entwicklung von Vorschlägen für die künftige deutsche Regierungs- und Verwaltungsstruktur, gebildet worden sei.

Grundsätzlich hatte USGCC eine doppelte Aufgabe zu erfüllen. Sie war. solange der Krieg noch andauerte, nicht nur amerikanische Planungsstelle für Deutschland, sondern sollte auch nach dem Ende der Feindseligkeiten die Rolle eines obersten amerikanischen Hauptquartiers für Fragen der Militärregierung übernehmen. Es lag nahe, die USGCC so zu organisieren, daß ihr Aufbau deutscher Verwaltungsstruktur entsprochen hätte. Dies wurde von amerikanischer Seite im Grundsatz auch angestrebt. Die Rücksichtnahme auf die vorgesehene fachliche Gliederung der Dreimächte-Kontrollverwaltung, auf die sich die Alliierten am 14. November 1944 in der Europäischen Beratenden Kommission geeinigt hatten, führte jedoch zu der Konsequenz, daß die drei (Haupt-)Abteilungen von USGCC am 25. November 1944 durch General Order Nr. 3, USGCC, aufgelöst wurden und folgende zwölf (Fach-)Abteilungen (Divisions) an ihre Stelle traten:50

Ubersicht 1: Organisation und Personal von USGCC, November 1944

- Militärwesen (Army/Ground) unter Brig.Gen. John E. Lewis

- Marine (Naval) unter V.Adm. Robert L. Ghormley

- Luftwaffe (Air) unter Maj.Gen. Robert W. Harper

- Verkehr (Transport) unter Col. John A. Appleton

- Politik (Political) unter Amb. Robert D. Murphy

- Wirtschaft (Economics) unter Col. Graham K. Howard

- Finanzen (Finance) unter Col. Bernard Bernstein

- Reparationen, Lieferungen und Restitutionen (Reparations, Deliveries and Restitutions) unter Lt.Col. C. S. Reid

- Innere Angelegenheiten und Nachrichtenwesen (Internal Affairs and Communications) unter Brig.Gen. Frank C. Meade

- Justiz (Legal) unter Lt.Col. J. B. Marsh

- Kriegsgefangene und Verschleppte (Prisoners of War and Displaced Persons) unter Brig.Gen. Eric F. Wood

- Arbeit (Manpower) unter Maj. David A. Morse

An der Spitze der Divisions standen Directors; Brigadegeneral Bryan L. Milburn wurde zum Chefkoordinator für Planung ernannt. ${ }^{51}$ Eine besondere Einheit für nachrichtendienstliche Aufgaben (Intelligence Section) trat hinzu, die gleichzeitig für Fragen der Zensur (ohne Pressezensur) verantwortlich war. An ihrer Spitze stand Oberst Charles $\mathrm{C}$. Blakeney. Gleichzeitig rief man einen Koordinierungsausschuß für nach-

\footnotetext{
${ }^{49}$ Zink: The United States in Germany, S. 27.

${ }^{50}$ Kurzbiographien Milburns und einiger Abteilungsleiter in Chronicle.

51 Vgl. General Order Nr.3, USGCC, vom 25.11.1944.
} 
richtendienstliche Aufgaben (Joint Intelligence Committee) ins Leben, in dem unter dem Vorsitz von Blakeney die Leiter der für nachrichtendienstliche Angelegenheiten verantwortlichen Organisationseinheiten in den Abteilungen für Militärwesen, für Marine, für Luftwaffe, für Politik sowie für Wirtschaft und für Finanzen zusammenge$\mathrm{faßt}$ waren. Der Sinn dieser neuen Organisation lag offensichtlich in der Entlastung des Kommandierenden Generals und in der Dezentralisierung der Aufgabenerledigung. Am 31. Oktober 1944 waren 145 Heeresoffiziere, je 15 Luftwaffen- und Marineoffiziere sowie ebenfalls 15 Zivilbedienstete bei USGCC eingesetzt. ${ }^{52}$ Eine Personalvermehrung erschien dringend erforderlich, bevor die USGCC London verlie $B$ und auf den Kontinent übersetzte. Gegen Ende 1944 wurden insgesamt 1200 Bedienstete als notwendig anerkannt. Die ursprünglich vor allem im Verhältnis zur German Country Unit recht schwierige Stellung von USGCC hatte sich durch die Vereinbarung, die am 14. November 1944 innerhalb der Europäischen Beratenden Kommission über den Kontrollapparat in Deutschland erzielt worden war, ${ }^{53}$ deutlich verbessert. Die German Country Unit hatte sich nämlich im August 1944 damit einverstanden erklärt, daß USGCC nach der Einsetzung der interalliierten Kontrollbehörde auch die Durchführung der Besatzungspolitik übernehmen sollte. Als Planungsstab für die in Aussicht genommene interalliierte Kontrollbehörde mußte sich USGCC an den Beschlüssen der Europäischen Beratenden Kommission orientieren und ihre Arbeit mit den britischen und sowjetischen Kontrollratsgruppen abstimmen. Die Verbindung zur britischen Control Commission (Military Section) (CCMS) durfte aber nur über die Kommandokanäle von G-5, SHAEF, erfolgen, damit sich kein Militärregierungsapparat außerhalb des üblichen Dienstweges entwickeln konnte. Nicht zuletzt hatte sich die USGCC aber auch nach den allgemeinen politischen Richtlinien des Kriegsministeriums in Washington, insbesondere der Civil Affairs Division, zu richten.

Wie schwer es war, unter solchen Bedingungen „längerfristige politische Konzeptionen" zu entwerfen, zeigt die Diskussion um die Direktive JCS 1067.54 Dieses Dokument war eine Anweisung des Vereinigten Generalstabs der amerikanischen Streitkräfte an Eisenhower in dessen Eigenschaft als Militärgouverneur in Deutschland. Nach der Fassung vom 22. September 1944 sollte der Militärgouverneur alle Inhaber von Schlüsselpositionen im politischen, wirtschaftlichen und öffentlichen Leben sowie alle nationalsozialistischen Funktionäre und alle Sympathisanten des NS-Regimes sofort verhaften. Demgegenüber verlangte aber die Vereinbarung über den Kontrollrat in Deutschland, daß die Abteilungen der Besatzungsverwaltung durch die ihnen sachlich korrespondierenden deutschen zentralen Behörden tätig werden sollten. Solche „Widersprüche, die Unsicherheit und die ständigen Revisionen in der amerikanischen Deutschlandplanung“" vor allem aber die Tatsache, daß USGCC innerhalb des militärischen Apparats als ein ,akademischer“ Planungsstab ohne Entscheidungsbefugnis und aktive Einsatzmöglichkeiten arbeiten mußte, ließen sie zu einem „kopflastigen Gebilde" werden, das sich aus Washington den Vorwurf der "Ineffektivität und Nutzlosigkeit" gefallen lassen mußte. ${ }^{55}$

Die räumliche Trennung der USGCC von SHAEF und ETOUSA brachte weitere Reibungsflächen. ${ }^{56}$ Am 15.Januar 1945 verdeutlichte ETOUSA, daß USGCC die

52 Chronicle, S. 18.

53 Graml: Die deutsche Frage, S. $282 \mathrm{f}$.

54 Deutsche Übersetzung in: Neubeginn und Restauration, S. $58 \mathrm{ff}$.

${ }^{55}$ Murphy: Diplomat unter Kriegern, S. 395; Steger: Clays Stabskonferenzen, S. 119; Ziemke: The US Army, S. $222 \mathrm{f}$.

$56 \mathrm{Vgl}$. Zink: The United States in Germany, S. 28. 
Ubersicht 2: Organisation von USGCC. November 1944

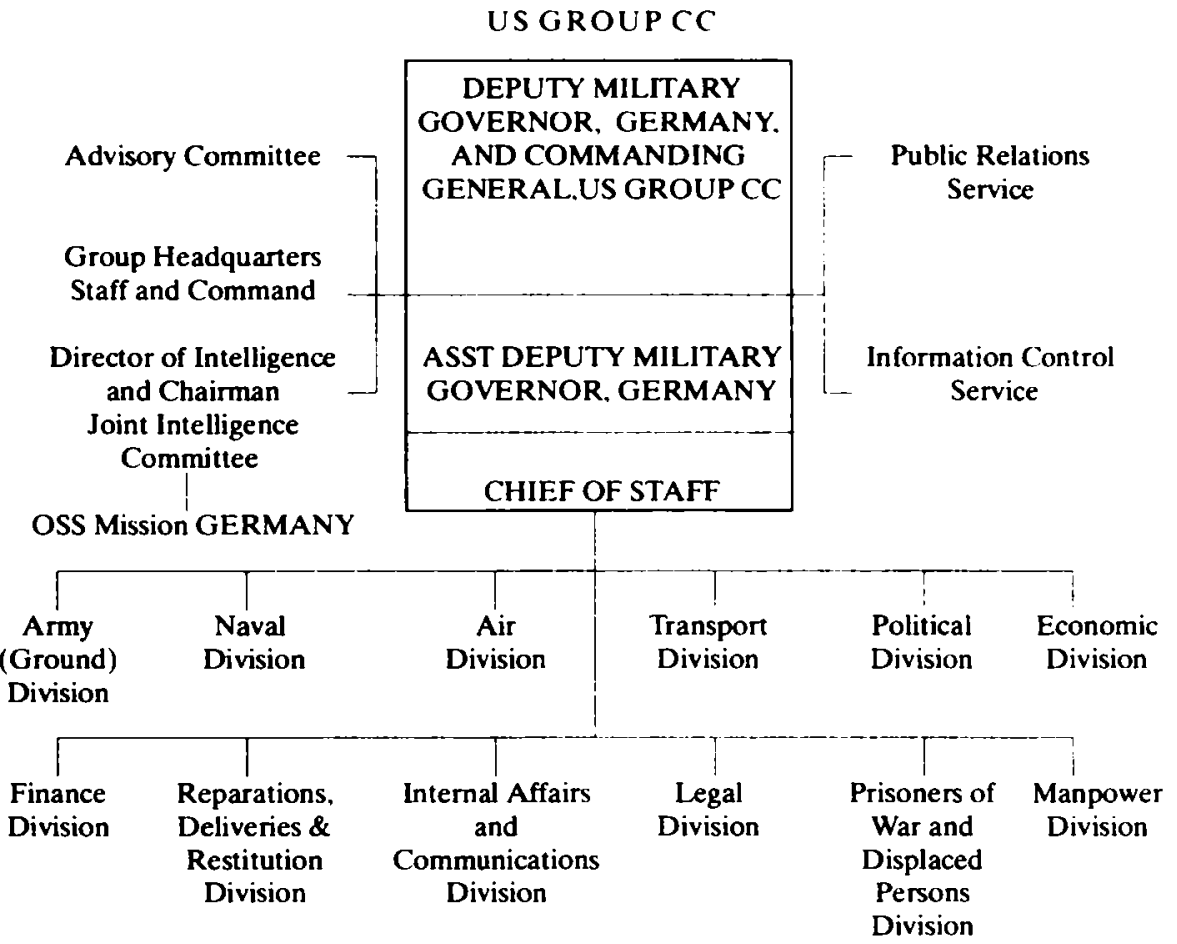

Aufgabe habe, für Eisenhower als Commanding General. ETO, umfangreiche Aufgaben bei der Vorbereitung der Besetzung Deutschlands zu erfüllen und als Eisenhowers Verbindungsstelle zur amerikanischen Delegation bei der Europäischen Beratenden Kommission in London zu fungieren. Die künftigen Kontakte der Vereinigten Staaten mit den anderen Alliierten sollten auf zwei Ebenen erfolgen: mit Sowjets und Briten im Rahmen des Dreimächte-Kontrollorgans und nur mit den Briten in ausschließlich SHAEF berührenden Fragen. Um die Arbeiten von USGCC und SHAEF besser koordinieren zu können, schlug General Wickersham vor, daß Teile des Personals von USGCC nach Versailles verlegt werden sollten. Zu Beginn des Jahres 1945 machte eine Vorhut (Advanced Section) den Anfang; ${ }^{57}$ am 18. April 1945 wurde die Dienststelle als solche in Versailles errichtet.

Als der deutsche militärische Widerstand gegen die vordringenden Truppen der Alliierten immer schwächer wurde, fiel im Frühjahr 1945 die Entscheidung über die Zukunft der USGCC im Zusammenhang mit der Berufung von Lucius D. Clay zum Stellvertretenden Militärgouvemeur (Deputy Military Govemor). ${ }^{58}$ Eisenhower hatte schon frühzeitig sein Desinteresse an der Zivilverwaltung in der amerikanischen Zone erkennen lassen, um die Hände für notwendige militärische Operationen frei

57 General Order Nr.1, USGCC, vom 27.1.1945 und General Order Nr.5, USGCC, vom 19.3.1945.

58 Steger: Clays Stabskonferenzen, S. 119. 
zu haben. Bevor Clay sein Amt antrat, hatte er Eisenhower seine Vorstellungen über seinen künftigen Aufgabenbereich erläutert. Er verlangte eine vom Armeekommando unabhängige und Eisenhower unmittelbar unterstellte Position als ...Adviser to the Chief of Staff and the Commanding General for Military Government within the US Zone of occupation". Als solcher wollte er unmittelbar durch G-5. SHAEF, mit den ihr untergeordneten Einheiten zusammenarbeiten. Eisenhowers Direktive über die Beziehungen zwischen der USGCC und G-5, SHAEF, vom 29. April 1945 entsprach diesen Forderungen nahezu bis in die letzten Einzelheiten. Clay wurde Deputy Military Governor und gleichzeitig Chief of Staff und Commanding General, USGCC. Durch die Verbindung dieser drei Ämter war sichergestellt, daß die Entwicklung von politischen Richtlinien und deren Anwendung in einer Hand lagen. Eisenhower seinerseits fungierte seit Ende April als Commanding General der amerikanischen Streitkräfte in Europa und gleichzeitig als Oberkommandierender der amerikanischen Zone Deutschlands. Er sollte außerdem der Vertreter der Vereinigten Staaten von Amerika in dem vorgesehenen Dreimächte-Kontrollorgan sein.

Die Ernennung Clays wurde am 31. März 1945 verkündet ${ }^{59}$ Bevor er Washington verließ, waren ihm nach seiner Darstellung die politischen Hintergründe für seinen Auftrag weitgehend unbekannt. Dies gilt auch für die bereits erwähnte Direktive JCS 1067, die Clay wegen ihrer Deutschland gegenüber ebenso rigorosen wie unvernünftigen Forderungen regelrecht schockierte, ohne daß eine offizielle AuBerkraftsetzung vor Mitte 1947 möglich gewesen wäre. ${ }^{60}$ Clay meldete sich am 8 . April bei Eisenhower in dessen Hauptquartier in Reims und wirkte erfolgreich darauf hin, nach Beendigung des Krieges die Aufgaben der künftigen Militärregierung vom „eigentlichen militärischen Befehlsbereich“" zu trennen. Damit war eine weitere entscheidende Weiche für die künftige Entwicklung der amerikanischen Militärregierung in Deutschland gestellt. Am 25. April 1945 übernahm Clay aufgrund der ihm durch General Order Nr. 75, ETOUSA, vom 23. April 1945 erteilten Ermächtigung formell das Kommando über USGCC. ${ }^{61}$

\section{Von der Kapitulation bis zur Reform von USGCC}

Als Deutschland am 8./9. Mai 1945 bedingungslos kapitulierte, waren sich die Alliierten noch nicht darüber einig, ob und wie sie Deutschland teilen wollten. Jedenfalls war im Text der Kapitulationsurkunde, der im Hauptquartier des Alliierten Oberbefehlshabers General Eisenhower eilends gefertigt worden war, von einer Aufteilung Deutschlands nicht die Rede. Im Auftrag des von Hitler als Reichspräsident eingesetzten Großadmirals Karl Dönitz bildete Reichsfinanzminister Lutz Graf Schwerin von Krosigk eine geschäftsführende Reichsregierung in Flensburg. Er versuchte, in Verhandlungen mit den Alliierten Voraussetzungen für die Einsetzung einer neuen deutschen Verwaltung zu schaffen. Diese geschäftsführende Regierung wurde jedoch am 23. Mai 1945 durch die Alliierten abgesetzt und verhaftet. Damit war das Deutsche Reich endgültig handlungsunfähig geworden.

${ }^{59}$ Clay: Entscheidung in Deutschland, S. $18 \mathrm{ff}$.

${ }^{60}$ Gimbel: Amerikanische Besatzungspolitik, S.16ff.

${ }^{61}$ General Order Nr. 18, USGCC, vom 25.4.1945. 


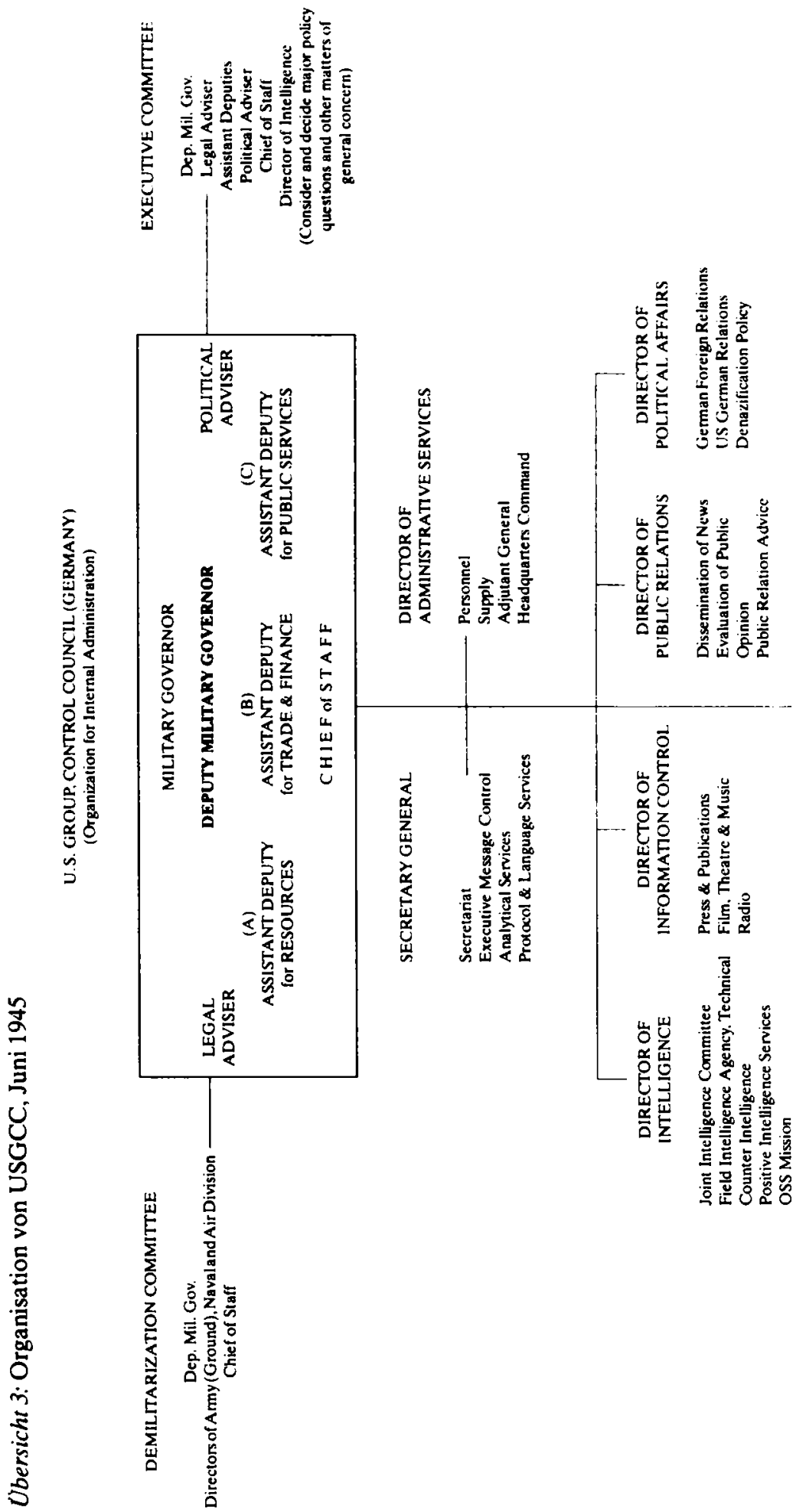




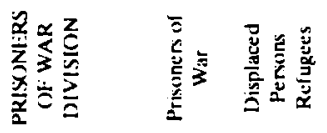

-1 -

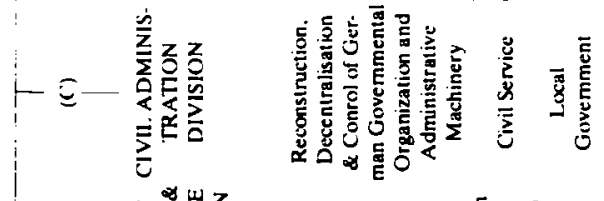

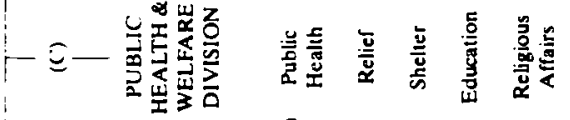

-

-

-

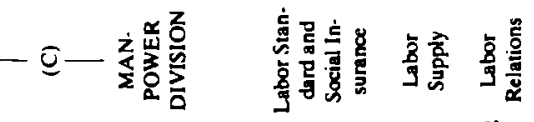

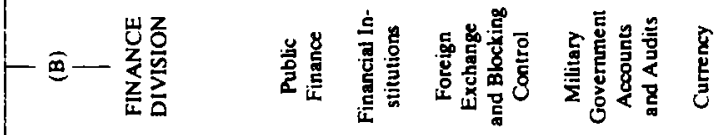

-

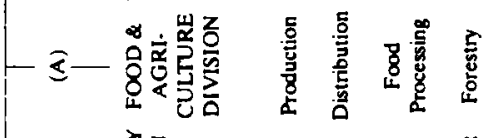

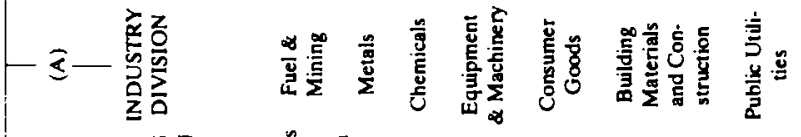

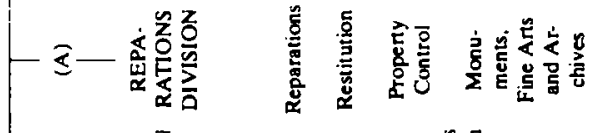

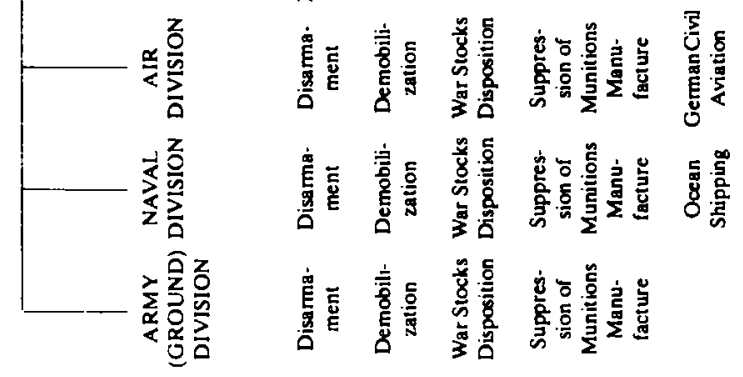


Im Unterschied zu den Vereinigten Staaten, Großbritannien und wohl auch der Sowjetunion hat Frankreich, das an den alliierten Kriegskonferenzen nicht beteiligt war. kontinuierlich das Ziel verfolgt. eine neue staatliche Zentralgewalt in Deutschland auch in Zukunft unter keinen Umständen entstehen zu lassen. ${ }^{62}$

Am 5.Juni 1945 unterzeichneten in Berlin die Militärbefehlshaber eine Erklärung ,.in Anbetracht der Niederlage Deutschlands und der Übernahme der obersten Regierungsgewalt" sowie drei ergänzende Vereinbarungen über das Kontrollverfahren, die Besatzungszonen und das Verhältnis Deutschlands zu den Mitgliedstaaten der zu gründenden Vereinten Nationen. Die Sieger erklärten, die gesamte öffentliche Gewalt in Deutschland von der zentralen Ebene bis hin zu den Gemeinden zu übernehmen, ohne damit Deutschland annektieren zu wollen. ${ }^{63}$ Diese Erklärung wurde auf $\mathrm{Be}$ schluß des Kontrollrats dem deutschen Volk am 30. August 1945 in Form einer Proklamation bekannt gegeben. ${ }^{\text {th }}$

Am Tage der bedingungslosen Kapitulation wurden Teile von USGCC von Versailles nach Frankfurt-Höchst verlegt und im I.G. Farben-Gebäude untergebracht, zumal noch nicht sicher war, ob Berlin Sitz eines künftigen alliierten Kontrollorgans sein würde. Erst nach der entsprechenden Einigung der vier Alliierten vom 5. Juni 1945 setzte sich ein amerikanisches Vorauskommando nach Berlin in Bewegung. Am 14. Juni wurde die USGCC-Dienststelle in Versailles geschlossen. ${ }^{65}$ Der Umzug von Frankfurt-Höchst nach Berlin war am 3. August 1945 beendet. ${ }^{66}$

Ubersicht 4: Organisation und Personal von USGCC, Juni 1945

\section{Ofince of the Deputy Military Govemor}

Deputy Military Governor

Special Assistants to the Deputy

Military Governor

Assistant Deputy Military Governor

Chief of Staff

Director of Administration

Analytical Section, Chief

Control and Records Section, Chief

Liaison and Records Section, Chief

Adjutant General

Personal Section, Chief

Intelligence Section. Dep. Chief

\section{Ammy Ground Division}

Director

Deputy Director

$\begin{array}{ll}\text { Lt.Gen. } & \text { Lucius D. Clay } \\ \text { Civ. } & \text { L.W. Douglas } \\ \text { Civ. } & \text { R. L. McCaffery } \\ \text { Civ. } & \text { L.S. Fish } \\ \text { Lt.Col. } & \text { R.R. Bowie } \\ \text { Lt.Col. } & \text { R.C.Sanders } \\ \text { Capt. } & \text { N.S. Amold } \\ \text { Capt. } & \text { F.T. Dunscomb } \\ \text { Maj.Gen. } & \text { Oliver P. Echols } \\ \text { Brig.Gen. } & \text { Bryan L. Milbum } \\ \text { Col. } & \text { T.W. Hammond } \\ \text { Col. } & \text { H.L.Carter } \\ \text { Lt.Col. } & \text { Charles E. Sands } \\ \text { Lt.Col. } & \text { O. Pantuhoff } \\ \text { Lt.Col. } & \text { James L. Williams } \\ \text { Col. } & \text { Robert H.Chard } \\ \text { Col. } & \text { Theodore J.Koenig }\end{array}$

Brig.Gen. John E. Lewis

Col. W.R. Frost

\footnotetext{
${ }^{62}$ Hurwitz: Eintracht der Siegermächte, S. $58 \mathrm{ff}$.

${ }^{63}$ Neubeginn und Restauration, S.85 ff. Vgl. USGCC 1944 45/1/3.

64 Amtsblatt des Kontrollrats in Deutschland, Ergänzungsblatt Nr. 1, S. 7 ff.

65 General Order Nr. 30 vom 14.6.1945.

66 General Order Nr.41 vom 30.7.1945. Ein Vorauskommando von USGCC wurde bereits am 5.7.1945 in Berlin errichtet (General Order Nr. 36 vom 3.7.1945).
} 
Demobilization Branch. Chief

OKW-OKH Control Branch, Chief

Disarmament Branch, Chief

Intelligence Branch, Chief

\section{Naval Division}

Director

Disarmament Branch, Chief

Demobilization Branch, Chief

Intelligence Branch, Chief

Ocean Shipping Branch, Chief

\section{Air Division \\ Director \\ Political Division \\ Director \\ Deputy Director}

Administrative Branch, Chief

Disarmament Branch, Chief

Demobilization Branch, Chief

Policy and Liaison Branch, Chief

Operations Branch, Chief

Intelligence Branch, Chief

\section{Economic Division}

Director

Deputy Director

\section{Internal Afiairs and Communications Division}

Director

Deputy Director

Assistant Director

Communications and Education Branch, Chief

Civil Communications Branch, Chief

Education and Religious Affairs Branch, Chief

Civil Service and Local Government Branch. Chief

Public Safety Branch Chief

Public Health and Welfare Branch Chief
Col.

Col.

Col.

Col.

Col.

Col.

Col.

Col.

Col.

Col.

Col.

Col.

Col.

Col.

Col.

Col.

Col.

$\begin{array}{ll}\text { V.Adm. } & \text { Robert L. Ghormley } \\ \text { Capt. } & \text { A.H.Graubert } \\ \text { Capt. } & \text { E.C. Kline } \\ \text { Capt. } & \text { W.A. Finn } \\ \text { Comdr. } & \text { D.J.Harkins }\end{array}$

F.M. Albrecht

C.H. Blumenfeld

F.E.Emery

P.S. Lauben

W.W. Reinburg

T.B. Whitted

G.J.Engelthaler

W. L. Kluss

R.E.Jones

R.O.Montgomery

D. A. Taylor

H.S.Strubie

J.R. Culleton

W. W. Holler

D.S. Roysdon

R.W. Yates

Charles C. Blakeney

$\begin{array}{ll}\text { Maj.Gen. } & \text { Robert W. Harper } \\ \text { Col. } & \text { J.H.Pool } \\ \text { Col. } & \text { H.F.Muenter } \\ \text { Col. } & \text { F.M.S. Miller } \\ \text { Col. } & \text { J.N. DeRussy } \\ \text { Col. } & \text { G.E.Sisco } \\ \text { Col. } & \text { C.A.Young } \\ & \\ \text { Amb. } & \text { Robert D.Murphy } \\ \text { Civ. } & \text { Donald R. Heath }\end{array}$

Brig.Gen. William H. Draper

Col. John R. Gilchrist

$\begin{array}{ll}\text { Maj.Gen. } & \text { Oliver P. Echols } \\ \text { Col. } & \text { David Marcus } \\ \text { Col. } & \text { S.R. Brentnall } \\ \text { Brig.Gen. } & \text { Frank C. Meade } \\ \text { Brig.Gen. } & \text { C.O. Bickelhaupt } \\ \text { Maj. } & \text { M.M. Knappen } \\ \text { Brig.Gen. } & \text { Luther S.Smith } \\ \text { Col. } & \text { R. Bonnevalle } \\ \text { Col. } & \text { Arthur H. Onthank } \\ \text { Col. } & \text { Orlando W. Wilson } \\ \text { Col. } & \text { Robert W.Chamberlin } \\ \text { Maj.Gen. } & \text { Morrison C.Stayer } \\ \text { Col. } & \text { H. Hansen }\end{array}$




\section{Finance Division}

Director

Public Finance Branch, Chief

Financial Institutions Branch, Chief

Foreign Exchange and Blocking Control Branch. Chief

Intelligence and Liaison Branch. Chief

Accounts and Audits Branch, Chief

Currency Branch, Chief

\section{Transportution Division}

Director

Deputy Director

Inland Waterways Branch, Chief

Movement and Traffic Branch, Chief

Planning Branch, Chief

Ports and Coastal Shipping Branch, Chief

Rail Branch, Chief

Road Branch, Chief

Prisoner of War and Displaced Persons Division

Director

Deputy Director

Displaced Persons Branch, Chief

Prisoner of War Branch. Chief

\section{Reparations, Deliveries and Restitution Division}

Director

Deputy Director

\section{Legel Division}

Director

Legal Advice Branch, Chief

Justice Ministry Branch, Chief

Judicial Branch, Chief

Prisons Branch, Chief

\section{Manpower Division}

Director

Deputy Director

Deputy Director

Labor Ministry Section, Chief

Labor Intelligence Section, Chief

Nazi Labor Institutions Section, Chief

Labor Standards and Social Insurance Branch, Chief

Labor Allocations Branch, Chief

Labor Relations Branch, Chief

Housing Branch, Chief

\section{Headquarters Command}

Office of the Headquarters Commandant

Commandant

$\begin{array}{ll}\text { Col. } & \text { Bemard Bernstein } \\ \text { Lt.Col. } & \text { M.P.Fisher } \\ \text { Lt.Col. } & \text { J.E.McDonougy } \\ \text { Civ. } & \text { O.A.Schmitt } \\ \text { Civ. } & \text { A. M. Kamarck } \\ \text { Col. } & \text { M.S.Hudtluff } \\ \text { Lt.Col. } & \text { H.D.Cragon }\end{array}$

Brig.Gen. John A. Appleton

Col. D.E. Brisbine

Lt.Col. H. A. Knoll

Lt.Col. E.B.McKinney

Lt.Col. H.H. Yokes

Comdr. F.H.Foster

Lt.Col. W. G. White

Lt.Col. J.L.S.Snead

Brig.Gen. Eric F. Wood

Col. W.H.E.Holmes

Col. H.W. Anderson

Lt.Col. C.H.Ott

Col. Leslie W. Jefferson

Col. H.C.Newton

$\begin{array}{ll}\text { Col. } & \text { J.B.Marsh } \\ \text { Col. } & \text { John M.Raymond } \\ \text { Col. } & \text { E. L. McLendon } \\ \text { Maj. } & \text { D.F. White } \\ \text { Col. } & \text { A.B.Michell }\end{array}$

Lt.Col. David A. Morse

Civ. J.Keenan

Lt.Col. R.B.Shuman

Lt.Col. O.S.Carpenter

2nd Lt. $\quad$ D. A. Courtney

Civ. George S. Wheeler

Civ. P.R.Porter

Lt.Col. A.H.Aronson

Civ. M.B.Wolf

Maj. D.Snyder

Col. $\quad$ F.R.Lafferty

Ende Juni 1945 wurde USGCC nach überaus intensiver interner Vorbereitung von einer Planungsgruppe in eine obere Behörde für die Militärregierung in Deutschland umgewandelt. ${ }^{67}$ ETOUSA wurde zur Kommandobehörde und - mit Sitz in Frankfurt

$675 / 376-1 / 27 ; 3 / 176-3 / 7$. 
am Main - am 1. Juli 1945 in US Forces European Theater (USFET) umbenannt, das ausschließlich amerikanische Truppen befehligte, allerdings nicht nur für die in der amerikanischen Zone. sondern auch für die in Großbritannien, Frankreich, Belgien, Norwegen und Österreich verantwortlich war. Zwei Wochen später, am 14. Juli 1945. wurde die Auflösung von SHAEF formal vollzogen. Die 12. amerikanische Heeresgruppe, die bisher Besatzungstruppen befehligt hatte, stellte ihre Tätigkeit am 27. Juli 1945 ein; die Truppen wurden USFET unmittelbar unterstellt. Im übrigen war der Machtkampf zwischen G-5, SHAEF, und USGCC Ende Juni zugunsten von Clay entschieden, der sich mit General Clarence L. Adcock, dem Leiter von G-5, SHAEF, darauf einigte, das Personal beider Stellen in Fachabteilungen zu vereinigen, die je nach Zweckmäßigkeit ihren Sitz in Berlin oder Frankfurt haben sollten. ${ }^{68}$

Da die Zuständigkeiten von G-5, SHAEF, der Natur der Sache nach auf G-5, USFET, übergegangen waren, bestanden weiterhin zwei Militärregierungsstäbe, die für die Bildung des amerikanischen Anteils (Element) in der Alliierten Kontrollbehörde und zur Herrschaft über die amerikanische Zone in Betracht kamen. ${ }^{69}$

Durch General Order Nr.33 vom 29. Juni 1945 ergab sich im einzelnen folgendes Bild der Organisation der Militärregierung (USGCC): $:^{70}$ Während Eisenhower bis zu seiner Ablösung durch General Joseph T. McNarney am 30. November 1945 formal die Funktion eines Militärgouverneurs behielt, wurde die Verwaltung von Lucius D. Clay als Stellvertretendem Militärgouverneur geleitet. Clay selbst stand an der Spitze von drei Verwaltungseinheiten innerhalb der Organisation: dem Executive Office (Office of the Deputy Military Governor), dem Executive Committee und dem Demilitarization Committee.

Zum Executive Office gehörten neben Clay als Stellvertretendem Militärgouverneur und Kommandierendem General, USGCC, ein Political Adviser, Botschafter Robert D. Murphy, ein Legal Adviser, Charles Fahy, ein Assistant Deputy for Public Services, Generalmajor Oliver P. Echols, ein Assistant Deputy for Resources, Brigadegeneral William H. Draper, ein Assistant Deputy for Trade and Finance, Laird Bell, und der Chief of Staff, Brigadegeneral Bryan L. Milburn. Während die Zuständigkeiten des Politischen und des Rechtsberaters offenkundig sind, waren die Assistant Deputies für politische Grundsatzfragen in jeweils drei Gruppen von Sachgebieten zuständig, die von den Fachabteilungen im einzelnen bearbeitet wurden. Ein Assistant Deputy for Political Affairs wurde nicht emannt, ${ }^{71}$ vielmehr die bisherige Political Division durch einen Director of Political Affairs ersetzt, da die „politische“ Funktion dieser Stelle eher beratender als operativer Natur war.

Neben diesem obersten Führungsorgan, dem Executive Office, bestanden zwei Ausschüsse: das Executive Committee und das Demilitarization Committee. Der erste Ausschuß umfaßte unter Leitung des Deputy Military Governor die drei Assistant Deputies, den Politischen und den Rechtsberater, den Chef des Stabes und den Direktor für nachrichtendienstliche Aufgaben (Director of Intelligence). Dem Demilitarization Committee, das ebenfalls vom Deputy Military Governor geleitet wurde, gehörten die Direktoren der Heeres-, Marine- und Luftwaffenabteilungen sowie der Chef des Stabes an.

68 Ziemke: The U.S. Army, S. 402.

${ }^{69}$ Steger: Clays Stabskonferenzen, S.119f. Vgl. USGCC 1944-45/12/5. USFET Military Government Weekly Field Reports liegen von August 1945 bis Januar 1946 in 5/367-3/11 vor.

70 Die personelle Zusammensetzung wurde durch General Order Nr. 34, USGCC, vom 29.6.1945 bekanntgegeben.

7 5/376-1/27. 
Als dritter Ausschuß bestand ein Joint Intelligence Committee, das allerdings nicht zur Spitzengliederung der Militärregierung gehörte und vom Director of Intelligence geleitet wurde. Außer ihm gab es noch vier Direktoren (Director of Information Control, Director of Public Relations, Director of Political Affairs und Director of Administrative Services) ${ }^{2}$ sowie einen Generalsekretär (Secretary General). Vor allem der Leiter der Verwaltungsdienste und der Generalsekretär übten die Dienststellenverwaltung aus. Dabei fielen die Sekretariatsdienste, die Postausgangskontrolle, Protokollangelegenheiten, Sprach- und andere Hilfsdienste in die Zuständigkeit des Generalsekretärs, während der Director of Administrative Services für Personal und Organisation zuständig war; ihm wurde auch der Adjutant General zugeteilt.

Die Zuständigkeit der vier anderen Direktoren lassen sich wie folgt beschreiben: Der Director of Intelligence nahm nachrichtendienstliche Aufgaben aller Art wahr, der Director of Information Control war für Presse, Publikationen, Film, Theater. Musik und Rundfunk, der Director of Public Relations für die Verbreitung von Informationen, die Auswertung der öffentlichen Meinung und die Beratung des Deputy Military Governor auf diesem Gebiet zuständig. Schließlich beschäftigte sich der Director of Political Affairs vorwiegend mit außenpolitischen Fragen, insbesondere den amerikanisch-deutschen „Beziehungen“, aber auch mit der Entnazifizierung.

Die Aufgaben der Fachverwaltung im engeren Sinne waren 16 Divisions zugewiesen. ${ }^{73}$ Diese lassen sich in fünf Gruppen zusammenfassen. Zur ersten Gruppe gehörten die Abteilungen Heer, Marine und Luftwaffe, verantwortlich für militärische Aufgaben einschließlich der Entmilitarisierung der deutschen Streitkräfte. Die zweite Gruppe, deren politische Grundsätze vom Assistant Deputy for Resources zu entwikkeln waren, umfaßte die Abteilung für Reparationen, Lieferungen und Rückerstattung, die Industrieabteilung sowie die Abteilung für Ernährung und Landwirtschaft. Für die Abteilungen für Handel und Gewerbe sowie für Finanzen war der (zweite) Assistant Deputy for Trade and Finance politisch zuständig. Der (dritte) Assistant Deputy for Public Services war verantwortlich für die Abteilungen für Recht, Verkehr, Nachrichtenwesen, öffentliche Sicherheit, Gesundheit und Wohlfahrt sowie Innere Verwaltung. Keinem der drei Assistant Deputies zugewiesen waren die Rechtsabteilung und die Abteilung für Kriegsgefangene und Verschleppte. Alle 16 Abteilungen wurden von Direktoren geleitet.

Keines der genannten Ämter war in Personalunion mit einem anderen verbunden. Als Generalsekretär fungierte Oberst Thomas W. Hammand, als Director of Administrative Services Oberst James B. Edmunds, als Director of Intelligence Brigadegeneral Thomas J. Betts, als Director of Public Relations Brigadegeneral Frank A. Allen, als Director of Information Control Brigadegeneral Robert A. McClure sowie als Director of Political Affairs Donald R. Heath.

\section{Ubersicht 5: Organisation und Personal von USGCC, 29. Juni 1945}

Naval Division

Army (Ground) Division

Air Division
V.Adm.
Maj.Gen.
Maj.Gen.

Robert L. Ghormley ${ }^{74}$

Ray W. Barker

Robert W. Harper

$72 \mathrm{Vgl}$. zum Director of Information Control USGCC 1944-45/11/13, zum Director of Public Relations USGCC $1944-45 / 11 / 10$ und zum Director of Administrative Services USGCC $1944-45 / 13 / 8$.

73 Vgl. Übersicht 5 und die General Orders Nr. 33 und Nr. 34 vom 29.6.1945. Vgl. auch die Notes from Discussion in 5/376-1/27.

$74 \mathrm{Vgl}$. USGCC $1944-45 / 5 / 5$. 
Reparations. Deliveries and Restitution Division Industry Division

Food and Agriculture Division

Trade and Commerce Division

Finance Division

Manpower Division

Transport Division

Communications Division

Public Safety Division

Public Health and Welfare Division

Civil Administration Division

Legal Division

Prisoners of War and Displaced Persons Division

$\begin{array}{ll}\text { Col. } & \text { Leslie W. Jefferson } \\ \text { Col. } & \text { James Boyd } \\ \text { Civ. } & \text { Loyd V. Steere (Actg) } \\ \text { Civ. } & \text { Frederick Winant (Actg) } \\ \text { Col. } & \text { Bernard Bernstein } \\ \text { Brig.Gen. } & \text { Frank J. McSherry } \\ \text { Brig.Gen. } & \text { John A. Appleton }{ }^{75} \\ \text { Brig.Gen. } & \text { Frank C. Meade } \\ \text { Col. } & \text { Orlando W. Wilson } \\ \text { Maj.Gen. } & \text { Morrison C. Stayer } \\ \text { Brig.Gen. } & \text { Luther S. Smith } \\ \text { Brig.Gen. } & \text { Edward C. Betts } \\ \text { Brig.Gen. } & \text { Stanley R. Mickelsen }\end{array}$

Mit dieser Organisationsstruktur nahm die USGCC mindestens der äußeren Form nach eine Gestalt an, die dem späteren Office of Military Government for Germany (US) recht ähnlich war. In diesem Sinne war es außerdem sehr wichtig, daß Clay den Leiter der G-5, SHAEF, General Clarence L. Adcock, zu seinem Stellvertreter ernannte. ${ }^{n}$

Für die künftige Entwicklung der amerikanischen Militärregierung war eine Entscheidung von besonderer Bedeutung, die Präsident Harry S. Truman am 16. Juni 1945 in Potsdam traf: Das War Department in Washington wurde allein zuständig für alle nicht außenpolitisch relevanten Fragen in Deutschland, während das State Department ausschlieBlich für rein außenpolitische Angelegenheiten verantwortlich wurde. $^{78}$

\section{Die Alliierte Kontrollbehörde ${ }^{79}$}

Zur Ausübung der obersten Regierungsgewalt und aller Machtbefugnisse, welche die Regierungen der Vereinigten Staaten von Amerika, der Sowjetunion, Großbritanniens und Frankreichs für Deutschland als Ganzes wahrnahmen, wurde - entsprechend den Planungen der Europäischen Beratenden Kommission - eine Alliierte Kontrollbehörde (Allied Control Authority) eingesetzt. Ihr oberstes Organ war der Kontrollrat (Control Council), der aus den vier Militärgouverneuren bestand und die Gesetzgebungskompetenz hatte. Alle Militärgesetze, Proklamationen, Befehle, Verordnungen, Bekanntmachungen, Vorschriften und Direktiven, die vom jeweiligen Oberbefehlshaber oder in dessen Namen für die jeweilige Zone erlassen worden waren, blieben jedoch auch weiterhin in Kraft. Der Kontrollrat, dessen Entscheidungen einstimmig getroffen werden mußten, hatte für eine „angemessene Einheitlichkeit" des Vorgehens der einzelnen Befehlshaber in ihren jeweiligen Besatzungszonen Sorge

\footnotetext{
${ }^{75} \mathrm{Vgl}$. USGCC 1944-45/11/12.

${ }^{76}$ GemäB General Order Nr.39 vom 24.7.1945 wurde Meade zum Assistant Deputy for Public Services ernannt; sein Nachfolger als Leiter der Communications Division wurde Brig.Gen. Carrol O. Bickelhaupt. Bereits mit General Order Nr. 43 vom 14.8.1945 wurde Bickelhaupt seinerseits durch Brig.Gen. Samuel M. Thomas ersetzt.

77 Ziemke: The U.S. Army, S. 402.

${ }^{78}$ Ebenda, S. 318.

${ }^{79}$ Vgl. Erdmann: Zeit der Weltkriege, S.697f;; Clay: Entscheidung in Deutschland, S. $123 \mathrm{ff}$. Neben den unter ACA zusammengefaBten Unterlagen vgl. auch USGCC 1944-45/15/2.
} 
zu tragen. Er traf im gegenseitigen Einvernehmen Entscheidungen über alle Deutschland als Ganzes betreffenden Grundsatzfragen.

Unter dem Kontrollrat wurden ein ständiger Koordinierungsausschuß (Coordinating Committee), der sich aus den vier Vertretern der Oberbefehlshaber zusammensetzte, und ein Kontrollstab (Control Staff) tätig, der aus folgenden Abteilungen (Directorates) bestand: Militär, Marine, Luftwaffe/Luftfahrt, Verkehr, Politik, Wirtschaft, Finanzen, Reparationen und Rückerstattung, Innere Angelegenheiten und Nachrichtenwesen, Rechtswesen, Kriegsgefangene und Verschleppte sowie Arbeitseinsatz. Jede Abteilung hatte vier Leiter, von denen je einer von jeder der vier Mächte ernannt wurde, wobei die vier Abteilungsleiter für den Bereich der jeweiligen Fachverwaltung das Directorate bildeten. Als Untergliederungen der Direktorate fungierten Arbeitsausschüsse (Principal Working Committees). Die Mitarbeiter im Kontrollstab rekrutierten sich sowohl aus Zivil- als auch aus Militärpersonen. In besonderen Fällen konnten auch in persönlicher Eigenschaft ernannte Angehörige anderer "Vereinter Nationen“ berufen werden.

Aufgabe des Koordinierungsausschusses war es vor allem, zur Entlastung des Oberbefehlshabers die Entscheidungen des Kontrollrats, soweit möglich, vorzubereiten. Der Kontrollstab hatte den Kontrollrat zu beraten, seine Beschlüsse auszuführen, sie an die zuständigen deutschen Behörden weiterzuleiten und deren laufende Tätigkeit zu überwachen. Schon mit Befehl vom 18. April 1945 war festgelegt worden, daß General Clay als Stellvertretender Militärgouverneur auch Vertreter der Vereinigten Staaten im Koordinierungsausschuß des Kontrollrates werden sollte.

Dem Sekretariat des Kontrollrats ${ }^{80}$ hatte man für den Fall der Errichtung deutscher Zentralbehörden die Aufgabe zugedacht, Weisungen an diese zu übermitteln. Eine gemeinsame Registratur der Kontrollbehörde wurde nicht geführt. Jede Macht verwaltete ihre Akten für sich, so daß aus dem amerikanischen Registratursystem nicht unbedingt auf die Verhältnisse bei den anderen drei alliierten Mächten geschlossen werden kann. Aktenverwaltende Stelle war auf seiten der Vereinigten Staaten das US Element Secretariat. Das Alliierte Sekretariat bestand aus den vier nationalen Sekretariaten der Siegermächte und hatte - abgesehen von den üblichen Sekretariatsdiensten - vor allem zu versuchen, Übereinstimmung über die Sitzungsniederschriften zu erzielen. Offizielle Verhandlungssprachen waren Englisch, Russisch und Französisch. Protokolle und sonstige Unterlagen (papers) wurden in der Sprache des jeweiligen Vorsitzenden erstellt und anschließend übersetzt.

Die Verbindung zu den Regierungen der anderen Mitgliedstaaten der Vereinten Nationen wurde durch die Ernennung von Militärmissionen beim Kontrollrat sichergestellt. Die Verwaltung des Gebiets von Groß-Berlin wurde einer interalliierten Behörde, der Kommandatura, unterstellt, die unter Leitung des Kontrollrats arbeitete und aus vier Kommandanten bestand, von denen jeweils einer abwechselnd als Hauptkommandant fungierte.

Da nur die Begriffe Coordinating Committee und Kommandatura unverwechselbar sind, sei zur Vermeidung von Mißverständnissen folgendes bemerkt: Den Begriff Allied Control Authority (Germany), der mit Alliierte Kontrollbehörde korrekt übersetzt wird, verwandte man, wenn alle Teile der Viermächteverwaltung, also der Kontrollrat (Control Council), die Vertreter (Deputies) der Oberbefehlshaber (Coordinating Committee) und deren Mitarbeiter (Control Staff, Kontrollstab) und das Alliierte Sekretariat (Allied Secretariat) bezeichnet werden sollten. Der kollek-

${ }^{80} \mathrm{Vgl}$. USGCC 1944-45/14/11. 
tive Singular Control Staff umfaßte die Summe der in Abteilungen (Directorates) und Ausschüssen (Committees) gegliederten Viermächteverwaltung.

Dieser Apparat arbeitete kompliziert und schwerfällig, weil überall - auch auf der untersten Ebene - alle vier Besatzungsmächte beteiligt waren. Da die Hauptaufgabe der Leiter der Fachabteilungen darin bestehen sollte, die entsprechenden deutschen Ministerien bzw. zentralen Einrichtungen zu überwachen, kann geschlossen werden, daß man damals offensichtlich noch davon ausging, sich eventuell einer deutschen Zentralverwaltung bedienen zu können. Ebenso deutlich ist jedoch, daß man das umständliche System der Kontrollbehörde auch für den Fall eines dezentralen Verwaltungsaufbaus in Deutschland für brauchbar hielt.

In der bereits erwähnten Erklärung vom 5.Juni 1945 wurde eine weitere Feststellung von den Alliierten gemeinsam getroffen, ${ }^{81}$ welche die Einteilung der Besatzungszonen zum Gegenstand hatte. Danach sollte Deutschland in den Grenzen vom 31. Dezember 1937 aus einer östlichen Zone der Union der Sozialistischen Sowjetrepubliken, einer nordwestlichen Zone Großbritanniens und einer süd(west)lichen Zone der Vereinigten Staaten von Amerika und einer (süd)westlichen Zone Frankreichs bestehen. Die Besatzungstruppen in jeder Zone unterstanden jeweils ihrem Oberbefehlshaber, jede der vier Mächte durfte nach eigenem Ermessen Hilfsverbände aus Streitkräften anderer alliierter Mächte aufnehmen, welche an den militärischen Operationen gegen Deutschland aktiv beteiligt waren.

Auf der Dreimächtekonferenz von Potsdam (Berlin) im Sommer $1945^{82}$ errichtete man außerdem einen Rat der Außenminister, dem neben den genannten vier Mächten vorübergehend noch China angehörte. Er tagte normalerweise in London und sollte Friedensverträge auch für Italien, Rumänien, Bulgarien, Ungarn und Finnland vorbereiten. In der Hauptsache diente der Rat jedoch der „Vorbereitung einer friedlichen Regelung für Deutschland“, damit „das entsprechende Dokument durch die für diesen Zweck geeignete Regierung Deutschlands angenommen werden kann, nachdem eine solche Regierung gebildet sein wird“.83

Die politischen Ziele der Besetzung Deutschlands, durch die sich der Kontrollrat leiten lassen wollte, wurden auf der Potsdamer Konferenz umfänglich bestimmt. Aus verwaltungsgeschichtlicher Sicht wichtig sind die Feststellungen, da $B$ das Gerichtswesen entsprechend den Grundsätzen der Demokratie und der Gerechtigkeit auf der Grundlage der Gesetzlichkeit und der Gleichheit aller Bürger vor dem Gesetz reorganisiert werden sollte. Für die Verwaltung Deutschlands wurden folgende Grundsätze festgestellt: Sie sollte dezentralisiert werden, um eine örtliche Selbstverwaltung zu entwickeln. Sie sollte zu diesem Zweck in ganz Deutschland nach demokratischen Grundsätzen durch Wahlausschüsse (Räte) so schnell wie möglich wiederhergestellt werden. Demokratische politische Parteien sollten zugelassen und Wahlen auf der Gemeinde-, Kreis-, Provinzial- und Landesebene durchgeführt werden. Politisch besonders bedeutsam war die Feststellung, daß bis auf weiteres „keine zentrale deutsche Regierung“ errichtet werden sollte. Vorgesehen waren indes ,einige wichtige zentrale deutsche Verwaltungsabteilungen“, an deren Spitze Staatssekretäre stehen sollten. Hierbei war an die Bereiche Finanzen, Verkehr, Außenhandel und Industrie gedacht. Diese Abteilungen sollten nach den Weisungen des Kontrollrats tätig werden, wurden jedoch nicht errichtet. Von den wirtschaftlichen Grundsätzen sind die Vernichtung des

${ }^{81}$ Eschenburg: Jahre der Besatzung, S. 46f.

${ }^{82}$ Foreign Relations, Berlin. Vgl. USGCC 1944-45/6/12; AG 1945-46/66/1; AG 1944-45/79/4; AG 1945-46/80/6.

${ }^{83}$ Erdmann: Zeit der Weltkriege, S.620. 
deutschen Kriegspotentials, die Dezentralisierung des deutschen Wirtschaftslebens sowie die Entwicklung der Landwirtschaft und der sogenannten Friedensindustrie hervorzuheben. Bedeutsam war ferner die Feststellung, daß Deutschland während der Besatzungszeit als wirtschaftliche Einheit zu betrachten sei. ${ }^{84}$ Das gesamte deutsche Wirtschaftsleben sollte alliierter Kontrolle unterworfen sein. Man einigte sich weiter über eine „ordnungsgemäße Überführung deutscher Bevölkerungsteile“ aus Ost- und Südosteuropa. ${ }^{85}$ SchlieBlich verständigte man sich auch auf die Grundsätze, nach denen Deutschland Reparationen auferlegt werden sollten. ${ }^{86}$

In der ersten Sitzung des Kontrollrats am 30. Juli 1945 in Berlin wurde auf Vorschlag Eisenhowers festgelegt, das Gremium möge sich im Abstand von jeweils zehn Tagen am 10., 20. und 30. Tag eines jeden Monats treffen. Die Oberbefehlshaber beschlossen ferner, den Vorsitz im monatlichen Wechsel zu führen. Der jeweilige Vorsitzende nach dem Alphabet zunächst General Eisenhower - stellte auch den Hauptsekretär (Chief Secretary) für die Sitzungen im betreffenden Monat.

Beim zweiten Treffen der vier Oberbefehlshaber am 10. August 1945 im Gebäude des ehemaligen Volksgerichtshofes wurde die Vorlage über die endgültige Organisation des Kontrollrats gebilligt, über die sich die Stellvertreter der Oberbefehlshaber (für die amerikanische Seite also General Clay) bei ihrer Sitzung am 1. August 1945 geeinigt hatten. Danach sollte der Koordinierungsausschuß entscheiden, welches Gremium bestimmte Sachfragen zu behandeln hatte. In der Praxis wurden die meisten Fachfragen auf der Ebene der Abteilungen (Directorates), Angelegenheiten mittlerer Bedeutung im Koordinierungsausschuß behandelt und nur bei höchst kontroversen Streitpunkten und in Grundsatzfragen schaltete man den Kontrollrat selbst ein. Durch die Proklamation Nr. 1 vom 30. August 1945 unterrichtete der Kontrollrat in seinem Amtsblatt Nr.1 vom 29. Oktober 1945 die deutsche Bevölkerung über seine Errichtung. Alle (Militär-)Gesetze, Proklamationen, Befehle, Verordnungen, Bekanntmachungen, Vorschriften und Direktiven, die von einem der vier Oberbefehlshaber oder in dessen Namen für die jeweilige Besatzungszone erlassen worden waren, blieben wie bereits erwähnt - in Kraft. Entsprechende Regelungen des Kontrollrats für Deutschland als Ganzes wurden seitdem im Amtsblatt des Kontrollrats veröffentlicht. In einem Ergänzungsblatt Nr. 1 wurde eine "Sammlung von Urkunden betreffend die Errichtung der Alliierten Kontrollbehörde" dem Amtsblatt beigegeben.

\section{Die Konsolidierung von USGCC}

Mit der Umwandlung der USGCC von einer Planungsgruppe in die obere Behörde für die Militärregierung in Deutschland, der Umstrukturierung von ETOUSA in eine Kommandobehörde mit Sitz in Frankfurt am Main unter gleichzeitiger Umbenennung zum 1. Juli in USFET und der Auflösung von SHAEF Mitte Juli 1945 war die geschilderte Umstrukturierung von USGCC sachlich, zeitlich und personell eng verbunden. Die USGCC zog ab 10. Juli von Frankfurt-Höchst nach Berlin um, wobei einige Abteilungen Außenstellen in Frankfurt behielten. In den folgenden Monaten des Jahres 1945 kam es zu weiteren sehr wichtigen personellen wie organisatorischen Veränderungen. ${ }^{87}$ So hatte der Legal Adviser, Charles Fahy, bereits am 20.Juli 1945 die Lei-

\footnotetext{
84 Ebenda, S. 621.

85 K.-D. Henke: Weg nach Potsdam, S. 68.

${ }^{86}$ Erdmann: Zeit der Weltkriege, S.621 ff.

87 USGCC 1944-45/3/2; USGCC 1944-45/12/5.
} 
tung der Legal Division übernommen. Der bisherige Direktor der Rechtsabteilung. General Edward C. Betts, war innerhalb der Rechtsabteilung zum Stellvertretenden Direktor für die Verfolgung von Kriegsverbrechen ernannt worden. ${ }^{88}$

Politisch bedeutsam war vor allem die Errichtung einer Abteilung für die Untersuchung von Kartellen und Auslandsguthaben (Division of Investigation of Cartels and External Assets). ${ }^{89}$ Diese neue Abteilung war bisher Teil der Finanzabteilung und wurde von deren bisherigem Leiter Oberst Bernard Bernstein geführt. Ganz offensichtlich wurde dieser Offizier wegen seiner finanzpolitischen Ideen, die vom Morgenthau-Plan beeinflußt waren, ${ }^{00}$ von der Leitung der so wichtigen Finanzabteilung entbunden, doch konnte oder wollte Clay ihn nicht völlig kaltstellen.

Noch wichtiger war die Errichtung des Control Office, Office Chief of Staff, an Stelle des bisher schon als Teil des Stabsamtes bestehenden Reports Control Office. ${ }^{91}$ Das Control Office entwickelte sich später zur entscheidenden Koordinierungsstelle nicht nur - wie es offensichtlich ist - für organisatorische Fragen, sondern auch für politische Grundsatzentscheidungen.

An die Spitze trat Oberst William Whipple als Control Officer. Die mächtige Stellung, die Control Office und Control Officer bis zur Auflösung von OMGUS ausübten, wird bereits in der Aufgabenbeschreibung deutlich, die in General Order Nr.53 vom 13. September 1945 unter Abschnitt III enthalten ist. Danach hatte der Kontrolloffizier die Durchführung der Programme der Fachabteilungen von USGCC ständig zu überprüfen. Er hatte dabei das Recht, Berichte in dem ihm erforderlich erscheinenden Umfang anzufordern. Er war für die Personalausstattung verantwortlich, um eine gute Koordinierung und Überwachung des gesamten Verwaltungs- und Berichtssystems der Militärregierung zu ermöglichen. Damit wollte man auch eine möglichst vollständige Unterrichtung Washingtons gewährleisten. Auf wirtschaftlichem Gebiet wurde ein besonderer Control Officer (I.G. Farbenindustrie A.G.) eingesetzt, dessen Funktion mit Allgemeiner Verfügung Nr. 58 vom 29. September 1945 ebenfalls umfänglich umschrieben wurde.

Der amerikanische Anteil (US Element) am Kontrollrat und USGCC stimmten im Verwaltungsaufbau in großen Zügen überein. Um nach außen eine völlige Identität zu erreichen, wurde in General Order Nr.33 vom 29. Juni 1945 angeordnet, daB drei Angehörige von USGCC, nämlich der Director of Political Affairs, der Assistant Deputy for Resources und der Assistant Deputy of Public Services im Kontrollrat als Directors of Political, Economics bzw. of Internal Affairs and Communications Directorate auftreten sollten. Unabhängig von dieser Konstruktion war die Organisation von USGCC in einem für die Aufgabenerfüllung wesentlichen Gebiet, nämlich die Vorbereitung einer künftigen deutschen Regierungsorganisation, freilich unzulänglich; dies hat in der Folge zu deutlicher Kritik gerade durch amerikanische Experten geführt. ${ }^{92}$

${ }_{88}$ General Order Nr. 38 vom 20.7.1945.

${ }^{89}$ General Order Nr. 52 vom 12.9.1945. Vgl. 5/376-1/27.

90 Gimbel: Amerikanische Besatzungspolitik, S. 19.

${ }^{91}$ General Order Nr. 53 vom 13.9.1945; AG 1944-45/69/6.

92 "It had no subdivision charged with the main job of dealing with the German governmental structure" (Zink: The United States in Germany, S.27). 


\section{Die Errichtung von OMGUS und OMGUSZ}

Zwischen USGCC und G-5, USFET, hatte es mehrere Monate lang unfruchtbare Rivalitäten gegeben. General Clay hatte stets die Auffassung vertreten, daß die Hauptaufgabe der Amerikaner in Deutschland - die Wiederherstellung einer Zivilverwaltung auf demokratischer Grundlage - nicht fünf Stabsabteilungen und einem Armeekommando (also USFET) überlassen werden könne; die Militärregierung müsse vielmehr zu einem guten Teil aus Zivilangestellten bestehen. Diese Auffassung wurde von Eisenhower stets geteilt. ${ }^{93}$

Nach Verlegung des Hauptquartiers von USGCC nach Berlin ab 30. Juli 1945 waren Außenstellen in Frankfurt-Höchst zurückgeblieben. Die Berliner Stellen bildeten das Headquarters US Group, Control Council (Germany) (Main) - die bis zum 20. August in Höchst fortbestehenden Außenstellen führten statt des „Main“ ein ,.Rear” in der Behördenfirma. ${ }^{94}$ Durch General Order Nr.59 vom 29.September 1945 wurde USGCC in Office of Military Government for Germany (US) umbenannt. ${ }^{95}$ Im Sinne einer reibungslosen Zusammenarbeit zwischen allen für die Besatzungsverwaltung zuständigen Stellen und Einheiten wurde die Abteilung G-5, USFET, mit Ausnahme einer kleinen Gruppe, deren Verbleib in Frankfurt notwendig erschien, ebenfalls nach Berlin verlegt und dort mit dem Office of Military Government for Germany (US) vereinigt. Diese neue Einrichtung, deren Umbenennung mit Wirkung vom 1. Oktober 1945 verfügt wurde, war bald unter der Abkürzung OMGUS allgemein bekannt. Die in Frankfurt am Main zurückbleibenden Teile von G-5, USFET, wurden mit derselben Anordnung umbenannt in Office of Military Government (US Zone) ${ }^{90}$ - besser bekannt als OMGUSZ - und hatten die Aufgabe, die von OMGUS beschlossene Politik in der amerikanischen Zone auszuführen. Zum Chef dieser Frankfurter Dienststelle wurde Clays Jugendfreund, Generalmajor Adcock, ernannt. Adcock verfügte über einen Adviser for German Affairs und vier zentrale Einrichtungen (Central Offices): Plans Branch, Editing Committee, Reports and Information Branch und eine Personnel and Equipment Branch. Die regionalen Untereinheiten von OMGUSZ wurden zum Office of Military Government Bavaria und zum Office of Military Government (Western District). ${ }^{97}$ Man kann diese Änderungen auch als Zeichen für die Absicht Clays werten, die Verantwortung für Deutschland sehr bald in ausschlieBlich zivile Hände, d. h. also in den Geschäftsbereich des Außenministeriums zu überführen. General Eisenhower hatte es gegenüber seinen Armeekommandeuren bereits am 1. November 1945 als das erste Ziel der Armee nach der Kapitulation Deutschlands bezeichnet, nach Herstellung der öffentlichen Ordnung die amerikanische Aufsicht über deutsche Angelegenheiten zivilen Stellen zu übertragen. Wäre es nach Eisenhowers und Clays Wünschen gegangen, hätte man den Übergang der Verantwortung für Deutschland in zivile Verwaltung innerhalb von 24 Stunden durchführen können. ${ }^{98}$

Sieben Abteilungen von OMGUS (Internal Affairs and Commuinications, Displaced Persons, Economics, Legal, Finance, Transportation, Manpower) unterhielten weiterhin Außenstellen in Frankfurt. Außerdem richtete man bei USFET wieder eine ziemlich umfangreiche - Abteilung G-5 ein, welche diejenigen Aufgaben übernehmen

\footnotetext{
93 Vgl. Clay Papers, S. 77 f.

94 General Order Nr. 41 vom 30.7.1945.

$95 \mathrm{Vgl}$. Military Government Bulletin Nr. 10 vom 29.9.1945, S. $7 \mathrm{f}$.

96 3/148-3/9; Ziemke: The U. S. Army, S. 402 f.; Zink: The United States in Germany, S. 29.

97 Steger: Clays Stabskonferenzen, S. 120 f.; Ziemke: The U. S. Army, S. $401 \mathrm{ff}$.

98 Ebenda, S. $403 \mathrm{ff}$.
} 
sollte. die aus der Sicht des Militärs nicht in den Bereich des gleichsam als zivil angesehenen OMGUS fallen konnten. Tatsächlich wurden die Funktionen dieser neuen G-5. USFET, im wesentlichen auf Verbindungs- und Koordinierungsaufgaben sowie in Zusammenarbeit mit der Weltflüchtlingsorganisation UNRRA - auf die Angelegenheiten der Displaced Persons beschränkt. Darüber hinaus übernahm die neue G-5. USFET, von OMGUSZ die Zuständigkeit für die Betreuung amerikanischer Bürger außerhalb Deutschlands, soweit diese nicht Angehörige des Militärs waren.

Im Grundsatz wurde die Organisation der USGCC von OMGUS beibehalten: ${ }^{99} \mathrm{Die}$ Militärregierung bestand aus dem Office of the Deputy Military Governor (Executive Office), vier Fachämtern und acht bzw. neun Fachabteilungen. Die entscheidende Änderung bestand dann in der Auflösung der Ämter der Assistant Deputies, die mit Wirkung vom 15. Oktober 1945 in Kraft trat. ${ }^{100}$ Danach bestanden das Office of the Deputy Military Governor (Clay), das Office of the Chief of Staff (Bryan L. Milburn), das Office of the Secretary General (David Marcus), zu dem auch der amerikanische Anteil am Sekretariat der Kontrollbehörde gehörte, das Control Office (William Whipple), das Personnel Office (G-1, Arthur H. Onthank) und das Office of the Director for Administrative Services (James B. Edmunds), zu dem u. a. das Verwaltungsbüro des amerikanischen Anteils des Kontrollrats und das Amt des Adjutant General gehörten. Der Generalsekretär, der Control Officer, der Personnel Officer ${ }^{101}$ und der Direktor der Verwaltungsdienste waren unter dem Chef des Stabes zum Executive Office zusammengefaßt. Hinzu traten vier Fachämter (Functional Offices): das Amt des Direktors für nachrichtendienstliche Aufgaben (Intelligence) ${ }^{102}$ (G. Bryan Conrad), das Amt des Information Control Services ${ }^{103}$ (Robert A. McClure), das Amt für Öffentlichkeitsarbeit (Office of Public Relations) ${ }^{104}$ (Francis V. Fitzgerald) und das Amt des Direktors für politische Angelegenheiten (Donald R. Heath). Ebenso wie der Naval Adviser, Robert L. Ghormley, ${ }^{105}$ blieb auch der Political Adviser, Robert D. Murphy, Clay unmittelbar zugeordnet. Klarheit über die Aufgabentrennung der beiden für politische, d. h. im wesentlichen für außenpolitische Fragen zuständigen Mitarbeiter Clays, dem Political Adviser, Murphy, und dem Direktor für politische Angelegenheiten, Heath, bestand offensichtlich nicht. Jedenfalls hatten die Advisers, zu denen auch ein Economic Adviser, ein Finance Adviser und ein Legal Adviser gehörten, eine vergleichsweise unabhängige Stellung. Die drei zuletzt genannten waren jedoch im Unterschied zum Naval Adviser und zum Political Adviser gleichzeitig Direktoren von Fachabteilungen: So dienten William H. Draper ${ }^{106}$ sowohl als Economic Adviser als auch als Direktor der Wirtschaftsabteilung, Joseph N. Dodge ${ }^{107}$ als Finance Adviser als auch als Direktor der Finanzabteilung und Charles Fahy ${ }^{108}$ als Legal Adviser und als Direktor der Rechtsabteilung. $\mathrm{DaB}$ diese drei Leiter von Fachabteilungen gleichzeitig als fachliche Berater des Stellvertretenden Militärgouverneurs fun-

\footnotetext{
99 5/370-2/18; 5/376-1/27; USGCC 1944-45/10/7-8; USGCC 1944-45/11/1-13; USGCC 1944 45/11/15; AG 1944-45/70/3-4.

100 General Order Nr.63 vom 9.10.1945. Vgl. 5/14-1/45.

101 AG 1945-46/69/11.

102 AG $1945-46 / 69 / 17-18$.

103 AG 1945-46/64/8.

104 AG 1945-46/69/12.

105 AG 1945-46/64/3.

106 Zu Draper vgl. Clay: Entscheidung in Deutschland, S. 20 ff.

107 Zu Dodge vgl. ebenda, S. 232; Clay Papers, S. 79.

108 Zu Fahy vgl. Clay: Entscheidung in Deutschland, S.87.
} 
gierten, ist sicherlich in einer persönlichen Wertschätzung durch Clay begründet gewesen; außerdem dürfte Clay damit auch sachliche Prioritäten gesetzt haben.

Unter den Fachabteilungen nahm wie bisher die Armed Forces Division unter Generalmajor Robert W. Harper eine Sonderstellung ein. Weiterhin gab es eine Transport Division unter Brigadegeneral John A. Appleton, ${ }^{109}$ eine Manpower Division unter Brigadegeneral Frank J. McSherry, ${ }^{110}$ eine Internal Affairs and Communications Division unter Brigadegeneral Frank C. Meade, ${ }^{11}$ eine Prisoners of War and Displaced Persons Division unter Brigadegeneral Stanley R. Mickelsen ${ }^{112}$ sowie eine Investigations of Cartels and External Assets Division unter Oberst Bernard Bernstein. Hinzu kam schließlich das Kommando des Hauptquartiers (Headquarters Command).

In der Economics Division, OMGUS, $1{ }^{13}$ wurden die folgenden Einheiten von USGCC zusammengefaßt: das Office of Assistant Deputy for Resources, die Industry Division, die Food and Agriculture Division, die Trade and Commerce Division sowie zwei Unterabteilungen der Reparations, Deliveries and Restitution Division (die Reparations and Restitution Branch und die Fine Arts and Archives Branch). In der neuen Finance Division ${ }^{14}$ vereinigte man das Office of the Assistant Deputy for Trade and Finance. die Unterabteilung Property Control der Reparations, Deliveries and Restitution Division und das Office des Control Officers (I.G. Farbenindustrie A. G.). Schließlich umfaßte die neue Internal Affairs and Communications Division das Office of the Assistant Deputy for Public Services, die Communications Division, die Civil Administration Division, die Public Safety Division und die Public Health and Welfare Division.

Folgende Mitarbeiter von OMGUS bekleideten Funktionen in den Direktoraten der Alliierten Kontrollbehörde: Der Direktor der Armed Forces Division vertrat die Vereinigten Staaten im Allied Directorate - Air, der Deputy Director of Ground Forces Division im Allied Directorate - Naval, der Direktor der Wirtschaftsabteilung im Allied Directorate - Economics, der Leiter der Reparations Branch im Allied Directorate - Reparations, Deliveries, Restitution, der Leiter der Finanzabteilung im Allied Directorate - Finance, der Leiter der Verkehrsabteilung im Allied Directorate -Transport, der Leiter der Internal Affairs and Communications Division im Allied Directorate - Internal Affairs and Communications, der Leiter der Rechtsabteilung im Allied Directorate - Legal, der Leiter der Prisoners of War and Displaced Persons Division im Allied Directorate - Prisoners of War and Displaced Persons sowie der Political Adviser im Allied Directorate - Political.

\section{Ubersicht 6: Organisation und Personal von OMGUS, Oktober 1945}

\section{Ofice of the Deputy Military Governor}

Deputy Military Governor

Special Assistant to the Deputy Military Governor

$\begin{array}{ll}\text { Li.Gen. } & \text { Lucius D.Clay } \\ \text { Li.Col. } & \text { R.R.Bowie } \\ \text { Lt.Col. } & \text { D.H. McLean } \\ \text { Maj.Gen. } & \text { Oliver P. Echols } \\ \text { Brig.Gen. } & \text { Bryan L. Milburn }\end{array}$

Assistant Deputy Military Governor

Chief of Staff

Brig.Gen.

\footnotetext{
109 AG 1945-46/69/13.

$110 \mathrm{Vgl}$. Clay: Entscheidung in Deutschland, S. 323; Fichter: Besatzungsmacht und Gewerkschaften, S. $46 \mathrm{ff}$.

111 AG 1945-46/68/1: AG 1945-46/69/7.

112 AG 1945-46/70/5.

113 AG 1945-46/68/2; AG 1945-46/69/3.

114 AG 1945-46/69/4.
} 
Sectetary General

US Element of the Allied Secretariat

Acting US Secretary

Assist. Secretary

Control Office, Control Officer

Personnel Office. Chief

Office of the Director of Administrative Services,

Director

Administrative Bureau, ACA, Chief

Adjutant General

Office of the Director of Intelligence, Director

Deputy Director

Executive Officer

Office of Information Control Services, Director

Executive Officer

Office of Public Relations Services, Director

Office of the Political Adviser for Germany, Adviser

Office of the Director of Political Affairs, Director

Denazification Branch, Chief

Intelligence Branch, Chief

Economics Branch, Chief

American Interests Branch, Chief

\section{Armed Forces Division}

Director

Deputy Director

Disarmament Branch, Chief

Demobilization Branch, Chief

\section{Economics Division}

Director

Deputy Director

Deputy Director

Policy and Control Branch, Chief

Food and Agriculture Branch, Chief

Restitution Branch, Chief

Industry Branch, Chief

\section{Finance Division}

Director

Deputy Director

Financial Institutions Branch, Chief

Foreign Exchange and Blocking Control Branch, Chief

Financial Intelligence and Liaison Branch, Chief

Currency Branch, Chief

Accounts and Audits Branch, Chief

Property Control Branch, Chief

I. G. Farben Control Branch, Chief

\section{Transport Division}

Director

Deputy Director

Road Branch, Chief

Rail Branch. Chief
Col.

David Marcus

Lt.Col

R. Birdsall

Lt.Col.

Charles E.Sands

Col.

Col.

Lt.Col.

Col.

Col.

Col.

Brig.Gen.

Col.

Col.

Brig.Gen. Robert A.McClure

Lt.Col.

Lt.Col.

Lt.Col.

Amb.

Civ.

Civ.

Col.

Civ.

Civ.

William Whipple

Arthur H. Onthank

James L. Williams

James B. Edmunds

F.W.Drury

Bruce Easley

G.Bryan Conrad

Theodore J.Koenig

T.F. Bogart

R. K. Fried

Duncan-Clark

James L. Williams

Robert D.Murphy

Donald R. Heath

J.J.Muccio

W.D. Hohenthal

Loyd V.Steere

A. D. Hodgdon

Maj.Gen.

Col.

Robert W. Harper

Col.

F.M. Albrecht

Col.

R.W. Yates

T.B. Whitted

Brig.Gen. William H.Draper

Civ.

Civ.

F.L. Devereux

Laird Bell

Civ.

Brig.Gen.

Col.

Col.

S. Wennberg

Hugh B. Hester

Leslie W. Jefferson

James Boyd

Civ.

Col.

Civ.

Civ.

Civ.

Lt.Col.

Col.

Col.

Col.

Joseph M. Dodge

David L. Robinson

Walter Lichtenstein Jack Bennett

A. M. Kamarck

H.D. Cragon

M. S. Hudtluff

C.S. Reid

E.S.Pillsbury

Brig.Gen. John A. Appleton

Col. J.B.Hughes

Li.Col. J.L.S.Snead

Lt.Col. W. G. White 
Water Transport Branch, Chief

Movements and Traffic Branch. Chief

Manpower Division

Director

Deputy Director

hternd Afiairs and Communications Division

Director

Deputy Director

Civil Administration Branch, Deputy Director

Public Health and Welfare Branch. Chief

Public Welfare Section, Chief

Education and Religious Affairs Section

Education Section, Chief

Religious Section, Chief

Communications Branch, Chief

Public Safety Branch. Chief

\section{Legal Division}

Director and Legal Adviser

Associate Director

Associate Director

Deputy Director for War Crimes

Deputy Director for Administration

Legal Advice Branch, Chief

Justice Ministry Branch, Chief

Judicial Branch, Acting Chief

\section{Prisoner of War and Displaced Persons Division}

Director

Displaced Persons Branch, Chief

Prisoner of War Branch, Chief

Investigation of Cartels and External Assets Division

Director

Deputy Director

\author{
L.t.Col. D. R. Neff \\ Col. T.C. Wenzlaff \\ $\begin{array}{ll}\text { Brig.Gen. } & \text { Frank J. McSherry } \\ \text { Civ. } & \text { Leo R. Werts }\end{array}$
}

Brig.Gen. Frank C. Meade

Col. S.R.Brentnall

Civ. James K. Pollock

Maj.Gen. Morrison C.Stayer

Lt.Col. E.F.Witte

Maj. J.W.Taylor

Maj. M.M.Knappen

Brig.Gen. S.M.Thomas

Col. Orlando W. Wilson

$\begin{array}{ll}\text { Civ. } & \text { Charles Fahy } \\ \text { Civ. } & \text { J.Warren Madden } \\ \text { Civ. } & \text { H.Phleger } \\ \text { Brig.Gen. } & \text { Edward C.Betts } \\ \text { Col. } & \text { J.B.Marsh } \\ \text { Lt.Col. } & \text { S. Bradford } \\ \text { Col. } & \text { E.L. McLendon } \\ \text { Lt.Col. } & \text { Frank Seydel }\end{array}$

Brig.Gen. Stanley R. Mickelsen

Lt.Col. James P. Abbott

Lt.Col. C.H.Ott

Col. Bemard Bernstein

Civ. R. A.Nixon

Unabhängig davon, ob man die Kritik am Fehlen einer eigenständigen Abteilung für die Vorbereitung des Aufbaus einer neuen deutschen Regierung sowie einer Kulturabteilung für berechtigt hält, ist insgesamt mit Sicherheit eine Straffung des Verwaltungsaufbaus festzustellen. Allerdings führte dies nicht dazu, daß Organisation und Personaleinsatz für längere Zeit unverändert blieben. Die erste bedeutende Organisationsänderung auf der Ebene der Fachabteilungen war die Auflösung der erst Mitte September 1945 errichteten Division of Investigation of Cartels and External Assets Mitte Dezember 1945. ${ }^{115}$ Außerdem wurde die Dienststelle des I.G. Farben Control Officers mit Wirkung vom 15. Dezember 1945 aufgelöst. ${ }^{16}$ Die Funktionen dieser Stellen übernahmen die Finance Division bzw. die Economics Division. Anfang Februar 1946 wurde innerhalb des Control Office eine Rechnungsprüfungsbehörde (Audit Office) errichtet. ${ }^{117}$ Am 25 . Februar 1946 erfolgte die Einsetzung eines Intelligence Coordinating Committee, dem unter dem Vorsitz des Director of Intelligence weitere

115 General Order Nr. 73 vom 14.12.1945.

116 General Order Nr. 74 vom 14.12.1945.

117 General Order Nr. 4 vom 6.2.1946. 
neun OMGUS-Bedienstete aus mehreren Divisions, Branches und Sections angehörten. ${ }^{11 \times}$ Dadurch wurde sichergestellt, daß nachrichtendienstliche Aufgaben unter unmittelbarer Aufsicht Clays straff koordiniert wurden.

Das weitreichende Aufgabenfeld des amerikanischen Stellvertretenden Militärgouverneurs in Deutschland machte selbstverständlich einen intensiven und kontinuierlichen Informationsaustausch zwischen ihm und seinem Beraterstab notwendig. In der USGCC hatte sich „aus anfangs noch informellen Gesprächen“, die Wickersham mit seinen engsten Beratern geführt hatte, die Einrichtung von regelmäßig stattfindenden Stabskonferenzen entwickelt. ${ }^{119}$ Clay übernahm diese Einrichtung und benutzte sie. um seinem eigenen Informationsbedürfnis und der Unterrichtung seiner Mitarbeiter über aktuelle politische Entscheidungen auf verschiedenen Ebenen sachgerecht zu entsprechen. Auch auf ein umfassendes Berichtswesen zur Unterrichtung der vorgesetzten Stellen in Washington, der Angehörigen von OMGUS, aber auch der Öffentlichkeit hat Clay von Anfang an großen Wert gelegt.

Nicht alle seine Mitarbeiter werden Clay bescheinigt haben, daß Instanzenzug und Geschäftsgang bei OMGUS sonderlich effektiv gewesen seien. Es ist nämlich zu bedenken, daß unabhängig von der nicht sonderlich übersichtlichen Leitungsebene die Abteilungen (Divisions) sich mindestens in Unterabteilungen (Branches) und Referate (Sections) gliederten, wobei teilweise die Abteilungsleiter (Directors), seltener die Unterabteilungsleiter (Chiefs of Branch) über eigene Mitarbeiterstäbe, die man wiederum Offices nannte, und einen Stellvertreter (Deputy) verfügten. Auch wenn die amerikanische Militärregierung etwa im Vergleich zur britischen Besatzungsverwaltung nicht als personell überbesetzt angesehen werden kann, ${ }^{120}$ so werden die Klagen über einen schwerfälligen Geschäftsgang durchaus verständlich, wenn man bedenkt, da $B$ im November 19454024 Amerikaner, etwa 8000 deutsche Zivilbedienstete und 1200 Kriegsgefangene für die amerikanische Militärregierung in Deutschland arbeiteten.

Die von den Alliierten vorgesehenen deutschen Institutionen zur Gewährleistung der wirtschaftlichen Einheit des Vier-Zonen-Deutschlands - die deutschen Staatssekretariate unter Aufsicht des Kontrollrats in Berlin - wurden vor allem aufgrund französischer Obstruktion niemals errichtet. ${ }^{121}$ General Clay bemühte sich daher um eine deutsche koordinierende Verwaltungsspitze wenigstens für die amerikanische Zone, um eine gewisse Einheitlichkeit oberhalb der Länderebene zu erreichen, ohne dabei von den föderalistischen Grundsätzen der amerikanischen Besatzungspolitik abzuweichen. So entstand ein Länderrat für das amerikanische Besatzungsgebiet, in dem die Regierungschefs der drei Länder der amerikanischen Zone, später auch der Bremer Senatspräsident, zusammenarbeiteten, um eine gewisse Einheitlichkeit der deutschen Verwaltungsätigkeit oberhalb der Länderebene zu sichem. ${ }^{12}$ Am 17. Oktober 1945 hielt dieser Länderrat seine erste Sitzung in Stuttgart ab. ${ }^{123}$ Als Pendant innerhalb der amerikanischen Besatzungsverwaltung wurde ein Verbindungsstab beim Länderrat geschaffen, dessen Leiter, James K. Pollock, am 25. Oktober 1945 in Stuttgart, dem ständigen Sitz des Länderratssekretariats, eintraf und mit dem Aufbau einer kleinen

\footnotetext{
118 General Order Nr.9 vom 25.2.1946.

119 Steger: Clays Stabskonferenzen, S. 119.

120 Friedmann: Allied Military Government, S.36.

121 Benz: Von der Besatzungsherrschaft, S. 29.

122 Vogel: Westdeutschland 1, S. 57 ff.; Eschenburg: Jahre der Besatzung, S.85f. Vgl. Germany under occupation, S. 102 ff.; USGCC 1944-45/13/11.

$123 \mathrm{Vgl}$. Akten zur Vorgeschichte 1, S. $125 \mathrm{ff}$.
} 
Behörde begann, die unter dem Namen Regional Government Coordinating Office (RGCO) bekannt wurde. Aufgrund des engen Vertrauensverhältnisses zwischen Clay und Pollock ${ }^{124}$ wurde das RGCO sehr schnell zu einer der wichtigsten Dienststellen innerhalb des amerikanischen Besatzungsapparates, da die Weisungen an die Landesregierungen grundsätzlich über das Regional Government Coordinating Office geleitet werden muBten.

Obere Besatzungsbehörden wie das Regional Government Coordinating Office bestanden außerdem in Form des Counsel for War Crimes, zu dessen Leiter (US Chief of Counsel for War Crimes) bereits am 22. Mai 1945 Robert H. Jackson emannt worden war. Dieser Counsel for War Crimes war dem Bereich des Director of Intelligence ebenso zugeordnet worden wie das im Juni 1945 in der Nähe von Kassel bei Hessisch Lichtenau/Fürstenhagen errichtete Ministerial Collecting Center (MCC), ${ }^{125}$ die am 2. August 1945 gegründete Field Intelligence Agency, Technical (FIAT) ${ }^{126}$ und das Berlin Document Center (BDC).

Ubersicht 7: Organisation von OMGUS, November 1945

OFFICE of MILITARY GOVERNMENT for GERMANY (US)

- BERLIN -

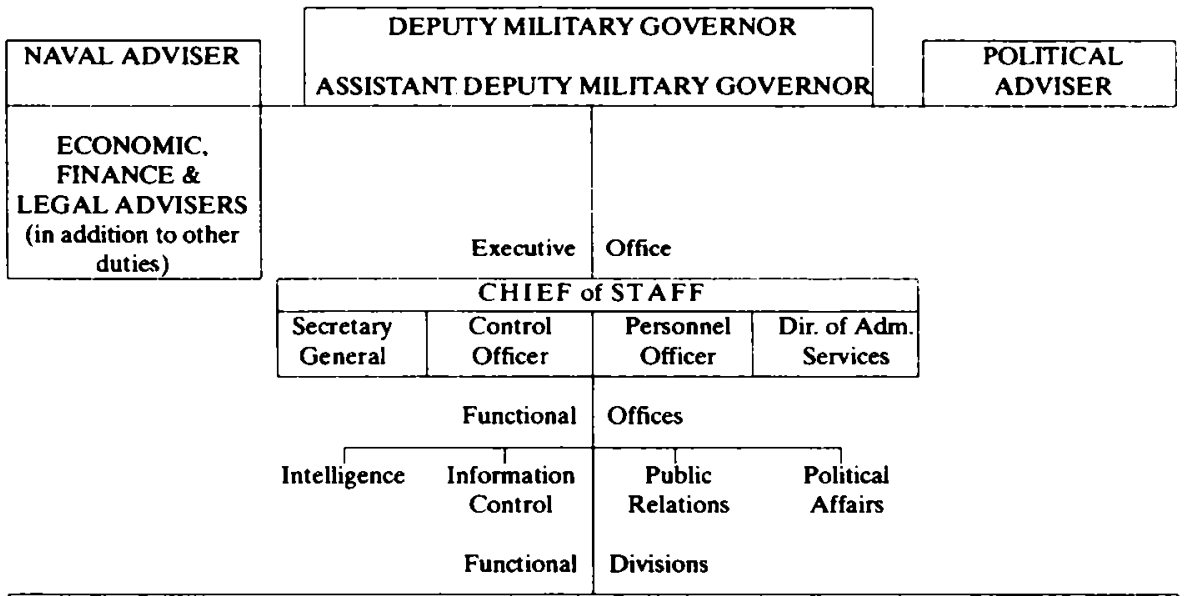

ARMED FORCES

ECONOMICS Division

FINANCE Division

TRANSPORT Division

MANPOWER Division
INTERNAL AFFAIRS \& COMMUNICATIONS Division

LEGAL Division PRISONERS of WAR \&DISPLACED PERSONS Division INVESTIGATIONS of CARTELS \& EXTERNAL ASSETS Division

124 Vgl. die Korrespondenz zwischen Clay und Pollock in 11/42-3/1.

125 Borm: Ministerial Collecting Center, S. 237 ff.; USGCC 1944-45/10/3, 17/8309-44.

126 USGCC 1944-45/10/6; USGCC 1944-45/13/9; AG 1945-46/64/11. Vgl. Clay Papers, S. 39 f. 
Ubersicht 8: Organisation von OMGUS und OMGUSZ, November 1945

Office of Military Govemment for Gemany

(US)

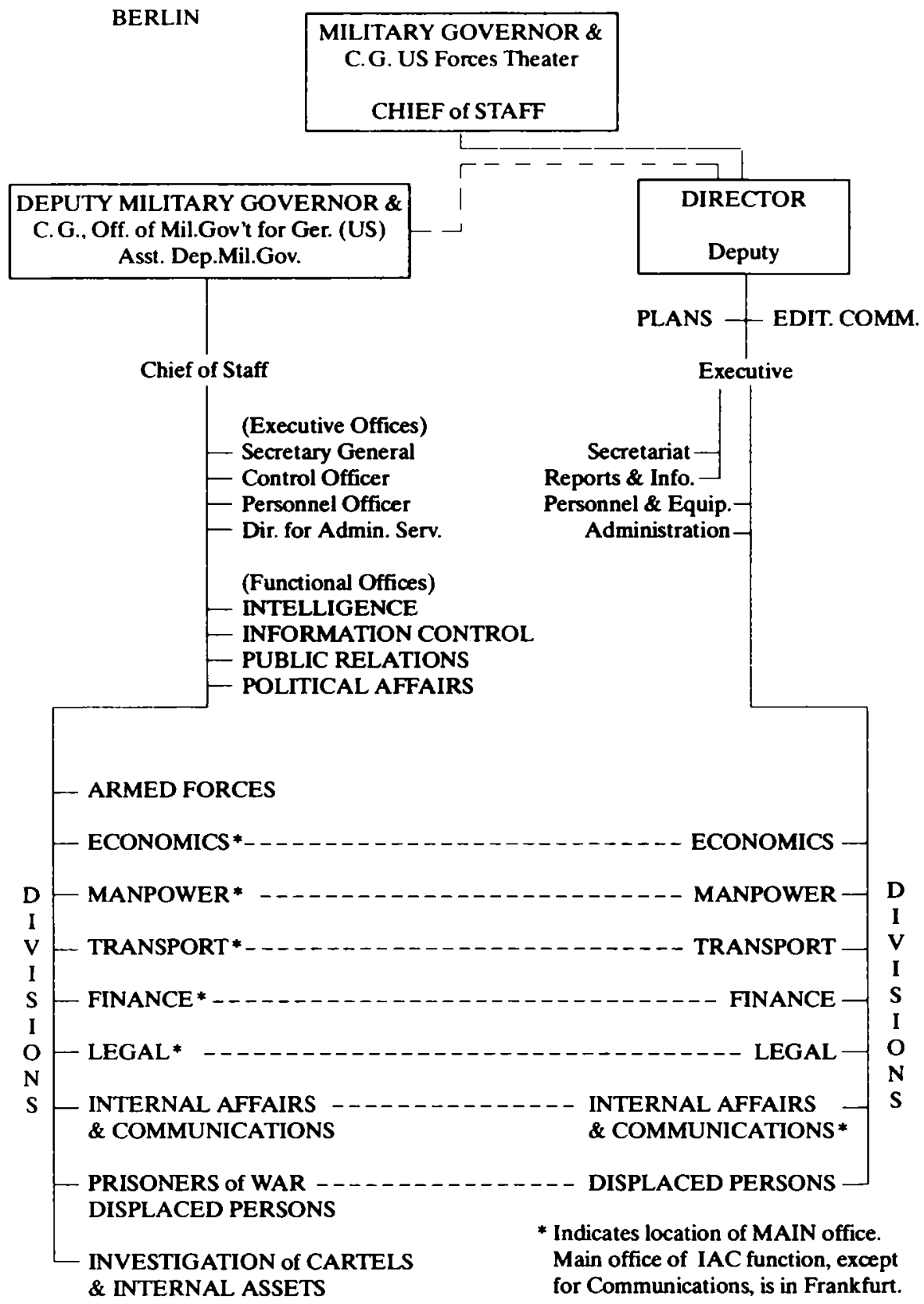

Office of Military Govemment (US ZONE)
BERLIN 
Ubersicht 9: Organisation von OMGUS, Februar 1946

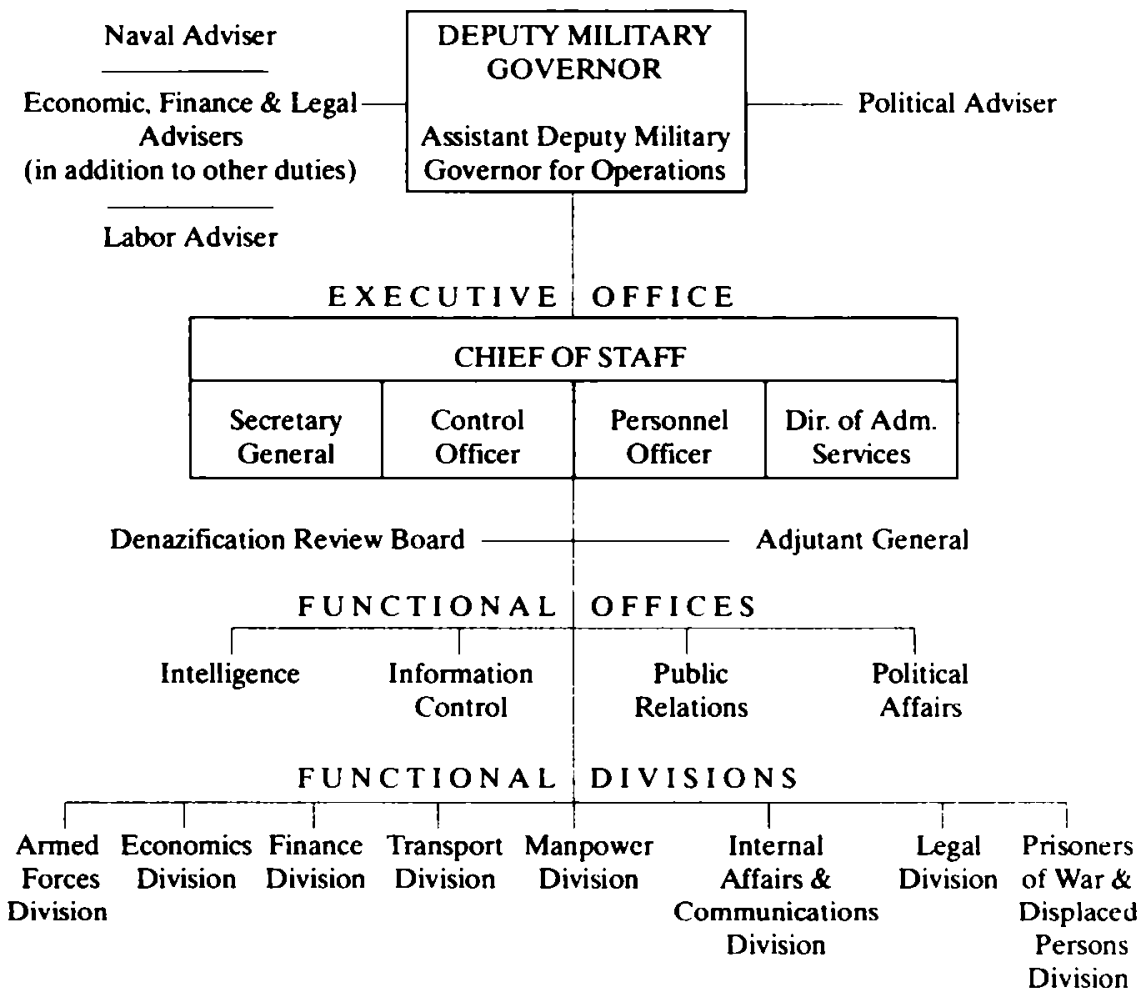

\section{Die Organisation von OMGUS seit April 1946}

Am 7.März 1946 löste USFET das Office of Military Government (US Zone) (OMGUSZ) auf. ${ }^{127}$ Dessen Aufgaben gingen - von geringfügigen Ausnahmen abgesehen ${ }^{128}$ - auf das Office of Military Government for Germany (US) über. Mit Wirkung vom 1. April trat ein Organisations- und Geschäftsverteilungsplan in Kraft, der am 9. bzw. 13. März allen zuständigen Stellen zur Kenntnisnahme und Beachtung übersandt wurde. Das Organization Manual vom 15. März 1946 gab in neun Teilen und mehreren Anlagen einen umfassenden Überblick über den Aufbau der Militärregierung. ${ }^{129}$

In Teil I wurden die Grundsätze der Militärregierung festgelegt, wobei das Prinzip des „indirect rule" gegenüber den deutschen Landesregierungen und Verwaltungsbehörden, die im zentralen Organ des Länderrates für das amerikanische Besatzungsgebiet

127 General Order Nr. 11, OMGUS, vom 13.3.1946 zitiert die General Order Nr.61, USFET, vom 7.3.1946.

128 Die wesentliche Ausnahme stellte die Überwachung der Displaced Persons dar.

129 AG 1945-46/66/2; AG 1945-46/67/6; AG 1945-46/68/9. Die grundsätzliche Bedeutung dieses Organisationshandbuches beweist der Verteiler. Insgesamt wurden 500 Exemplare am 7.3.1946 versandt, davon allein 50 nach Washington und 20 an USFET. 
ihre Spitze fanden, besonders deutlich hervorgehoben wurde. Während Teil II sich mit der Gliederung der Alliierten Kontrollbehörde beschäftigte, beschrieb Teil III Aufgaben und Organisation von OMGUS im einzelnen.

An der Spitze stand das Office of the Deputy Military Governor, also das „Amt“ ${ }^{\text {130 }}$ von General Clay, der gleichzeitig in militärischen Angelegenheiten Kommandierender General (Commanding General) des Office of Military Government for Germany (US) war. Clay wurde durch den Assistant Deputy Military Governor for Operations $^{134}$ in Abwesenheit vertreten und ganz grundsätzlich von Alltagsgeschäften weitgehend entlastet. Dem Ziel der Entlastung des Stellvertretenden Militärgouverneurs diente offensichtlich auch die Erhöhung der Zahl der offiziell institutionalisierten Berater. Neben den bisher schon im Amt befindlichen - Political Adviser, Naval Adviser, Economic Adviser, Legal Adviser und Finance Adviser - trat nun auch ein Labor Adviser, der allerdings im Unterschied zum Wirtschafts-, Rechts- und Finanzberater nicht an der Spitze der korrespondierenden Fachabteilung, der Manpower Division, stand.

Auffällig ist, daß der Begriff Executive Office(s) mehrdeutig verwandt wurde. Unter Executive Office verstand man zum einen ein Kollegialorgan, das aus dem Assistant Deputy Military Governor for Operations, dem Chief of Staff, dem Secretary General, dem Control Officer, dem Personnel Officer, dem Director of Administrative Services und dem Adjutant General ${ }^{132}$ bestand. Dieses Gremium hatte die Arbeit der Fachämter (Functional Offices) und Fachabteilungen (Functional Divisions) sowohl bei der Verwaltung der amerikanischen Zone als auch in der Alliierten Kontrollbehörde nach den Richtlinien des Militärgouverneurs und seines Stellvertreters zu überwachen und zu koordinieren.

Unter einem Executive Office verstand man andererseits jedoch mindestens auch das Amt des Generalsekretärs, das Amt des Kontrolloffiziers, das Amt des Personaloffiziers und das Amt des Direktors für Verwaltungsdienste, die alle unter dem Chef des Stabes zusammengefaßt waren. Weiterhin wurden das Office of Deputy Military Governor, der Denazification Review Board und der Adjutant General in den Quellen auch unter dem Begriff Executive Offices aufgeführt. Der Chef des Stabes war - unterstützt von den vier erstgenannten Exekutivämtern und dem Generaladjutanten befugt, die Arbeit der Fachämter und -abteilungen im Rahmen der ihm von seinen Vorgesetzten gegebenen Richtlinien zu koordinieren und in Einzelfällen, die weder dem Stellvertretenden Militärgouverneur noch seinem Assistant vorgelegt werden mußten, abschließend zu entscheiden.

Das erste der vier unter dem Chef des Stabes zusammengefaßten Executive Offices war das Office of the Secretary General. Der Generalsekretär war für sämtliche Sekretariats- und sonstigen Stabsdienste sowohl für die Militärregierung im amerikanischen Besatzungsgebiet als auch auf Viermächte-Ebene im Kontrollrat zuständig. Über ihn wurden auch die Stabspapiere (Staff Papers) dem Militärgouverneur oder seinem Stellvertreter zur Genehmigung vorgelegt. Außerdem hatte er die Arbeit des Kontrollrats und des Koordinierungssausschusses durch kurze Vermerke für die jeweiligen amerikanischen Vertreter vorzubereiten. Der Generalsekretär war auch dafür verantwortlich, daß die Beschlüsse des Kontrollrats oder seines Koordinierungsausschusses durchgeführt wurden. Zu seiner Unterstützung standen ein Assistant

${ }^{130}$ Der Begriff „Office“ wird im folgenden grundsätzlich mit „Amt“ übersetzt, sofern nicht ausdrücklich etwas anderes vermerkt ist.

131 AG 1945-46/66/2.

132 USGCC 1944-45/10/8, AG 1945-46/68/5. 
Secretary General für Angelegenheiten der amerikanischen Zone und der amerikanische Vertreter im Alliierten Sekretariat des Kontrollrats zur Verfügung.

Der Adjutant General hatte als der Verbindungsoffizier von OMGUS den inneren Verwaltungsablauf von OMGUS zu überwachen und zu beschleunigen. Ihm kam zwar keine ausübende Gewalt zu; er gab aber alle Weisungen von OMGUS heraus, es sei denn, diese wurden wegen ihrer grundsätzlichen Bedeutung oder im Einzelfall vom Chef des Stabes oder einem höheren Vorgesetzten unterzeichnet.

Ein weiteres Executive Office war das Control Office. Seine Funktionen, die sich grundsätzlich mit denen einer Zentralabteilung eines deutschen Ministeriums vergleichen lassen, können nicht besser verdeutlicht werden als durch eine Aufzählung der in ihm zusammengefaßten nächst niedrigeren Verwaltungseinheiten, der Branches:

Ubersicht 10: Organisation des Control Office, OMGUS, April 1946

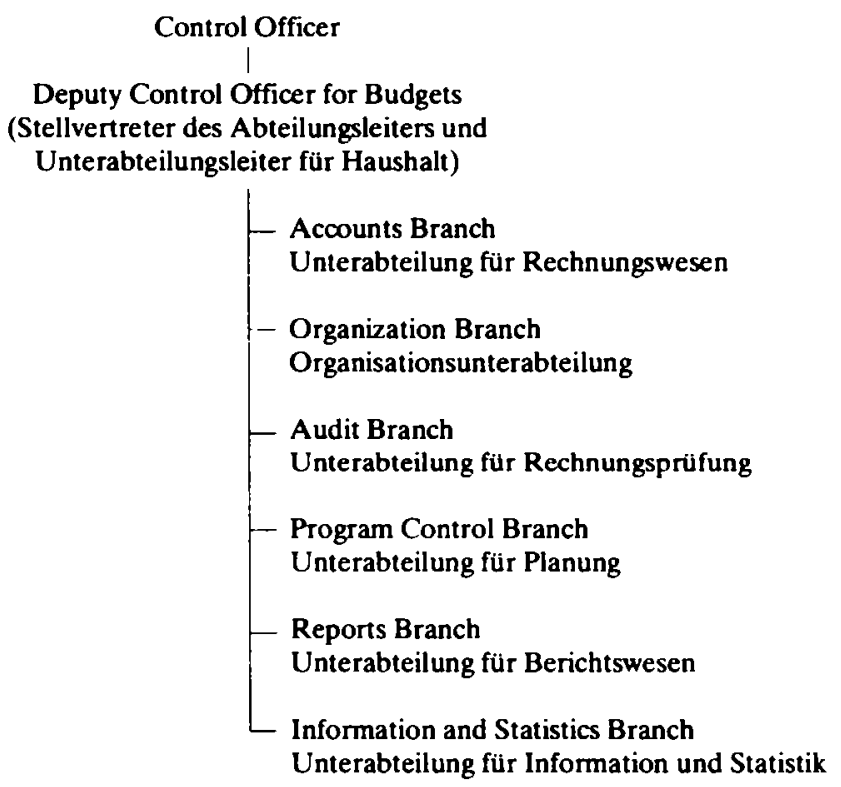

Der Vergleich des Control Office mit einer Zentralabteilung eines Ministeriums hinkt freilich insoweit, als auch der Personnel Officer und der Director for Administrative Services Aufgaben der Dienststellenverwaltung wahrzunehmen hatten. Das Amt des Personaloffiziers gliederte sich in Unterabteilungen für Zivilangestellte, Militärbedienstete, deutsche Angestellte und eine Administrative Branch. Das Amt des Direktors für Verwaltungsdienste bestand aus zwei Teilen, von denen das Headquarters Command in Berlin die Aufgaben des inneren Dienstes auch für den amerikanischen Anteil an der Alliierten Kontrollbehörde und nachgeordnete Stellen in Berlin versah, während der zweite Teil unter dem Sammelbegriff Frankfurt Agencies entsprechende Dienste für den Field Trip Motor Pool und den Kurierdienst im amerikanischen Besatzungsgebiet versah.

Zum Bereich der Executive Offices gehörte auch ein Denazification Review Board. Dessen Vorsitzender hatte das Recht, unmittelbar an den Chef des Stabes zu berichten, besa $\beta$ insoweit eine unabhängigere Stellung. Inhaltlich überprüfte der Denazifica- 
tion Review Board Anträge auf Einstellung oder Wiedereinstellung von ehemaligen Mitgliedern der NSDAP. Angehörige dieses Gremiums waren neben dem Vorsitzenden je ein Vertreter der Internal Affairs and Communications Division, des Director of Political Affairs, der Criminal Investigation Branch, USFET, und derjenigen amerikanischen Fachabteilung, welche die Aufsicht über die deutsche Einrichtung (Behörde oder Unternehmen) führte, bei der das jeweilige ehemalige NSDAP-Mitglied (wieder) eingestellt werden sollte.

Die im Organisationshandbuch enthaltene umfängliche Aufgabenbeschreibung der Leiter der Fachämter und -abteilungen (Functional Offices und Functional Divisions) läßt sich kurz dahingehend zusammenfassen, daß diese die Stellung nicht eines Ministers, sondern eines weisungsgebundenen Staatssekretärs (wie etwa an der Spitze der deutschen Reichsämter unter Bismarck) innehatten. Dieser Vergleich ist jedoch insoweit einzuschränken, als das Executive Office neben Aufgaben der Dienststellenverwaltung in gewisser Weise auch Kabinettfunktionen wahrnahm.

Es gab insgesamt vier Fachämter (Functional Offices). Der Direktor für die Nachrichtendienste (Director of Intelligence) hatte den Stellvertretenden Militärgouverneur mit allen erforderlichen Informationen zu versorgen, die für die amerikanische Militärregierung in Deutschland von Bedeutung sein konnten. Dabei muBte er selbstverständlich enge Verbindung mit seinem entsprechend zuständigen Kollegen bei USFET halten, das als erste Quelle für nachrichtendienstliche Informationen in Europa anzusehen ist. Bei der Informationsbeschaffung hatte er auch mit den Diensten der drei anderen alliierten Mächte zusammenzuarbeiten. Er stand weiterhin an der Spitze des Intelligence Coordinating Committee, in dem außer ihm der Control Officer, der Director of Political Affairs, der Director of Information Control, der Director of Armed Forces Division, der Director of Economics Division und der Leiter der Public Safety Branch in der Division of Internal Affairs and Communications vertreten waren. Außerdem war er für den amerikanischen Anteil im Combined Travel Security Board, einer Viermächteeinrichtung, verantwortlich, die den Reiseverkehr zwischen den vier Zonen in Deutschland überwachte. In Zusammenarbeit mit der Wirtschaftsabteilung hatte der Director of Intelligence schließlich eine Übersicht über die deutschen Wirtschaftsführer zu erstellen, die sich aufgrund enger Verflechtungen mit dem NS-Regime entweder als Kriegsverbrecher vor Gericht verantworten sollten oder als Sicherheitsrisiko gefangen gesetzt werden mußten.

Das zweite Fachamt, das Office of the Director of Information Control (Informationsund Nachrichtenüberwachung), war sowohl als Zensurorgan als auch als Nachrichtensammelstelle im amerikanischen Besatzungsgebiet anzusehen. Es sollte seine Tätigkeit in politischen Angelegenheiten in enger Zusammenarbeit mit dem Political Adviser verrichten und bestand aus einer Staff Group (Stabsunterabteilung für Grundsatzfragen) und den folgenden Fachunterabteilungen (Branches):

Ubersicht 11: Organisation des Office of the Director of Information, OMGUS, April 1946

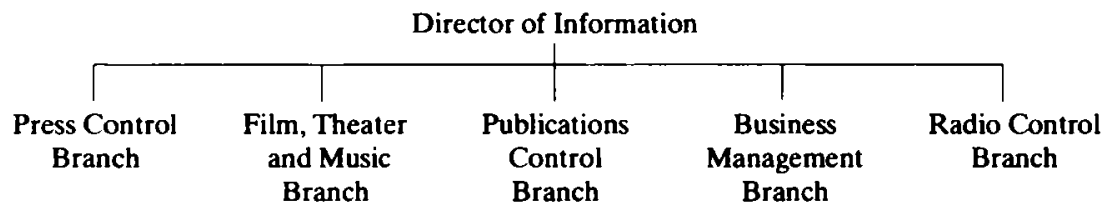

Das dritte Fachamt war das Office of Director of Public Relations, das den amerikanischen Medien ein vollständiges und wahrheitsgemäßes Bild der Tätigkeit der amerika- 
nischen Militärregierung in Deutschland vermitteln sollte. Der Amtsleiter. sein Vertreter und ein weiterer Offizier hatten vor allem auch die Offiziere für die Öffentlichkeitsarbeit in den anderen Fachämtern und -abteilungen der amerikanischen Militärregierung zu unterstützen. Organisatorisch gliederte sich dieses Amt in eine News Branch (Pressewesen) und eine Features Branch, wobei letztere für alle zum Bereich der Information durch Ton (Hörfunk) und Bild gehörenden Zweige der Öffentlichkeitsarbeit und für die Unterrichtung der Bediensteten von OMGUS über Ereignisse in Amerika verantwortlich war.

Das vierte, allerdings keineswegs unbedeutende Fachamt wurde vom Director of Political Affairs geleitet. Seine Mitarbeiter und er waren für alle Probleme mit politischer Relevanz für Deutschland als Ganzes beratend und koordinierend tätig, also für ein sehr umfassendes Aufgabengebiet, das außerhalb einer Besatzungsmacht die vielfältigen Funktionen eines Außenministeriums bzw. einer Botschaft widerspiegeln würde. Dies schloß die Zuständigkeit für Konsulatsangelegenheiten ausdrücklich ein. Es gab insgesamt fünf Unterabteilungen:

\section{Ubersicht 12: Organisation des Director of Political Affairs, OMGUS. April 1946}

Director of Political Affairs

\footnotetext{
- Administrative Branch (Dienstellenverwaltung)

Consular Branch (einschließlich der Aufsicht über die amerikanischen Konsulate in Berlin, Bremen, Frankfurt, Hamburg, München und Stuttgart)

Economic Liaison Branch (Verkehr, Nachrichtenwesen, Finanzen, Ernährung und Landwirtschaft, öffentliche Gesundheitsfürsorge. Industrie, Nachrichtendienste, Rechtswesen, innere Verwaltung, Reparationen und Restitutionen, Statistik, Handel und Kartellangelegenheiten)

Political Research and Analysis Branch (Recherchen und andere - Nachforschungen, auch über den deutschen öffentlichen Dienst. Auswertung und Übersetzung)

Political Branch (Berichtswesen, Archive und Behördenakten, Verschleppte, Kriegsgefangene, Bevölkerungswegung, Erziehung, Kultusangelegenheiten, deutsche Regierungsstruktur, Entnazifizierung, Arbeit und Wohnungsbau)
}

Eine präzise Definition des Unterschieds zwischen einem Fachamt (Functional Office) und einer Fachabteilung (Functional Division) ( $^{133}$ enthält das umfangreiche Organisationshandbuch nicht. Sicher ist jedoch, da $\beta$ in der amerikanischen Verwaltung ein Office grundsätzlich einen höheren Rang hat als eine Division. In diesem Fall dürfte der höhere Rang ausschließlich in der größeren politischen Bedeutung der Ämter liegen und nicht ein Nachordnungsverhältnis der Abteilungen bezeichnen, da die Divisions nicht den Fachämtern, sondern dem Executive Office unmittelbar nachgeordnet waren. Insoweit leuchtet dann auch der Unterschied zwischen dem Executive Office und den Functional Offices ein.

133 „Division“ wird grundsätzlich mit „Abteilung“ übersetzt, sofern im Einzelfall nichts anderes vermerkt ist. 
Es gab im Frühjahr 1946 insgesamt acht Fachabteilungen (Functional Divisions). Die Armed Forces Division war für die Demobilisierung und Entwaffnung der deutschen Streitkräfte und paramilitärischen Organisationen, die Überwachung des deutschen Kriegspotentials, die Ausrottung des deutschen Militarismus und die Behandlung von Kriegsgefangenen ebenso zuständig wie für die Überwachung des Luftverkehrs über Deutschland. Sie hatte somit keine unmittelbare Zuständigkeit für die amerikanischen Truppen: Kompetenzkonflikte mit dem Marineberater waren durch eine saubere Organisationsabgrenzung offensichtlich ausgeschlossen. Die Abteilung gliederte sich in drei Unterabteilungen: Demobilization Branch, Disarmament Branch und Aeronautics Branch.

Die Economics Division bestand aus einem Stab mit Referaten (Sections) für politische Grundsätze, Koordinierung, Berichtswesen, Statistik, Beschaffung und Überwachung deutscher Forschungseinrichtungen sowie sechs Unterabteilungen (Branches):

\section{Ubersicht 13: Organisation der Economics Division, OMGUS, April 1946}

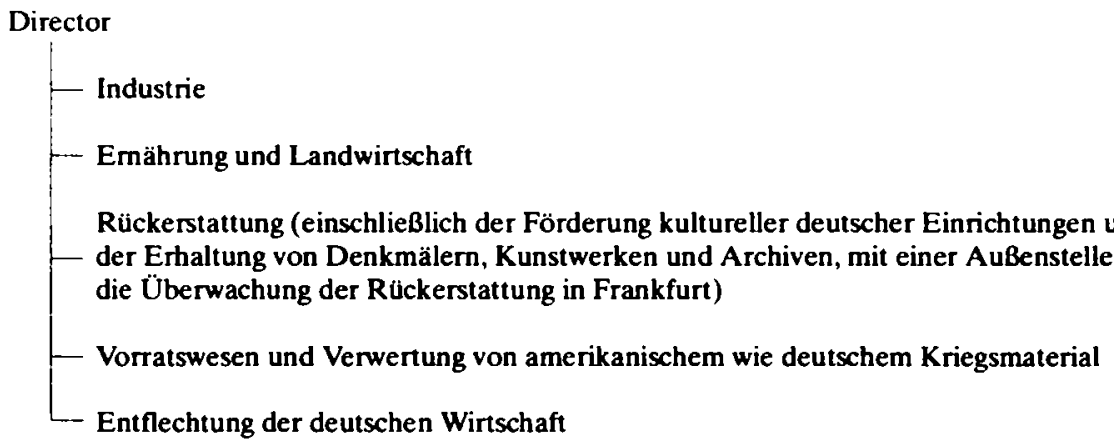

Der Decartelization Branch waren einige Außenstellen mit Spezialaufgaben (z. B. der Überwachung der I. G. Farbenindustrie in Abwicklung) nachgeordnet.

Die Finance Division verfügte über sechs Unterabteilungen für allgemeine Finanzpolitik, Geldinstitute, Devisenbewirtschaftung und eingefrorene Vermögen (einschließlich der Finanzierung von Ein- und Ausfuhren), Vermögensüberwachung, Informationsbeschaffung (Financial Intelligence and Liaison Branch) und für deutsches Auslandsvermögen. Hinzu kam eine Außenstelle (Field Unit) bei der Reichsbankzentrale in Frankfurt am Main.

Die Transport Division umfaßte drei Unterabteilungen für Eisenbahnen, Straßenwesen und Wasserwege mit zahlreichen nachgeordneten Stellen. Hinzu kam ein Movements Office mit einer Zweigstelle in Frankfurt, das den ungehinderten Verkehr der Besatzungsmacht auch außerhalb der eigenen Zone, insbesondere nach Berlin sicherzustellen hatte.

Die Manpower Division setzte sich aus sechs Unterabteilungen mit folgenden Kompetenzen zusammen (Übersicht 14).

Die große und politisch sehr bedeutsame Internal Affairs and Communications Division bestand aus vier Unterabteilungen. Die Unterabteilung für öffentliche Verwaltung (Civil Administration Branch) sollte das politische Ziel der Dezentralisierung der deutschen Verwaltung unter Stärkung der Selbstverwaltung auf lokaler Ebene in die Tat umsetzen bzw. entsprechende deutsche Maßnahmen überwachen. Dabei war auf die Anwendung demokratischer Grundsätze und die Sicherung demokratischer Frei- 
Übersicht 14: Organisation der Manpower Division, OMGUS, April 1946

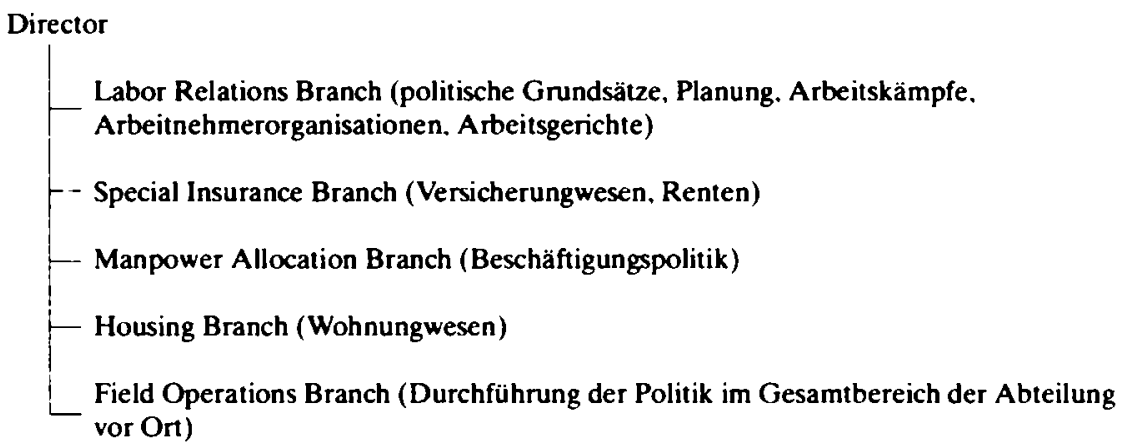

heiten zu achten, die politische Diskussion vor allem zwischen den demokratischen Parteien zu fördern und freie Wahlen zu ermöglichen. Außerdem war diese Unterabteilung für das öffentliche Dienstrecht in der amerikanischen Zone Deutschlands verantwortlich. Während der Zuständigkeitsbereich der Communications Branch mit Post- und Fernmeldewesen kurz zu umschreiben ist, verfügte die Public Health and Welfare Branch über Kompetenzen auf recht unterschiedlichen Gebieten. Zu diesen gehörten nämlich das Schul- und Unterrichtswesen, die Drogenüberwachung sowie die Steuerung der Ernährungslage, die in Deutschland nicht besser sein sollte als im übrigen Europa. Bei dieser Vielzahl von Zuständigkeiten nimmt es nicht wunder, daB das Organisationshandbuch besondere Vorschriften über die Notwendigkeit einer Beteiligung anderer Organisationseinheiten enthielt. Besondere Bedeutung kommt der Public Safety Branch in dieser Abteilung zu. Zum Bereich der öffentlichen Sicherheit zählte man nicht nur wie üblich das Polizei- und Feuerschutzwesen, sondern auch die Überwachung der Deutschen schlechthin, vor allem bei der Entnazifizierung. aber auch bei der Kontrolle des Interzonenverkehrs. Die politischen Gesichtspunkte waren mit dem Director of Political Affairs abzustimmen. Besonderes Gewicht hatte die Aufgabe, die amerikanischen Ansichten über die Entnazifizierung und „Umerziehung" (reeducation) der Deutschen im Kontrollrat zu vertreten.

Die Legal Division, deren Leiter in seiner Funktion als Legal Adviser gleichzeitig Rechtsberater und Justitiar des Militärgouverneurs und dessen Stellvertreters war, gliederte sich in vier Unterabteilungen, denen eine Aufgabe gemeinsam gestellt war: die Säuberung des deutschen Rechts und der deutschen Justiz von nationalsozialistischem Gedankengut. Die Legal Advice Branch beriet OMGUS beim Erlaß von Gesetzen, sonstigen Rechtsvorschriften und grundsätzlichen Weisungen, bei der Überwachung der Gesetzgebung durch den Länderrat und die Länder sowie nicht zuletzt auf dem Gebiet der Prüfung der Rechtsförmlichkeit bei der Rechtsetzung durch amerikanische wie deutsche Stellen. Die Administration of Justice Branch war für die Justizverwaltung und Gerichte im weitesten Sinne zuständig, wobei das Gefängniswesen in einer eigenen Unterabteilung (Prisons Branch) bearbeitet wurde. Die War Crimes Branch war für alle Fragen verantwortlich, die sich nicht nur in der amerikanischen Zone, sondern in ganz Deutschland aus der Notwendigkeit ergaben, wirkliche oder vermeintliche Kriegsverbrecher zu ermitteln und zu bestrafen. Dazu gehörte auch die Auslieferung solcher Personen an Stellen außerhalb Deutschlands oder außerhalb der amerikanischen Zone. Die United Nations War Crimes Commission, das Office of US Chief Counsel for War Crimes, der Theater Judge Advocate und das War Crimes 
Office beim Jugde Advocate General in Washington waren der Unterabteilung zugeordnet.

Die achte Fachabteilung. die Prisoners of War and Displaced Persons Division. war für Fragen der Kriegsgefangenen, der Verschleppten, der Staatenlosen, der Flüchtlinge und Vertriebenen verantwortlich und verrichtete ihre Arbeit in drei Unterabteilungen: Refugees and Prisoners of War Branch, Displaced Persons and Stateless Persons Branch sowie Repatriation Branch.

Auf der Ebene des Kontrollrats hatte diese Abteilung die Vereinigten Staaten in der Combined Repatriation Executive und im Central Tracing Board zu vertreten, die beide unter dem Prisoners of War and Displaced Persons Directorate, ACA, für diese Personengruppen verantwortlich waren. Aus organisatorischer Sicht mag die Arbeit der Abteilung vor allem dadurch erschwert erscheinen, daB innerhalb G-5, USFET, eine Displaced Persons Branch über Kompetenzen verfügte, die sich praktisch mit den Zuständigkeiten von OMGUS überschnitten. Daher schrieb eine USFET-Weisung vom 7. März 1946 aus guten Gründen vor, daß der Leiter der OMGUS-Abteilung für Kriegsgefangene und Verschleppte gleichzeitig an der Spitze der entsprechenden Unterabteilung von G-5, USFET, stand. Bei dieser Gelegenheit ist anzumerken, daB auch andere Stellen von OMGUS Verbindungsoffiziere bei USFET in Frankfurt unterhielten.

Auf den ersten Blick überraschend erscheint, daß das Regional Government Coordinating Office (RGCO) in Teil IV des Handbuches unter Special Agencies and Field Units aufgeführt wird. Diese Zuordnung hat formal ihre Berechtigung, weil diese Behörde nicht in Berlin, sondern in Stuttgart als Koordinierungsorgan zwischen der amerikanischen Militärregierung und dem deutschen Länderrat tätig war. Politisch gehörte das Regional Government Coordinating Office jedoch mit Sicherheit zu den wichtigsten zentralen Verwaltungseinheiten der Militärregierung, weil sein Leiter unmittelbares Vortragsrecht beim Deputy Military Governor hatte und alle Weisungen oder Verlautbarungen der Militärregierung an den Länderrat über diese Koordinierungsstelle laufen muBten.

Von den nachgeordneten Dienststellen im engeren Sinne war die Field Information Agency, Technical (FIAT) eine der wichtigsten. Sie verwertete technologisch relevante Erkenntnisse der deutschen Forschung und Industrie für Zwecke der Vereinigten Staaten. Die Vielfalt ihrer Tätigkeit ergibt sich bereits aus der Gliederung in vier Ämter und sechs Unterabteilungen. Die Ämter waren für die Verbindung mit den Engländern und Franzosen, für Berliner Angelegenheiten, für allgemeine Planungsfragen sowie für die Dienststellenverwaltung verantwortlich. Die Unterabteilungen trugen die Bezeichnungen Operations Branch, Documents Branch, Publications Branch, Scientific Branch (Physik, Chemie, Biochemie und Biologie), Records Branch (mit Zuständigkeiten auch für Statistik und Bibliotheksangelegenheiten) sowie Industry Branch. Der Leiter von FIAT hatte unmittelbares Vortragsrecht beim Chef des Stabes von OMGUS.

Der Combined Travel Security Board und die ihm nachgeordneten Military Permit Offices waren als gemeinsame Einrichtungen der drei Westalliierten für die Kontrolle und Sicherung des interzonalen und internationalen Reiseverkehrs zuständig. Für OMGUS ubte der Director of Intelligence die Fachaufsicht aus. Schließlich sind ein Military Government Courier Service und ein Field Trip Motor Pool, beide in Frankfurt, zu nennen, die beide dem Director of Administrative Services nachgeordnet waren. Aus naheliegenden Gründen unterhielt die Militärregierung ferner eigene nachgeordnete Behörden (Field Offices) für die Überwachung der deutschen Wasser- und Schiffahrtswege sowie der Eisenbahnverwaltung. Das Headquarters Command war 
im weitesten Sinne die militärische Dienststellenverwaltung für OMGUS und die amerikanischen Truppen in Berlin.

Das Berlin Document Center und die mit ihm verbundenen Spezialeinrichtungen des Ministerial Collecting Center und der US Microfilm Organization waren für die Bereitstellung und Auswertung der Akten der NSDAP, ihrer Gliederungen und angeschlossenen Verbände sowie der deutschen Ministerialakten und für die Herstellung von Mikrofilmreproduktionen für Zwecke der Militärregierung verantwortlich. Vorgesetzte Stelle bei OMGUS war der Director of Administrative Services.

Unmittelbar nach Herstellung des Organisationshandbuches erging noch im April 1946 die Verordnung Nr. 2 „Gerichte der Militärregierung“, nach der eine dreistufige Militärgerichtsbarkeit aufzubauen war, um die „Aburteilung von Straftaten gegen die Belange der Alliierten Streitkräfte“ zu ermöglichen. Unter den Oberen Militärgerichten (General Military Courts) verrichteten Mittlere Militärgerichte (Intermediate Military Courts) und Einfache Militärgerichte (Summary Military Courts) ihren Dienst. ${ }^{134}$ Durch Verordnung Nr. 6 vom 21. Mai 1946 wurde dann ein Zivilgericht der Militärregierung (Military Government Court for Civil Actions) für solche Zivilsachen errichtet, an denen mindestens ein amerikanischer Staatsbürger oder ein Bürger eines anderen Staates der Vereinten Nationen beteiligt war. ${ }^{135}$

\section{Instanzenzüge und Befehlswege innerhalb der amerikanischen Besatzungsverwaltung}

Grundlegende Instruktionen wurden als Military Government Regulations (MGR) in einer sachsystematischen Sammlung - nach Titeln und Abschnitten organisiert - in gedruckter Form herausgegeben. ${ }^{136}$ In dringenden Fällen oder bei besonderen Anlässen waren natürlich Ausnahmen von diesem Verfahren zugelassen. Bei militärischen Befehlen (Directives, Orders) lief der Instanzenzug entweder vom Theater Commander zum Commanding General von OMGUS, zum Commanding General für den amerikanischen Sektor von Berlin, zum Commanding General des Bremen Port Command oder vom Commanding General von OMGUS zu den Militärregierungen der Länder oder anderen unmittelbar nachgeordneten Dienststellen. Besondere Formvorschriften waren dabei nicht zu beachten. Inhaltlich wurden grundlegende Weisungen durch Stabsstudien (Staff Studies) vorbereitet.

Im zivilen Bereich gab es Executive Orders oder Instructions an die Militärregierungen in München, Stuttgart, Wiesbaden, Bremen und Berlin (Berlin Sector). Solche Weisungen ergingen vom Executive Office in Berlin an die entsprechenden nachgeordneten Stellen. Davon zu unterscheiden sind Functional Instructions, die von jeder Stelle, die eine Fachaufsicht ausübte, unmittelbar an die nachgeordnete fachlich entsprechend verantwortliche Verwaltungseinheit (z. B. Economics Division, OMGUS, an die Economics Division, Office of Military Government for Bavaria) ergingen.

${ }^{134}$ Military Government Gazette, Germany. United States Area of Control, S. A 60 ff.

135 Ebenda, S. A $73 \mathrm{ff}$.

136 Military Government Regulations [MGR], Title 1 General Provisions; Title 2 Eradication of Nazism and Militarism; Title 3 Political Activities; Title 4 Civil Administration .. . Title $23 \mathrm{Mi}$ litary Government Legislation; Title 24 Important German Legislation usw. Die Titles wurden in Parts (Teile), z. B. 1-400 Military Government Organization and Functions, die Teile in Sections (Sektionen), z. B. 1-405 Control Council gegliedert. Vgl. Military Government Weekly Bulletin Nr. 78 vom 3.2.1947. 
Die Verbindung zwischen USFET und OMGUS lief grundsätzlich über den Assistant Chief of Staff und G-5, USFET: die Armed Forces Division, der Naval Adviser. der Director of Public Relations und der Director of Intelligence, alle bei OMGUS. brauchten dagegen den Dienstweg über G-5, USFET, nicht einzuhalten. Auch der Personnel Officer, der Control Officer und einige andere Stellen hatten für bestimmte Bereiche das Recht auf unmittelbaren Schriftverkehr mit OMGUS.

Diese Instanzenzüge wurden dann verwässert, wenn Sonderberater Clays für bestimmte Aufgaben ernannt wurden. So wurde z. B. bereits durch General Order Nr. 17 vom 9. April 1946 Walter L. Dorn zum Sonderberater des Deputy Military Governor für die Beziehungen zwischen den Military Government Detachments und den deutschen regionalen Dienststellen ernannt. ${ }^{137}$ Eine entsprechende Funktion erhielt Dorn kurze Zeit später für Fragen der Entnazifizierung. ${ }^{1.38}$

Die Neigung, besondere Probleme Sonderbeauftragten zuzuweisen, fand schließlich ihren Ausdruck in der Übertragung der Zuständigkeit von Grundsatzfragen der Reisekontrolle auf den Adjutant General. Auf der anderen Seite war die Einrichtung abteilungsübergreifender Boards ein Mittel der Straffung und Koordinierung der Verwaltung.

\section{Die Gründung der Civil Administration Division}

Durch General Order Nr.24 vom 26. April 1946 wurde die Civil Administration Branch in der Internal Affairs and Communications Division in eine Civil Administration Division umgewandelt und Henry Parkman mit sofortiger Wirkung zum Abteilungsleiter ernannt. ${ }^{139}$ Dabei änderte sich der Zuständigkeitsbereich der Civil Administration Division gegenüber der früheren Civil Administration Branch im Grundsatz nicht. Sie blieb verantwortlich für die künftige deutsche Regierungsorganisation, Wahlen, politische Parteien und die Überwachung der nachgeordneten Stellen der Militärregierung. Hinzugefügt waren von Anfang an Kompetenzen auf dem Gebiet der Bevölkerungsentwicklung und -statistik. Die neue Abteilung, deren Errichtung eine Änderung der sachlichen Prioritäten der Militärregierung bedeutete, gliederte sich in sechs Unterabteilungen (Übersicht 15).

Eine weitere bedeutsame Änderung im Bereich der inneren Verwaltung trat durch General Order Nr. 40 vom 10. Juni 1946 mit Wirkung vom 1.Juni 1946 in Kraft. Das Office of Personal Adviser to the Deputy Military Governor on Denazification wurde errichtet. Zum Personal Adviser on Denazification wurde Walter L. Dorn unter Beibehaltung seiner bisherigen Pflichten ernannt. Dadurch kam die besondere Bedeutung, die Clay und seine Mitarbeiter der Entnazifizierung beimaßen, in hervorragender Weise zum Ausdruck; die Errichtung dieser Sonderdienststelle bedeutete allerdings offensichtlich auch eine Kritik an dem bisherigen Entnazifizierungsverfahren. Die Aufgabe Dorns bestand nicht nur in der Beratung Clays, sondern auch in der Teilnahme an den Sitzungen des Entnazifizierungsausschusses des Länderrates, in der Unterstützung der deutschen Minister für politische Befreiung bei der Durchführung der Entnazifizierungsgesetze und in der Übernahme des Vorsitzes in dem Military Government Denazification Coordinating Committee, das ebenfalls Anfang Juni

${ }^{137} \mathrm{Vgl}$. Dorn: Inspektionsreisen in der US-Zone.

${ }^{138}$ General Order Nr.61 vom 15.10 .1946$.

139 General Order Nr. 24 vom 26.4.1946; 5/363-1/19. 
Übersicht 15: Organisation der Civil Administration Division, OMGUS. April 1946

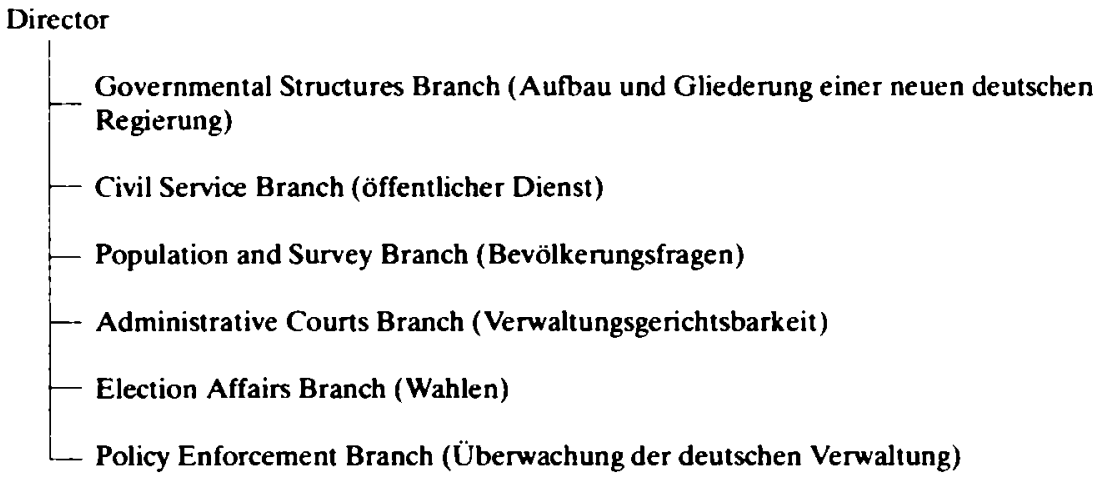

errichtet wurde. Die sachliche Zuständigkeit des Leiters der Unterabteilung für öffentliche Sicherheit in der Internal Affairs and Communications Division blieb grundsätzlich unberührt. Er war jetzt lediglich gehalten, Verfahrens- und Durchführungsvorschriften nach der Gesetzgebung über die Befreiung vom Nationalsozialismus und Militarismus dem neu ernannten Koordinierungsausschuß zur Genehmigung vorzulegen. Mitglieder dieses Ausschusses waren außer Dorn und dem Leiter der Public Safety Branch, der den Sonderberater im Verhinderungsfall zu vertreten hatte. der Leiter des Referats für Entnazifizierung in der Public Safety Branch, die zuständigen Offiziere bei den Landesmilitärregierungen, der Political Adviser und der Denazification Legal Officer. Diese Stelle wurde damals ebenfalls neu geschaffen und Philip Elman aus der Rechtsabteilung von OMGUS unter Beibehaltung seiner übrigen Pflichten übertragen.

Übersicht 16: Organisation und Personal von OMGUS, Dezember 1946

\begin{tabular}{|c|c|c|}
\hline Military Governor & Gen. & Joseph T.McNarney \\
\hline Deputy Military Governor & Lt.Gen. & Lucius D.Clay \\
\hline $\begin{array}{l}\text { Special Adviser to Deputy Military Governor for } \\
\text { German Civil Administration }\end{array}$ & Civ. & Walter L. Dorn \\
\hline Deputy Military Governor for Operations & Maj.Gen. & Frank A. Keating \\
\hline Chief of Staff & Brig.Gen. & Charles K. Gailey \\
\hline Secretary General & Col. & William Whipple \\
\hline Adjutant General & Lt.Col. & George H.Garde \\
\hline \multicolumn{3}{|l|}{ Armed Forces Division } \\
\hline Director & Maj.Gen. & Robert W.Harper \\
\hline Demilitarization Branch, Chief & Col. & W. W. Holler \\
\hline Aviation Branch, Chief & Civ. & G.M. Jones \\
\hline Personnel and Services Branch, Chief & Col. & L. E. Hobbs \\
\hline \multicolumn{3}{|l|}{ Civil Administration Division } \\
\hline Director & Civ. & Henry Parkman \\
\hline Deputy Director & Civ. & Edward H. Litchfield \\
\hline Govemmental Structures Branch, Chief & Civ. & Kenneth A. Dayton \\
\hline Civil Service Branch, Chief & Lt.Col. & R.P. Rosengren \\
\hline Population and Survey Branch, Chief & Civ. & Karl A. Shafer \\
\hline Administrative Courts Branch, Chief & Civ. & B.D.Sartin \\
\hline
\end{tabular}


Election Affairs Branch. Chief

Policy Enforcement Branch. Chief

Control Office. Control Officer

Assistant Control Officer for Management

Assistant Control Officer for Statistical Standards

Budget Branch. Chief

Accounts Branch, Acting Chief

Organization Branch. Chief

Reports Branch, Acting Chief

Program Branch, Chief

Statistics Branch. Chief
Civ.
Col.
Col.
Civ.
Civ.
Civ.
Civ.
Civ.
Civ.
Civ.
Civ.

Brig.Gen.

Civ.

Civ.

Col.

Lt.Col.

Civ.

Civ.

Civ.

Civ.

Col.

Civ.

Civ.

Civ.

Col.

Col.

Civ.

Lt.Col.

Civ.

Civ.

Civ.

Civ.

Civ.

Civ.

Civ.

Col.

Brig.Gen. Lt.Col.

Civ.

Civ.

Maj.

Civ.

Lt.Col.

Civ.

Civ.

Civ.

Civ.
R.M. Scammon

M. Boyle

David L. Robinson

Waldemar Thorson

P. M. Rouzitsky

Ralph D.Snow

C.Hall

W.H.Schroder

John T. Butterwick

Milton E. Muelder

Bumham P. Beckwith

William H. Draper

Don D. Humphrey

Peter V.Martin

Stephen A.Park

Thurman S. Doman

Saul Nelson

Malcolm S. McComb

George N. McClusky

George Scatchard

Lawrence Wilkinson

Menc S.Szymczak

James S. Martin

Moore C.McIntosh

Hugh B. Hester

John H. Allen

Malcom S. McComb

Samuel L. Williams

Jack Bennett

Theodore H. Ball

Robert P.March

E.N.Reinsel

Albert Bender

Jo Fisher Freeman

Walter Lichtenstein

William G. Brey

Robert A.McClure

William Kinard

Alfred V. Boemer

Paul Conant

Duncan MacBryde

John Kidder

Douglas Waples

Charles S. Lewis

Robert C. Schmid

Arthur D. Eggleston

Eric T.Clarke 


\section{Ofince of Director of Intelligence}

Director

Analysis, Research and Reports Branch. Chief Liaison Officer

Counterintelligence Policy and Liaison Branch, Chief Civil Censorship Policy and Liaison Branch. Chief

\section{Internal Afiairs and Communications Division}

Acting Director

Deputy Director

Communications Branch. Chief

Public Health and Welfare Branch, Chief

Public Safety Branch. Chief

Education and Religious Affairs Branch, Chief

\section{Legol Division}

Director

Assistant Director

War Crimes Branch, Acting Chief

Administration of Justice Branch. Chief

Legislative Branch, Chief

Prison Branch, Chief

Legal Advice Branch, Chief

Board of Clemency, Chairman

Military Government Court for Civil Actions

(Stuttgart), Judge

\section{Manpower Division}

Director

Reports and Statistical Branch, Chief

Labor Relations Branch. Chief

Wages and Labor Standards Branch. Chief

Social Insurance Branch, Chief

Manpower Allocation Branch, Chief

\section{Ofince of the Naval Adviser}

Naval Adviser

Deputy Naval Adviser

\section{Oficice of the Personnel Ofificer}

Personnel Officer

Military Personnel Branch, Chief

US Civilian Personnel Branch, Chief

Administrative Branch

Indigenous Personnel Branch, Chief

\section{Oiice of the US Political Adviser}

Political Adviser

Director of Political Affairs

Deputy Director of Political Affairs

Information Control Branch, Chief

Economic Branch, Chief

Exploitation German Archives Branch, Chief

Political Branch, Chief

\section{Prisoners of War and Displaced Persons Division}

Director

Displaced Persons and Stateless Persons Branch, Chief
Col

Civ.

Lt.Col.

Maj.

Civ.

Civ.

Col.

Civ.

Col.

Civ.

Civ.

Civ.

Col.

Civ.

Civ.

Civ.

Civ.

Civ.

Civ.

Civ.

Civ.

Civ.

Maj.

Civ.

Civ.

Civ.

Rear Adm.

Capt.

Col.

Col.

Civ.

Lt.Col.

Lt.Col.

Amb.

Civ.

Civ.

\section{R. E. Schuirman}

E. J. Burke

Leo R. Werts

E.L. Deuss

Harold F. Mullaney

Sol D. Ozer

M.G. Murray

George S. Whecler

James T. Duke

Louis G. Gibney

G. B. Robbins

Saul S. Dorfman

Everett A. Luckenbach

Robert D. Murphy

Donald R. Heath

Loyd V. Steere

Thomas J. B. Wenner

Loyd V.Steere

C. P. English

Warren M. Chase

Brig.Gen. Stanley R.Mickelsen

Lt.Col. James P. Abbott 
Refugees and United Nations Prisoners of War Branch. Chief

Repatriation Branch. Chicf

Public Relations Ofrice

Director

\section{Transport Division}

Acting Director

Deputy Director

Road Branch. Chief

Water Branch, Chief

Rail Branch. Chief

Movement and Traffic Branch (Rear Echelon Frankfurt), Chief

$\begin{array}{ll}\text { Lt.Col. } & \text { Charles E. Boyle } \\ \text { Maj. } & \text { John F. Asselta } \\ \text { Civ. } & \text { William R. Haggard } \\ & \\ \text { Col. } & \text { Hans W. Holmer } \\ \text { Col. } & \text { John S. Niles } \\ \text { Civ. } & \text { C.E. Calvert } \\ \text { Civ. } & \text { D.R.Neff } \\ \text { Civ. } & \text { E. V.Breitenbach } \\ \text { Col. } & \text { A.H.Schroeder }\end{array}$

Col.

William W. Dawson

Regional Govemment Coordinating Office (Stuttgart) Director

Otice of the Chief of Counsel for War Crimes (Nümberg) Chief Brig.Gen. Telford C. Taylor

\section{Die Besatzungsverwaltung im Vereinigten Wirtschaftsgebiet ${ }^{140}$}

Die Aufteilung Deutschlands westlich von Oder und Neiße in vier Besatzungszonen spaltete das Land auch wirtschaftlich. In Vorbereitung der Teilnahme der westlichen Zonen am Marshall-Plan war es aus der Sicht vor allem der amerikanischen Besatzungsmacht wichtig, die Nachteile auszugleichen, die dadurch entstanden waren, da $B$ deutsche Zentralbehörden auch für die Bereiche nicht errichtet worden waren, in denen das Potdamer Abkommen vom 2. August 1945 entsprechendes vorsah. Dahingehende amerikanische Vorstöße waren im Kontrollrat immer wieder gescheitert. Die Errichtung eines Länderrates für das amerikanische Besatzungsgebiet mit Sitz in Stuttgart diente dann als erster Schritt zur Verwirklichung der amerikanischen Bestrebungen wenigstens für die eigene Zone.

Obwohl es im Sommer 1946 ganz offensichtlich war, daß keinerlei Aussichten für eine Einigung der vier Mächte im Sinne einer Errichtung deutscher Zentralbehörden für alle vier Zonen bestanden, machte Eisenhowers Nachfolger General Joseph T. McNarney in der 34. Sitzung des Alliierten Kontrollrats am 20. Juli 1946 seitens der amerikanischen Regierung einen offiziellen Vorstoß zur wirtschaftlichen Vereinigung der vier Zonen. In der nächsten Kontrollratssitzung vom 30. Juli 1946 griff jedoch lediglich Großbritannien den amerikanischen Vorschlag auf. Seitens der Sowjetunion und Frankreichs wurde zwar nicht förmlich gegen das amerikanisch-britische Vorgehen protestiert, die Fusion von Verwaltungen aller vier Zonen jedoch de facto abgelehnt.

Am 9. August 1946 trafen sich die beiden Stellvertretenden Militärgouverneure des amerikanischen und britischen Besatzungsgebiets zu den ersten vorbereitenden Gesprächen über die wirtschaftliche Vereinigung ihrer beiden Zonen. Bereits bei diesem Treffen kam man überein, daß die beiden Zonenbefehlshaber gemeinsam an der Spitze einer Bipartite Organization ${ }^{141}$ stehen und sich zu einem Bipartite Board zusam-

140 Benz: Von der Besatzungsherrschaft, S. 35; Benz: Vorformen, S. $375 \mathrm{ff}$.

$141 \mathrm{Vgl}$. zusammenfassend "The Evolution of Bizonal Organization, prepared by Civil Administration Division, OMGUS“, 2. Auflage, August 1948 (BA ZD 110/4); Military Government Weekly Information Bulletin vom 24.3.1947 und 1.9.1947. 


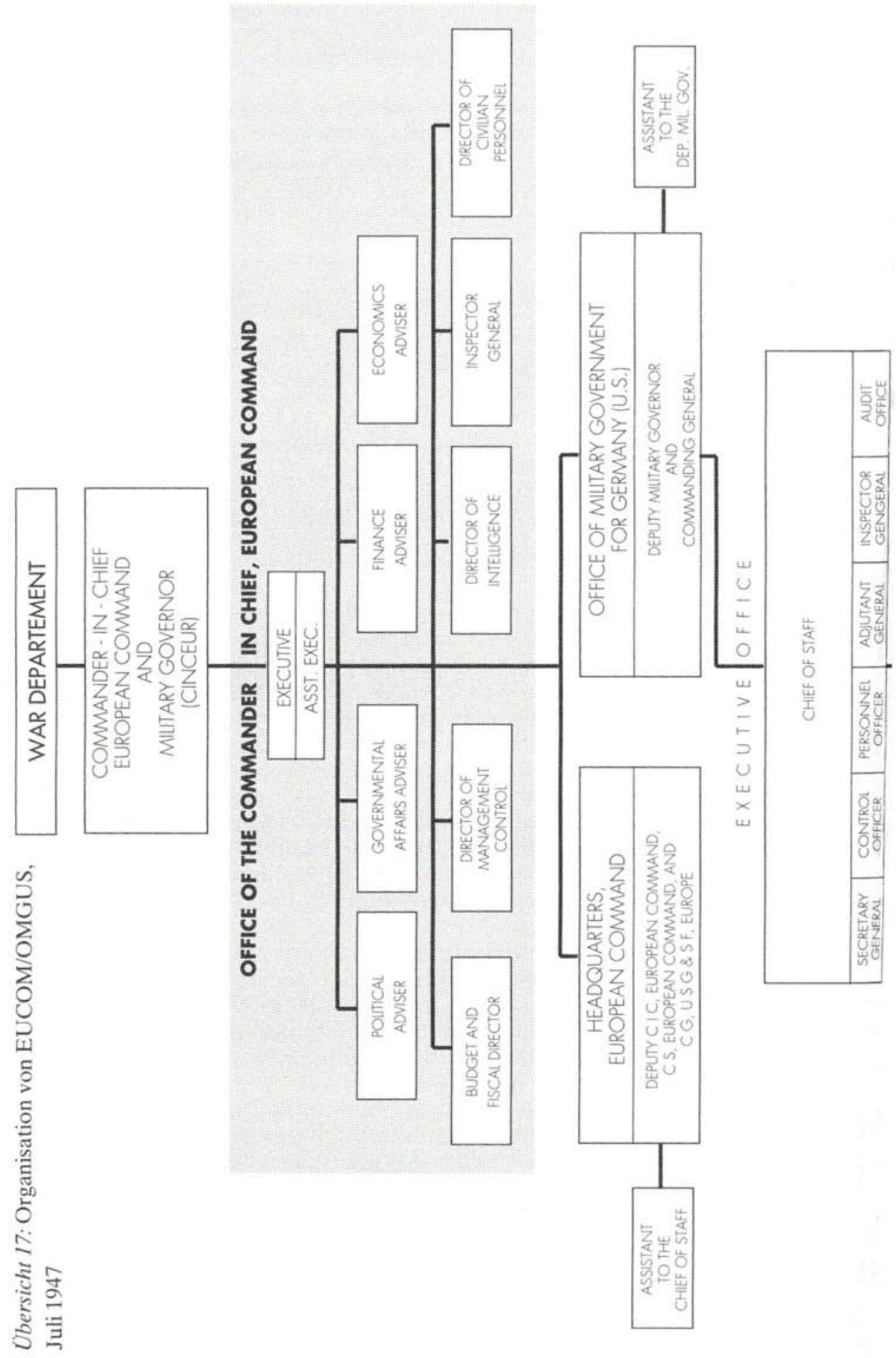




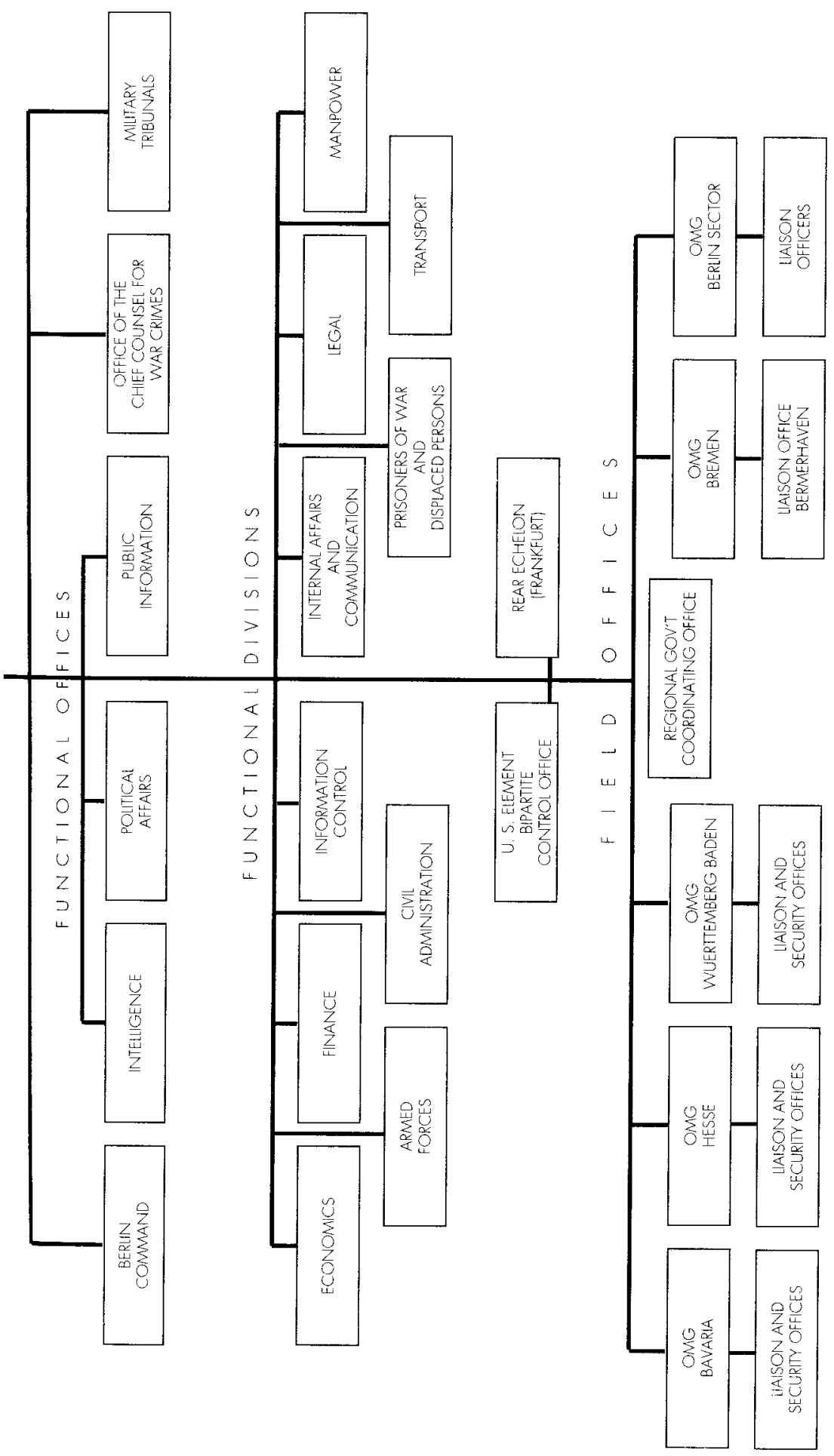


menschließen sollten. Der Gedanke, den Bipartite Board im Rahmen der Viermächtekontrollbehörde zu etablieren, ist offensichtlich erwogen, aber schon bald verworfen worden. Immerhin nannte man den Bipartite Board umgangssprachlich einen Zweimächte-Kontrollrat. ${ }^{142}$ Bei diesem Treffen kam man ferner überein, durch die beiden Stellvertretenden Militärgouverneure Einzelheiten einer gemeinsamen Wirtschaftsorganisation der beiden Zonen ausarbeiten zu lassen. Eine politische oder verwaltungsmäBige Angleichung der Verhältnisse in den beiden Zonen war indes nicht beabsichtigt.

Dem Bipartite Board wurde ein von der Viermächtekontrollverwaltung der Kontrollbehörde ebenfalls völlig unabhängiges Bipartite Secretariat zugeordnet. Wie in Potsdam für alle vier Zonen vorgesehen, wurden nun in der Bizone deutsche Verwaltungsräte (Bizonal Executive Joint Committees) für folgende Sachgebiete gebildet: Wirtschaft, Ernährung und Landwirtschaft, Verkehr, Finanzen sowie Post- und Fernmeldewesen.$^{143}$ In diesen Verwaltungsräten erhielt jedes deutsche Land in den beiden Zonen je einen Sitz, im Frühjahr 1947 auch die beiden Stadtstaaten Bremen und Hamburg. Jedem Verwaltungsrat stand ein eigenes Verwaltungsamt zur Verfügung. das der Vorsitzende des Rates leitete. Diese fünf Verwaltungsämter wurden in fünf Städten errichtet: für Wirtschaft in Minden, für Ernährung und Landwirtschaft in Stuttgart, für Verkehr in Bielefeld, für Finanzen in Bad Homburg und für das Post- und Fernmeldewesen in Frankfurt. In diesen Ämtern sollten die Zentralverwaltungen in der britischen Zone sowie die Ausschüsse und anderen Organe des Länderrats in Stuttgart aufgehen. Die Durchführung der Beschlüsse der Verwaltungsräte war von entsprechenden Gesetzen und Verordnungen der Länder abhängig. Eine unmittelbare Rechtsetzungsbefugnis bestand also nicht. Lehnten ein oder mehrere Landtage eine bizonale Gesetzesvorlage ab, entschieden die Alliierten, ob die Vorlage nur in den Ländern, in denen die Parlamente zugestimmt hatten, Rechtskraft erhielt oder ob sie als Militärregierungsgesetz auch in den anderen Ländern verbindlich gemacht werden sollte. Der Begriff „.bipartite“ wurde offiziell ausschließlich für alliierte, der Begriff „bizonal" nur für deutsche Einrichtungen verwandt.

Die deutschen Verwaltungsräte wurden durch die Militärregierung lückenlos und in mehreren Stufen beaufsichtigt. Ebenso wie der Bipartite Board und das Bipartite Secretariat hatte auch die mittlere Kontrollinstanz ihren Sitz in Berlin. Zunächst Committees genannt, wurden in Berlin britische und amerikanische Fachbeamte auf der Ebene der Abteilungsleiter zu Bipartite Panels zusammengefaßt. Den fünf Fachbereichen entsprechend gab es diese Ausschüsse auf den Gebieten der Wirtschaft, der Finanzen, der Ernährung und Landwirtschaft, des Verkehrs und der Post. Die untere Instanz der Zweimächteverwaltung bildeten Bipartite Control Groups, die ihren Sitz in den Städten hatten, in denen sich die entsprechenden deutschen Verwaltungen befanden. Die Entscheidung der beiden Oberbefehlshaber, die amerikanische und britische Zone zu einer Wirtschaftseinheit zusammenzufassen, wurde formal durch ein Übereinkommen der beiden Außenminister Byrnes und Bevin vom 2. Dezember 1946 bestätigt.

Der zweite Schritt, der eine wesentliche Verstärkung der gemeinsamen britisch-amerikanischen Organisation in der Bizone brachte, erfolgte durch Übereinkunft der beiden Mächte vom 29. Mai 1947. Damals wurden die deutschen Verwaltungsräte in Ver-

142 Eckhardt: Ein unordentliches Leben, S. 139. Vgl. Report of the Military Governor, Nr. 14 vom 20.9.1946, S.1.

$143 \mathrm{Zu}$ den Einzelheiten des Verwaltungsaufbaus vgl. vor allem Vogel: Westdeutschland 1-3. 
waltungen (Departments) umgewandelt sowie ein deutscher Wirtschaftsrat und ein Exekutivrat errichtet. dem Vertreter aller acht Länder des Vereinigten Wirtschaftsgebietes angehörten. Auf alliierter Seite blieben Bipartite Board und Bipartite Secretariat in Berlin bestehen, doch wurden die bisherigen Bipartite Panels in Berlin und die bei den bisherigen deutschen Verwaltungsämtern eingerichteten Bipartatite Control Groups aufgelöst. Allerdings berieten bisherige Mitglieder der Panels in Berlin auch in Zukunft die Leiter der Fachabteilungen von OMGUS bzw. britischer Militärregierung. In Frankfurt am Main wurde ein Bipartite Control Office (Zweizonen-Kontrollamt) errichtet. BICO - so lautete die bald gebräuchliche Kurzform - hatte je einen britischen und amerikanischen Leiter. Im Auftrag der beiden Militärgouverneure übte BICO die Kontrolle über den Exekutivrat und die nunmehr von Direktoren geleiteten fünf deutschen Verwaltungen aus. BICO mit seinen etwa 900 Mitarbeitern ist weniger als Behörde, sondern vielmehr als Koordinierungsorgan der beiden Besatzungsmächte anzusehen. Die Federführung wechselte zwischen dem amerikanischen (US Element) und dem britischen (British Element) Anteil, an deren Spitze ein Office of the Chairman - jeweils eines für jede Besatzungsmacht - und sechs Unterabteilungen (Bipartite Groups) stand. Die Verlagerung sowohl der alliierten als auch der deutschen Behörden nach Frankfurt erfolgte nur schrittweise.

Seit dem Herbst 1947 wurde die Arbeit des Bipartite Board, also der beiden Militärgouverneure oder ihrer Stellvertreter, nicht nur vom Sekretariat, sondern auch von mehreren Beratern (Advisers) unterstützt, die meist den früheren Panels angehört hatten. Das Bipartite Control Office bestand damals - sieht man von dem Office of the Chairman $a b$, in dem ein amerikanischer und ein britischer Anteil aufrechterhalten wurden - aus sechs, seit Anfang Januar 1948 integrierten Bipartite Groups für Wirtschaft bzw. Handel und Industrie, Ernährung und Landwirtschaft, Verkehr, Post- und Fernmeldewesen, Finanzen und öffentlicher Dienst. Ebenfalls seit Januar 1948 waren die Bipartite Groups nicht mehr mit je einem Leiter britischer und amerikanischer Herkunft, sondern vielmehr nur noch mit einem Chef ausgestattet. Die Unterabteilungen (BICO Groups) für Commerce and Industry, Food, Agriculture and Forestry und Civil Service standen unter amerikanischer, die übrigen drei Unterabteilungen für Transport, Finance sowie Communication unter britischer Leitung. Den Stellvertreter stellte jeweils die andere Seite. Falls Meinungsverschiedenheiten zwischen einem Unterabteilungsleiter (Group Chief) und seinem Stellvertreter - formal also Streitpunkte zwischen den Vereinigten Staaten und Großbritannien - nicht überbrückt werden konnten, waren sie den beiden BICO Chairmen zur Entscheidung vorzulegen.

Für die Verwaltungstätigkeit von OMGUS ist die Feststellung zu treffen, da $B$ im Laufe der Zeit immer mehr Zuständigkeiten von den Fachabteilungen der amerikanischen Militärregierung in Berlin an das Bipartite Control Office bzw. ihm nachgeordnete deutsche Stellen übertragen wurden. Die gleichzeitige personelle Verringerung war auch darauf zurückzuführen, daß sich die Tätigkeit der amerikanischen Militärregierung hinsichtlich der Intensität der Überwachung deutscher Maßnahmen ebenfalls kontinuierlich verringerte.

Eine Darstellung der alliierten Zweizonenverwaltung wäre nicht vollständig, würde man die nachgeordneten Einrichtungen nicht erwähnen. Neben dem Military Security Board (Militärisches Sicherheitsamt) gab es die wirtschaftspolitisch hoch bedeutsame Joint Export Import Agency (JEIA), die Joint Foreign Exchange Agency (JFEA), die Allied Bank Commission, die Combined Coal Control Group, die Combined Steel Control Group und den Combined Travel Board.

An der Allied Bank Commission, der JEIA, der JFEA und dem Military Security Board war seit Herbst 1948 auch Frankreich beteiligt. Eine „eigentliche“ Dreimächte- 
Behörde entstand aber nur durch die Zusammenlegung von JEIA und Office du Commerce Extérieur (Oficomex) im Oktober 1948. Dann entwickelten sich regelmäßige Konferenzen der drei Militärgouverneure, die als Tripartite Board tagten.

Die innere Organisation von BICO veränderte sich im Frühjahr 1948 noch dadurch, daB eine Decartelization Commission, eine Legal Group und ein Bipartite Statistical Office, letzteres mit Sitz in Wiesbaden, geschaffen wurden. Auch bei dem Bipartite Statistical Office handelte es sich nicht um eine nachgeordnete Behörde, sondem um eine Verwaltungseinheit innerhalb des Bipartite Control Office. BICO wurde dann kurzfristig noch um eine Manpower Group, eine US Customs Group, eine Central Bank Group erweitert, doch hatten diese organisatorischen Änderungen auf die Verwaltung der amerikanischen Militärregierung, also von OMGUS, keinen entscheidenden Einfluß mehr.

Übersicht 18: Organisation und Personal von EUCOM/OMGUS, September 1947

\section{EUROPEAN COMMAND (EUCOM)}

Ofice of the Commander-in-Chief

Commander-in-Chief and Military Governor

Special Adviser

Political Adviser

Economics Adviser

Finance Adviser

Special Assistant

Budget and Fiscal Director

Director of Management Control

Director of Intelligence

Inspector General

Director of Civilian Personnel

Office of Military Government for Germany (US)

Commanding General, OMGUS, and Deputy

Military Governor, US-Zone

Assistant to Deputy Military Governor

Chief of Staff

Secretary General

Control Officer

Personnel Officer

Adjutant General

Audit Office, Chief

Director of Intelligence

Director of Political Affairs

Director of Public Information

$\begin{array}{ll}\text { Gen. } & \text { Lucius D.Clay } \\ \text { Civ. } & \text { J. Anthony Panuch } \\ \text { Amb. } & \text { Robert D. Murphy } \\ \text { Maj.Gen. } & \text { William H. Draper } \\ \text { Civ. } & \text { Jack Bennett } \\ \text { Civ. } & \text { F.L. Devereux } \\ \text { Col. } & \text { J.J.Dubbelde } \\ \text { Civ. } & \text { James L.Sundquist } \\ \text { Maj.Gen. } & \text { R.L.Walsh } \\ \text { Maj.Gen. } & \text { Louis A.Craig } \\ \text { Civ. } & \text { Robert M. Barnetı } \\ & \\ \text { Maj.Gen. } & \text { George P. Hays } \\ \text { Brig.Gen. } & \text { William Hesketh } \\ \text { Brig.Gen. } & \text { Charles K. Gailey } \\ \text { Col. } & \text { H.R. Maddux } \\ \text { Civ. } & \text { James L.Sundquist } \\ \text { Col. } & \text { James T.Duke } \\ \text { Lt.Col. } & \text { George H.Garde } \\ \text { Civ. } & \text { Moore C. Mcintosh } \\ \text { Col. } & \text { Peter P. Rodes } \\ \text { Civ. } & \text { Loyd V.Steere } \\ \text { Civ. } & \text { William R. Haggard }\end{array}$

\section{Armed Forces Division}

Director

Air Forces Branch, Chief

Civil Aviation Branch, Chief

Navy Branch (attached), Chief

Army Branch, Chief

$\begin{array}{ll}\text { Maj.Gen. } & \text { William E. Hall } \\ \text { Lt.Col. } & \text { D.E. Kunkel } \\ \text { Col. } & \text { G.M. Jones } \\ \text { Capt. } & \text { J.S.Crenshaw } \\ \text { Col. } & \text { T.B. Whitted }\end{array}$

\section{Civil Administration Division}

Director

Civ. Edward H. Litchfield

Deputy Director for Bizonal Affairs

Civ.

Kenneth A. Dayton

Civil Service and Administrative Courts Branch, Chief

Civ. 
Election and Political Parties Branch. Chief

Governmental Structures Branch. Chief

Legislation Branch. Chief

Policy Enforcement Branch, Chief

Population and Survey Branch. Chief

\section{Economics Division}

Director

Deputy Director for Administration

Deputy Director for Negotiations

Deputy Director for Bizonal Operations

Reports and Statistics Branch, Chief

Research Control Branch. Chief

Industry Branch. Chief

Trade and Commerce Branch, Chief

Decartelization Branch. Chief

Food and Agriculture Branch. Chief

Restitution Branch, Chief

\section{Finance Division}

Director

Deputy Director

Executive

Public Finance Branch, Chief

Property Control Branch, Chief

External Assets and Intelligence Branch. Chief

Foreign Exchange Depository (Frankfurt), Chief

Finance Institutions Branch, Chief

\section{Information Control Division}

Director

Deputy Director

Publications Control Branch, Chief

Radio Control Branch, Chief

Press Control Branch, Chief

Film, Theater, Music Control Branch, Chief

Research Branch, Chief

\section{Internal Afiairs and Communications Division}

Director

Deputy Director

Communications Branch, Chief

Public Safety Branch, Chief

Education and Religious Affairs Branch, Chief

Public Health Branch, Chief

Public Welfare Branch, Chief

\section{Legal Division}

Director

Associate Director

Board of Clemency, Chairman

Military Government Court for Civil Actions (Stuttgart), Judge

War Crimes Branch, Chief

Legislation Branch, Chief

Legal Advice Branch, Chief

$\begin{array}{ll}\text { Civ. } & \text { R.M. Scammon } \\ \text { Civ. } & \text { Hans Simons } \\ \text { Civ. } & \text { H. PhilipMettger } \\ \text { L..Col. } & \text { L.G. Kelly } \\ \text { Civ. } & \text { Karl A.Shafer }\end{array}$

Civ.

Lawrence Wilkinson

Col. Stephen A. Park

Civ.

Civ.

Civ.

Civ.

Civ.

Civ.

Civ.

Col.

Col.

Don D. Humphrey

A.S. Barrows

Saul Nelson

C.H.Nordstrom

H. L. Berno

Spencer Phenix

Philip Hawkins

Hugh B. Hester

John H. Allen

Civ.

Civ.

Civ.

Civ.

Civ.

Civ.

Col.

Civ.

Theodore H. Ball

Jo Fisher Freeman

Albert Bender

Howard P. Jones

E.N. Reinsel

Samuel M.Rose

William G. Brey

Richard P. Aikin

Col.

Civ.

Civ.

Civ.

Civ.

Civ.

Civ.

Gordon E. Textor

Thomas P. Headen

Douglas Waples

Charles S. Lewis

Arthur D. Eggleston

Eric T.Clarke

Robert C.Schmid

Civ.

Col.

George B.McKibbin

Emil Lenzner

Civ.

Civ.

Civ.

Col.

Civ.

Eugene H.Merrill

Theo E. Hall

Richard T. Alexander

Milford T.Kubin

Mildred L.C. Biklen

Civ.

Col.

Civ.

Civ.

Alvin J. Rockwell

John M. Raymond

J.J.Walsh

H. Gerhart

Civ.

Civ.

Ben Smith

H.E. Schopler

William E.McCurdy 
Administration of Justice Branch. Chief

Prison Branch. Chief

\section{Manpower Division}

Director

Labor Relations Branch, Chief

Wages and Labor Standards Branch. Chief

Social Insurance Branch, Chief

Manpower Allocation Branch. Chief

\section{Prisoners of War and Displeced Persons Division}

Director (Frankfurt)

Combined Repatriation Branch. Chief

Displaced Persons Branch, Chief

Refugees Branch, Chief

\section{Transport Division}

Director

Executive

Road Branch, Chief

Water Branch, Chief

Rail Branch, Chief

Movements Branch, Chief

Regional Government Coordinating Ofice (Stuttgart)

Director

\section{The Bipartite Organisations in the USVUK Zones}

Bipartite Boand (Located in Berlin)

US Member

Commander-in-Chief, EUCOM, and Military Governor.

US Zone

UK Member

Deputy Military Governor, British Zone

\section{Bipartite Secretariat (Located in Berlin)}

\section{US Chairman}

UK Chairman

Economics Group (Temporarily located in Minden)

US Member

Joint Export-Import Agency (Temporarily located in Minden)

\section{US Member}

UK Member

Finance Group (Located in Frankfurt)

US Member

UK Member

Food and Agriculture Group (Located in Frankfurt)

US Member

UK Member

Transport Group (Temporarily located in Bielefeld)

US Member

UK Member

$\begin{array}{ll}\text { Civ. } & \text { Haven Parker } \\ \text { Civ. } & \text { T. Wade Markley } \\ & \\ \text { Civ. } & \text { Leo R. Werts } \\ \text { Maj. } & \text { Harold F. Mullaney } \\ \text { Civ. } & \text { Sol D. Ozer } \\ \text { Civ. } & \text { M. G. Murray } \\ \text { Civ. } & \text { George S. Wheeler }\end{array}$

Brig.Gen. Thomas L. Harrold

Maj. John F. Asselta

Lt.Col. James P.Abbott

Col. Charles E. Boyle

$\begin{array}{ll}\text { Col. } & \text { Hans W. Holmer } \\ \text { Civ. } & \text { W. A. Fagan } \\ \text { Civ. } & \text { C.E. Calvert } \\ \text { Civ. } & \text { D. R. Neff } \\ \text { Civ. } & \text { E. V. Breitenbach } \\ \text { Civ. } & \text { J.C.Cook }\end{array}$

Lt.Col. Charles D. Winning
Lt.Gen. Sir Brian H. Robertson

Civ. Clarence L. Adcock

Lt.Gen. Sir Gordon N. Macready $\begin{array}{ll}\text { Civ. } & \text { A.S.Barrows } \\ \text { Brig.Gen. } & \text { J.G.Cowley }\end{array}$

Roy J. Bullock John F. Cahan

Civ.

Lt.Col. Emery D.Stoker

Civ.

J. T. Lisle

$\begin{array}{ll}\text { Civ. } & \text { J.C.Lynn } \\ \text { Civ. } & \text { H.P.Elder }\end{array}$

Col. A.H.Schroeder Maj.Gen. Sir Donald McMullen 
Communications Group (Located in Frankfurt)

\begin{tabular}{|c|c|c|}
\hline $\begin{array}{l}\text { US Member } \\
\text { UK Member }\end{array}$ & $\begin{array}{l}\text { Civ. } \\
\text { Civ. }\end{array}$ & $\begin{array}{l}\text { W.L.Slattery } \\
\text { Ronald W.C.Alford }\end{array}$ \\
\hline Civil Service Group (Located in Frankfurt) & & \\
\hline $\begin{array}{l}\text { US Member } \\
\text { UK Member }\end{array}$ & $\begin{array}{l}\text { Civ. } \\
\text { Maj. }\end{array}$ & $\begin{array}{l}\text { Ellsworth D. Wolfsperger } \\
\text { Vat A.C. Wade (retired) }\end{array}$ \\
\hline
\end{tabular}

Omice of the Chief of Counsel for War Crimes (Nümberg)

\section{Die Errichtung von EUCOM und die Verwaltungsreformen bei OMGUS im Jahre 1948}

Am 15. März 1947 hatte General Lucius D. Clay den bisherigen Oberbefehlshaber der amerikanischen Truppen in Europa (Commander-in-Chief of the United States Forces in the European Theater, USFET), General Joseph T. McNarney, abgelöst. Die Funktion des Deputy Military Governor und damit des Commanding General von OMGUS übernahm am selben Tag Generalmajor Frank A. Keating. Gleichzeitig wurde USFET in European Command (EUCOM) umbenannt und reorganisiert. Generalmajor Clarence R. Huebner wurde zum Deputy Commander-in-Chief, EUCOM, bestellt. $^{144}$

Unter EUCOM verstand man alle amerikanischen Einheiten und Kommandobehörden sowie sonstige Organisationen und Einrichtungen, die für die Sicherheit und sonstige logistische Unterstützung der amerikanischen Militärregierungen in Deutschland und Österreich verantwortlich waren. Der Stellvertreter des Commander-inChief, Generalmajor Clarence R. Huebner, war gleichzeitig Chef des Stabes bei EUCOM und Kommandierender General der amerikanischen Bodentruppen in Europa (Ground and Services Forces. Europe) mit Sitz in Frankfurt am Main. Stellvertretender Chef des Stabes bei EUCOM und Chef des Stabes der US Ground and Services Forces, Europe, war - ebenfalls in Frankfurt - Generalmajor Miller G. White. Er stützte sich auf einen Deputy Inspector General (Brigadegeneral William Hesketh), einen Deputy and Fiscal Director (Oberst Rutter), einen Director of Public Information (Oberst Eyster), und einen Secretary General Staff (Oberst Hill), die alle ebenso wie der Generalstab selbst in Frankfurt residierten. Der Generalstab gliederte sich wie üblich - in fünf Abteilungen.

\section{Übersicht 19: Organisation des General Staff, EUCOM, März 1947}

- G-1 Director of Personnel and Administration Maj.Gen. Bevans

- G-2 Deputy Director of Intelligence Col. Schow

- G-3 Director of Operations, Plans, Organization and Training Brig.Gen. Allen

- G-4 Director of Services, Supply and Procurement Maj.Gen. Magruder

- G-5 Director of Civil Affairs Maj.Gen. Thomas L. Harrold

${ }^{144}$ General Order Nr. 18 vom 15.3.1947. Vgl. Clay: Entscheidung in Deutschland, S. 258ff. Vgl. 2/135-1/36. 
Durch General Order Nr.66. EUCOM, vom 24.Juli 1947 wurde die Bezeichnung Headquarters, European Command. Berlin, in Office of the Commander-in-Chief, European Command, Berlin, umbenannt. ${ }^{145}$ Das Hauptquartier des European Command bestand im wesentlichen aus drei Teilen: dem Office of the Commander-in-Chief, European Command and Military Governor, in Berlin; dem Headquarters, European Command, in Frankfurt unter dem unmittelbaren Befehl des Deputy Commanderin-Chief and Chief of Staff. European Command, und schlieBlich dem Hauptquartier von OMGUS in Berlin unter dem Befehl des Deputy Military Governor and Commanding General, OMGUS. Aus Gründen der Arbeitsökonomie waren bestimmte Offiziere jedoch im Office of the Commander-in-Chief und im Headquarters, European Command, oder bei OMGUS oder sogar bei allen drei Stellen eingesetzt. Das Office of the Commander-in-Chief, European Command, gliederte sich seinerseits in mehrere, früher OMGUS zugeordnete Berater und den Director of Civilian Personnel, den Director of Management Control, den Director of Intelligence, den Inspector General und den Budget Fiscal Director. Die Dienststellenverwaltung von CINCEUR war OMGUS zugeordnet. In der Durchführung seiner Verpflichtungen als Militärregierung war OMGUS völlig unabhängig vom European Command. In Angelegenheiten militärischer Art war OMGUS jedoch nur eine von mehreren Kommandobehörden von EUCOM, welcher der Commander-in-Chief jedoch in Einzelfällen Privilegien im Verkehr mit den übrigen höheren Kommandobehörden gewährte.

Úbersicht 20: Organisation und Personal von EUCOM/OMGUS, Februar 1948 EUROPEAN COMMAND

Ofice of the Commander-in-Chief

Commander-in-Chief and Military Governor

Gen. Lucius D. Clay

Special Adviser

Civ. J. Anthony Panuch

Political Adviser

Amb. Robert D. Murphy

Finance Adviser

Civ. Jack Bennett

Special Assistant

Civ.

F. L. Devereux

Budget and Fiscal Director

Col.

Director of Management Control

Civ. James L.Sundquist

Management and Reports Branch. EUCOM, Chief

Civ.

Maj.Gen.

W. H. Schroder

Director of Intelligence

Inspector General

Maj.Gen.

R. L. Walsh

Director of Civilian Personnel

Civ.

Louis A.Craig

Robert M. Barnett

\section{OFFICE OF MILITARY GOVERNMENT FOR GERMANY (OMGUS)}

$\begin{array}{lll}\begin{array}{l}\text { Commanding General, OMGUS, and Deputy } \\ \text { Military Governor }\end{array} & \text { Maj.Gen. } & \text { George P.Hays } \\ \begin{array}{l}\text { Assistant to Deputy Military Governor } \\ \text { Chief of Staff }\end{array} & \begin{array}{l}\text { Brig.Gen. } \\ \text { Brig.Gen. } \\ \text { Secretary General }\end{array} & \begin{array}{l}\text { William Hesketh } \\ \text { Charles K.Gailey } \\ \text { H. R.Maddux }\end{array} \\ \begin{array}{l}\text { Control Office } \\ \text { Control Officer }\end{array} & \text { Civ. } & \text { James L.Sundquist } \\ \begin{array}{l}\text { Budget and Fiscal Control Branch, Chief } \\ \text { Graphics Branch, Chief }\end{array} & \text { Civ. } & \begin{array}{l}\text { Ralph D.Snow } \\ \text { Howard Denby }\end{array}\end{array}$

${ }^{145}$ AG 1947/122/5. Vgl. auch 5/458-2/31. 
Organization and Program Branch. Assistant Chief

Reports Branch, Chief

Statistics Branch. Chief

\section{Ofince of Personnel Ofincer}

Personnel Officer

Deputy Personnel Officer

Civilian Personnel Branch. Chief

Administrative Branch. Chief

Military Personnel Branch, Chief

Indigenous Personnel Branch. Chief

\section{Ofice of the Adjutant General}

Adjutant General

Miscellaneous Branch. Chief

Military Personnel Branch, Chief

Reference Library, Chief

Travel Branch, Chief

Combined Travel Branch, Chief

Publications Branch, Chief

Records Branch, Chief

Cable Control Branch, Chief

Message Control Branch, Chief

Ofifice of Inspector General

Inspector General

\section{Audit ofíice}

Chief

Ofince of Director of Intelligence

Director

Deputy Director

Analysis and Research Branch, Chief

Security Branch, Chief

Administration Branch, Chief

\section{Office of the Director of Political Afifirs}

Director

Political Branch, Chief

Economics Branch, Acting Chief

American Consulate General

\section{Public Information Ofifce}

Director

\section{Ofiice of Naval Adviser}

Naval Adviser

Deputy Naval Adviser

\section{Armed Forces Division}

Director

Deputy Director

Air Forces Branch, Chief

Army Branch, Chief

Civil Aviation Branch, Chief

Navy Branch (attached), Chief

Executive Branch, Chief

\begin{tabular}{|c|c|}
\hline $\begin{array}{l}\text { Civ } \\
\text { Civ. } \\
\text { Civ. }\end{array}$ & $\begin{array}{l}\text { Beryl R. McClaskey } \\
\text { Harold G.Carlson } \\
\text { Burnham P. Beckwith }\end{array}$ \\
\hline $\begin{array}{l}\text { Col. } \\
\text { Col. }\end{array}$ & $\begin{array}{l}\text { James T. Duke } \\
\text { John C. Arrowsmith }\end{array}$ \\
\hline $\begin{array}{l}\text { Civ. } \\
\text { Lt.Col. } \\
\text { Col. } \\
\text { Maj. }\end{array}$ & $\begin{array}{l}\text { John E. Tromer } \\
\text { Saul S. Dorfman } \\
\text { Louis G. Gibney } \\
\text { Henry W. Cornell }\end{array}$ \\
\hline Lt.Col. & George H.Garde \\
\hline $\begin{array}{l}\text { Capt. } \\
\text { Maj. } \\
\text { Civ. } \\
\text { Maj. } \\
\text { Civ. } \\
\text { I st Lt. } \\
\text { Capt. } \\
\text { Civ. } \\
\text { Capt. }\end{array}$ & $\begin{array}{l}\text { Clifford J.Storlie } \\
\text { Roscoe L. Bates } \\
\text { Henry A. Dunlap } \\
\text { John B. Mallon } \\
\text { Jean J. Chenard } \\
\text { Monroe M. Richardson } \\
\text { Robert E. Reed } \\
\text { Henry F. Roemmele } \\
\text { Homer C. Kelso }\end{array}$ \\
\hline Col. & Walter E. Jenkins \\
\hline Civ. & Moore C. McIntosh \\
\hline $\begin{array}{l}\text { Col. } \\
\text { Civ. }\end{array}$ & $\begin{array}{l}\text { Peter P.Rodes } \\
\text { Lawrence E. DeNeufville }\end{array}$ \\
\hline $\begin{array}{l}\text { Civ. } \\
\text { Civ. } \\
\text { WOJG }\end{array}$ & $\begin{array}{l}\text { Lawrence E. DeNeufville } \\
\text { Laughlin A.Campbell } \\
\text { Robert Baker }\end{array}$ \\
\hline
\end{tabular}

Civ. James W.Riddleberger

Civ. Warren M.Chase

Civ. Wesley C. Haraldson

Civ. Marshall M. Vance

Civ. William R. Haggard

Rear Adm. R. E.Schuirman

Capt. J. S. Crenshaw

Maj.Gen. William E. Holl

Col. T. B. Whitted

Lt.Col. D. E.Kunkel

Col. T. B. Whitted

Col. G. M.Jones

Capt. J. S. Crenshaw

Civ. G. B. Robbins 
Civil Administration Division

Director Civ

Adviser Civ

Deputy Director for Bizonal Affairs Civ.

Civil Service and Administrative Courts Branch. Chief Civ.

Election and Political Parties Branch. Chief Civ.

Governmental Structures Branch. Chief Civ.

Legislation Branch. Chief

Policy Enforcement Branch. Chief

Population and Survey Branch. Chief

Secretariat (Council of Foreign Ministers). Chief

Quadripartite Secretariat, Chief

\section{Economics Division}

Director

Deputy Director for Negotiations

Deputy Director for Administration

Deputy Director for Minden Operations

Administration and Personnel Branch, Chief

Economics Directorate Secretariat, Chief

Reports and Statistics Branch. Chief

Industry Branch, Chief

Trade and Commerce Branch, Chief

Food and Agriculture Branch. Chief

Decartelization Branch. Chief

Restitution Branch, Chief

Supply Accounting Branch, Acting Chief

\section{Finance Division}

Director

Deputy Director

Reports and Statistics Branch, Chief

Administrative Branch, Chief

Public Finance Branch, Chief

Property Control Branch, Chief

External Assets and Intelligence Branch, Chief

Foreign Exchange and Blocking Control Branch. Chief

Foreign Exchange Depository (Frankfurt), Chief

Finance Institutions Branch, Chief

\section{Information Control Division}

Director

Deputy Director

Plans and Directives Branch. Chief

Business Management Branch, Chief

Press Control Branch, Chief

Radio Control Branch, Chief

Film, Theater and Music Control Branch, Chief

Publications Control Branch, Chief

Exhibition and Information Centers Branch, Chief

Research Branch, Chief

\section{Internal Affairs and Communications Division}

Director

Deputy Director

Communications Branch, Chief iv.
Civ.
Civ
Civ.
Civ.

Civ.

Lt.Col.

Civ.

Civ.

Civ.

Civ.

Civ.

Col.

Civ.

Lt.Col.

Lt.Col.

Civ.

Civ.

Civ.

Col.

Civ.

Col.

Civ.

Civ.

Civ.

Civ.

Civ.

Civ.

Civ.

Civ.

Civ.

Col.

Civ.

Col.

Civ.

Civ.

Civ.

Civ.

Civ.

Civ.

Civ.

Civ.

Civ.

Civ.

Col.

Civ.
Edward H. Litchfield

John Elliot

Kenneth A.Dayton

Ellsworth D.Wolfsperger

R. M.Scammon

Hans Simons

H. Philip Mettger

L. G. Kelly

Karl A.Shafer

Alexander R. Forest

William Thad Lovelt

Lawrence Wilkinson

Don D. Humphrey

Stephen A. Park

A. S. Barrows

Thurman S. Doman

A. D. Poinier

Saul Nelson

H. L. Berno

Spencer Phenix

Hugh B. Hester

Philip Hawkins

John H. Allen

John Kovac

Theodore H. Ball

Jo Fisher Freeman

DavidSchwarty

J. B. A. Robertson

Howard P. Jones

E. N. Reinsel

Samuel M. Rose

Judson Jones

William G. Brey

Richard P. Aikin

Gordon E. Textor

Thomas P. Headen

Alfred V. Boemer

John Kidder

Arthur D. Eggleston

Charles S. Lewis

Eric T. Clarke

Douglas Waples

William C. Headrick

Robert C.Schmid

George B. McKibbin

Emil Lenzner

Eugene H.Merrill 
Education and Religious Affairs Branch. Chief

Public Health Branch. Chief

Public Safety Branch. Chief

Public Welfare Branch. Chief

\section{Legal Division}

Director

Associate Director

Consultant

Administration of Justice Branch. Chief

Board of Clemency. President

Legal Advice Branch, Chief

Legislation Branch, Chief

Prisons Branch, Chief

War Crimes Branch. Chief

Ofince of Chief of Counsel for War Crimes (Nürnberg)

Chief

\section{Manpower Division}

Director

Labor Relations Branch, Chief

Wages, Labor Standards and Housing Branch, Chief

Social Insurance Branch, Chief

Manpower Allocation Branch, Chief

\section{Prisoners of War and Displaced Persons Division}

Director

Secretariat, Administrative and Personnel Branch.

Chief

Refugees and PW Branch, Chief

DP and Stateless Persons Branch, Chief

Repatriation Branch (Combined Repatriation

Executive US Element ACA)

\section{Transport Division}

Director

Movements Branch, Chief

Rail Branch, Chief

Road Branch, Chief

Water Transport Branch, Chief

\section{Bipartite Agencies}

\section{Bipartite Boand}

US Member

UK Member

\section{Bipartite Secretariat}

US Secretary

US Deputy Secretary

\section{Bipartite Control Ofíce}

US Chairman

US Deputy Chairman

Economics Group (Minden)

US Member

$\begin{array}{ll}\text { Civ. } & \begin{array}{l}\text { Richard T. Alexander } \\ \text { Col. }\end{array} \\ \text { Civ. } & \text { Theo E. Hall } \\ \text { Civ. } & \text { Mildred B.Smith } \\ & \\ \text { Civ. } & \text { Alvin J. Rockwell } \\ \text { Col. } & \text { John M. Raymond } \\ \text { Civ. } & \text { Samuel Kramer } \\ \text { Civ. } & \text { Haven Parker } \\ \text { Civ. } & \text { J. J. Walsh } \\ \text { Civ. } & \text { William E. McCurdy } \\ \text { Civ. } & \text { H. E.Schopler } \\ \text { Civ. } & \text { T. Wade Markley } \\ \text { Civ. } & \text { Ben Smith }\end{array}$

Brig.Gen. Telford C. Taylor

$\begin{array}{ll}\text { Civ. } & \text { Leo R. Werts } \\ \text { Maj. } & \text { Harold F.Mullaney } \\ \text { Civ. } & \text { Sol D. Ozer } \\ \text { Civ. } & \text { M. G.Murray } \\ \text { Civ. } & \text { George S. Wheeler }\end{array}$

Brig.Gen. Thomas L. Harrold

Civ. Ezekiel L.Glazier

Col. Charles E. Boyle

Lt.Col. James P. Abbott

Maj. John F. Asselta

$\begin{array}{ll}\text { Col. } & \text { Hans W. Holmer } \\ \text { Civ. } & \text { J. C. Cook } \\ \text { Civ. } & \text { E. V. Breitenbach } \\ \text { Civ. } & \text { C. E. Calvert } \\ \text { Civ. } & \text { D. R. Neff }\end{array}$

Gen. Lucius D. Clay

Lt.Gen. Sir Brian H. Robertson

$\begin{array}{ll}\text { Maj. } & \text { Walter E. Mather } \\ \text { Maj. } & \text { Gordon L. C.Scott }\end{array}$

Civ. Clarence L. Adcock

Civ. Robert K. Phelps

Civ. A.S.Barrows 


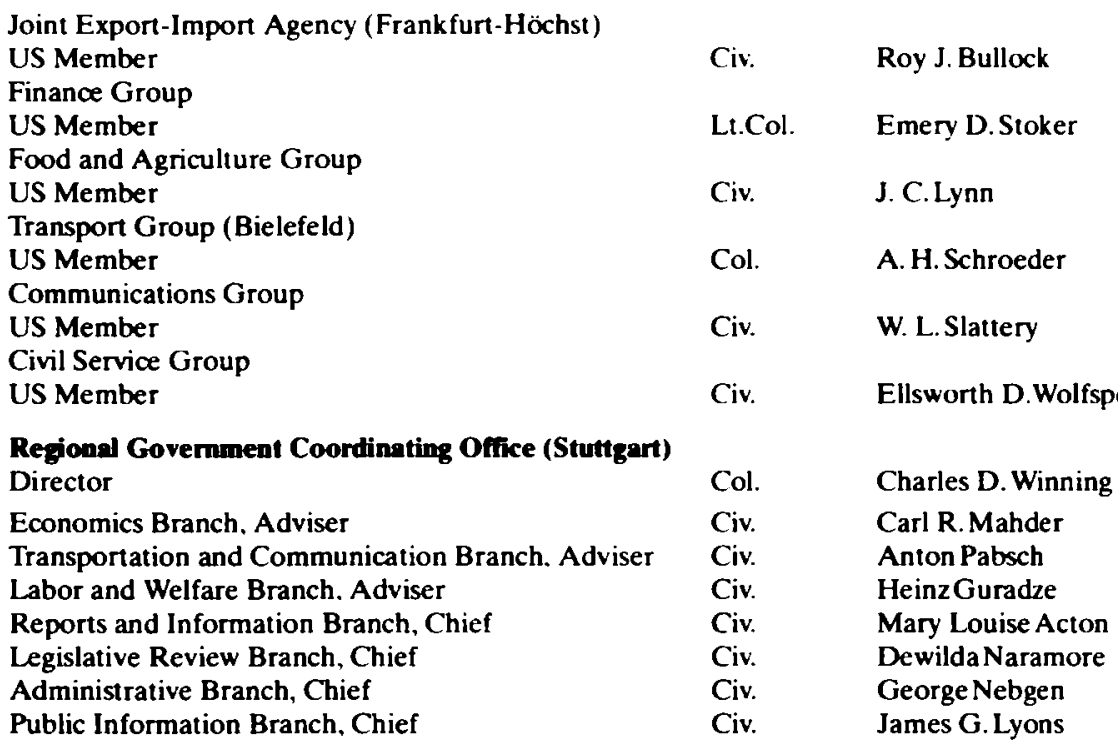

Durch General Order Nr. 18, EUCOM, wurden alle Fachabteilungen von OMGUS. die im weiteren Sinne für wirtschaftliche Angelegenheiten zuständig waren, mit Wirkung vom 1. März 1948 aufgelöst, ${ }^{146}$ da die Funktionen im wesentlichen entweder auf das Bipartite Control Office oder auf deutsche Zonen-bzw. Landesdienststellen übergegangen waren. Auf der anderen Seite übernahm OMGUS die Funktionen des Office of the Finance Adivser, CINCEUR, und des Office of the Economics Adviser. C.INCEUR, die beide aufgelöst wurden. Mit dieser Reform war eine erhebliche Personalverminderung verbunden. An die Stelle der Direktoren der Fachabteilungen traten nun Experten, die den Rang eines Beraters erhielten. Wie das Beispiel des bereits vorher bestehenden Naval Adviser zeigt, wurden diese Berater von einem Office bei ihrer Arbeit unterstützt. Daher gab es nunmehr bei OMGUS auch ein Office of the Finance Adviser und ein Office of the Economics Adviser. Diese beiden Ämter waren im Bereich der Aufgabenverwaltung im Unterschied zu anderen Offices nicht etwa in Branches, sondern in Groups gegliedert, die nicht oder nur in Ausnahmefällen mit den entsprechenden BICO Groups korrespondierten. So umfaßte das Office of the Finance Adviser unter Leitung des Finance Adviser to the Military Governor and Deputy Military Governor, Jack Bennett, sieht man von den Mitarbeitern in der unmittelbaren Umgebung des Beraters und im Bereich der fachübergreifenden Aufgaben einmal ab, folgende Gruppen:

- Foreign Exchange Policy Group (William S. Balderston)

- Internal Finance Policy Group (Walter Heller)

- Foreign Exchange Depository Group (Col. William G. Brey)

- Financial Intelligence Group (Saul Kagan).

Aus dem Bereich der alten Finance Division und der alten Economics Division wurden einige Kompetenzen in einer neuen Abteilung, der Property Division, unter Lei-

146 „Reorganization of OMGUS and CINCEUR Functions Relating to Finance. Economics, Transport, Communications, Restitution, Reparations, Decartelization and Property Control" ist überliefert in 5/357-1/14. Zur Property Division vgl. 5/357-1/29-30. 
tung von Philip Hawkins, dem bisherigen Leiter der Decartelization Branch in der Economics Division, zusammengefaßt. Außerdem wurde ein Special Consultant to the Deputy Military Governor with respect to affairs of the Property Division in der Person von Theodore H. Ball ernannt. Die neue Abteilung bestand aus folgenden Unterabteilungen:

- Reparations Branch (Orren R. McJunkins)

- Property Control and External Assets Branch (Fred E. Hartzsch) in Wiesbaden

- Restitution Branch (Col. John H. Allen) in Karlsruhe.

Hinzu kam die Central Filing Agency (Zentralmeldeamt) in Bad Nauheim.

Da die bisherige Wirtschaftsabteilung Zuständigkeiten an deutsche Stellen, an das Bipartite Control Office und an eine neue Fachabteilung der Militärregierung, die Property Division, verloren hatte, lag es nahe, dem Office of the Economics Adviser unter Leitung des Economics Adviser to the Military Governor and Deputy Military Governor, Lawrence Wilkinson, und des Deputy to the Economics Adviser, Don D. Humphrey, Zuständigkeiten über den Bereich der alten Wirtschaftsabteilung hinaus zuzuweisen.

Ubersicht 21: Organisation des Office of the Economics Adviser, OMGUS, Juni 1948

Economics Adviser

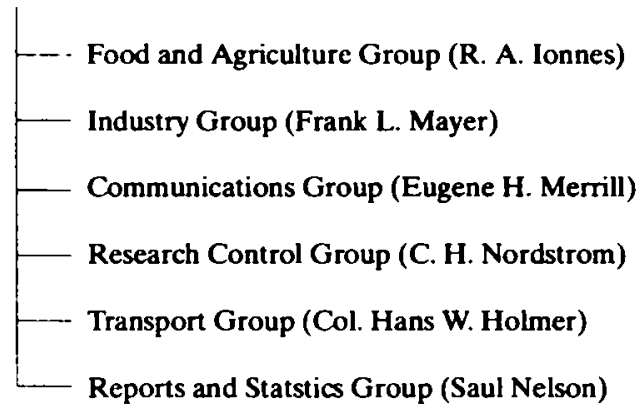

Aus dieser Aufstellung ergibt sich, daß die Transport Division der Militärregierung aufgelöst wurde. Die Erwähnung der Communications Group zeigt darüber hinaus an, da $\beta$ die organisatorischen Änderungen innerhalb der amerikanischen Militärregierung im Frühjahr 1948 auch andere Bereiche als den der Wirtschaft im weiteren Sinne betrafen. Durch General Order Nr.6 vom 18. Februar 1948 wurden die Civil Administration Division, die Internal Affairs and Communications Division und die Prisoners of War and Displaced Persons Division neu gegliedert oder aufgelöst. ${ }^{147}$ Der Aufgabenbereich der Civil Administration Division wurde neu geschnitten. Der überaus fähige Verwaltungsjurist Edward H. Litchfield ${ }^{148}$ blieb Abteilungsleiter, war auch weiterhin für die Unterabteilungen zuständig, welche die wesentliche Arbeit bei der Schaffung der politischen und administrativen Voraussetzungen für die Errichtung der Bundes-

147 „Reorganization of OMGUS Functions Affecting Internal Affairs and Communications, Civil Administration, and Prisoners of War and Displaced Persons Divisions“. Vgl. auch 5/357$1 / 11 ; 5 / 357-1 / 14 ; 5 / 363-1 / 15$.

148 Litchfield: Governing postwar Germany. 
Ubersicht 22: Organisation von EUCOM/OMGUS, März 1948

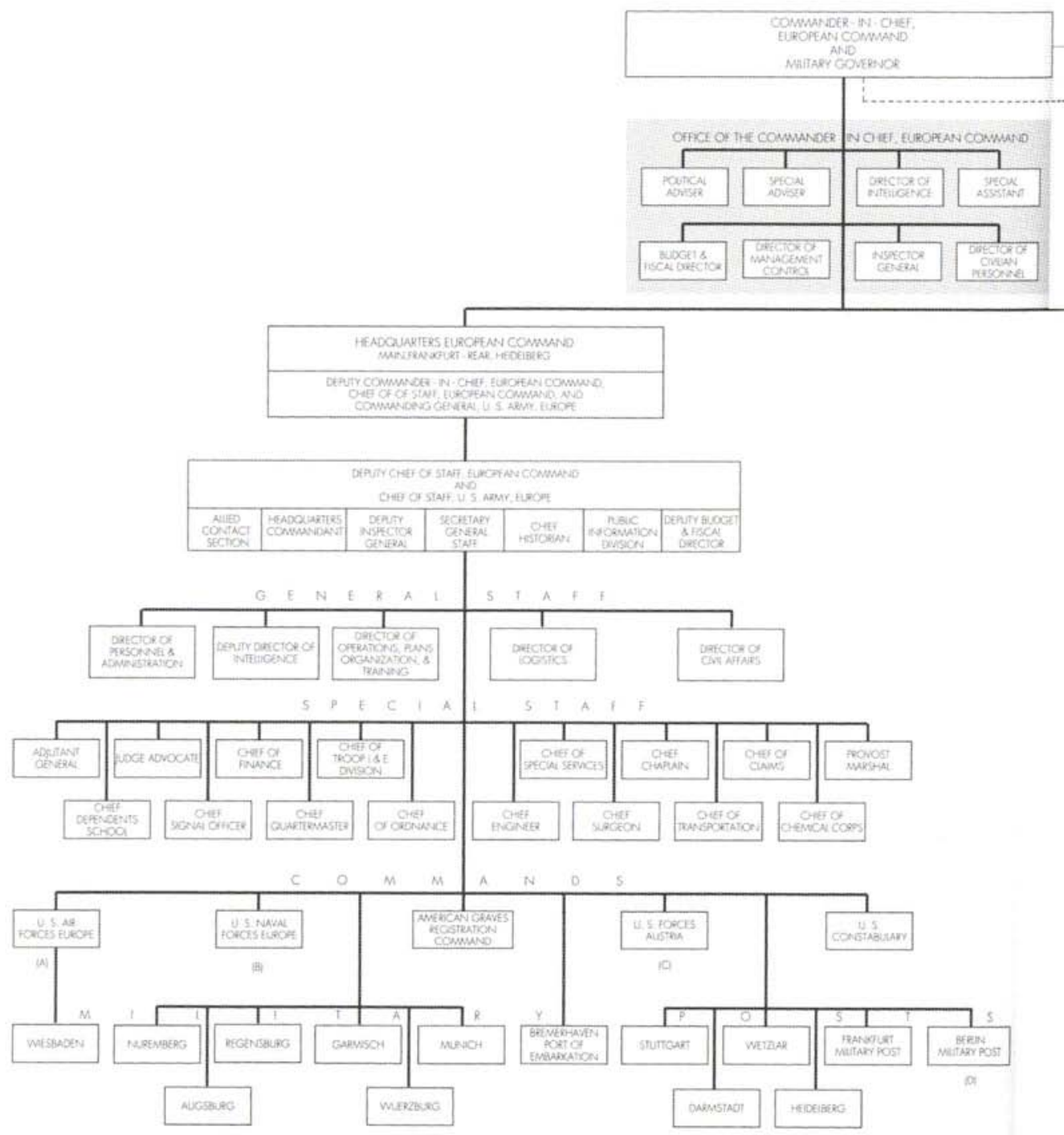

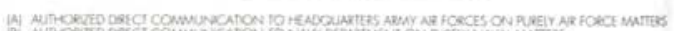
a efsonser petchiv to the 


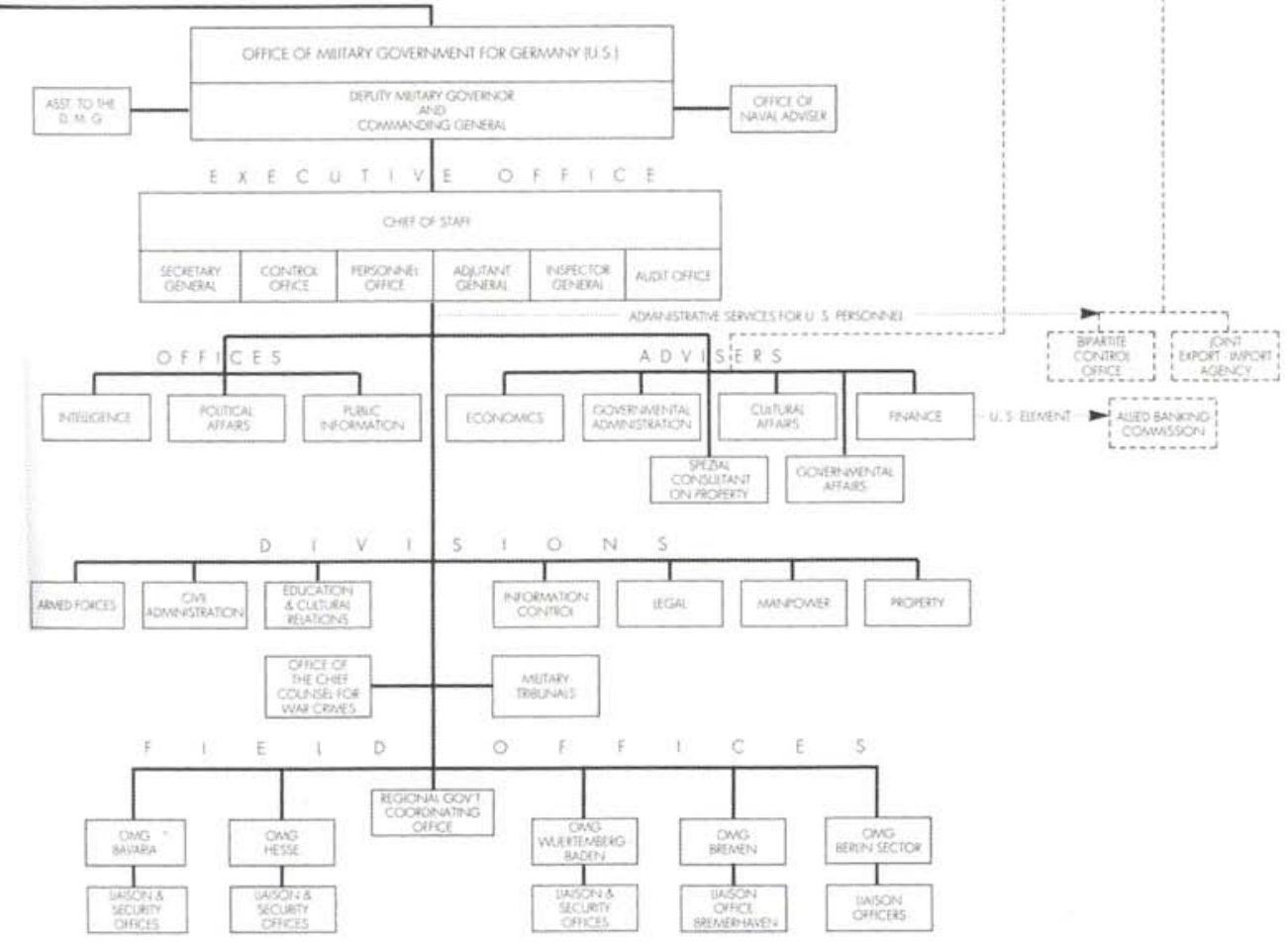


republik Deutschland leisten mußten, und erhielt zusätzlich die Unterabteilung für Gesundheitswesen (Public Health), Volkswohlfahrt (Public Welfare), öffentliche Sicherheit (Public Safety) sowie für Kriegsgefangene und Verschleppte (Prisoners of War and Displaced Persons). Schließlich übertrug man Litchfield auch die Zuständigkeiten im Bereich der inneren Verwaltung für Kontroll ratsangelegenheiten und damit die Vertretung von OMGUS im Internal Affairs and Communications Directorate des Viermächtekontrollorgans. Dabei hatte er sich je nach dem Gegenstand der Erörterung mit dem Leiter der neuen Education and Cultural Relations Division oder dem Economics Adviser abzustimmen. In diesem Zusammenhang zu erwähnen ist auch die Ernennung von Carl J. Friedrich zum Governmental Affairs Adviser ${ }^{149}$ Anfang April 1948. Hier zeigte sich erneut, mit welcher Intensität sich OMGUS auf die Errichtung eines westdeutschen Teilstaates vorbereitete.

In einem Memorandum vom 24. Juli 1948 bezeichnete der Control Officer, James L. Sundquist, die Verwaltungsreformen des Jahres 1948 als die erste Konsolidierung der OMGUS-Zentralverwaltung seit der Herausgabe des Organisationshandbuches vom 7. März 1946. ${ }^{150}$ Mitte 1948 bestand die OMGUS-Zentrale aus folgenden Einheiten:

Übersicht 23: Organisation von OMGUS, Mitte 1948

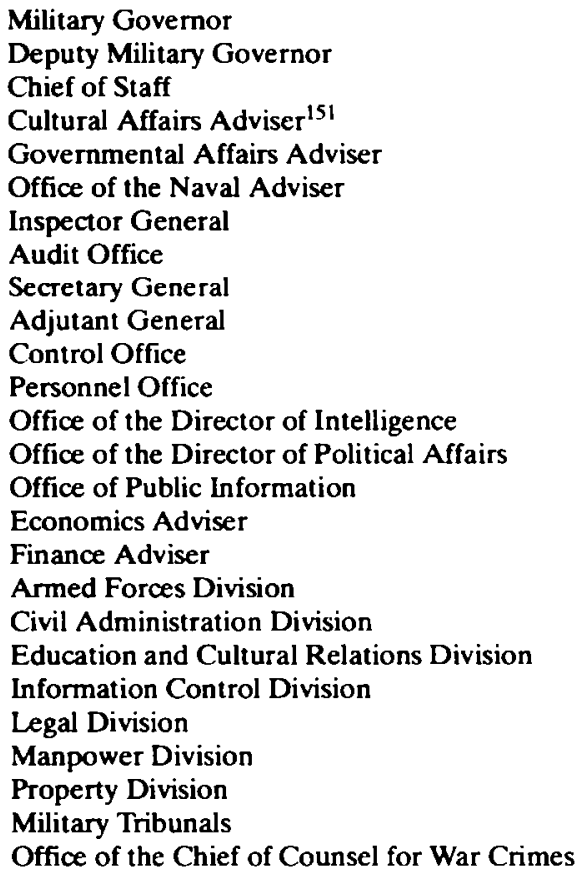

Ein offensichtliches Zeichen für die Setzung neuer Prioritäten der amerikanischen Militärregierung war die Schaffung der Education and Cultural Relations Division. Wäh-

149 General Order Nr. 13 vom 8.4.1948. Vgl. auch 5/357-1/11 und 5/357-1/14.

150 5/357-1/14.

151 Durch General Order Nr. 32 vom 9.8.1948 wurde die Position abgeschafft. Zur Education and Cultural Relations Division vgl. 5/364-2/28 und 5/365-1/22. 
rend Clay sich zu Beginn seiner Tätigkeit die Entsendung eines Cultural Adviser ausdrücklich verbeten hatte, war nun die wachsende Bedeutung nicht mehr zu übersehen, die insbesondere Schulfragen für die Politik von OMGUS inzwischen bekommen hatten. Hervorzuheben ist im kulturellen Bereich schließlich die Errichtung eines InterDivisional Reorientation Committee. ${ }^{152}$

Ubersicht 24: Organisation und Personal von CINCEUR/OMGUS, Juni 1948

\section{CINCEUR}

Commander-in-Chief and Military Governor

Political Adviser

Special Adviser

Special Assistant

Budget and Fiscal Director

Director of Management Control

Management and Reports Branch, EUCOM. Chief

Director of Intelligence

Inspector General

Director of Civilian Personnel

\section{OMGUS}

Commanding General, OMGUS, and Deputy Military Governor

Cultural Affairs Adviser

Governmental Affairs Adviser

Governmental Administration Adviser

Special Consultant of the Property Division

\section{Otice of Chief of Stafi}

Chief of Staff

\section{Ofice of Secretary General}

Secretary General

US Secretary, Bipartite Secretariat

Deputy US Secretary

\section{Control Office}

\section{Control Officer}

Assistant Control Officer for Bipartite Affairs

Budget and Fiscal Branch, Chief

Organization and Program Branch, Chief

Reports and Statistics Branch, Chief

\section{Ofince of Personnel Ofificer}

\section{Personnel Officer}

Administrative Branch, Chief

Civilian Personnel Branch, Chief

Military Personnel Branch, Chief

Indigenous Personnel Branch, Chief

\section{Office of the Adjutant General}

Adjutant General

Miscellaneous Branch, Chief

Military Personnel Branch, Chief

$\begin{array}{ll}\text { Gen. } & \text { Lucius D. Clay } \\ \text { Amb. } & \text { Robert D. Murphy } \\ \text { Civ. } & \text { J. AnthonyPanuch } \\ \text { Civ. } & \text { F. L. Devereux } \\ \text { Col. } & \text { J. J. Dubbelde } \\ \text { Civ. } & \text { James L.Sundquist } \\ \text { Civ. } & \text { W. H.Schroder } \\ \text { Maj.Gen. } & \text { R. L. Walsh } \\ \text { Maj.Gen. } & \text { Vernon Evans } \\ \text { Civ. } & \text { Robert M.Barnett } \\ & \\ \text { Maj.Gen. } & \text { George P. Hays } \\ & \\ \text { Civ. } & \text { Herman B. Wells } \\ \text { Civ. } & \text { Carl J.Friedrich } \\ \text { Civ. } & \text { George B. McKibbin } \\ \text { Civ. } & \text { Theodore H. Ball }\end{array}$

Brig.Gen. Charles K. Gailey

Civ. James E. King

Maj. Walter E.Mather

Maj. Gordon L. C. Scott

Civ. James L. Sundquist
Civ.

Civ. Fletcher D. Mitchel

Civ. Gordon P. Freese

Civ. H. R. Habicht

Col. James T. Duke

Civ. Rachael F. Jones

Civ. W. H.Carpenter

Maj. James M. Boyd

Civ. Gervaise M.Lemke

Lt.Col. George H.Garde

Capt. Clifford J.Storlie

Capt. Erwin M.Shaffer

152 General Order Nr. 29 vom 26.7.1948. 
Reference Library, Chief

Travel Branch. Chief

Combined Travel Board. Chief

Publications Branch, Chief

Records Branch, Chief

Cable Control Branch. Chief

Message Control Branch. Chief

Ofince of Inspector General

Inspector General

Audit Oniice

Chief

Deputy Chief

Ofice of Director of Intelligence

Director

Deputy Director

Analysis and Research Branch. Chief

Security Branch, Chief

Administration Branch. Chief

Ofíce of Director of Political Affairs

Director

Political Branch, Chief

Economics Branch, Chief

Civil Air Attaché

Press and Public Relations Branch, Chief

Consular Branch, Chief, Consul General

Public Information Ofíce

Director

Ofice of the Economics Adviser

Adviser

Deputy Economics Adviser

Deputy Adviser

Food and Agriculture Group, Chief

Industry Group, Acting Chief

Communications Group, Chief

Research Control Group, Chief

Tranport Group, Chief

Reports and Statistics Group, Chief

Ofirice of the Finance Adviser

Finance Adviser

Deputy Finance Adviser

Foreign Exchange Policy Group, Acting Chief

Internal Finance Policy Group, Chief

Central Bank Group, Deputy Chief

Foreign Exchange Depository Group, Chief

Financial Intelligence Group, Chief

Office of Naval Adviser

Commander of US Naval Forces in Germany and

Naval Adviser

Deputy Naval Adviser and Senior Technical Officer

\begin{tabular}{|c|c|}
\hline $\begin{array}{l}\text { Civ. } \\
\text { Maj. } \\
\text { Civ. } \\
\text { Civ. } \\
\text { Capt. } \\
\text { Civ. } \\
\text { Capt. }\end{array}$ & $\begin{array}{l}\text { Henry A. Dunlap } \\
\text { John B.Mallon } \\
\text { Jean J.Chenard } \\
\text { Monroe M. Richardson } \\
\text { Robert E. Reed } \\
\text { Charles R. Bakey } \\
\text { Homer C. Kelso }\end{array}$ \\
\hline Col. & Walter E. Jenkins \\
\hline Civ. & $\begin{array}{l}\text { (vacant) } \\
\text { Walter P. Hooper }\end{array}$ \\
\hline $\begin{array}{l}\text { Col. } \\
\text { Civ. }\end{array}$ & $\begin{array}{l}\text { Peter P. Rodes } \\
\text { Lawrence E. DeNeufville }\end{array}$ \\
\hline $\begin{array}{l}\text { Civ. } \\
\text { Civ. } \\
\text { WOJG }\end{array}$ & $\begin{array}{l}\text { Lawrence E. DeNeufville } \\
\text { Laughlin A.Campbell } \\
\text { Robert Baker }\end{array}$ \\
\hline
\end{tabular}

Civ.

James W. Riddleberger

Civ.

Civ.

Civ.

Civ.

Civ.

Civ.

Warren M. Chase

James W. Gantenbein

Milton M. Turner

Thomas J. B. Wenner

Marshall M. Vance

William R. Haggard

Civ.

Civ.

Civ.

Civ.

Civ.

Civ.

Civ.

Col.

Civ.

Lawrence Wilkinson

Philip Hawkins

Don D. Humphrey

R. A. Ionnes

Frank L. Mayer

Eugene H.Merrill

C. H.Nordstrom

Hans W. Holmer

Saul Nelson

$\begin{array}{ll}\text { Civ. } & \text { Jack Bennett } \\ \text { Civ. } & \text { Jo Fisher Freeman } \\ \text { Civ. } & \text { William S. Balderston } \\ \text { Civ. } & \text { Walter Heller } \\ \text { Civ. } & \text { Henry C. Conrad } \\ \text { Col. } & \text { William G. Brey } \\ \text { Civ. } & \text { Saul Kagan }\end{array}$

Rear Adm. R. E.Schuirman

Capt. J.S. Crenshaw 


\section{Armed Forces Division}

Director

Deputy Director

Air Forces Branch. Chief

Army Branch, Chief

Civil Aviation Branch, Chief

Meteorological Branch. Chief

Navy Branch (attached). Chicf

Executive Branch, Chief

\section{Civil Administration Division}

Director

Adviser

Deputy Director for Bizonal Affairs

Deputy Director for Operations

CFM Secretariat Branch, Chief

Personnel and Administration Branch, Chief

Civil Service and Administrative Courts Branch, Chief

Political Activities Branch, Chief

Governmental Structures Branch, Chief

Legislation and Analysis Branch, Chief

Population and Analysis Branch, Chief

Public Safety Branch, Chief

Public Welfare Branch

Displaced Persons Branch, Chief

Civil Liberties and Democratization Branch, Chief

\section{Education and Cultural Relations Division}

Director

Deputy Director

Cultural Exchange Branch. Acting Chief

Education Branch, Chief

Group Activities Branch, Chief

Religious Affairs Branch, Chief

\section{Information Control Division}

Director

Deputy Director

Deputy Director for Policy

Exhibition and Information Centers, Chief

Film, Theater and Music Branch, Chief

Opinion Surveys Branch, Chief

Overt Management Branch

Deputy for Quadripartite Affairs, Plans and Directives Branch, Chief

Deputy for Zonal Affairs

Political Information Branch, Chief

Press Branch, Nümberg, Chief

Publications Branch, Nümberg, Chief

Radio Branch, Chief

\section{Legal Division}

Director

Associate Director

Deputy Director

$\begin{array}{ll}\text { Maj.Gen. } & \text { William E. Hall } \\ \text { Col. } & \text { T.B. Whitted } \\ \text { Lt.Col. } & \text { B.E.Steadman } \\ \text { Col. } & \text { T.B. Whitted } \\ \text { Civ. } & \text { Thomas D.Johnson } \\ \text { Civ. } & \text { Clayton Van Thullenar } \\ \text { Capt. } & \text { J.S.Crenshaw (US Navy) } \\ \text { Civ. } & \text { G.B.Robbins }\end{array}$

Civ.

Civ.

Civ.

Civ.

Civ.

Civ.

Civ.

Civ.

Civ.

Civ.

Civ.

Civ.

Civ.

Civ.

Civ.

Edward H. Litchfield

John Elliot

Kenneth A. Dayton

H. Philip Mettger

Alexander R. Forest

F. Joseph Tesmer

Ellsworth D.Wolfsperger

R. M.Scammon

Hans Simons

Erwin W.Bard

Karl A.Shafer

Theo E. Hall

Mildred B.Smith

L. G. Kelly

Harold W. Landin

Civ.

Civ.

Civ.

Civ.

Civ.

Civ.

Alonzo G.Grace

Milton E. Muelder

Frank G.Banta

Richard T. Alexander

Lawrence E. Norrie

C. Arild Olsen

Col.

Civ.

Civ.

Civ.

Civ.

Civ.

Civ.

Civ.

Civ.

Civ.

Civ.

Civ.

Gordon E. Textor

Thomas P.Headen

Alfred V.Boemer

Patricia Van Delden

Eric T.Clarke

Frederick W. Williams (vacant)

Herbert C.Mayer

C. C.Baldwin

Lawrence F. Babcock

Peter Olden

Douglas Waples

Charles S. Lewis

Col.

John M. Raymond

(vacant)

Civ. 
Administration of Justice Branch. Nürnberg. Chief Legal Advice Branch. Chief

Legislation Branch. Chief

Prisons Branch, Nürnberg, Chief

War Crimes Branch, Nürnberg, Chief

\section{Menpower Division}

Director

Wages \& Labor Standards Branch, Chief

Labor Relations Branch, Chief

Reports and Statistics Branch, Chief

\section{Property Division}

Director

Deputy Director

Reparations Branch, Chief

Property Control and External Assets Branch. Wiesbaden, clo OMG Hesse

Central Filing Agency, Bad Nauheim, Chief

Restitution Branch, Karlsruhe, Chief

\section{Oilice of Chief of Coansel for War Crimes (Nürmberg) \\ Chief of Counsel}

Deputy Chief of Counsel (I.G.Farben)

Deputy Chief of Counsel (Krupp Case)

Deputy Chief of Counsel (Ministries Case)

Deputy Chief of Counsel (High Command Case)

Administrative Division, Director

Reproductions Division, Director

Signal Division, Director

Language Division, Director

SS and Military Division, Director

Evidence Division, Director

Economic Ministries Division, Director

Political Ministries Division, Director

Special Projects Division, Director

Public Relations Division, and Public Information Officer

\author{
Military Tribunals \\ Secretary General of Tribunals \\ Military Tribunal III (Krupp Case) \\ Presiding Judge \\ Military Tribunal IV (Ministries Case) \\ Presiding Judge \\ Military Tribunal V (High Command Case) \\ Presiding Judge \\ Military Tribunal VI (I.G. Farben Case) \\ Presiding Judge
}

\section{Bipartite Board}

US Member

\section{Bipartite Secretariat \\ US Secretary}

\section{Bipartite Control Office}

US Chairman

\begin{tabular}{|c|c|}
\hline Civ. & Charles H. Kraus \\
\hline Civ. & James E. Heath \\
\hline Civ. & Creighton R.Coleman \\
\hline Civ. & T. Wade Markley \\
\hline Civ. & Ben Smith \\
\hline Civ. & Leo R. Werts \\
\hline Civ. & Earl R. Beckner \\
\hline Civ. & John K.Meskimen \\
\hline Civ. & Albert H. Berman \\
\hline Civ. & Philip Hawkins \\
\hline Civ. & E. J.Cassoday \\
\hline Civ. & Orren R.McJunkins \\
\hline Civ. & Fred E. Hartzsch \\
\hline Civ. & Bernard Fischbein \\
\hline
\end{tabular}

Brig.Gen. Telford C. Taylor

Civ.

Civ.

Civ.

Civ.

Capt.

Maj.

Maj.

Civ.

Civ.

Civ.

Civ.

Civ.

Civ.

Civ.

Civ.

Josiah E. Dubois

Rawlings Ragland

Robert M. W. Kempner

James M. McHaney

Donald Paul

Alexander C. Granzin

Kenneth K. Evans

Thomas K. Hodges

James M. McHaney

Walter H. Rapp

H. Russell Thayer

Robert M.W.Kempner

Paul H. Gantt

Emest D. Deane

Howard L. Russell

Hu. C. Anderson

William C. Christiansen

John C. Young

Curtis G.Shake

Gen.

Lucius D. Clay

Maj. Walter E. Mather

Civ. Clarence L. Adcock 
UK Chairman

US Deputy Chairman

UK Deputy Chairman

Ofice of US Chrirman

Governmental Affairs Adviser

US Secretariat, Chief

\section{Decartelization Commiscion}

US Member

UK Member

\section{Civil Service Group}

Chief (US)

Deputy Chief (UK)

\section{Commerce and Industry Group}

Chief (US)

Deputy Chief (UK)

Communications Group

Chief (UK)

Deputy Chief (US)

\section{Finance Group}

Chief (UK)

Deputy Chief (US)

Food, Agriculture and Forestry Group

Chief (US)

Deputy Chief (UK)

\section{Transport Group}

Chief (UK)

Deputy Chief (US)

\section{Legal Group}

Chief (US)

Deputy Chief (UK)

Bipartite Statistical Onice (Wiesbaden)

US Chairman

UK Chairman

Joint Export-Import Agency (Höchst)

Director General (US)

Deputy Director General (UK)

Joint Foreign Exchange Agency (Frankfurt)

US Member

\section{Alied Bank Commission \\ US Member \\ Alternate}

\section{US-UK Coal Control Group (Essen)}

US Chairman

Regional Government Coordinating Ofice (Stuttgart)

Director

Senior Adviser
Lt.Gen. Sir Gordon N. Macready

Civ. Robert K. Phelps

Civ. D. L.Anderson

Civ. Kenneth A. Dayton

Civ. Emest Linde

Civ. Richard Bronson

Brig. Oxborrow

Civ. R. C.Simonson

Maj. Vat A.C.Wade

Civ. L. E.Spencer

Civ. W. L. Tregoning

Civ. L. G.Semple

Civ. C. P. Rapp

Civ. Joseph Charles

Brommage

Civ. Howard P. Jones

Civ. Stanley Andrews

Civ. Guy Erskine Hughes

Civ. Sir Robert Inglis

Civ. C. P.Gross

Civ. Richard D. Keamey

Civ. Harcourth Rose

Civ. H. H. Lee

Civ. F. R. Yates

Civ. William John Logan

Civ. John F. Cahan

Civ. Jo Fisher Freeman

Civ. Jack Bennett

Civ. Jo FisherFreeman

Civ. Robert R. Estill

Civ. Charles D. Winning

Civ. Anton Pabsch 
Economics Branch, Adviser

Labor and Welfare Branch. Adviser

Legislative Review Branch, Adviser

Reports and Information Branch, Chief

Public Information Branch. Chief

$\begin{array}{ll}\text { Civ. } & \text { Carl R.Mahder } \\ \text { Civ. } & \text { HeinzGuradze } \\ \text { Civ. } & \text { Dewilda Naramore } \\ \text { Civ. } & \text { Myrtle Todd Moseley } \\ \text { Civ. } & \text { James G.Lyons }\end{array}$

Im Juli 1948 unterschied sich der Aufbau der Fachabteilungen von OMGUS wesentlich von der Organisationsstruktur, die noch im Februar desselben Jahres bestanden hatte. Die Zahl der Abteilungen war formal von zehn auf sieben, de facto jedoch nur auf neun gesunken. Den aufgelösten Abteilungen (Economics. Finance, Internal Affairs and Communications. Transport, Prisoners of War and Displaced Persons) standen zwei neue Abteilungen (Property, Education and Cultural Relations) gegenüber. Berücksichtigt man jedoch, daß die Abteilungen für Finanzen und Wirtschaft durch Fachberater mit eigenen Offices auf diesen beiden Gebieten ersetzt wurden, so wird deutlich, daß die Zahl der Verwaltungseinheiten nur von zehn auf neun zurückgegangen, eine Beschleunigung des Geschäftsganges und eine Straffung der Verwaltungstätigkeit also nur bedingt zu erwarten waren. Dabei kann offen bleiben, ob dieses Ziel überhaupt verfolgt wurde. Aus diesen Überlegungen ergibt sich, daß neue politische Prioritäten zu organisatorischen Konsequenzen geführt hatten, wie sich vor allem am Beispiel der neuen Civil Administration Division darstellen ließ.

Politische Überlegungen dürften auch bei den organisatorischen Veränderungen in der Information Control Division eine Rolle gespielt haben. Statt der Press Control Branch gab es nun eine Press Branch, statt der Radio Control Branch eine Radio Branch, statt der Publication Control Branch eine Publication Branch und statt der Film, Theater and Music Control Branch eine Film, Theater and Music Branch. Durch General Order Nr.31 vom 6. August 1948 wurde auch im Namen der Abteilung selbst das Wort ..Control“ gestrichen. ${ }^{153}$ Im Zeichen einer deutlich liberaler gewordenen Besatzungspolitik war diese Vokabel offensichtlich nicht mehr angemessen. Das Nachlassen der unmittelbaren Kontrolle der Besatzungsmacht gegenüber den deutschen Dienststellen, insbesondere auch im Verhältnis zum Länderrat in Stuttgart, und der Ausbau der Bizone führten dazu, daß das amerikanische Pendant zum Länderrat, das Regional Government Coordinating Office, durch General Order Nr.24 vom 1.Juli 1948 aufgelöst und seine restlichen Aufgaben von der OMGUS-Zentrale unmittelbar wahrgenommen wurden.

Im August 1948 gehörten zum Office of the Commander-in-Chief, EUCOM, ${ }^{154}$ ein Political Adviser (Robert D. Murphy), ein Special Adviser (J. Anthony Panuch), ein Director of Intelligence (Maj.Gen. R. L. Walsh) ein Inspector General (Maj.Gen. Vernon Evans), ein Special Assistant (F. L. Devereux), ein Budget and Fiscal Director (Col. J. J. Dubbelde), ein Director of Civilian Personnel (Robert M. Barnett) und ein Director of Management Control (James L. Sundquist). Naval Adviser Clays war Rear Admiral R. E. Schuirman, Deputy Commander-in-Chief, Chief of Staff, EUCOM, and Commanding General, US Army, Europe, Maj.Gen. Clarence R. Huebner.

Als Chief of Staff, OMGUS, war - unabhängig vom Chief of Staff, EUCOM, Brig.Gen. Charles K. Gailey eingesetzt, unter dem als Secretary General James E. King fungierte. Eine bemerkenswerte Personalunion, die nicht zufällig zustande gekommen sein kann, bestand dadurch, daß der Director of Management Control, EU-

153 General Order Nr. 31 vom 6.8.1948.

154 5/357-1/11 und 5/357-1/14. 
COM. Sundquist. gleichzeitig Control Officer, OMGUS, war; dies läßt auf einen sehr starken Rückhalt von Sundquist bei Clay schließen. Personnel Officer, OMGUS, war damals Col. James T. Duke. Adjutant General Lt.Col. George H. Garde. Inspector General Col. W. E. Jenkins und Audit Officer H. L. Karns: sie alle verfügten nicht über Funktionen bei EUCOM.

Auch im Bereich der ..Außenpolitik“ gab es neben dem Political Adviser. EUCOM. Murphy, einen Director of Political Affairs. OMGUS, James W. Riddleberger, in dessen Amt (Office) fünf Unterabteilungen (Branches) bestanden: Political (im engeren Sinne), Economics, Civil Air (Attache), Press and Public Relations und Consular. Auch das Office of the Director of Intelligence, OMGUS, unter Peter P. Rodes und das Public Information Office, OMGUS, unter William R. Haggard bestanden ohne personale Bindung der Spitzenposition zu EUCOM.

Durch Verordnung Nr. 33 vom 18. A ugust 1948 wurde das Militärgerichtswesen reformiert. ${ }^{155}$ indem die Gerichtsorganisation im amerikanischen Kontrollgebiet Deutschlands in elf Gerichtsbezirke gegliedert wurde.

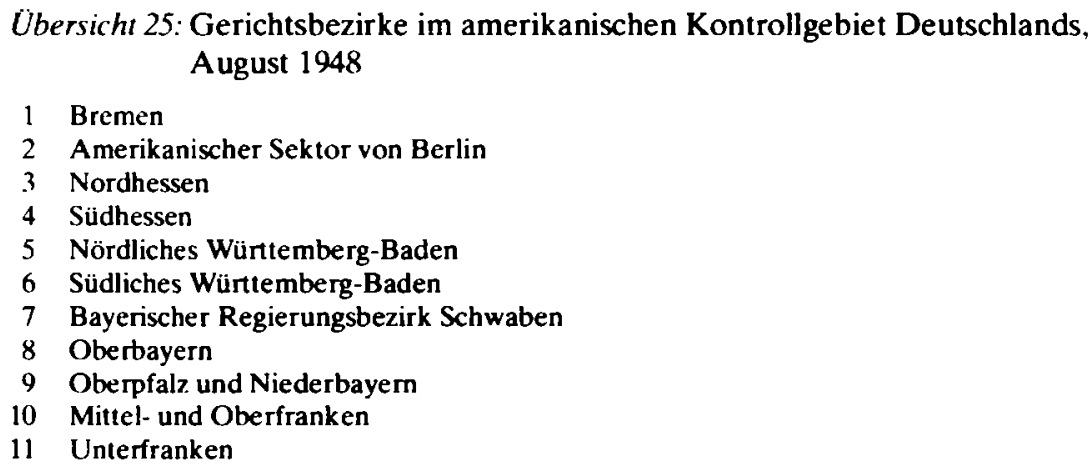

Für jeden Bezirk wurde ein Bezirksgericht (District Court) errichtet. Berufungsgericht war der Court of Appeals. Die Gerichte waren u. a. auch als Rheinschiffahrtsgerichte zuständig. Hinzu kamen entsprechende Staatsanwälte (Attorneys). Die Dienstaufsicht übte der Deputy Military Governor, Maj.Gen. George P. Hays, durch den Chief of Staff, OMGUS, aus. Die oberste Anklagevertretung übernahm das Office of the Chief Attorney, das im Rahmen der Legal Division errichtet wurde.

Im Bereich der Fachämter (Functional Offices) ergab sich durch General Order Nr. 37 vom 18. September 1948 insofern eine verwaltungsgeschichtlich bedeutsame Änderung, als das Audit Office aufgelöst und seine Aufgaben der Budget and Fiscal Branch des Control Office übertragen wurden. ${ }^{156}$ Noch wichtiger war indessen die Errichtung eines Public Informations Office durch General Order Nr. 48 vom 2. November 1948 sowie eines Newspaper Leases Review Board durch General Order Nr. 15 vom 23. Mai 1949.

Für die allgemeine politische Entwicklung in der amerikanischen Besatzungszone zumindest während der zweiten Hälfte des Jahres 1948 - ist es bezeichnend, daß mehrere Verwaltungseinheiten von OMGUS aus Berlin in die amerikanische Zone verlegt wurden. Diese Entwicklung ist vor dem Hintergrund der Fortschritte zu sehen, die der

155 Military Government Gazette. Ausgabe K, S. 35 ff.

156 5/357-1/15. 


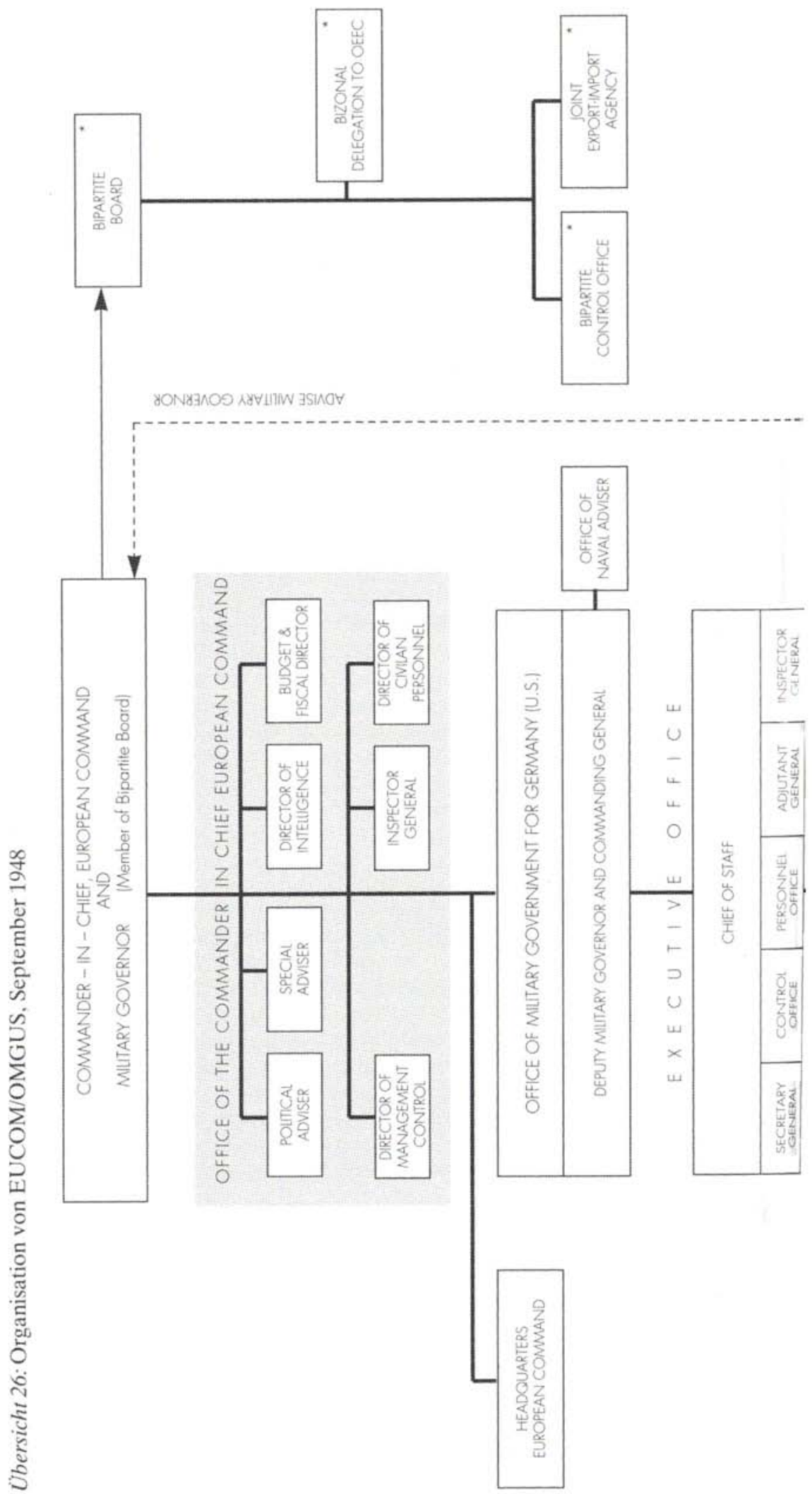




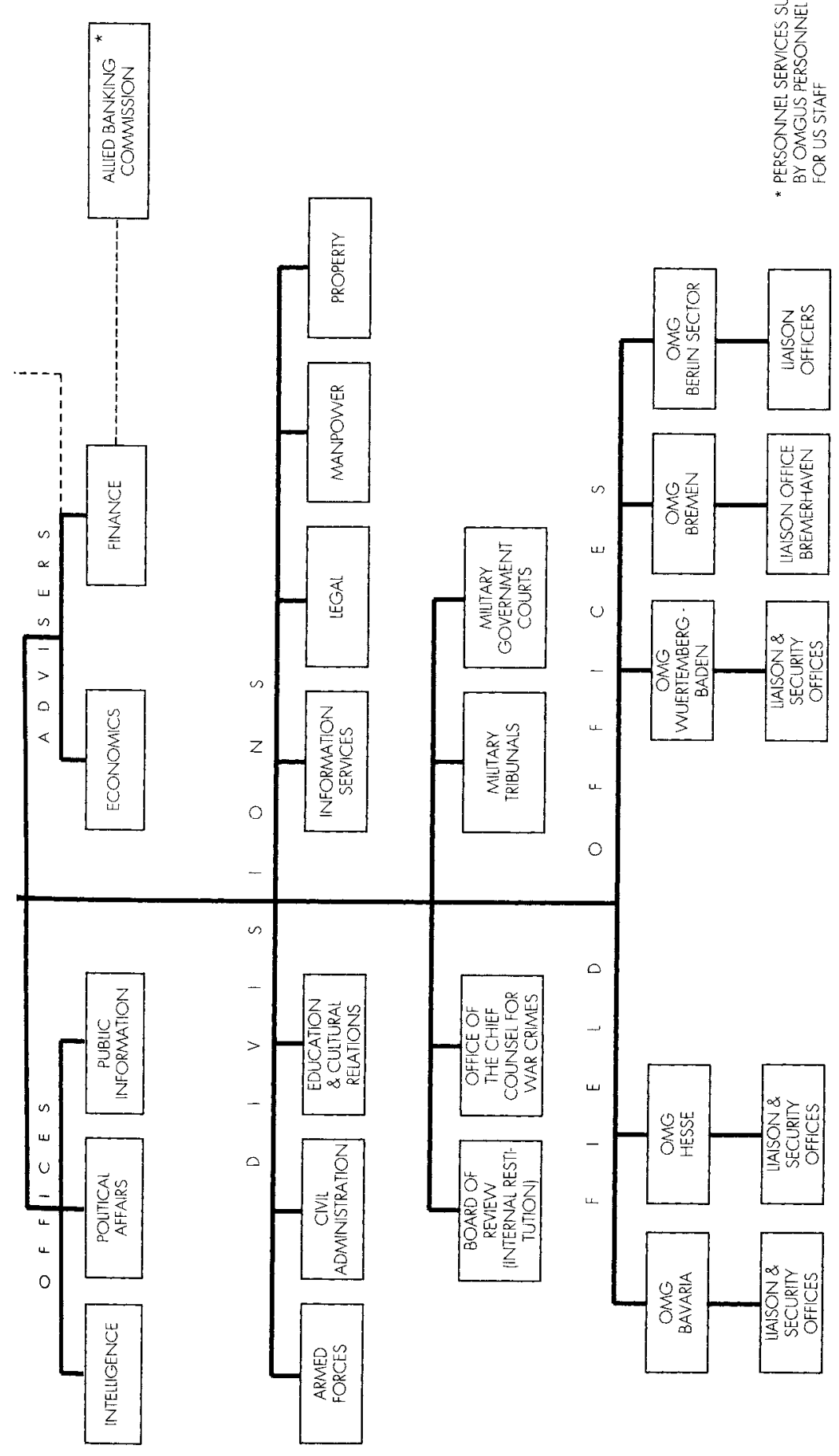


Aufbau der (deutschen) bizonalen Verwaltung gemacht hatte. Die räumliche Nähe zur entsprechenden deutschen Behörde wog für die amerikanische Seite die organisatorischen Nachteile einer Trennung von Berlin und damit von der OMGUS-Zentrale offensichtlich mehr als auf. Dabei wurden die in die amerikanische Zone verlegten Verwaltungseinheiten administrativ oft regionalen Dienststellen angegliedert, so z. B. die Motion Pictures Branch der Dienststellenverwaltung des Office of Military Government for Bavaria. ${ }^{157}$ Bezeichnend für diese Entwicklung ist die Errichtung eines Office of Deputy Chief of Staff, OMGUS, in Nümberg am 1. November 1948. ${ }^{158} \mathrm{Mit}$ Wirkung vom 21.März 1949 wurde diese Dienststelle ebenso wie die Education and Cultural Relations Division nach Bad Nauheim verlegt.

Sieht man von den bereits erwähnten Maßnahmen ab, so ergaben sich zwischen August 1948 und Januar 1949 folgende Änderungen im Aufbau der Zentralverwaltung der Militärregierung: ${ }^{159}$

1. Errichtung einer Historical Branch im Control Office, die sich nicht zuletzt der Vorbereitung von Clays Memoiren zu widmen hatte.

2. Aufwertung der Political Branch im Office of the Director of Political Affairs durch Übernahme der Zuständigkeit des Verkehrs mit ausländischen Vertretungen.

3. Errichtung einer Meteorological Branch in der Armed Forces Division.

4. Übergang der Kompetenz für Kunst, Museen, Bibliotheken und Archive auf die Education and Cultural Relations Division.

5. Auflösung der War Crimes Branch in der Legal Division und Errichtung eines Military Government Court for Civil Actions.

6. Neustrukturierung der Manpower Division.

7. Errichtung eines Board of Review for Internal Restitution.

Im Bereich der Fachabteilungen (Functional Divisions) von OMGUS gab es im Laufe des Jahres 1949 noch zwei wesentliche Änderungen, die Zweifel daran rechtfertigen, ob eine tragfähige Theorie eines funktionsgerechten Verwaltungsaufbaus den jeweiligen Entscheidungen zugrunde lag. Durch General Order vom 7. April 1949 wurde die Finance Division unter Leitung von Jo Fisher Freeman wieder errichtet. Eher verständlich ist dagegen - mindestens bei isolierter Betrachtung - die Entscheidung, die Property Division mit Wirkung vom 1.Juli 1949 aufzulösen und durch eine Property Group im Office of the Economics Adviser zu ersetzen. ${ }^{160}$

\section{Úbersicht 27: Organisation und Personal von CINCEUR/OMGUS, April 1949}

\section{CINCEUR}

Ofice of the Commander-in-Chief

Commander-in-Chief and Military Governor

Gen. $\quad$ Lucius D. Clay

Acting Political Adviser

Civ. James W. Riddleberger

Special Adviser

Civ. J. Anthony Panuch

Budget and Fiscal Director

Director of Management and Reports Branch, Chief

Col.

Stephen B.Elkins

Director of Intelligence

Civ. W. H.Schroder

Maj.Gen. William E.Hall

157 General Order Nr. 30 vom 31.7.1948.

158 General Order Nr. 46 und 47 vom 1.11 .1948$.

159 5/357-1/12; 5/357-1/14-15. Vgl. vor allem das Memorandum Sundquists vom 7.1.1949 (5/357$1 / 15)$

160 General Order Nr. 17 vom 9.6.1949. 


\author{
Inspector General \\ Director of Civilian Personnel \\ Secretary General (Acting)
}

Office of Military Government for Germany (US)

Commanding General and Deputy Military Governor

Chief of Staff

Deputy Chief of Staff

\section{Ofiice of the Secretary General}

Secretary General (Acting)

US Secretary, Bipartite Secretariat

US Secretary, Tripartite Secretariat (Prov)

US Secretary, Allied Secretariat

\section{Control Oficice}

Control Officer

Organization and Program Branch, Chief

Assistant Control Officer for Bipartite Affairs

Reports and Statistics Branch, Chief

Historical Branch, Chief

\section{Ofiice of the Adjutent General}

Adjutant General

Miscellaneous Branch, Chief

Military Personnel Branch, Chief

Reference Library, Chief

Travel Branch, Chief

Combined Travel Board, Chief

Publications Branch, Chief

Records Branch, Chief

Cable Control Branch, Chief

Message Control Branch, Chief

\section{Ofice of the Personnel Oficer}

Personnel Officer

Military Personnel Branch, Chief

Civilian Personnel Branch, Chief

\section{Ofiice of the Inspector General}

Inspector General

\section{Office of the Director of Intelligence}

Director

Deputy Director

Analysis and Research Branch, Chief

Plans and Policy Branch, Chief

EUCOM Requirements Branch, Chief

Security Branch, Chief

Liaison Branch, Chief

Special Project Branch, Chief

Administration Personnel Branch, Chief

Office of Director of Political Affairs

Director

Political Branch, Chief

Economics Branch, Chief

$\begin{array}{ll}\text { Maj.Gen. } & \text { G. J.Richards } \\ \text { Civ. } & \text { Robert M.Bamett } \\ \text { Civ. } & \text { Eric G. Gration }\end{array}$

Maj.Gen. George P.Hays

Brig.Gen. Charles K.Gailey

Civ. James E. King

Civ. Eric G. Gration

Maj. Walter E. Mather

Maj. Walter E.Mather

Maj. Gordon L. C.Scott

Civ. James L. Sundquist

Civ. Gordon P.Freese

Civ. Albert G.Sims

Civ. H. R. Habicht

Civ. David G. White

Lt.Col. George H.Garde

Maj. Herman S.Hoffman

Maj. Herman S. Hoffman

Civ. Henry A. Dunlap

Lt.Col. John B.Mallon

Civ. Jean J.Chenard

Civ. Monroe M.Richardson

Capt. Robert E. Reed

Civ. Charles R. Bakey

Capt. Sidway

Col. James T. Duke

Capt. J. J.Carollo

Civ. John E.Tromer

Col. Walter E. Jenkins

Col. Peter P.Rodes

Civ. Innis D. Harris

Civ. Innis D. Harris

Lt.Col. J. F. Eason

Civ. Donald H. Cooper

Civ. C. J.O'Connor

Civ. J. S. Arouet

Civ. Hans Kallman

WOJG RobertBaker

Civ. James W. Riddleberger

Civ. Perry Laukhuff

Civ. James W.Gantenbein 
Frankfun POLAD, Chief

Economics Branch Frankfurt. Chief

Foreign Representation Branch, Chief

Political Branch Frankfurt, Chief

Berlin Consular Branch, Chief

Frankfurt Consular Branch, Chief

Pablic Information Onice

Director

News Branch, Chief

German News Branch, Chief

Deputy Director for OMGUS PIO Frankfurt

BICO Branch, Chief

JEIA Branch, Chief

Oitice of the Economios Adviser

Economics Adviser

Deputy Economics Adviser

Food and Agriculture Group, Acting Chief

Industry Group, Acting Chief

Communications Group, Chief

Scientific Research Group, Chief

Transport Group, Acting Chief

Reports and Statistics Group, Chief

Omce of the Finmece Adviser

Acting Finance Adviser

Deputy of the Finance Adviser

Internal and External Finance Group, Acting Chief

Financial Intelligence Group. Chief

Central Bank Group, Chief

Foreign Exchange Depository Group, Acting Chief

Otice of Naval Adviser

Commander, US Naval Forces, Germany, and Naval Adviser

Aide. Chief of Staff, and Deputy Naval Adviser (USN)

\section{Armed Forces Division}

Director

Liaison Branch, Chief

Civil Aviation Branch, Chief

Meteorological Branch, Chief

\section{Civil Administration Division}

Director

Deputy Director for Operations

Consultant

Consultant

Tripartite Liaison Group, US Representation Alternate US Member

Deputy Director for Bizonal Affairs and Governmental Affairs Adviser

Secretariat and Reports Branch, Chief

Legislation Branch, Chief

Political Activities Branch, Chief

Population and Analysis Branch, Chief
Civ.

Civ.

Civ.

Civ.

Civ.

Civ.

Civ.

Civ.

Civ.

Civ.

Civ.

Civ.

Civ.

Civ.

Civ.

Civ.

Civ.

Civ.

Civ.

Civ.

Civ.

Civ.

Civ.

Civ.

Civ.

Rear Adm. John Wilkes

Capt. Joseph P. Thew

Maj.Gen. James P. Hodges

Lt.Col.

Civ.

Civ.

B. E.Steadman

Thomas D. Johnson

H. D.Perry

Civ.

Civ.

Civ.

Civ.

Civ.

Civ.

Civ.

Civ.

Civ.

Civ.

Civ.
William Bruce Lockling

iam Schott

A ndelin

Marshall M. Vance

William R. Haggard

Hyman Chamiak

Lawrence Wilkinson

Philip Hawkins

Philip M. Raup

Frank L. Mayer

Eugene H.Merrill

C. H. Nordstrom

W. T. Cantrell

C. K. Nichols

Jo Fischer Freeman (vacant)

Ralph McCabe

Emil Lang

Henry C. Conrad

Frank J. Roberts
Roy I. Kimmel

Stephen Freeland
Edward H.Litchfield

H. Philip Mettger

Lawrence E. DeNeufville

Harold Landin

Edward H. Litchfield

Hans Simons

Kenneth A. Dayton

AlexanderForest

Leslie Grant

John Elliot

Karl A.Shafer 
Civil Service and Administrative Courts Branch, Chief Civ.

Public Safety Branch, Chief Civ.

Public Health Branch, Chief Col.

Public Welfare Branch, Chief Civ.

Displaced Persons Branch, Chief Civ.

Civil Liberties and Democratization Branch, Chief Civ.

\section{Education and Cultural Relations Division}

Director

Deputy Director

Research and Planning Branch, Chief

Cultural Affairs Branch, Chief

Education Branch, Chief

Community Education Branch, Chief

Religious Affairs Branch, Chief

\section{Information Services Division}

Director

Deputy Director

Policy and Programming Branch, Chief

Fiscal and Budget Control Branch, Chief

Opinion Surveys Branch, Chief

Editorial Projection Branch. Chief

US Feature Service Branch, Chief

Information Centers and Exhibition Branch, Chief

Motion Picture Branch, Chief

Radio Branch, Chief

Press Branch, Chief

Publications Branch, Chief

Acting Cooperations Coordinator

Publishing Operations Branch, Chief

\section{Legel Division}

Director

Associate Director

Legal Advice Branch, Chief

Legislation Branch, Chief

Administration of Justice Branch, Chief

Prisons Branch, Chief

\section{Manpower Division}

Director

Labor Information and Orientation Branch, Chief

Labor Relations Branch, Chief

Research and Analysis Branch, Chief

\section{Property Division}

Director

Deputy Director

Reparations and Restitution Branch, Chief

Property Control and External Assets Branch, Chief

Central Filing Agency, Chief

\section{Military Tribunaks (Nümberg)}

Executive Presiding Jugde, Justice

Civ.

Civ.

Civ.

Civ.

Civ.

Civ.

Civ.

Col.

Civ.

Civ.

Civ.

Civ.

Civ.

Civ.

Civ.

Civ.

Civ.

Civ.

Civ.

Civ.

Civ.

Col.

Civ.

Civ.

Civ.

Civ.

Civ.

Civ.

Civ.

Civ.

Civ.

Civ.

Civ.

Civ.

Civ.

Civ.

Civ.

Military Tribunal IV (Ministries Case) Presiding Justice
Ellsworth D.Wolfsperger

Theo E. Hall

Milford T. Kubin

Mildred B.Smith

L. G. Kelly

David R. Hunter

Alonzo G. Grace

Milton E.Muelder

Wallace Strevell

Ralph A. Burns

John O. Riedl

Lawrence E. Norrie

C. Arild Olsen

Gordon E. Textor

Thomas P. Headen

Alfred V. Boemer

Daniel C.Mulloney

Leo P. Crespi

John M. Stuart

Charles B. Arnot

Patricia Van Delden

Arthur Mayer

Charles S. Lewis

Arthur D. Eggleston

Lawrence P.Dalcher

Thomas J.Crowell

Kendall Foss

John M. Raymond

William E. McCurdy

James E. Heath

John J. Barron

Mortimer Kollender

Sidney H. Souter

Leo R. Werts

Nels Anderson

John K. Meskimen

Albert H. Berman

Philip Hawkins

E. J.Cassoday

Orren R.McJunkins

Fred E. Hartzsch

Bernard Fischbein

William C.Christiansen William C.Christiansen 
Secretary Generd for Military Tribunals (Nürnberg)

Secretary General of Tribunals

Defense Center, Director

Language Division, Director

Civ.

Capt.

Civ.

Civ.

Howard H. Russell

Lowell O. Rice

Court Archives, Chief

Hans E. Gleichman

Barbara S. Mandellaub

Omice of Chief of Counsel for War Crimes (Nürmberg)

Chief of Counsel

Document Disposal Division, Director

Economics Ministries Division (Deputy Chief of Counsel), Director

Political Ministries Division (Deputy Chief of Counsel). Civ.

Publications Division (Deputy Chief of Counsel), Civ.

Director

Publications Division (German Edition), Director

Public Relations Division and Public Information

Division, Director

Reproduction Division, Director

Brig.Gen. Telford C. Taylor

Civ. Fred Niebergall

Civ. Morris Amchan

Robert M.W. Kempner

Drexel A.Sprecher

$\begin{array}{ll}\text { Civ. } & \text { Paul H. Gantt } \\ \text { Civ. } & \text { Eugene Philips }\end{array}$

Civ. Erich G. R. Rehberg

US Military Government Courts (Nürnberg)

Courts of Appeals

Office of the Chief Attomey (Nürberg)

Chief Attorney

Civ.

Worth B. McCauley

Secretary General for Courts Administration (Nürnberg)

Sectetary General

Civ.

Howard H. Russell

Board of Review (Nümberg

President

Civ.

Johnson T. Crawford

Bipartite Boand

US Member

Gen.

Lucius D.Clay

Bipartite Secretariat

US Secretary

Maj.

Walter E. Mather

Ofice of US Chairman, Bipartite Control Ofice

Chairman

Deputy Chairman

Maj.Gen. Clarence L. Adcock

Civ. Robert K. Phelps

US Secretariat, Chief

Civ.

Ernest Linde

ERP Secretariat (US Element), Chief

Management and Budget Office, Chief

Lt.Col.

E. H. Harrelson

Reports Branch

Administrative Services Branch, Chief

Civ.

E. L. MacDonald

Civ.

C. B. Millican

Office of the Adjutant General, Adjutant General

Civ.

R. E. Brant

Lt.Col. G. M. Weir

\section{Commerce and Industry Group}

Chief (US)

Civ.

L. E. Spencer

Industry and Production Branch, Chief

Civ.

A. S. Radford

Fuel and Power Branch, Chief

Civ.

Plans and Allocations Branch, Chief

Civ.

F. K. McQueary

Research and Statistics Branch,

K. F. Bode

OMGSTEG Branch, Chief

Dewilda Naramore

Civ.

J. C. Cunningham

Food, Agriculture and Forestry Group

Chief (US)

Civ.

Stanley Andrews

Allied Administration Branch, Chief

Civ.

R. C. Nichols

Food Planning and Statistics Branch, Chief

Civ.

B. A.Cash-Reed 
Food Supply Branch. Chief

Forestry Branch, Chief

\section{Transport Group}

Chief (UK)

Deputy Chief (US)

Secretariat and Reports Branch, Chief

Highway and Highway Transport Branch, Chief

Inland Water Transport Branch, Chief

Maritime Ports and Shipping Branch. Chief

Movements Branch, Chief

Plans and Intemational Affairs Branch, Chief

Rail Branch, Chief

\section{Communications Group}

Chief (UK)

Deputy Chief (US)

Finance Branch, Chief

Posts and Personnel Branch, Chief

Reports and Statistics Branch, Chief

Telecommunications Branch, Chief

\section{Finance Group}

Chief (UK)

Deputy Chief (US)

Financial Policy and Institutions Branch, Chief

Public Finance Branch, Chief

\section{Manpower Group}

Chief (UK)

Deputy Chief (US)

Labor Relations Branch. Chief

Labor Allocations Branch, Chief

Reports and Statistics Branch, Chief

Social Insurance Branch, Chief

Wages and Labor Standards Branch, Chief

\section{Civil Service Group}

Chief (US)

Decartelization Commiscion (US Element)

Chief

\section{Legal Group}

Chief (US)

\section{Bipartite Statistical Office}

Chief (UK)

Deputy Chief (US)

\section{US Customs Group}

Chief

\section{Central Bank Group}

Office of the Financial Adviser, (US), Chief

\section{Combined Coal Control Group}

US Chairman

Production Branch, Chief

$\begin{array}{ll}\text { Civ. } & \begin{array}{l}\text { G. Gamett } \\ \text { Civ. }\end{array} \\ \text { J. C. Kircher } \\ \text { Civ. } & \text { Sir Robert Inglis } \\ \text { Civ. } & \text { W. A. Fagan } \\ \text { Civ. } & \text { F. J.Fallin } \\ \text { Civ. } & \text { E. B.Sutton } \\ \text { Civ. } & \text { H. Calvert } \\ \text { Civ. } & \text { G. H.McNeill } \\ \text { Civ. } & \text { B. S. Lloyd } \\ \text { Civ. } & \text { A. A. Earley } \\ \text { Civ. } & \text { H. G.Warvel } \\ & \\ \text { Civ. } & \text { L. G.Semple } \\ \text { Civ. } & \text { J. W. Campbell } \\ \text { Civ. } & \text { W. L.Slattery } \\ \text { Civ. } & \text { P. R. J.Marr } \\ \text { Civ. } & \text { R. Ransier } \\ \text { Civ. } & \text { A. J.Hinckley }\end{array}$

Civ.

Civ.

Civ.

Civ.

Joseph Charles Brommage Howard P. Jones

H. C. F. Holgate

A. M. Hillhouse

Civ.

Civ.

G. W. J.Cole

S. G. Wynn

C. S. Lovell

E. C. M.Cullingford

E. L. Deuss

T. J.Beatty

J. L. Harmon

Civ.

Ellsworth D.Wolfsperger

Civ.

Richard Bronson

Civ.

Richard D. Kearney

Civ.

Civ.

E. H. Slade

H. H. Lee

Civ.

J. Miles

Civ.

Henry C.Conrad

Civ.

Civ.

W. J.German

A. F. Marshall 
Joim Foreign Exchange Agency

US Member

Civ.

$\begin{array}{ll}\text { Civ. } & \text { Jo Fisher Freeman } \\ \text { Civ. } & \text { Sir Eric Coates } \\ \text { Civ. } & \text { P. L. Beaulieu }\end{array}$

Allied Bank Conmission

Acting US Member

UK Member

French Member

Mitiny Security Board

Commissioner

Deputy Commissioner

Military Division, Chief

Industrial Division, Chief

Scientific Research Division, Chief

Jolut Export-Import Agency (Franlfurt)

Director General

Deputy Director General

Deputy Director General

Administrative Branch, Chief

US Secretariat, Chief

UK Secretariat, Chief

French Secretariat, Chief

Legal Office, Chief

Reports and Statistics Office, Chief

Ofice of the Comptroller

Comptroller

Ofice of Forelgen Trade

Director

Oritice of the Director of Openations

Director of Operations

$\begin{array}{ll}\text { Maj.Gen. } & \text { James P. Hodges (USAF) } \\ \text { Civ. } & \text { Earl F. Thomson } \\ \text { Lt.Col. } & \text { Gien W. Campbell } \\ \text { Civ. } & \text { Frank L. Mayer } \\ \text { Civ. } & \text { C. H. Nordstrom }\end{array}$

Civ. William John Logan

Civ. John F. Cahan

Civ. P.Amal

Civ. F. Barker

Civ. B. R.Thorne

Civ. T. H.Cox

Civ. R. F. Fougere

Civ. C.Denney

Civ. H. P.Dawson

Civ. Moore C. McIntosh

Civ. C. E. Bingham

Civ. M. S. Verner

\section{Der Übergang von OMGUS auf HICOG}

Entscheidend für die verwaltungsgeschichtliche Entwicklung von OMGUS im Jahre 1949 waren zwei Tatsachen:

Zum einen hatten Clay und seine Mitarbeiter in der amerikanischen Armee seit 1945 stets den Wunsch, ihre Verantwortung für die Verwaltung des besetzten Deutschland möglichst bald in zivile Hände, also in die Zuständigkeit des State Department, zu ubertragen. Aus einer Reihe von Gründen waren diese Bemühungen jedoch lange erfolglos. Am 8. Januar 1948 kündigte der amerikanische Außenminister George Marshall vor dem außenpolitischen AusschuB des Senats an, daB nach seiner Auffassung die Verwaltung des besetzten Deutschland bis Jahresmitte in die Verantwortung des State Department übergehen könne. ${ }^{161}$ Daraufhin wurde in Washington ein gemeinsamer Ausschuß von State und War Department gebildet: Das State-Army-Committee on Transfer (SACOT). In Deutschland führte diese Entwicklung zu eifriger Tätigkeit vor allem des Control Officer, James L. Sundquist, der bereits am 14. Januar 1948 General Clay ein entsprechendes Memorandum fertigte. Am 2. März 1948 kündigte General Clay seinerseits die Errichtung eines besonderen Ausschusses bei OMGUS an,

161 Clay: Entscheidung in Deutschland, S. 433 ff.; Zink: The United States in Germany, S. 44. 
des Committee on State Army Reorganization (COSAR). Dieser Ausschuß war für den Bereich des Commander-in-Chief in Europe ausschließlich für die Vorbereitung des Übergangs der Besatzungsverwaltung auf eine zivile Stelle verantwortlich. Die Einzelmaßnahmen wurden zum großen Teil im Control Office, OMGUS, getroffen. ${ }^{162}$

Zum anderen war es nach dem ergebnislosen Ausgang der Londoner Außenministerkonferenz vom Dezember 1947 und der Funktionsunfähigkeit des Kontrollrats seit Frühjahr 1948 im Zusammenhang mit der Blockade Berlins klar geworden, daß eine einvernehmliche Regelung der Deutschlandfrage durch alle vier Besatzungsmächte nicht mehr in Betracht kommen konnte. Auf der Londoner Sechs-Mächte-Konferenz, die bis Anfang Juni 1948 tagte, wurden die Voraussetzungen für die Vereinigung der drei westlichen Besatzungszonen zu einem (west)deutschen Staat geschaffen. ${ }^{163}$ OMGUS reagierte auf die Ergebnisse dieser Konferenz, indem es den amerikanischen Anteil eines Drei-Mächte-Ausschusses errichtete: das United States Element eines Committee on Tripartite Military Government Organization. ${ }^{164}$ Dieser und andere vergleichbare Drei-Mächte-Ausschüsse bereiteten auf alliierter Seite die Errichtung der künftigen Bundesrepublik Deutschland vor. An der Spitze dieser Tripartite Organization stand ein Tripartite Board, der aus den drei westlichen Zonenbefehlshabern bestand. ${ }^{165}$ Parallel zu dieser Zusammenarbeit erfolgte die Aufnahme französischer Vertreter in Besatzungsbehörden, die vorher von Briten und Amerikanern gemeinsam geführt wurden: der Military Security Board, der Combined Travel Board, die Allied Bank Commission, die Combined Coal Control Group, die Combined Steel Control Group und die Joint Export Import Agency. SchlieBlich wurde ein französischer Verbindungsstab beim Bipartite Control Office errichtet.

Erst im April 1949 einigten sich die Außenminister der drei Westmächte in Washington, in welcher Form sie ihre Aufsicht uber eine künftige westdeutsche Zentralregierung führen wollten. Im Übereinkommen über den Zusammenschluß der drei Zonen war eine Allied High Commission vorgesehen, in der jeweils ein Hoher Kommissar der drei Besatzungsmächte oder sein Beauftragter vertreten sein sollte, um die nach wie vor als erforderlich angesehene Kontrolle einer künftigen (west)deutschen Zentralregierung zu übernehmen. Rechtliche Grundlage der Drei-Mächte-Kontrolle sollte ein Besatzungsstatut werden. ${ }^{166}$ Bei OMGUS ging man damals davon aus, daß die oben genannten Drei-Mächte-Einrichtungen in dieser oder jener Form auch unter den Hohen Kommissaren fortbestehen sollten. Auf Landesebene war allerdings nur ein kleiner Stab vorgesehen, der vor allem für die Überwachung der Gesetzgebung der Länder zuständig sein sollte. Insgesamt erschien ein Personalbedarf von etwa 800 Bediensteten völlig ausreichend. Im Mai 1949 schuf man in Washington einen gemeinsamen Ausschuß des State Department, des War Department und der Verwaltung für europäische Zusammenarbeit (OEEC): das Committee on Planning for Germany (CPG). ${ }^{167}$

Am 15. Mai 1949 kehrte General Lucius D. Clay in die Vereinigten Staaten zurück; drei Tage später wurde bekanntgegeben, daB John J.McCloy als amerikanischer Hoher Kommissar vorgesehen sei. Anfang Juni 1949 erhielten die verantwortlichen

\footnotetext{
$162 \mathrm{Vgl}$. die Monthly Reports of the Military Govemor 1949 und die Akten des Control Office, insbesondere 5/361-2/30.

163 Benz: Von der Besatzungsherrschaft, S.156f.

164 Zink: The United States in Germany, S. 45.

165 Vgl. Vogel: Westdeutschland 1, S.40f.

166 Erdmann: Zeit der Weltkriege, S. 774f.

167 Monthly Reports of the Military Governor 1949.
} 


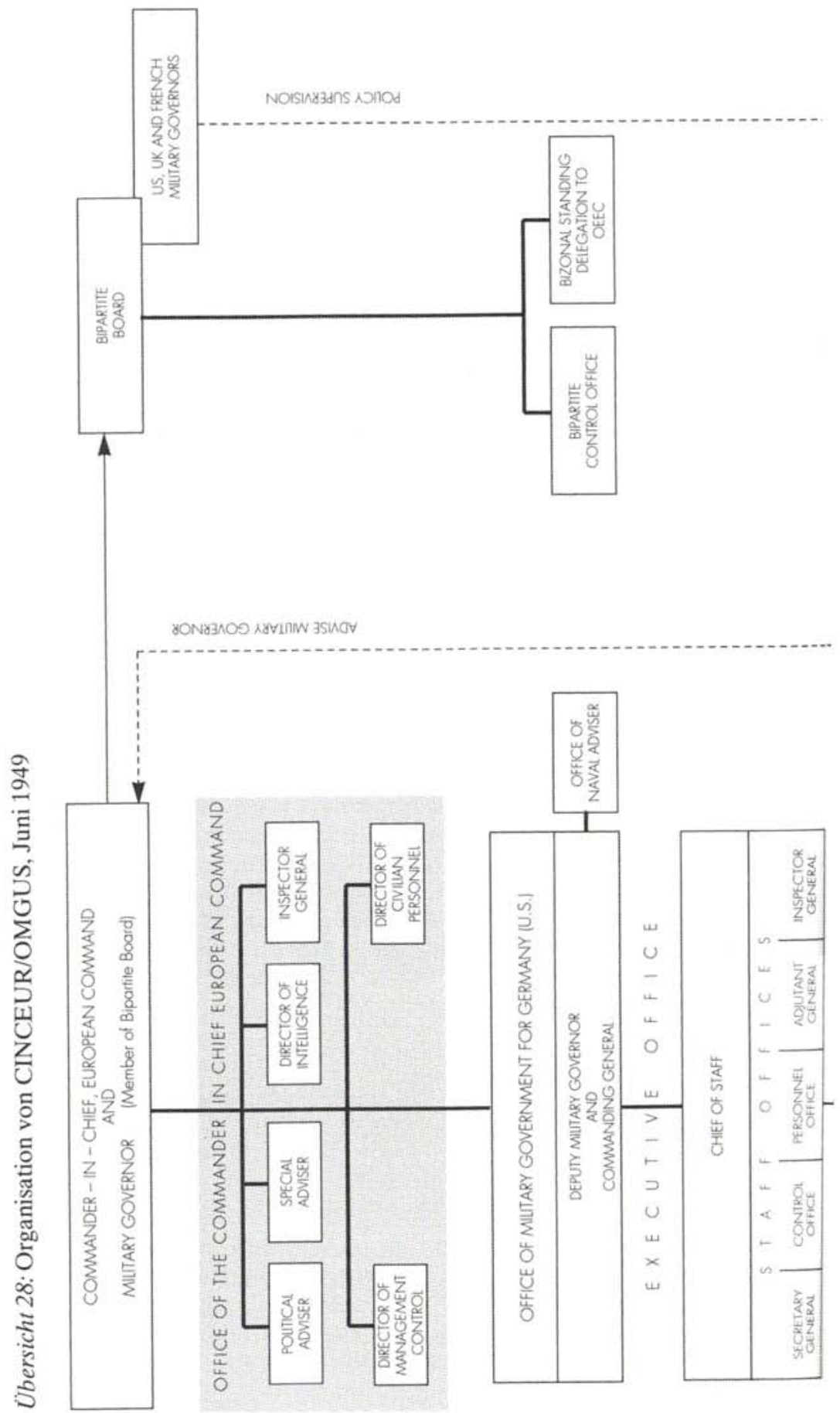




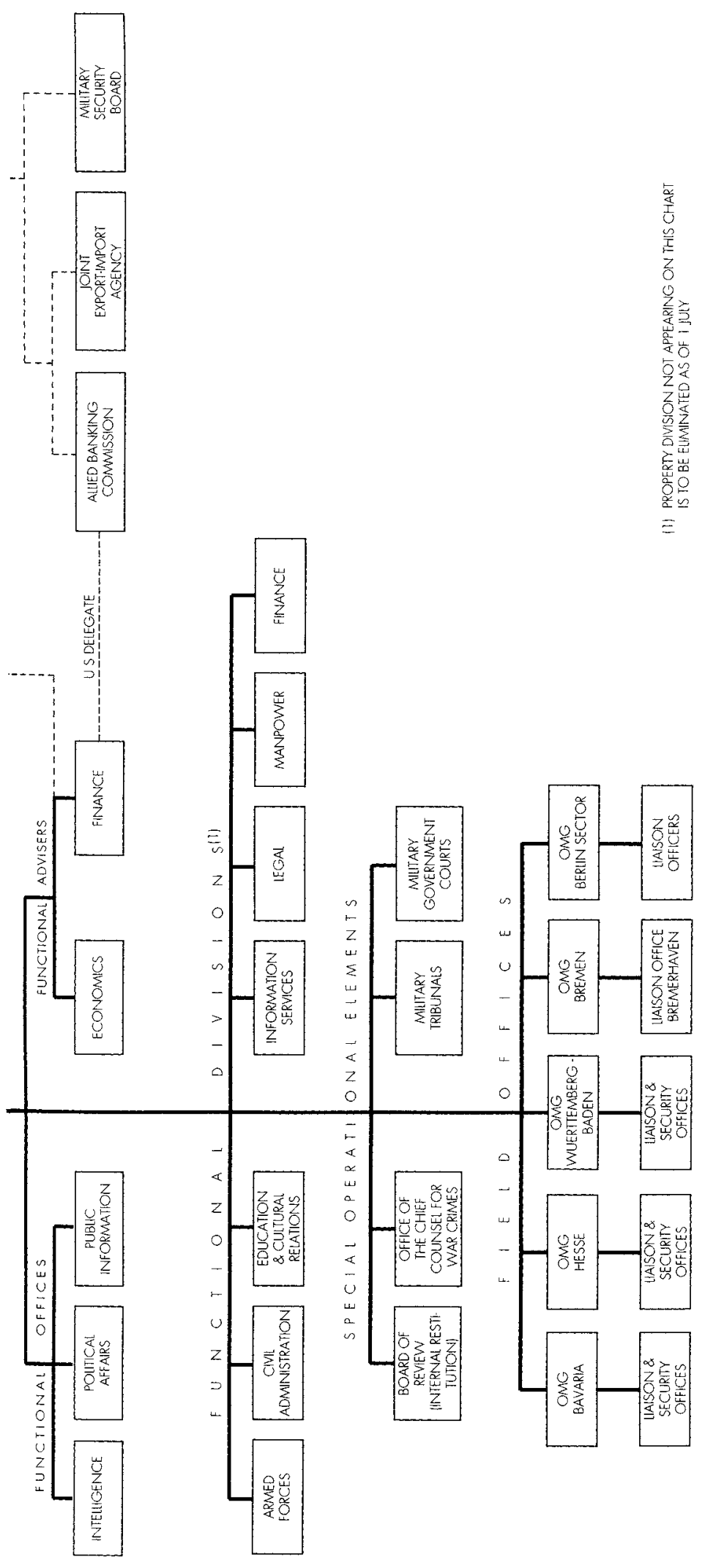




\section{Übersicht 29: Organisation von HICOG, September 1949}

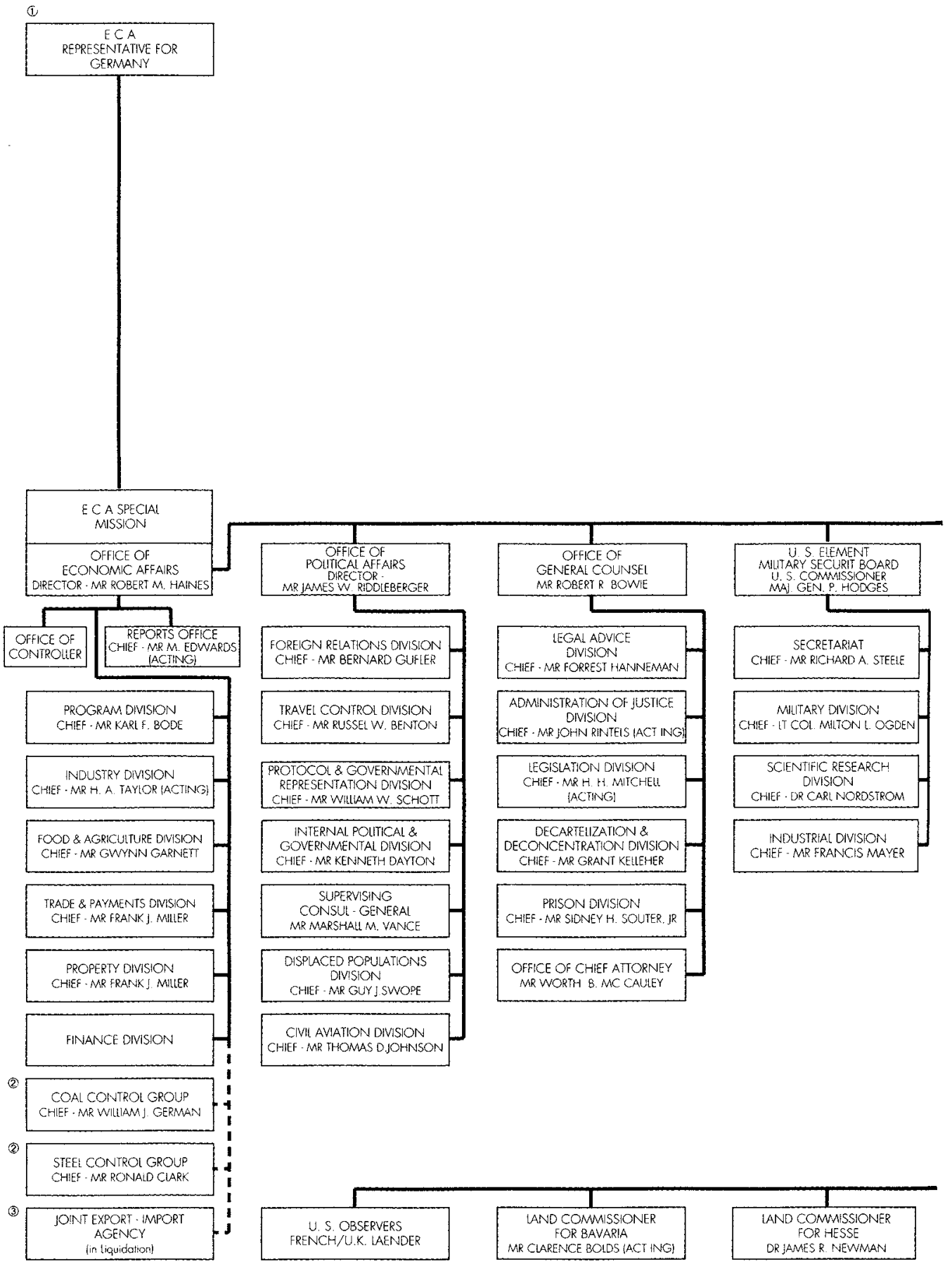




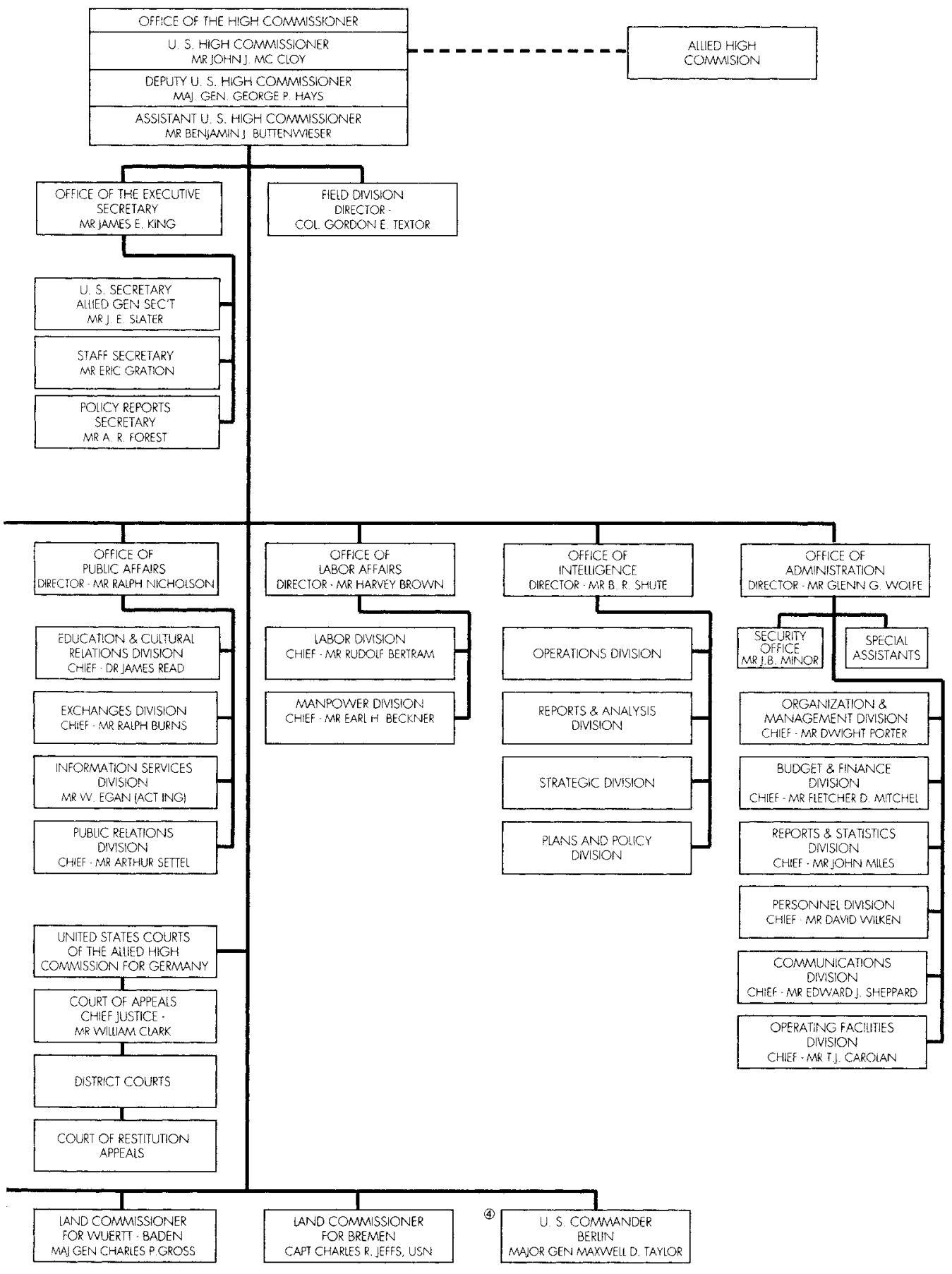

(1) ECA REPRESENTATIVE FOR GERMANY - BY EXECUTIVE OROER. MR JOHN MC CIOY AS US HIGH CONMISSIONER FOR GERMANY, ALSO SERVES AS ECA REPRESENTATIVE FOR GERMANY, LINDER THE IMMEDIATE SUPERVISION OF THE ADMINISTRATION FOR ECONOMIC COOPERATION \& IN COORDTINATION WITH THE SPECIAL REPRESENTATIVE FOR GUROPE [SUJJEECT HOWEVER TO CONSUITATION WITH AND ULTIMATE DIRECTION BY THE PRESIDENT].

(2) US ELEMENTS, COAL CONTROI GROUP AND STEEL CONTROL GROUP, RECEIV POICY GUIDANCE FROM DIRECTOR OF ECONOMC AFFAIRS, FOR TRIPARTITE ACTIVIIES, THE GROJPS ARE RESPONSIBLE TO THE ECONOMCS COMMTTEE, ALLIEO HIGH COMMISSION.

(3) US ELEMENT RECENES POICY GUIDANCE FROM DIRECIOR ECONOMIC AFFAIRES, AGENCY IS ADMINISTRATMELY RESPONSIBIE TO FOREIGN TRADE AND EXCHANCHE COM MITEE. ALLLD HIGH COMMISSLN, DURING IOUUDATION

(4) THE US COMMANDER, BERIIN SERVES AS THE REPRESENTATIVE IN BERLIN OF THE US HIGH COMMISSIONER FOR GERMANY, FOR MHUTARY MATERS HE REPORTS TO THE COM. MANDER - IN - CHIEF, EUROPEAN COMMAND. 
Mitarbeiter von OMGUS in Washington Gelegenheit, ihre Auffassung über die künftige Entwicklung in Deutschland im einzelnen vorzutragen. Am 1.Juli 1949 begab sich der durch Executive Order Präsident Trumans vom 6. Juni 1949 zum United States High Commissioner ernannte McCloy ${ }^{168}$ nach Deutschland; der Schwerpunkt der Planungen für die Überleitung der Besatzungsverwaltung in zivile Hände verlagerte sich damit auf die andere Seite des Atlantik.

Am 2. Juli 1949 gab der Hohe Kommissar für Deutschland, John J. McCloy, bekannt, daß er die Aufgaben eines Militärgouverneurs für die amerikanische Besatzungszone übernommen habe; dies bedeutete, daB er seit diesem Tage die Funktionen eines Militärgouverneurs und eines Hohen Kommissars gemeinsam wahrnahm. Er führte nunmehr die Amtsbezeichnung: US High Commissioner for Germany, Military Governor, US Zone Germany. ${ }^{169}$

Durch General Order Nr.26 vom 19.Juli 1949 wurde aus der Civilian Personnel Branch das Personnel Office, aus der Budget and Fiscal Branch und der Organization and Program Branch des Control Office sowie dem Administrative Office im Office of Political Affairs ein Provisional Office of Administration, OMGUS. Dieses provisorische OMGUS-Amt diente ebenso provisorisch dem US High Commissioner und war insoweit für die verwaltungsmäßige Abwicklung des Übergangs der Regierungsfunktionen in Deutschland von der amerikanischen Armee (War Department) auf den zivilen Dienst (State Department) verantwortlich. Durch General Order Nr. 27 vom 26. Juli 1949 billigte McCloy den ihm vorgelegten, nunmehr vollständigen Organisationsplan des Office of the US High Commissioner for Germany. Diese Behörde gliederte sich im Unterschied zu OMGUS nicht in Abteilungen (Divisions), sondern in Ämter (Offices). Neben dem Office of Economic Affairs, dem im Rahmen der europäischen wirtschaftlichen Zusammenarbeit besondere Bedeutung zukam, gab es sechs weitere Ämter und den amerikanischen Anteil an der Drei-Mächte-Einrichtung des Military Security Board. Diese Struktur ist insgesamt mit dem Aufbau von OMGUS kaum noch zu vergleichen. Wie in amerikanischen Zivilbehörden üblich, wurden die acht Offices - als solches ist auch der amerikanische Anteil des Military Security Board anzusehen - grundsätzlich in zwei bis sechs bzw. neun Divisions gegliedert. Der Schwerpunkt der Tätigkeit von HICOG lag eher im politischen, weniger im wirtschaftlichen Bereich. Mit Ablauf des 14. August 1949 wurde das Hauptquartier des Office of Military Government for Germany unter gleichzeitiger Auflösung des Office of the Chief of Staff, dessen Funktionen auf das Provisional Office of Administration übergingen, nach Frankfurt am Main verlegt. ${ }^{170}$ Unter OMGUS, Berlin, waren de iure et de facto nunmehr lediglich die Teile der Militärverwaltung zu verstehen, die - abgesehen vom Office of Military Government for Berlin Sector - vorübergehend noch in Berlin ihren Dienstsitz hatten.

Die Angleichung der Organisationsstruktur von OMGUS und HICOG, die gleichzeitig als Vorbereitung der Auflösung von OMGUS anzusehen ist, wird durch drei am 3. September 1949 erlassene General Orders sinnfällig. ${ }^{171}$ So wurden die Information Services Division, die Education and Cultural Relations Division und das Public Information Office zum Provisional Office of Public Affairs, OMGUS, vereinigt, die Legal Division in das Provisional Office of General Counsel, OMGUS, überführt und schlieBlich die Ämter des Economics und Finance Adviser sowie die Finance Division

\footnotetext{
168 Zink: The United States in Germany, S. 45.

169 General Order Nr. 22 vom 2.7.1949.

170 General Order Nr. 36 vom 12.8.1949.

171 General Orders Nr. 44 - 46 vom 3.9.1949.
} 
zum Provisional Office of Economic Affairs, OMGUS, zusammengefaßt. Diese Struktur von OMGUS-Verwaltungseinheiten entsprach dem bereits genehmigten Organisationsplan von HICOG.

Am 21. September 1949 trat das Besatzungsstatut für (West)Deutschland bzw. die Bundesrepublik Deutschland in Kraft, in dem es u. a. hieß: „Die deutschen Behörden sollen im allgemeinen die Freiheit haben, administrativ und legislativ tätig zu werden“, ${ }^{172}$ sofern keine alliierten Vorbehalte im Wege standen. Damit war für die drei westlichen Militärgouverneure auch formal kein Raum mehr; sie wurden an diesem Tage förmlich durch die Hohen Kommissare ersetzt, die zusammen die Alliierte Hohe Kommission für (die Bundesrepublik) Deutschland bildeten. Das Office of Military Government for Germany (US) bestand ab 21. September 1949 nicht mehr.

172 Schmid: Deutschlands Weg, S. 365. 


\section{B. Systematische Übersicht Einzelne Verwaltungseinheiten der amerikanischen Militärregierung in Deutschland}

\section{Die Spitzengliederung im Allgemeinen ${ }^{1}$}

Vorläufer des Office of Military Government for Germany (US) (OMGUS) war die durch General Order Nr. 80, ETO, vom 9. August 1944 in Bushy Park bei London errichtete US Group Control Council (USGCC) (Germany). Oberhalb der Ebene der Fachämter (Functional Offices) und der Fachabteilungen (Functional Divisions) wies sie im Frühsommer 1945 in Deutschland die für amerikanische militärische Kommandobehörden übliche Struktur auf. Unter dem Military Governor und Oberbefehlshaber für das European Theater of Operations United States Army (ETOUSA), General Dwight D. Eisenhower, stand der Deputy Military Governor, Germany, and Commanding General, USGCC, Lt.Gen. Lucius D. Clay, an der Spitze der Militärregierung.

Clay und seine unmittelbaren Mitarbeiter bildeten das Executive Office (Office of the Deputy Military Governor). Zu seinen engsten Mitarbeitern zählten drei Assistant Deputies, die sich nach sachlichen Gesichtspunkten die Aufsicht uber die Fachabteilungen teilten: Der Assistant Deputy for Resources, Brig.Gen. William H. Draper, der Assistant Deputy for Trade and Finance, Laird Bell, und der Assistant Deputy for Public Services, Maj.Gen. Oliver P. Echols. Unmittelbar dem Deputy Military Governor zugeordnet waren zwei Berater (Advisers), die keinerlei unmittelbare Weisungsbefugnisse gegenüber nachgeordneten Stellen der Militärregierung hatten: der Political Adviser, Ambassador Robert D. Murphy, und der Legal Adviser, Charles Fahy. Die Besetzung des Executive Office wurde vervollständigt durch den Chief of Staff, damals Brig.Gen. Bryan L. Milburn, der für die üblichen Stabsdienste verantwortlich war. Seit dem 24. Juli 1945 bekleidete der bisherige Assistant Deputy for Public Services, Maj.Gen. Oliver P. Echols, die Position eines Assistant Deputy Military Governor mit eigenem Office. Deputy for Public Services wurde Brig.Gen. Frank C. Meade. Dem Executive Office waren zwei Ausschüsse, das Executive Committee und das Demilitarization Committee, zugeordnet.

Das Executive Committee bestand unter dem Vorsitz des Deputy Military Governor aus den Mitgliedern des Executive Office und dem Director of Intelligence, einem von vier Leitern der nachgeordneten Fachämter. Das Demilitarization Committee setzte sich ebenfalls unter dem Vorsitz des Deputy Military Governor aus den Leitern der drei Fachabteilungen für Heer, Marine und Luftwaffe sowie dem Stabschef zusammen. Zum Leitungsbereich gehörten der Secretary General (Generalsekretär), Col. T.

${ }^{1}$ Die Beschreibungen stützen sich u. a. auf die Provenance Descriptions, die während des OMGUS-Projektes in den Jahren 1977 bis 1980 vor allem von Heiner Adamsen (Wuppertal), Brewster S. Chamberlin (Washington, D.C.), Michael Fichter (Berlin), Hartmut Hagner, Josef Henke und Klaus Oldenhage (alle Koblenz) und Jürgen Wetzel (Berlin) für die OMGUS-Ebene gefertigt wurden. Hinzukommt die Serie der General Orders 1945 bis 1949 (BA Z 45 F OMGUS, bzw. IfZ-Archiv MA 1417) und die Mikrofiches zu den Akten vor allem des Control Office, OMGUS, und des Adjutant General, OMGUS, sowie die in diesem Beitrag abgedruckten Organisations- und Personalübersichten. Vgl. auch J. Henke: Economic Reconstruction, S. $472 \mathrm{ff}$. 
W. Hammond (seit 1.September 1945 Col. David Marcus), und der Director of Administrative Services, Col. James B. Edmunds, die für fachneutrale Aufgaben - nicht nur für die Dienststellenverwaltung - als Leiter eigener Offices verantwortlich waren. Zu den Mitarbeitern des Director of Administrative Services zählten u. a. der Adjutant General, Lt.Col. James L. Williams (ab 4. September 1945 Lt.Col. F. W. Marshall), und der Personnel Officer, die später an der Spitze eigener Staff Offices standen.

Lucius D. Clay behielt die Position des Deputy Military Governor und Commanding General, USGCC bzw. OMGUS, unter Eisenhower und - ab 30. November 1945 - unter dessen Nachfolger General Joseph T. McNarney - bis zum 15. März 1947, als er selbst Nachfolger McNarneys wurde.

Als Commander-in-Chief, European Command (CINCEUR), and Military Governor übte Clay dann eine doppelte Befehlsgewalt aus, indem sowohl das Headquarters, European Command, in Frankfurt und Heidelberg, damit die amerikanischen Truppen, als auch das von diesem Hauptquartier im übrigen völlig unabhängige Office of Military Government for Germany (US) seinen Weisungen unterworfen waren. Clay bediente sich für diese Zwecke eines eigenen Office of the Commander-in-Chief, European Command, das neben mehreren Advisers und Assistants vier Verwaltungseinheiten zur Erledigung fachneutraler Aufgaben umfaBte. An der Spitze dieser vier Einheiten standen der Budget and Fiscal Director, der Director of Management Control, der Director of Intelligence, der Inspector General und der Director of Civilian Personnel. Diese Zusammensetzung blieb im ganzen bis zur Rúckkehr Clays in die Vereinigten Staaten von Amerika im Mai 1949 konstant.

Nachfolger Clays wurde der Diplomat John J. McCloy, der vom 2. Juli bis 21. September 1949 als United States High Commissioner for Germany und als Military Governor for the United States Zone, Germany, amtierte. Nachfolger Clays als Deputy Military Governor und Commanding General of the Office of Military Government for Germany (US) wurde am 15. Mărz 1947 Maj.Gen. Frank A. Keating, der seit dem 15. Oktober 1946 Assistant Deputy Military Governor for Operations und Deputy Commanding General, OMGUS, gewesen war. Mit Wirkung vom 30 . August 1947 trat Maj.Gen. George P. Hays bereits an die Stelle Keatings als Deputy Military Governor und übte dieses Amt bis zur Auflösung von OMGUS im September 1949 aus.

Die besondere Rolle, die die Assistant Deputies und Advisers in der Spitzengliederung der Militärregierung spielten, rechtfertigt eine Darstellung der Personalentwicklung in diesem Bereich. Brig.Gen. Bryan L. Milburn wurde durch General Order Nr.3 vom 25. November 1944 zum Chief Coordinator of Planning und durch General Order Nr. 2 vom 7. Mărz 1945 zum Assistant Acting Deputy, Headquarters USGCC, ernannt. Im Juni 1945 wurde die Spitzengliederung - wie geschildert - insoweit neu organisiert, als drei Assistant Deputies im Executive Office eingesetzt und Bryan L. Milburn zum Chief of Staff ernannt wurde. Im Juli desselben Jahres schuf man die Position eines Assistant Deputy Military Governor und besetzte sie mit Maj.Gen. Oliver P. Echols, dessen Nachfolger als Assistant Deputy for Public Services Brig.Gen. Frank C. Meade wurde. Mit der Umwandlung von USGCC in OMGUS wurden die drei Assistant Deputies für die Beaufsichtigung bestimmter Fachbereiche abgeschafft, so daB nur der Assistant Deputy Military Governor im Amt blieb, der im Laufe des Jahres 1946 in den Quellen auch als Assistant Deputy Military Governor for Operations bezeichnet wurde. Nachfolger Echols' wurde Anfang April 1946 Maj.Gen. Clarence L. Adcock mit der Amtsbezeichnung Assistant Deputy Military Governor for Operations und Deputy Commanding General, OMGUS. Ihm folgten mit Wirkung vom 15. Oktober 1946 Maj.Gen. Frank A. Keating, seit 15. März 1947 Brig.Gen. Cornelius E. Ryan, der die Amtsbezeichnung Assistant to the Deputy Military Governor, OMGUS, führte 
und gleichzeitig amerikanisches Mitglied in der Kommandatura in Berlin wurde. In beiden Positionen folgte ihm am 10. September 1947 Brig.Gen. William Hesketh, der bis Anfang 1948 im Amt blieb. In späteren Organisationsübersichten wird diese Position nicht mehr genannt.

Der Begriff Adviser/Advisor ${ }^{2}$ taucht in den Quellen der amerikanischen Militärregierung in mehrfacher Bedeutung auf. Anfangs gab es persönliche Fachberater des Stellvertretenden Militärgouverneurs, die aufgrund dieser Funktion keinerlei Weisungsbefugnisse gegenüber den Functional Offices oder Functional Divisions besaßen. Der bedeutendste unter ihnen war der Political Adviser, Botschafter Robert D. Murphy, der erst 1949 von James Riddleberger abgelöst wurde. Es kam aber durchaus auch vor, daB einzelne dieser Advisers - wie der Economics, Finance oder Legal Adviser - in Personalunion Leiter einer fachlich korrespondierenden Abteilung waren. Der Naval Adviser verfügte dagegen stets über ein eigenes Office.

Die zweite Gattung von Beratern tat ihren Dienst ab 15. Mai 1947 als Sonderberater Clays im Office of the Commander-in-Chief, European Command. Damals gab es neben einem Special Adviser, einen Political, einen Economics und einen Finance Adviser, die freilich nicht mehr zu OMGUS, sondern wie Clay zu EUCOM gehörten. Ab März 1948 veränderte sich das Bild bei OMGUS noch dadurch, daß die Economics Division, OMGUS, und die Finance Division, OMGUS, aufgelöst und durch Offices of the Economics bzw. Finance Adviser, OMGUS, ersetzt wurden, die - allerdings in vermindertem Umfang - die Aufgaben der bisherigen Fachabteilungen für Wirtschaft und Finanzen weiterhin wahrnahmen und an den bisherigen OMGUS-Instanzenzug gebunden blieben. Vorübergehende Erscheinungen blieben u. a. ein Cultural Affairs Adviser und ein Governmental Affairs Adviser to the Military Governor and the Deputy Military Governor.

Zur besseren Übersichtlichkeit sind die Inhaber auch der Positionen, die im folgenden nicht mehr eigens dargestellt werden, hier in Listenform zusammengestellt.

Ubersicht 30: Stellenbesetzung der Spitzenpositionen in der amerikanischen Militärregierung in Deutschland 1945-1949

Military Governor

Gen. Dwight D. Eisenhower $\quad$ bis 30.11 .1945

Gen. Joseph T. McNarney $\quad$ bis 15. 3.1947

Gen. Lucius D. Clay bis 15. 5.1949

John J. McCloy bis 21. 9.1949

Deputy Military Governor / Commanding General, USGCC, bzw. OMGUS

Gen. Lucius D. Clay

Maj.Gen. Frank A. Keating

bis 15.3 .1947

bis 30. 8.1947

Maj.Gen. George P. Hays

bis 21. 9.1949

Assistant (to) Deputy Military Govemor

Assistant Deputy for Resources Brig.Gen. William H. Draper

Juni - Oktober 1945

Assistant Deputy for Trade and Finance Laird Bell

Juni - Oktober 1945

Juni - Oktober 1945

Assistant Deputy for Public Services Maj.Gen. Oliver P. Echols

Juli 1945 - April 1946

Assistant Deputy Military Governor Maj.Gen. Oliver P. Echols

(seit 27. August 1945 Deputy Commanding General, USGCC)

Maj.Gen. Clarence L. Adcock

bis 15.10 .1946

${ }^{2}$ Das Wort „Adviser/Advisor" wird in den Quellen völlig willkürlich mit „e“ oder „o" geschrieben. Im „Handbuch“ wird die Schreibung Adviser verwendet. 
Maj.Gen. Frank A. Keating

Brig.Gen. Cornelius E. Ryan

Brig.Gen. William Hesketh

Chief Administrative Officer / Director of Administrative Services

Col. Nelson Dingley (Acting)

Col. James B. Edmunds

(seit 9.Oktober 1945 mit der neuen Amtsbezeichnung)

Col. John C. McCawley

Col. Bryan L. Milbum

Personnel Officer

Lt.Col. Ivan B. Baker

Col. Robert H. Chard

Lt.Col. James L. Williams

Col. Arthur H. Onthank

Lt.Col. Everett A. Luckenbach

Col. James T. Duke

Maj. Reginald J. Rivard (Acting)

Robert M. Bamett bis 15.3 .1947

bis 10. 9.1947

bis 1948

seit 20. 3.1945

seit 22. 4.1945

seit 27.6 .1945

seit $15.10 .1946^{3}$

seit 7.3 .1945

seit 17. 4.1945

seit 24. 9.1945

seit 28. 9.1945

seit 13. 5.1946

seit 25. 6.1946

seit 3.12 .1948

seit 4. 6.1949

\section{Chief of Staff}

Der Deputy Military Governor verfügte als Commanding General, USGCC bzw. OMGUS - wie andere Kommandobehörden auch - uber einen eigenen Stabschef. Dieser stand an der Spitze eines eigenen Office of the Chief of Staff, das in gewisser Hinsicht auch Leitungsfunktionen im Verhältnis zu anderen Staff Offices, insbesondere dem Secretary General, dem Personnel Officer, dem Director of Administrative Services sowie zunächst auch dem Adjutant General ausübte. Eine wesentliche Aufgabe bestand in der Koordinierung der Arbeit der Functional Offices und der Functional Divisions im Rahmen der Weisungen des Deputy Military Governor.

\section{Ubersicht 31: Stellenbesetzung des Chief of Staff, USGCC bzw. OMGUS,} 1945-1949

Brig.Gen. Bryan L. Milburn

Brig.Gen. Charles K. Gailey

Lt.Col. Milton L. Ogden
25.4.1945-15.10.1946

bis 10. 8.1949

bis 14. 8.1949

Im Zuge der Verlegung vielfältiger Organisationseinheiten von OMGUS in die amerikanische Zone wurde dort durch die beiden Allgemeinen Verfügungen Nr. 46 und 47 vom 1. November 1948 das Office of Deputy Chief of Staff, OMGUS, in Nürnberg eingerichtet und mit dem bisherigen Generalsekretär James E. King besetzt. Diese Dienststelle wurde am 20./21. März 1949 nach Bad Nauheim verlegt. Als das Office of the Chief of Staff am 14. August 1949 aufgelöst und seine Aufgaben in das Provisional Office of Administration, OMGUS, übergingen, erhielt der Deputy Chief of Staff in Bad Nauheim die Position eines Acting Assistant Director of the Provisional Office of Administration, OMGUS, während Milton L. Ogden als Berlin Representative in der alten Reichshauptstadt verblieb.

\footnotetext{
${ }^{3}$ Die Position wurde durch General Order Nr.71 vom 31.10.1946 abgeschafft.
} 


\section{Secretary General}

Die Position eines Secretary General ist erstmals in der General Order Nr.34 vom 29. Juni 1945 nachgewiesen. Vor diesem Zeitpunkt führte der Amtsinhaber die Bezeichnung Secretary. Er hatte allgemeine Koordinierungs- und Überwachungsfunktionen, die sich einer näheren Kompetenzbeschreibung entziehen. Dies wird nicht nur durch das Organigramm vom 7. Oktober 1945, sondern nicht zuletzt auch noch in dem am 12. Mai 1949 in den Military Government Regulations veröffentlichten General Statement of Functions deutlich. 4

Ubersicht 32: Personal von Secretary General, OMGUS, 1945 - 1949

Lt.Col. Charles E. Sands (Secretary)

Col. T. W. Hammond

Col. David Marcus

Col. William Whipple

Lt.Col. Charles F. Heasty (Acting)

Col. H. R. Maddux

James E. King

Eric G. Gration (Acting)

Lt.Col. Milton L. Ogden
7. 3. 1945 - 5. 5.1945

5. $5.1945-1.9 .1945$

1. $9.1945-30.4 .1946$

1. 5. $1946-15$. 3.1947

15. 3. $1947-19$. 5. 1947

19. 5. $1947-31.1 .1948$

31.1./19. 3. $1948-1.11 .1948$

1.11. 1948 - 7. 4.1949

7. 4. $1949-21.9 .1949$

Zeitweise war den Amtsinhabern ein Associate Secretary General beigegeben; vorübergehend gab es auch ein Office of the Staff Secretary.

\section{Adjutant General}

Der Adjutant General war vorbehaltlich der Zuständigkeit des Chief of Staff dafür verantwortlich, eine effiziente Verwaltung in der OMGUS-Zentrale zu sichem. Vor allem hatte er die Rechtmäßigkeit des Verwaltungshandelns der Militärregierung zu überwachen und war für Teilbereiche des Inneren Dienstes (Aktenführung, Vorbereitung von Befehlen und Anordnungen) verantwortlich. Zunächst dem Director of Administrative Services, USGCC, unterstellt, erlangte die Section bzw. das Office des Adjutant General im Frühjahr 1946 eine weitgehende organisatorische Eigenständigkeit. Während es zunächst nur grob in Untergliederungen für Cables, Top Secret Cables, Mail Distribution und Publications eingeteilt war, wurden erst Anfang 1947 Branches eingerichtet. Im Mai 1947 bestanden eine Message Control Branch, eine Miscellaneous Branch, eine Records Branch, eine Cable Control Branch, eine Publications Branch, eine Military Permit Branch, eine Travel Branch und die Reference Library, zu denen im Juli 1947 noch der Combined Travel Board trat. Diese Struktur änderte sich bis 1949 nicht entscheidend.

Ubersicht 33: Personal des Adjutant General, USGCC bzw. OMGUS 1945 - 1949

Lt.Col. James L. Williams

Col. Bruce Easley

Lt.Col. F. W. Marshall

Lt.Col. George H. Garde

Lt.Col. Frederick A. Sturm bis 4. 9.1945

bis 29.10. 1945

bis 25. 3. 1946

bis 6. 4.1949

bis 21. 9.1949

$4 / 370-2 / 18$. 


\section{Inspector General}

Durch General Order Nr. 70 vom 1. November 1946 wurde ein Office of Inspector General errichtet und Col. Harold Pinther zum Leiter bestellt. Sein Nachfolger wurde mit Wirkung vom 17. Dezember 1947 Col. Walter E. Jenkins, der offensichtlich bis zum Ende der OMGUS-Verwaltung im Amt blieb. ${ }^{5}$ Die Kompetenz ist inhaltlich mit .Inspektion nachgeordneter Kommandoeinheiten und Behörden“ korrekt bezeichnet.

\section{Control Office}

Durch General Order Nr. 53, USGCC, vom 13. September 1945 wurde als selbständige Section im Office of the Chief of Staff ein Control Office geschaffen. Es trat an die Stelle des früheren Reports Control Office. Col. William Whipple wurde zum ersten Control Officer der Militärregierung ernannt. Das neue Amt sollte die Durchfuhrung aller Fachprogramme der einzelnen Abteilungen von USGCC bzw. OMGUS ständig überwachen und zu diesem Zweck in erforderlichem Umfang Berichte anfordern. Ihm war weiter die Aufgabe gestellt, das Berichtsverfahren der Militärregierung insgesamt zu beobachten und die Informationen zu sammeln, welche für die Unterrichtung der zentralen Dienststellen in Washington erforderlich waren. Ferner hatte er die Kompetenz der Koordinierung des Personalwesens. SchlieBlich sollte das Control Office das Verwaltungsverfahren der Militärregierung ständig uberprufen und für Sonderaufgaben im Einzelfall zur Verfügung stehen. Vor Oktober 1945 gliederte sich das Amt in folgende Sections: Reorganization and Manpower Section unter Capt. F. T. Dunscomb, die Program Control Section unter Lt. D. G. White und die Reports Section unter dem Zivilbediensteten R. Elliott.

Im Oktober 1945 verfügte das Control Office über - zunächst zwei - Unterabteilungen: die Program Control Branch und die Reports Control Branch. Das Organisationshandbuch vom 9. März 1946 nennt eine Accounts Branch (Haushalts- und Rechnungswesen), eine Organization Branch, eine Audit Branch (Rechnungsprufung), eine Program Control Branch (Fachprogramme, Rechtsvorschriften, Spezialstudien), eine Reports Branch sowie schlieBlich eine Information and Statistics Branch. Inwieweit die Audit Branch mit dem durch General Order Nr. 4 vom 6. Februar 1946 innerhalb des Control Office geschaffenen Audit Office identisch war, lieB sich nicht genau feststellen. Vieles spricht dafür, daB der Unterschied im wesentlichen in der Bezeichnung des Leiters lag, der im Unterschied zu dem üblichen Titel Chief of Branch die besondere Bezeichnung Audit Officer führte.

Im weiteren Verlauf des Jahres 1946 änderten sich Zahl und Bezeichnungen der Unterabteilungen des Control Office mehrfach. So gab es Ende des Jahres die Budget Branch unter Ralph D. Snow, die Accounts Branch unter C. Hall, die Organization Branch unter W. H. Schroder, die Reports Branch unter John T. Butterwick, die Program Branch unter Milton E. Muelder und die Statistics Branch unter Burnham P. Beckwith. Control Officer war damals Col. David L. Robinson. Im Juni 1947 verfügte das Control Office unter dem damals amtierenden Control Officer, Waldemar Thorson, neben zwei Assistant Control Officers for Management bzw. for Statistical Standards über folgende

5 Jedenfalls hätte seine Abberufung in den General Orders 1949, von denen nur Nr. 38 nicht ermittelt werden konnte, bekanntgemacht werden müssen. 
sechs Unterabteilungen: Budget and Fiscal Branch unter Ralph D. Snow, Organization Branch unter W. H. Schroder, Graphics Branch unter E. E. Miner, Reports Branch unter Harold G. Carlson, Program Branch unter Milton E. Muelder und Statities Branch unter Burnham P. Beckwith; als Information Specialist fungierte Howard Denby. Unter dem neuen Control Officer, James L. Sundquist, der durch General Order Nr. 65 vom 14. August 1947 sein Amt erhielt, wurden die Organization Branch und die Program Branch unter Beryl R. McClaskey zur Organization and Program Branch zusammengefaBt. Gleichzeitig wurde der bisherige Assistant Control Officer for Management, Waldemar Thorson, zum Assistant Control Officer und damit zum ständigen Vertreter des Amtsleiters befördert. Durch General Order Nr.3 vom 26. Januar 1948 wurde unter Nutzung von Reichsmarkguthaben in der Verfügungsgewalt der Information Control Division ein OMGUS Reorientation Fund unter Verantwortung des Control Office geschaffen, um Projekte der Reorientation und Reeducation zu fördern.

Die fünf Unterabteilungen blieben bis Mitte 1948 bestehen, als das Control Office erneut umorganisiert wurde. Außer der weiter bestehenden Organization and Program Branch unter Gordon P. Freese und seiner Stellvertreterin, Beryl R. McClaskey, wurden drei vergleichsweise große Unterabteilungen geschaffen: die Reports and Statistics Branch unter H. R. Habicht, die Budget and Fiscal Branch unter Fletcher D. Mitchell sowie - etwas später - die Historical Branch unter David G. White. Außerdem wurde ein Assistant Control Officer for Bipartite Affairs, Albert G. Sims, ernannt, um James L. Sundquist in BICO-Angelegenheiten zu unterstützen.

Die wesentlichen Aufgaben der Historical Branch des Control Office bestanden in der Anforderung und Überprüfung historischer Berichte und anderer Darstellungen einzelner Verwaltungseinheiten von OMGUS einschließlich der Länderebene. Daraus sollte Material für eine umfassende Geschichte der Tätigkeit der amerikanischen Militärregierung in Deutschland geschöpft werden. Die Historiker, die innerhalb dieser Unterabteilung tätig waren, bereiteten auch Verwaltungsvorgänge aus einer ganzen Reihe von Fachabteilungen auf, um spätere historische Forschungen zu erleichtern. Eine abschließende Geschichte von OMGUS hat die Historical Branch nicht schreiben können, weil General Clay die ihm vorgelegten Entwürfe ablehnte und das Unternehmen im Frühjahr 1948 einstellen ließ. Die Unterabteilung wandte sich dann der Zusammenstellung von Material für eine Chronologie der Berliner Blockade der Jahre 1948/49 und der Bereitstellung von Materialien für die Memoiren Clays zu.

Für Personalangelegenheiten im engeren Sinne war das Control Office nicht zuständig; hierfür gab es eine eigene Verwaltungseinheit, die zuletzt die Bezeichnung Personnel Office führte.

Das Control Office stellte innerhalb von OMGUS sicherlich eine verwaltungsgeschichtliche Besonderheit dar. Als eine Mischung von Regierungszentrale und Organisationsreferat eines Fachmininisterium war die Dienststelle vor allen anderen Verwaltungseinheiten der Militärregierung herausgehoben. Inhaltlich ist noch einmal zu unterstreichen, daß das Control Office für die Vorbereitung der Bundesrepublik Deutschland von besonderer Bedeutung war. Seine wesentlichen Funktionen gingen im September 1949 auf das Office of Administration, HICOG, über.

\section{Office of the Director of Intelligence}

Vorläufer dieses Functional Office war die mit General Order Nr. 3 vom 25. November 1944 bei USGCC gegründete Intelligence Section, deren Chief auch Vorsitzender des Joint Intelligence Committee war, dem außer ihm auch die Leiter aller mit nachrich- 
tendienstlichen Funktionen betrauten Organisationseinheiten angehörten. Durch General Order Nr. 2 vom 24. Februar 1945 trat eine geringfügige formale Änderung ein, indem für den Ausschußvorsitzenden die Position eines Intelligence Officers eingerichtet wurde, so daß Col. Theodore J. Koenig in Personalunion Leiter der Intelligence Section war bzw. blieb. Das Office of the Director of Intelligence selbst wurde als ein Amt von USGCC am 25. April 1945 durch General Order Nr. 17 errichtet, um alle nachrichtendienstlichen Funktionen zu übernehmen, die bisher von entsprechend zuständigen Unterabteilungen innerhalb mehrerer Abteilungen von USGCC wahrgenommen worden waren. Zum Leiter wurde Brig.Gen. Thomas J. Betts bestellt, der am 30. August 1945 durch Brig.Gen. G. Bryan Conrad ersetzt wurde.

Die wesentlichen Funktionen von ODI blieben von der Umorganisation der amerikanischen Militärregierung im Oktober 1945 unberührt. In der Folgezeit bestanden von Liaison Officers abgesehen - drei Unterabteilungen, die Analysis (, Research) and Reports Branch, die Counter Intelligence Branch und die Civil Censorship Branch. Das Office of the Director of Intelligence war keine vorwiegend mit durchführender Tätigkeit betraute Verwaltungseinheit, sondern erhielt nur einen kleinen Mitarbeiterstab, der Rohmaterial nachrichtendienstlicher Relevanz auswertete und dabei auch Berichte anderer Abteilungen von OMGUS, nachrichtendienstlich tätiger Einheiten auf Landesebene und anderer Dienststellen, wie insbesondere G 2, USFET/EUCOM, berucksichtigte. Der Director of Intelligence vertrat die Vereinigten Staaten selbstverständlich auch im Quadripartite Intelligence Committee der alliierten Kontrollbehörde. Zu seinen Aufgaben gehörte schließlich auch die Dienstaufsicht über die durch General Order Nr.54 vom 23. Juni 1947 aufgelöste Field Information Agency, Technical, das Ministerial Collecting Center und das Berlin Document Center.

Am 25. Februar 1946 wurde das Joint Intelligence Committee durch das Intelligence Coordinating Committee ersetzt, in dem unter dem Vorsitz des Directors of Intelligence Vertreter folgender anderen Verwaltungseinheiten vertreten waren: Control Office, Office of Political Adviser, Information Control Service, Armed Forces Division, Economics Division und Public Safety Branch der Internal Affairs and Communications Division. Am 17. Juli 1946 wurde Col. Theodore J. Koenig für die Zeit bis zum 3. November dieses Jahres erneut zum Direktor ernannt, dann jedoch von Col. Peter P. Rodes abgelöst.

1946/47 wurde die Analysis Research and Reports Branch von Lawrence E. DeNeufville, die Counterintelligence Branch von Maj. $\mathrm{H}$. Lindjord und die Civil Censorship Branch von E. Kabzenell geleitet. Im Jahre 1948 gliederte sich das Amt - von einer Administration (and Personnel) Branch abgesehen - nur noch in zwei Unterabteilungen: die Analysis and Research Branch unter Lawrence E. DeNeufville und die Security Branch unter Laughlin A. Campbell. Zu den mehrfach genannten beiden Unterabteilungen - die Analysis and Research Branch erhielt mit Innis D. Harris, die Security Branch mit C. J. O'Connor jeweils neue Leiter - traten Anfang 1949 eine Plans and Policy Branch unter Lt.Col. J. J. Eason, eine Liaison Branch unter J. S. Arouet und eine Special Project Branch unter Hans Kallman. Außer dem Direktor selbst und Kallman hatten alle anderen Leiter ihren Dienstsitz damals in Bad Nauheim. Das Office blieb im September 1949 als Office of Intelligence, HICOG, im ganzen in seinen Kompetenzen erhalten. 


\section{US Political Adviser / Office of the Director of Political Affairs}

Die Funktion des Political Adviser, die Ambassador Robert D. Murphy fast während der gesamten OMGUS-Periode, sein zunächst geschäftsführender Nachfolger James W. Riddleberger seit Fruhjahr 1949 ausubten, bestand ausschlieBlich darin, die Interessen des amerikanischen Außenministeriums in Deutschland zu vertreten und dieses Ministerium über Politik und Tătigkeit der Militärregierung zu unterrichten. Die Aufgaben des Office of the Director of Political Affairs, das im Unterschied zum Political Adviser voll in den Verwaltungsaufbau der Militärregierung integriert war, lagen dagegen darin, unmittelbar Verantwortung fur alle „politischen“ Aktivitäten in Deutschland zu übernehmen.

Die Keimzelle des Office of the Director of Political Affairs lag in der Military Government Division B, USGCC. General Order Nr. 3 vom 25. November 1944 führte eine Political Division unter Leitung von Robert D. Murphy auf, die sich im Fruhjahr 1945 in eine Political Affairs Branch, eine Political Reporting and Intelligence Branch, eine Protection of American Interests Branch, eine Economics Branch, eine Information Services Branch, eine Displaced Persons Branch, eine Administrative Branch und eine Russian Liaison Branch gliederte. Durch General Order Nr. 33 vom 29. Juni 1945 wurde innerhalb des damals geschaffenen Executive Office die Position eines Political Adviser eingerichtet und mit Murphy besetzt. Unabhängig davon schuf man das Amt eines Director of Political Affairs, das Donald R. Heath ubertragen wurde. Die Aufgabenverteilung zwischen Murphy und Heath wurde durch die Umwandlung von USGCC in OMGUS nicht berührt.

Das Office of the Director of Political Affairs - diese Bezeichnung taucht erstmals im Oktober 1945 auf - bestand damals aus der Denazification Branch und der Political Branch, beide unter J. J. Muccio, sowie der Intelligence Branch unter Col. W. D. Hohenthal, der Economics Branch unter Loyd V. Steere und der American Interests Branch unter A. D. Hodgdon.

Die Bezeichnung Office of the Director of Political Affairs (OPA) wurde dann im Organisationshandbuch vom März 1946 ausdrücklich bestätigt. Das Amt gehörte zu den Functional Offices, für die ausführliche Funktionsbeschreibungen vorliegen.

Zur Erläuterung des unbestimmten Begriffes „political“ mag die Beschreibung der Aufgaben des Direktors vollständig wiedergegeben werden; sie gehen weit über den engeren Bereich des Diplomatischen hinaus:

1. Politische Aspekte der Grundsätze der Entnazifizierung.

2. Grundsätze und Programme für die Bildung und Überwachung der deutschen politischen Parteien.

3. Einsatz und Verteilung der ehemaligen Angehörigen des deutschen Auswärtigen Amtes.

4. Beteiligung an der Überwachung und Gestaltung der Beziehungen des Kontrollrates zu Drittstaaten.

5. Beteiligung im Rahmen des Editorial Board an der Vorbereitung des Monatsberichts des Militärgouverneurs.

6. Beratung in „politischen“ Fragen auf Wunsch anderer Ämter, Abteilungen oder nachgeordneter Stellen der Militärregierung.

7. Fühlungnahme mit dem Department of State, soweit dazu ermächtigt.

8. Unterrichtung des Political Adviser über die Entwicklung der öffentlichen Meinung in den Vereinigten Staaten und im Ausland. 
9. Politische Überprüfung von Ernennungen in den deutschen zentralen Behörden oder von Beratern der Militärregierung.

10. Beteiligung bei der Wiedererrichtung deutscher zentraler Verwaltungsbehörden.

11. Beteiligung an Untersuchungen über wirtschaftliche Zusammenschlüsse (Kartelle) unter politischen Gesichtspunkten.

12. Unterrichtung des Political Adviser über die gegenwärtige Lage in Deutschland.

13. Versorgung anderer Abteilung von OMGUS mit Berichten und sonstigen Informationen amerikanischer diplomatischer Missionen und aus anderen Quellen.

14. Auswertung der Akten des deutschen Auswärtigen Amtes und der Reichskanzlei sowie der Kenntnisse des entsprechenden deutschen Personals.

15. Vertretung amerikanischer Interessen im PaBwesen, bei der Feststellung der Identität von Personen einschlieBlich der Ausgabe von Einreisesichtvermerken.

16. Verbindungsstelle der Vereinigten Staaten zwischen der Alliierten Kontrollbehörde und Drittstaaten ohne Anteil an der Militärregierung in Deutschland.

17. Bereitstellung von Political Officers für die Leiter der drei Landesmilitärregierungen in der amerikanischen Zone und für die Sitzungen des Länderrats in Stuttgart.

Das aus dem State Department stammende Personal, also vor allem Murphy und seine Mitarbeiter, aber auch die Konsulatsbeamten, blieben formal Angehörige des Geschäftsbereichs des Außenministeriums, wurden aber von dem voll in die Militärregierung integrierten und damit zum Geschäftsbereich des War Department gehörenden Office of the Director of Political Affairs in allen Angelegenheiten der Dienststellenverwaltung betreut.

Nachdem Clay am 15. März 1947 die Funktionen eines Commander-in-Chief, European Command, and Military Governor ubernommen hatte, gehörte der Political Adviser Murphy nicht einmal mehr im Sinne einer wie immer gearteten losen Anbindung zu OMGUS, sondem war mit seinen Mitarbeitern Teil des Office of the Commander-in-Chief, European Command, und fuhrte die Bezeichnung US Political Adviser. Er beriet Clay sowohl für den militärischen Bereich des Headquarters European Command einschlieBlich dessen nachgeordneter militărischer Stellen und Einheiten als auch für den Bereich des Office of Military Government in dem oben geschilderten weiten Sinne des Begriffs „politisch“.

Das Office of the Director of Political Affairs, OMGUS, das in der ersten Jahreshälfte 1947 weiterhin von Donald R. Heath, ab 11. August 1947 geschäftsführend von dessen bisherigem Stellvertreter, Loyd V. Steere, und ab 30. Oktober 1947 von James W. Riddleberger geleitet wurde, gliederte sich - von der Administrative Branch abgesehen - in Unterabteilungen für Information Control (später Press and Public Relations) unter Thomas J. B. Wenner, Economics unter Loyd V. Steere (später Wesley C. Haraldson), Exploitation German Archives unter W. W. Blancke und Political Branch unter Warren M. Chase.

Die Zahl der Unterabteilungen blieb 1948 konstant, doch trat insoweit eine wesentliche Änderung ein, als an die Stelle der Exploitation German Archives Branch eine Consular Branch unter Marshall M. Vance trat, welche die Aufsicht über die sieben amerikanischen Konsulate (Consular Offices) in Berlin, Bremen, Bremerhaven, Frankfurt am Main, Hamburg, München und Stuttgart führte.

Hervorzuhebendes Merkmal für die Entwicklung auch dieses Office im Jahre 1949 ist eine Teilverlegung von Berlin in die amerikanische Zone, in diesem Fall nach Frankfurt am Main. In Berlin blieben neben dem Leiter Riddleberger zwar die seit 1948 von James W. Gantenbein geleitete Economics Branch und die Political Branch - nun- 
mehr unter Perry Laukhuff; sie verfügten aber beide über Außenstellen in Frankfurt, das auch Sitz eines Chief, POLAD/Frankfurt (William Bruce Lockling) war. In Frankfurt wurde außerdem eine Foreign Representation Branch unter William Schott errichtet.

Die wesentlichen Funktionen des Office of the Director of Political Affairs wurden beim Übergang von OMGUS zu HICOG in einem Office of Political Affairs, HICOG, unter Leitung von James W. Riddleberger zusammengefaßt.

\section{Office of the Director of Public Information}

Durch General Order Nr. 8 vom 29. März 1945 wurde neben dem Information Control Service ein Public Relations Services bei USGCC errichtet und dem Commanding General, USGCC, unmittelbar unterstellt. Leiter dieses Dienstes, der für Öffentlichkeitsarbeit zuständig war, wurde gleichzeitig Brig.Gen. Frank A. Allen, der mit Wirkung vom 15. Oktober 1945 durch Oberst Frances F. Fitzgerald, dieser ab 26. November 1945 durch Oberst Clarence E. Lovejoy als Public Relations Officer ersetzt wurde. Am 5. April 1946 wurde Brig.Gen. Charles K. Gailey zum Director of Public Relations ernannt, an dessen Stelle mit Wirkung vom 16. Dezember 1946 geschäftsführend William Haggard trat. Am 10.Juni 1946 wurde die Bezeichnung Public Relations Office bzw. Director of Public Relations durch Public Information Office bzw. Director of Public Information ersetzt.

Durch General Order Nr. 48 vom 2. November 1948 wurde unter dem Director of Public Information, OMGUS, ein Public Information Office, OMGUS (Frankfurt), errichtet, dem man auch die bisherigen Aufgaben des Bipartite Control Office und der Joint Export-Import Agency auf dem Gebiet der Öffentlichkeitsarbeit übertrug. Der Schwerpunkt der Tätigkeit dieser neuen Frankfurter Stelle lag ganz eindeutig in der Unterrichtung der deutschen Öffentlichkeit über die Wirtschaftspolitik der Vereinigten Staaten, nicht zuletzt über das European Recovery Program.

Zur Vorbereitung des Übergang von OMGUS auf HICOG wurde durch General Order Nr. 44 vom 3. September 1949 ein Provisional Office of Public Affairs, OMGUS, geschaffen und Ralph Nicholson zum geschäftsführenden Director of Public Affairs ernannt. Bei dieser Gelegenheit wurden auch die Funktionen der Information Services Division und der Education and Cultural Relations Division zusammen mit denen des Public Information Office auf das neue Amt übertragen.

\section{Economics Division / Office of the Economics Adviser}

Ausgangspunkt für eine Betrachtung der Entwicklung der Wirtschaftsabteilung der amerikanischen Militärregierung in Deutschland ist General Order Nr.90, ETO, vom 9. August 1944, durch die Fragen der Wirtschaftsplanung innerhalb der damals gegründeten USGCC der Military Government Division A unter General Eric F. Wood zugewiesen wurden. Durch General Order Nr.3, USGCC, vom 25. November 1944 wurde im Rahmen der Neuorganisation von USGCC eine eigene Economics Division unter Col. Graham K. Howard als geschäftsführendem Leiter errichtet. Als eine der damals geschaffenen zwölf Abteilungen gehörte die Economics Division zu den klassischen Verwaltungseinheiten der Militärregierung. Der Leiter dieser Wirtschaftsabteilung war von Anfang an auch dadurch besonders ausgezeichnet worden, daß er dem Joint Intelligence Committee angehörte, ein Umstand, der verdeutlicht, daß die Auf- 
gaben der Abteilung damals nahezu ausschließlich in der Planung und Informationsbeschaffung lagen.

Am 24. März 1945 wurde der amtierende Leiter der Economics Division, Graham K. Howard, durch Col. John R. Gilchrist abgelöst, der seinerseits am 5. Mai 1945 durch Brig.Gen. William H. Draper ersetzt, allerdings dessen Stellvertreter blieb. Draper hat sein Amt auf ausdrücklichen Wunsch Clays übernommen und gehörte nicht nur wegen seiner Stellung zu den bedeutendsten Köpfen der Militärregierung.

Außerdem gab es damals eine Reparations, Deliveries and Restitution Division unter dem amtierenden Direktor Col. C. S. Reid, der jedoch schon Anfang Februar durch Col. H. C. Newton bzw. am 23. Mai 1945 durch Col. Leslie W. Jefferson abgelöst wurde. Nachdem Deutschland vollständig von den Alliierten erobert worden war, kam die Planungsphase wie selbstverständlich zum AbschluB, so daß USGCC auch im Bereich der Wirtschaftsverwaltung völlig reorganisiert wurde. Durch General Order Nr.33 vom 29. Juni 1945 blieb die Reparations, Deliveries and Restitution Division zwar bestehen, die Economics Division wurde aber in eine Industry Division und eine Food and Agriculture Division aufgeteilt. Die Zuständigkeiten der erstgenannten Abteilung erstreckten sich nicht nur auf den in ihren Namen genannten Bereich, sondern auch auf die Vermögensüberwachung und den Schutz von Denkmälern, Kunstwerken und Archiven. Der Komplex Industry wurde im Geschäftsverteilungsplan durch die Stichworte Brennstoffe, Bergbau, Metalle, Chemikalien, Industrieausrustung und Maschinen, Konsumgüter, Baumaterialien und öffentliche Einrichtungen gekennzeichnet. Die Abteilung für Ernährung und Landwirtschaft umfaßte auch das Forstwesen.

Alle drei Abteilungen erhielten die allgemeinen Richtlinien für ihre Arbeit von dem oder über den Assistant Deputy for Resources. Diese Position erhielt der bisherige und spätere Direktor der Wirtschaftsabteilung, Brig.Gen. William H. Draper, der auch mit der Wahrnehmung der amerikanischen Interessen im zuständigen Directorate der Alliierten Kontrollbehörde beauftragt wurde. Die Reparations, Deliveries and Restitution Division wurde auch weiterhin von Col. Leslie W. Jefferson geleitet, während Col. James Boyd, der vorherige Leiter der Industry Branch in der Economics Division, mit der Leitung der Industrieabteilung betraut und der Zivilbedienstete Loyd V. Steere mit der Wahrnehmung der Geschäfte des Direktors der Abteilung für Ernährung und Landwirtschaft beauftragt wurde.

Zum Bereich der Wirtschaftsverwaltung im weiteren Sinne gehörte sicherlich auch die kurzlebige Division of Trade and Commerce unter Frederick Winant, die indes zusammen mit der Finanzabteilung der Aufsicht des Assistant Deputy for Trade and Finance, Laird Bell, unterstellt war.

Eine andere Traditionslinie der Economics Division führt zurück in den April 1945, als eine Production Control Agency unter der Aufsicht der Abteilung G-4, SHAEF, geschaffen wurde. Im Mai trat eine Economics Control Agency unter G-5, SHAEF, hinzu. Beide Stellen wurden im Juni in Branches ihrer jeweiligen Abteilung umgewandelt. Die Unterabteilung für Wirtschaft bei G-5, SHAEF, hatte fünf Sections: Trade and Commerce, Food and Agriculture, Road Transport, Manpower and Removal, Destruction and Reparations. Nach der Auflösung von SHAEF am 14. Juli 1945 wurde die Unterabteilung für Produktionsüberwachung der Aufsicht von G-5, USFET, an Stelle von G-4, USFET, unterstellt. Gleichzeitig mit diesen Änderungen waren die vier oben genannten Abteilungen bei USGCC gebildet worden. Die Leiter der entsprechenden Abteilungen bei USGCC waren gleichzeitig Leiter der Unterabteilungen von USFET.

Nach Errichtung von OMGUS wurde durch General Order Nr.60, OMGUS, vom 2. Oktober 1945 neben entsprechenden Ernennungen eines Legal und eines Financial 
Adviser auch ein Economics Adviser to the Office of Military Government for Germany (US) bekanntgegeben; die Position erhielt Brig.Gen. William H. Draper, der durch General Order Nr.63, OMGUS, vom 9. Oktober 1945 mit Wirkung vom 15. Oktober Leiter auch einer neuen Economics Division wurde. Sitz der Abteilung war Berlin mit einer AuBenstelle in Frankfurt, die verwaltungsmäBig ein Teil von USFET war, fachlich jedoch den Weisungen aus Berlin unterworfen war. So gab es dann für einige Monate eine Wirtschaftsabteilung Economics Division, OMGUS und eine Wirtschaftabteilung Economics Division, OMGUSZ, wobei letztere auch unter der Behördenfirma Economics Division, USFET OMGUSZ, firmierte.

Die General Order Nr.63, OMGUS, vom 9. Oktober 1945 enthielt nicht nur Personalentscheidungen, sondern verdeutlichte auch, daß mit Wirkung vom 15. Oktober 1945 die folgenden bisherigen Abteilungen, Stabsämter und Unterabteilungen zur Economics Division zusammengefaßt waren: Office of Assistant Deputy for Resources, Industry Division, Food and Agriculture Divison, Trade and Commerce Division sowie die Unterabteilungen Reparations and Restitution und Monuments, Fine Arts and Archives der damals aufgelösten Reparations, Deliveries and Restitution Division.

Damit ergab sich folgende Organisationsstruktur der Economics Division: Neben dem Office des Direktors mit insgesamt fünf Bediensteten und einem Executive Office (Dienstellenverwaltung einschließlich eines Zentralsekretariats) mit mindestens sechs Bediensteten gab es fünf Unterabteilungen. Von ihnen kümmerte sich - wie der Name sagt - die Requirements and Allocations Branch unter 1 st Lt. M. Hoobs ebenfalls weitgehend um Probleme der Dienststellenverwaltung, während sich die Policy and Control Branch unter S. Wennberg mit Grundsatzfragen und der Überwachung deutscher Dienststellen zu beschäftigen hatte. Als Fachunterabteilungen im engeren Sinne sind dagegen die Food and Agriculture Branch, die Restitution Branch und die Industry Branch anzusehen. In diesen drei Unterabteilungen verrichteten etwa $90 \%$ der mehr als 150 Bediensteten ihre Arbeit.

Die Unterabteilung für Ernährung und Landwirtschaft unter Brig.Gen. Hugh B. Hester gliederte sich in acht Referate: Land, Distribution, Institutions, Production, Reports and Statistics, Rationing, Transportation, Storage and Commodities Section. Während die Restitution Branch nicht systematisch untergliedert war, umfaßte die Industrieabteilung folgende Referate:

Ubersicht 34: Organisation der Industry Branch, Economics Division, OMGUS, Oktober 1945

- Chemical Section

- Plastics and Protective Coatings Section

- Vaccines, Sera and Antitoxins Section

- Building Materials and Construction Section

- Coal and Non-metallic Mining Section

- Intelligence Section

- Legal Section

- Machinery and Optics Section

- Public Utilities Section

- Oil Section

- Metals Section

Hinzu kamen noch einige Sonderbeauftragte. Personell hatte sich insoweit nichts geändert, als weiterhin Col. Leslie W. Jefferson an der Spitze der Restitutions Branch stand und Col. James Boyd die Industry Branch leitete. 
In den Zusammenhang dieser Darstellung gehört der Sache nach auch die durch General Order Nr. 42 vom 12. September 1945 gegründete Investigation of Cartels and External Assets Division unter Col. Bernard Bernstein. Sie umfaßte neben dem Office of the Director sieben Unterabteilungen und einen Planungsstab. Durch General Order Nr.73, OMGUS, vom 14. Dezember 1945 wurde die Abteilung jedoch bereits wieder aufgelöst und ihre Zuständigkeit auf die Wirtschafts- und Finanzabteilung übertragen. Dabei übernahm die Wirtschaftsabteilung die Verantwortung für Erhebungen uber Art und Zielsetzung bestehender deutscher Kartelle, Syndikate, Trusts und anderer Formen wirtschaftlicher Konzentration. Diese Zuständigkeiten wurden in der Decartelization Branch unter Philip Hawkins zusammengefaBt. In der Finance Division wurde eine External Assets Branch unter Theodore H. Ball errichtet. Am 14. Dezember 1945 erloschen ebenfalls die Funktionen des I. G. Farben Control Officer, dessen Zuständigkeiten ebenfalls zum gröBeren Teil von der Wirtschaftsabteilung übernommen wurden.

Am 15. Januar 1946 bestand die Wirtschaftsabteilung außer dem in Referate eingeteilten Stab aus folgenden Unterabteilungen:

Ubersicht 35: Organisation der Economics Division, OMGUS, 15. Januar 1946

- Policy and Coordination Branch

- Reports and Statistics Branch

- Decartelization Branch

- Food and Agriculture Branch

- Trade and Commerce Branch

- Restitution Branch

- Industry Branch

Hinzu trat durch General Order Nr. 10, OMGUS, vom 8. Mărz 1946 eine Supply Accounting Branch, die vor allem für Vorratsbewirtschaftung verantwortlich war. Das Organisationshandbuch vom 9.März 1946 zeigt einen auf sechs Referate erweiterten Stab sowie sechs Unterabteilungen:

Ubersicht 36: Organisation der Economics Division, OMGUS, 9. März 1946

- Industry Branch

- Food and Agriculture Branch

- Trade and Commerce Branch

- Decartelization Branch

- Restitutions Branch

- Supply Accounting Branch

Dazu kamen noch einige nachgeordnete Stellen vor allem im Bereich der wirtschaftlichen Entflechtung, insbesondere der I. G. Farben AG. Im Laufe des Jahres 1946 ergab sich insoweit eine bedeutende Änderung für die amerikanische Wirtschaftsverwaltung in Deutschland, als erste Schritte zur Errichtung der Bizone eingeleitet wurden. Diese führten jedoch nicht zu organisatorischen Konsequenzen, zumal die Ernennung des Economics Adviser und Leiters der Wirtschaftsabteilung, Brig.Gen. William H. Draper, durch General Order Nr. 14, OMGUS, vom 28. Februar 1947 zum Economics Adviser to the Deputy Military Governor eine mit Sicherheit unpolitische Entscheidung war. Dessen Nachfolger als Leiter der Wirtschaftsabteilung wurde Menc S. Szymczak, der seinerseits jedoch schon am 26. Juni 1947 durch einen anderen Zivilbediensteten, Lawrence Wilkinson, ersetzt wurde. 
Im März 1947 umfaßte die Abteilung - außer den bereits genannten - noch zwei weitere Unterabteilungen. Die Notwendigkeit einer Research Control Branch unter George Scatchard wurde in einem Bericht, der im Bulletin der Militärregierung erschien, mit der lakonischen Feststellung begründet, daB moderne Kriege unter Umständen in einem Teströhrchen beginnen könnten. ${ }^{6}$ Die zweite neue Unterabteilung war die Requirements and Allocations Branch unter George N. McClusky.

Übersicht 37: Organisation und Personal der Economics Division, OMGUS, Juli 1947

- Administration and Personnel Branch (Lt.Col. Thurman S. Doman)

- Policy and Coordination Branch (Malcolm S. McComb)

- Reports and Statistics Branch (Saul Nelson)

- Requirements and Allocation Branch (George N. McClusky)

- Research Control Branch (George Scatchard, später C. H. Nordstrom)

- Decartelization Branch (James S. Martin)

- Food and Agriculture Branch (Col. Hugh B. Hester)

- Trade and Commerce Branch (Spencer Phenix)

- Restitution Branch (Col. John H. Allen)

- Industry Branch (H. L. Berno)

- Supply Accounting Branch (Moore C. McIntosh, später John Kovac)

Die ersten fünf genannten Unterabteilungen sowie das amerikanische Sekretariat des Economics Directorate bei der Alliierten Kontrollbehörde wurden damals als Staff Branches, die übrigen Unterabteilungen als Functional Branches bezeichnet. Das einschlägige Organigramm enthält schlieBlich die amerikanischen Anteile (US Elements) in den Bipartite Control Groups (Economics, Food and Agriculture) und bietet insoweit eine nahezu vollständige Übersicht über die Wirtschaftsverwaltung der amerikanischen Militärregierung in Deutschland. Die große politische Bedeutung der Economics Division im Zusammenhang mit der Errichtung der Bizone kam organisatorisch besonders dadurch zum Ausdruck, daß die Direktoren Draper bzw. Wilkinson drei Stellvertreter erhielten, die alle nicht an der Spitze einer Unterabteilung standen: einen Deputy Director for Negotiations (Don D. Humphrey), einen Deputy Director for Administration (Col. Stephen A. Park) und einen Deputy Director for Bizonal/Minden Operations (Peter V. Martin, später A. S. Barrows).

Durch General Order Nr. 18, EUCOM, wurde die Wirtschaftsabteilung aufgelöst und mit Wirkung vom 1.März 1948 das Office of the Economics Adviser, OMGUS, geschaffen; die meisten Fachaufgaben im Bereich der Wirtschaftsverwaltung waren inzwischen von unterschiedlichen Dienststellen in der Bizone übernommen worden. Der Economics Adviser, der bisherige Direktor der Wirtschaftsabteilung, Lawrence Wilkinson, und sein vergleichsweise kleiner Mitarbeiterstab blieben für die Entwicklung der Grundsätze der Wirtschaftspolitik verantwortlich, die vom amerikanischen Anteil im Bipartite Control Office vertreten wurde. Durch die Reorganisation im $\mathrm{Be}$ reich der Innen- und Kulturverwaltung, die aufgrund der General Order Nr.6 vom 18. Februar 1948 durchgeführt wurde, war die Wirtschaftsverwaltung insoweit betroffen, als die Aufgaben der Communications Branch der damals aufgelösten Internal Affairs and Communications Division mit Wirkung vom 1.März $1948 \mathrm{dem}$ Office of the Economics Adviser übertragen wurden. Damals löste man auch die Transport

6 "Modern wars can start in a test tube“ (Military Government Weekly Information Bulletin Nr.82 vom 3.3.1947). 
Division auf und übertrug deren Aufgaben, soweit sie nicht auf BICO oder deutsche bizonale Stellen übergingen, dem Office of the Economics Adviser.

Im März 1948 gliederte sich das Office des Economics Adviser in zwei Stabsreferate (Verwaltung, Zentralsekretariat) sowie sechs Fachgruppen (Groups): Industry unter Frank L. Mayer, Communications unter Eugene H. Merrill, Food and Agriculture unter R. A. Ionnes, Transport unter dem bisherigen Leiter der Verkehrsabteilung, Col. Hans W. Holmer, Reports and Statistics unter Saul Nelson und Research Control unter C. H. Nordstrom. Diese Verwaltungsstruktur blieb bis in das Jahr 1949 bestehen, sieht man von kleineren Veränderungen der Bezeichnung einzelner Gruppen einmal ab; so nennt eine Personalübersicht vom April 1949 die bisherige Research Control Group nunmehr Scientific Research Group. Personell hatte sich insoweit etwas geändert. als nur die Fachgruppen für Industrie und für Post- und Fernmeldewesen (Communications) ihre Leiter behalten hatten. An der Spitze der Food and Agriculture Group stand nunmehr Philip M. Raup, während die Transport Group von W. T. Cantrell und die Reports and Statistics Group von C. K. Nichols geleitet wurden. Am inhaltlichen Auftrag des Office änderte sich dadurch kaum etwas. Indes gab es keine Decartelization Group mehr, da die Zuständigkeit für die Entflechtung der Wirtschaft vollkommen auf BICO übergegangen war, nur für eine sehr kurze Zeit gab es eine Decartelization Branch in der Property Division. Von räumlichen Verlagerungen war das Office of the Econommics Adviser nicht betroffen; es blieb vollständig in Berlin.

Mit General Order Nr.46 vom 3.September 1949 gingen die Zuständigkeiten des Office of the Economics Adviser und des US Chairman of BICO zusammen mit denen des Office of the Finance Adviser und der Finance Division auf das in Frankfurt am Main errichtete Provisional Office of Economic Affairs, OMGUS, über. Als am 21.September 1949 der Hohe Kommissar endgültig an die Stelle des Militärgouverneurs trat, wandelte sich dieses Amt endgultig zum Office of Economic Affairs, HICOG, wobei der Problemkreis der Entflechtung der Wirtschaft dem Office of the General Counsel zugeschlagen wurde.

Am Beispiel der Wirtschaftsverwaltung von OMGUS - der Economics Division, des Office of the Economics Adviser und des Bipartite Control Office - kann am anschaulichsten gezeigt werden, in welchen Stufen sich die amerikanische Besatzungsmacht von der unmittelbaren Überwachung deutscher Verwaltungstätigkeit zurückzog und ihre vergleichbaren Fachbehörden entsprechend umorganisierte. Insofern ist die Geschichte der Wirtschaftsabteilung von OMGUS nicht nur von fachlicher, sondern von allgemein historischer Bedeutung.

\section{Finance Division / Office of the Finance Adviser}

Die Ursprünge des Office of the Finance Adviser, OMGUS, und seines Vorgängers, der Finance Division, OMGUS, können bis zum 14. August 1944 zurückverfolgt werden, als die Civil Affairs Division, SHAEF, eine German Country Section mit einer Finanzabteilung errichtete. Zuständigkeiten auf dem Bereich der Finanzverwaltung zum Zeitpunkt der Errichtung von USGCC am 9. August 1944, insbesondere die Formulierung von Grundsätzen einer künftigen Finanzpolitik der Besatzungsverwaltung, die Vorbereitung des Abschnitts „Finanzen“ im Handbuch der Militärregierung und die Sammlung von Nachrichten zur deutschen Finanzverfassung lagen dann im Zuständigkeitsbereich der Military Government Division A, USGCC. Am 25. November 1944 wurde mit der Einführung der Abteilungsstruktur bei USGCC eine eigene Finanzabteilung unter Col. Bernard Bernstein errichtet. 
Bei der Organisationsreform vom 29. Juni 1945 wurde die Finance Division dem Assistant Deputy for Trade and Finance unterstellt und umfaBte Branches für Public Finance unter Lt.Col. M. P. Fisher, für Financial Institutions unter Lt.Col. J. E. McDonougy, für Foreign Exchange and Blocking Control unter O. A. Schmitt, für Intelligence and Liaison unter A. M. Kamarck, für Accounts and Audits unter Col. M. J. Hudtluff sowie für Currency unter Lt.Col. H. D. Cragon.

Als USGCC mit Wirkung vom 1. bzw. 15. Oktober 1945 als OMGUS organisiert wurde, entstand die Finance Division, OMGUS, die für die allgemeine Aufsicht und Kontrolle über die öffentlichen Finanzen in der amerikanischen Besatzungszone, Fragen der Devisenbewirtschaft und des deutschen Auslandsvermögens zuständig war. Außerdem hatte sie die Sicherung entsprechender Devisen aus Exportleistungen und für die Bezahlung von Importen vorzunehmen. Auch der Bereich Geld und Kredit einschlieBlich der Aufsicht über die Landeszentralbanken lag bei der Finance Division. Leiter dieser Abteilung wurde der bisherige Assistant Deputy for Trade and Finance, Joseph M. Dodge, unter nahezu gleichzeitiger Ernennung zum Financial Adviser.

Als Stellvertreter des Abteilungsleiters fungierte Col. David L. Robinson. AuBer dem Office of the Assistant Deputy for Trade and Finance waren auch die Property Control Branch der bisherigen Reparations, Deliveries and Restitution Division und Teile des Office of the Control Officer, I. G. Farbenindustrie A. G., in der Finance Division aufgegangen. Mitte Dezember 1945 kam die Kompetenz für die Ermittlung deutscher Auslandsvermögen hinzu, soweit diese innerhalb Deutschlands vorzunehmen war.

Die Leitung der fortbestehenden Financial Institutions Branch lag im Oktober 1945 bei Walter Lichtenstein, der Foreign Exchange and Blocking Control Branch bei Jack Bennett, der Financial Intelligence and Liaison Branch bei A. M. Kamarck, der Currency Branch weiterhin bei Lt.Col. H. D. Cragon, der Accounts and Audit Branch weiterhin bei Col. M. S. Hudtluff, der Property Control Branch bei Col. C. S. Reid sowie der I.G. Farben Control Branch bei Col. E. S. Pillsbury.

Das Organisationshandbuch vom 15. März 1946 wies nur eine leicht veränderte Organisationsstruktur der Abteilung auf. Vom 9. Juni 1946 bis 4. März 1947 wurde die Abteilung von Jack Bennett geleitet. An der Spitze der Financial Institutions Branch stand weiterhin Walter Lichtenstein; die Public Finance Branch wurde von Robert P. March, die Property Control Branch von E. N. Reinsel, die External Assets and Intelligence Branch von Albert Bender, die Foreign Exchange and Blocking Control Branch von Jo Fisher Freeman sowie schließlich das Foreign Exchange Depository in Frankfurt von Col. William G. Brey geleitet. Seit dem 4. März 1947 finden wir Theodore H. Ball als Abteilungsleiter, Jo Fisher Freeman als dessen Stellvertreter und folgende neue Unterabteilungsleiter: Howard P. Jones (Public Finance), Samuel M. Rose (External Assets and Intelligence), Judson C. Jones (Foreign Exchange and Blocking Control) sowie Richard P. Aikin (Finance Institutions); kurzfristig bestand eine Reports and Statistics Branch unter David Schwartz.

Die Abteilung war also bis Februar 1948 mehrfach innerorganisatorischen Änderungen unterworfen, die auch die Bezeichnungen und Funktionen der Unterabteilungen betrafen. Als die amerikanische und britische Besatzungsverwaltung sowie die deutschen bizonalen Dienststellen in den Jahren 1947 und 1948 reorganisiert wurden, übertrug man in größerem Umfang Durchführungskompetenzen auch auf dem Gebiet der Finanzverwaltung auf die deutschen Behörden. Das Ende dieser Entwicklung markierte in Bezug auf die amerikanische Militärregierung General Order Nr. 18, EUCOM, vom 18. Februar 1948, durch welche die Finanzabteilung der Militärregierung ebenso aufgelöst wurde wie das Office of the Finance Adviser, CINCEUR. An deren Stelle trat das Office of the Finance Adviser, OMGUS, und eine Property Division, 
OMGUS. Die Aufsicht über die Durchführung der allgemeinen Finanzpolitik im Vereinigten Wirtschaftsgebiet war der neu geschaffenen Bipartite Finance Group innerhalb des Bipartite Control Office übertragen worden.

Das Office of the Finance Adviser unter Jack Bennett sowie dessen Stellvertreter und Nachfolger Jo Fisher Freeman (geschäftsführend ab 18. Januar 1949) übernahm von der früheren Finanzabteilung die Zuständigkeiten der Überprüfung der deutschen Finanzpolitik sowie der Beschäftigung mit mulitilateralen Übereinkommen, Programmen und politischen Projekten. Die Zuständigkeit für Devisen, gesperrtes, eingefrorenes und Auslandsvermögen wurde auf die Property Division, OMGUS, übertragen. Selbstverständlich war der Finance Adviser auch für die Entwicklung von Grundsätzen der Geld- und Finanzpolitik zuständig, welche die amerikanische Seite bei BICO betrieb; außerdem vertrat er die Vereinigten Staaten im Finance Directorate der Alliierten Kontrollbehörde und war amerikanisches Mitglied der Allied Bank Commission, die gemäß Militärregierungsgesetz Nr.60 am 15. Februar 1948 als Drei-MächteEinrichtung zur Überwachung der Bank Deutscher Länder geschaffen worden war.

Das Amt des Finance Adviser war in mehrere Functional Groups gegliedert, die im wesentlichen den alten Branches entsprachen: Foreign Exchange Policy unter William S. Balderston, Internal Finance Policy unter Walter Heller, Central Bank unter Henry C. Conrad, Finance Intelligence unter Saul Kagan (später unter Emil Lang) und die Foreign Exchange Depository Group, welche zunăchst weiterhin von Col. William G. Brey, später von Frank J. Roberts in Frankfurt geleitet wurde. Im Februar 1949 wurden die Funktionen der Foreign Exchange Group und der Internal Finance Policy Group in der Internal and External Finance Group unter Ralph McCabe zusammengefaßt.

Die Property Division unter Philip Hawkins gliederte sich in vier Fachunterabteilungen: die wegen Übertragung der Aufgaben auf BICO sehr bald aufgelöste Decartelization Branch unter Richard Bronson, der amerikanisches Mitglied der Decartelization Commission, BICO, wurde; die Property Control and External Assets Branch unter Fred E. Hartzsch; die Reparations Branch unter Orren R. McJunkins und die Restitution Branch unter Col. John H. Allen.

Seit 1948 wurden einige Organisationseinheiten von Berlin in die amerikanische Zone verlegt, so die Property Control and External Assets Branch nach Wiesbaden, die Restitution Branch nach Karlsruhe und die Central Filing Agency (Zentralmeldeamt) unter Bernard Fischbein nach Bad Nauheim.

Durch General Order Nr. 12, OMGUS, vom 7. April 1949 wurde zu einem vergleichsweise sehr späten Zeitpunkt eine Finance Division unter Jo Fisher Freeman wieder errichtet. Mit General Order Nr. 46 vom 3. September 1949 gingen die Zuständigkeiten des Finance Adviser, der Finance Division, des Economics Adviser und des US Chairman of BICO auf ein Provisional Office of Economic Affairs, OMGUS, über. Bei der Auflösung von OMGUS am 21. September 1949 wurde daraus das Office of Economic Affairs, HICOG, das wiederum eine Finance Division und eine Property Division umfaßte.

\section{Armed Forces Division}

Die Armed Forces Division bestand bereits am 9. August 1944 als eine von drei Abteilungen der damals in Bushy Park bei London gegründeten USGCC. Die Abteilung wurde von General John $\mathrm{E}$. Lewis geleitet und war in drei Unterabteilungen (Heer, Marine, Luftwaffe) gegliedert. Ihre Hauptaufgabe bestand in der Vorbereitung der Entwaffnung der deutschen Streitkräfte. 
Durch General Order Nr. 3 vom 25. November 1944 wurden die drei Unterabteilungen mit den Bezeichnungen Military (später Army/Ground) Division unter Brig.Gen. John E. Lewis, Naval Division unter Vice Admiral Robert L. Ghormley und Air Division unter Maj.Gen. Robert W. Harper verselbständigt. Die Umorganisation vom 29. Juni 1945 berührte die genannten drei Abteilungen nicht wesentlich, allerdings erhielt die Army (Ground) Division am 27. Juni mit Maj.Gen. Ray W. Barker einen neuen Leiter. Im Rahmen der Allied Control Authority wurden ein Military (Army), ein Naval und ein Air Directorate geschaffen, in denen die drei Leiter der genannten USGCC-Abteilungen die amerikanischen Interessen wahrzunehmen hatten.

Alle drei Abteilungen verfügten jeweils über eine Demobilization Branch, eine Disarmament und eine Intelligence Branch. Zur Army (Ground) Division gehörte darüber hinaus eine besondere Überwachungsstelle für das Oberkommando der Wehrmacht und das Oberkommando des Heeres; die Besonderheit der Marineabteilung bestand in einer zusätzlichen Ocean Shipping Branch, während die Air Division zwei weitere Unterabteilungen hatte: die Policy and Liaison Branch und eine Operations Branch.

Beim Übergang auf OMGUS wurden die drei „militärischen“ Abteilungen mit Wirkung vom 15. Oktober 1945 unter Maj.Gen. Robert W. Harper erneut zur Armed Forces Division zusammengefaBt. Der Leiter der bisherigen Naval Division, Robert L. Ghormley, wurde durch die General Orders Nr.63 und 67 vom 9. bzw. 26. Oktober 1945 zum Naval Adviser Clays ernannt.

Die neue Armed Forces Division wurde nicht nach Teilstreitkräften, sondern sachsystematisch in zwei große Unterabteilungen gegliedert: die Disarmament Branch unter Col. R. W. Yates und die Demobilization Branch unter Col. T. B. Whitted.

Das Organisationshandbuch vom 9.März 1946 sah zusätzlich zu den beiden genannten eine weitere Unterabteilung vor, die Aeronautios Branch, die ausdrücklich auch für den zivilen Luftverkehr über Deutschland zuständig sein sollte.

Abgesehen von der Umbenennung der Aeronautics Branch in (Civil) Aviation Branch unter Col. G. M. Jones läßt sich für die Jahre 1946/47 eine schrittweise Umstrukturierung der Abteilung nach Angelegenheiten der Teilstreitkräfte beobachten. So gab es im April 1947 eine Army Branch unter Col. W. W. Holler, eine Navy Branch unter Capt. E. J. Burke und eine Air Forces Branch unter Lt.Col. B. E. Steadman.

Aufgrund der General Order Nr. 41 vom 20. Mai 1947 übernahm Col. W. W. Holler geschäftsführend die Nachfolge von Maj.Gen. Robert W. Harper als Abteilungsleiter, wurde jedoch bereits durch General Order Nr.74 vom 1.Oktober 1947 durch Maj.Gen. William E. Hall abgelöst. Nach diesem Revirement wurden die Unterabteilungen für Luftwaffe, zivilen Luftverkehr, Marine und Heer von Lt.Col. D. E. Kunkel, Col. G. M. Jones, Capt. J. S. Crenshaw und Col. T. B. Whitted geleitet.

Im März 1948 kehrte Lt.Col. B. E. Steadman auf seine alte Position als Leiter der Luftwaffenabteilung zurück. Die nach Wiesbaden verlegte Civil Aviation Branch erhielt mit Thomas D. Johnson erstmals einen zivilen Leiter und wurde im Sommer 1948 durch den Aufbau einer Meteorological Branch in Bad Kissingen unter Leitung von Clayton Van Tullenar entlastet. Durch General Order Nr. 38 vom 21. September 1948 wurde Capt. Joseph P. Thew zum Abwesenheitsvertreter des Abteilungsleiters Hall ernannt, der durch General Order Nr.40 endgültig von seinen Pflichten entbunden wurde. Durch General Order Nr. 43 vom 19. Oktober 1948 bestellte man mit Maj.Gen. James P. Hodges einen neuen Direktor der Armed Forces Division, der im Jahre 1949 nur noch drei Unterabteilungen für die Erledigung seiner Aufgaben benötigte, da die drei nach Teilstreitkräften organisierten Unterabteilungen wegen Aufgabenmangels aufgelöst werden konnten. Die restlichen Aufgaben wurden durch General Order 
Nr. 4 vom 4. März 1949 einem Mitte Dezember 1948 eingerichteten Military Security Board übertragen, der für alle drei westlichen Besatzungszonen zuständig war. So bestanden innerhalb der amerikanischen Militärregierung im Jahre 1949 nur noch die Civil Aviation Branch, die Liaison Branch unter Lt.Col. B. E. Steadman und die Meteorological Branch unter H. D. Perry, die im Mai 1949 von Bad Kissingen nach Frankfurt und im Juli desselben Jahres nach Darmstadt verlegt wurde.

Mit der endgültigen Auflösung von OMGUS am 21. September 1949 wurden die Aufgaben der Civil Aviation Branch von der Civil Aviation Division unter Leitung von Thomas D. Johnson im Office of Political Affairs, HICOG, wahrgenommen.

\section{Internal Affairs and Communications Division}

Die Internal Affairs and Communications Division wurde durch die grundlegende Reform von USGCC gemäß General Order Nr.3 vom 25. November 1944 unter der geschäftsführenden Leitung von Brig.Gen. Frank C. Meade geschaffen. Er wurde Ende Mai 1945 kurzfristig in der Leitung der Abteilung von Maj.Gen. Oliver P. Echols abgelöst und ubernahm die Leitung der Communications and Education Branch, zu der damals u. a. auch Religious Affairs gehörten. Außerdem gab es drei weitere Unterabteilungen: Civil Service and Local Government Branch unter Brig.Gen. Luther S. Smith, die Public Safety Branch unter Col. Orlando W. Wilson sowie die Public Health and Welfare Branch unter Maj.Gen. Morrison C. Stayer.

Ende Juni 1945 wurde die Internal Affairs and Communcations Division in vier erheblich kleinere Abteilungen aufgelöst, entstand jedoch mit der Bildung von OMGUS im Oktober 1945 erneut, als das Office of the Assistant Deputy for Public Services, die Communications Division, die Public Safety Division und die Public Health and Welfare Division zu einer Abteilung zusammengefaßt wurden. Die Leitung dieser neuen Abteilung übernahm erneut Brig.Gen. Frank C. Meade. Außer der Civil Administration Branch bestanden im Oktober 1945 wiederum eine Communication Branch unter Brig.Gen. S. M. Thomas, eine Public Safety Branch unter Col. Orlando W. Wilson und eine Public Health and Welfare Branch unter Gen.Maj. Morrison C. Stayer, zu der u. a. eine Education and Religious Affairs Section unter Maj. J. W. Taylor gehörte.

Das Organisationshandbuch vom 9. März 1946 beschreibt die Kompetenzen der Abteilung wie folgt: Gesundheits- und Wohlfahrtswesen, Unterrichts- und Religionsangelegenheiten, öffentliche Sicherheit mit Koordinierungsfunktionen auf dem Gebiet der Entnazifizierung, Allgemeine Innere Verwaltung sowie Post-, Fernmelde- und Nachrichtenwesen. Am 26. Dezember 1946 wurde die Public Health and Welfare Branch in zwei Unterabteilungen gegliedert, wobei der bisherige Unterabteilungsleiter Col. Milford T. Kubin für das Gesundheitswesen verantwortlich blieb, während Alden E. Bevier die Public Welfare Branch übernahm. Die Public Safety Branch, deren organisatorischer Zuschnitt sich nicht änderte, wurde jetzt von Theo E. Hall, die Communications Branch von Eugene H. Merrill geleitet. An die Spitze der Abteilung trat am 17. Mai 1946 Sumner Sewall, der nach weniger als einem halben Jahr am 12. November 1946 von Henry Parkman abgelöst wurde, dem seinerseits bereits am 14. Februar 1947 Dwight P. Griswold folgte. Nach einer Übergangsperiode unter Leitung des Deputy Director, Col. Emil Lenzner, vom 27. Juni bis 25. Juli 1947 wurde die Internal Affairs and Communications Division von George B. McKibbin geleitet, der auch weiterhin Lenzner als Stellvertreter im Amt belieB. Sieht man von personellen Wechseln an der Spitze der Public Welfare Branch - auf Alden E. Bevier folgte Mildred L. C. Biklen, später Mildred B. Smith - einmal ab, änderte sich an der Organisation und 
der personellen Besetzung der Abteilung bis zu deren Auflösung am 1.März 1948 nichts mehr. Die Unterabteilungen Public Safety, Public Health und Public Welfare gingen in der Civil Administration Division auf, während die Ecucation and Religious Affairs Branch von der neuen Education and Cultural Relations Division, die Communications Branch vom Office of the Economics Adviser übernommen wurden.

\section{Civil Administration Division}

Grundsätzlich verstand man in der amerikanischen Armee unter Civil Affairs Division, G-5, den Teil des Stabes, der für Angelegenheiten der zivilen, allgemeinen inneren Verwaltung verantwortlich war. Die am 9. August 1944 in England errichtete USGCC wies zunächst nur drei Abteilungen, die Armed Forces Division und Military Government Division A und Military Government Division B auf. Civil Administration gehörte zur Military Government Division B. Durch General Order Nr.3, USGCC, vom 25. November 1944 entstanden zwölf Fachabteilungen bei USGCC, von denen keine die Bezeichnung Civil Administration Division erhielt; vielmehr wurden die entsprechenden Aufgabenbereiche einer Internal Affairs and Communications Division zugewiesen. An der Spitze von deren Civil Service and Local Government Branch stand Brig.Gen. Luther S. Smith, der am 29. Juni 1945 die damals neu gebildete Civil Administration Division als Abteilungsleiter übernahm. An seinen Kompetenzen änderte sich damals nur wenig, weil an die Stelle der aufgelösten IACD neben der CAD noch drei weitere Abteilungen traten: Communications Division, Public Safety Division und Public Health and Welfare Division.

Durch die umfassende Umorganisation der Militärregierung, d. h. durch die Umwandlung von USGCC in OMGUS, wurde CAD im Oktober 1945 als Unterabteilung in eine Internal Affairs and Communications Division neuer Art eingegliedert. An die Spitze der nunmehrigen Civil Administration Branch trat der spätere Leiter des Regional Government Coordinating Office, James K. Pollock, der gleichzeitig Stellvertreter des Abteilungsleiters war. Pollock wurde am 15. Januar 1946 durch Brig.Gen. Charles K. Gailey als Chief of Branch abgelöst.

Die Unterabteilung gliederte sich in vier Referate: die Administrative Courts Section unter Col. J. A. McComsey, die Local Government Section unter Col. A. F. Hatch, die Civil Service Section unter Lt.Col. H. P. Jones und die Spatial Planning Section unter Col. R. E. Jones. Im Organisationshandbuch vom 9. März 1946 wurden die Zuständigkeiten der Unterabteilung präzise beschrieben: Vereinbarung von Vier-Mächte-Abkommen zur Dezentralisierung der politischen Strukturen in Deutschland, Wiedererrichtung demokratischer Verhältnisse in der kommunalen Selbstverwaltung, Überwachung der politischen Parteien, Vorbereitung zentraler Verwaltungseinrichtungen, Angleichung und Koordinierung der Politik und Verwaltung auf Länderebene sowie Überwachung der Reform des Öffentlichen Dienstes.

Nach der Vereinigung von OMGUS und OMGUSZ erhielt Civil Administration noch im April 1946 wieder den Status einer Abteilung. Unter dem Direktor Henry Parkman, der kurzfristig bereits als Chief of Branch Gaileys Nachfolge angetreten hatte, und dem neuen Deputy Director Edward H. Litchfield wurden - von einem Sekretariat und einer Administrative Branch abgesehen - am 26. April 1946 sechs Unterabteilungen gebildet. 
Ubersicht 38: Organisation und Personal der Civil Administration Division, OMGUS, 26. April 1946

Director (Henry Parkman)

Dep. Director (Edward H. Litchfield)

- Governmental Structures Branch (Kenneth A. Dayton)

- Civil Service Branch (Li.Col. R. P. Rosengren)

- Population and Survey Branch (Karl A. Shafer)

- Administrative Courts Branch (B. D. Sartin)

- Election Affairs Branch (R. M. Scammon)

- Policy Enforcement Branch (Col. M. Boyle)

General Order Nr. 24 vom 26. April 1946 verwies bei der Aufgabenbeschreibung ausdrücklich auf die Kompetenzen der Civil Administration Branch nach der Schilderung im Organisationshandbuch und bezeichnete die darüber hinausgehenden Kompetenzen in folgenden fünf Punkten:

- Allgemeine Aufsicht über die Field Detachments

- Statistik, insbesondere Bevölkerungsentwicklung

- Grundsätze der Aufsicht über deutsche zentrale Einrichtungen

- Fachaufsicht über das Personal im Bereich von Civil Administration bei nachgeordneten Stellen der Militärregierung

- Koordinierung der Tätigkeit aller Teile von OMGUS auf dem Gebiet der Civil Administration auch in Bezug auf die Struktur deutscher Regierungen

Im Frühjahr 1947 wurde durch die Vereinigung der Civil Service Branch und der Administrative Courts Branch die Zahl der Unterabteilungen auf füf verringert. An der Spitze der neuen Civil Service and Administrative Courts Branch stand Ellsworth D. Wolfsperger. Außerdem erhielt die Election Affairs Branch den Namen Election and Political Parties Branch, die Governmental Structures Branch mit Erwin W. Bard ebenso einen neuen Leiter wie die Policy Enforcement Branch mit Col. E. H. Metzger. Politisch besonders bedeutsam war die Übernahme der Leitung der Abteilung durch den vormaligen Stellvertreter des Direktors, Edward H. Litchfield, am 15. März 1947. Der Abteilungsleiter Litchfield wurde durch einen Deputy for Bizonal Affairs, Kenneth A. Dayton, unterstützt, der bisher die Governmental Structures Branch geleitet hatte, die ihrerseits noch im Sommer 1947 den dritten Leiter innerhalb eines Jahres erhielt, Hans Simons. Neu geschaffen wurde die Legislation Branch unter H. Philip Mettger. Auch an der Spitze der Policy Enforcement Branch gab es noch 1947 einen Wechsel: an die Stelle von Erwin W. Bard trat Lt.Col. L. G. Kelly.

Sieht man von der Erweiterung um eine Democratization Branch unter H. W. Landin ab, so blieb diese Besetzung der Civil Administration Division längere Zeit unverändert, bis CAD am 1. März 1948 die Funktionen der Prisoners of War and Displaced Persons Division und einiger Unterabteilung der Internal Affairs and Communications Division (Public Health, Public Welfare, Public Safety) erhielt. Die beiden anderen Abteilungen wurden damals aufgelöst.

Nach dieser Neugliederung bestand die Spitze von CAD aus dem Director's Office unter Edward H. Litchfield, dessen beiden Vertretern, dem Deputy for Bizonal Affairs, Kenneth A. Dayton, und dem Deputy for Operations, H. Philip Mettger. Die nunmehr überdurchschnittlich große und angesichts der einsetzenden Arbeiten des Parlamentarischen Rates zur Schaffung eines Grundgesetzes für die Bundesrepublik Deutschland politisch besonders gewichtige Abteilung gliederte sich - die Personnel and Administration Branch nicht mitgezählt - in nunmehr zehn Unterabteilungen und war 
damit die größte unter allen OMGUS-Abteilungen. An Aufbau und Leitung der fünf bisher schon zur CAD gehörenden Unterabteilungen änderte sich nichts. Die Democratization Branch wurde allerdings in Civil Liberties and Democratization Branch umbenannt. Hinzugetreten waren die Public Safety Branch unter Theo E. Hall, zu der auch die Denazification Section unter Major M. K. Wilson gehörte, ferner die Public Health Branch unter Col. Milford T. Kubin, die Public Welfare Branch unter Mildred B. Smith und schlieBlich die Displaced Persons Branch unter L. G. Kelly, der bisher an der Spitze der aufgelösten Policy Enforcement Branch gestanden hatte.

Wie andere Abteilungen wurde auch CAD in erheblichem Umfang 1948/49 schrittweise von Berlin nach Bad Nauheim und Frankfurt verlegt. Dabei verließen nicht nur Unterabteilungen, sondern auch einzelne Sections die deutsche Hauptstadt. Abteilungsleiter Litchfield und sein Deputy Director for Operations, H. Philip Mettger, blieben in Berlin, während der nun Deputy Director for Bizonia and Governmental Affairs Adviser genannte Kenneth A. Dayton in Frankfurt residierte.

Im April 1949 bestanden schließlich folgende Unterabteilungen der CAD:

Ubersicht 39: Organisation und Personal der Civil Administration Division, OMGUS, April 1949

Director
Edward H. Litchfield (Berlin)
Deputy Director for Operations
H. Philip Mettger (Berlin)
Deputy Director for Bizonia and Governmental Affairs Adviser
Kenneth A. Dayton (Frankfurt)

- Secretariat and Reports Branch unter Alexander R. Forest in Berlin und unter dessen Vertreter William Thad Lovett in Frankfurt

- Governmental Structures Branch unter Hans Simons in Frankfurt

- Legislation Branch unter Leslie Grant in Berlin

- Political Activities Branch unter John Elliot in Frankfurt und unter dessen Vertreter Dale Clark in Berlin

- Population and Analysis Branch unter Karl A. Shafer in Bad Nauheim

- Civil Service and Administrative Courts Branch unter Ellsworth D. Wolfsperger in Frankfurt

- Public Safety Branch unter Theo E. Hall in Berlin und unter dessen Vertreter J. L. McCraw in Bad Nauheim

- Public Health Branch unter Col. Milford T. Kubin in Bad Nauheim

- Public Welfare Branch unter Mildred B. Smith in Bad Nauheim

- Displaced Persons Branch unter L. G. Kelly in Berlin, während das Referat für Flüchtlinge nach Bad Nauheim verlegt wurde

- Civil Liberties and Democratization Branch unter David R. Hunter in Frankfurt

Im September 1949 gingen die Zuständigkeiten der Civil Administration Division, welche neben dem Control Office die Hauptarbeit auf amerikanischer Seite bei der Vorbereitung der Gründung der Bundesrepublik geleistet hatte, im Office of Political Affairs, HICOG, auf, in dem eine Internal Politics and Governmental Division und eine Displaced Populations Division geschaffen wurde.

\section{Education and Cultural Relations Division}

Die Education and Cultural Relations Division wurde gemäß General Order Nr.6, OMGUS, vom 18. Februar 1948 mit Wirkung vom 1. März 1948 errichtet. Sie gehörte damit zu den verhältnismäßig spät gegründeten Abteilungen der amerikanischen 
Militärregierung; ihre Funktionen waren vorher von anderen Verwaltungseinheiten ausgeübt worden.

Die wesentlichen Aufgaben, die später von der Education and Cultural Relations Division wahrgenommen wurden, waren 1944 auf zwei verschiedene Unterabteilungen Education and Religious Affairs verteilt.

Die erste der beiden war Teil der USGCC. Sie zeichnete dort für die Entwicklung von Grundsätzen der amerikanischen Politik auf dem Gebiet von Unterricht und Kultus verantwortlich; außerdem vertrat sie die Vereinigten Staaten in entsprechenden Verhandlungen mit den drei übrigen alliierten Mächten. Im Verwaltungsaufbau von USGCC gehörte die Unterabteilung zur Internal Affairs and Cummunications Division, die zwischen Oktober 1944 und Juli 1945 bestand. Dann wurde die Unterabteilung in die Public Health and Welfare Division überführt, die ihren Abteilungsstatus bei Gründung von OMGUS im Oktober 1945 wieder verlor und Unterabteilung in der Internal Affairs and Communications Division neuer Art wurde. Die Zuständigkeiten für Unterrricht und Kultus bildeten damals eine Section innerhalb der Public Health and Welfare Branch, OMGUS, bis Education and Religious Affairs im Mai 1946 wiederum den Status einer Unterabteilung erhielten.

Die zweite Education and Religious Affairs Branch gehörte zur Civil Affairs Division, G-5, SHAEF. Diese Einrichtung machte praktische Politik über Außenstellen (Field Offices) auf regionaler und lokaler Ebene, wobei man sich der deutschen Verwaltungsstruktur (Länder, Regierungsbezirke, Stadt- und Landkreise) anpaßte. Nach der Überführung von SHAEF in USFET im Juli 1945 spielte die Unterabteilung eine führende Rolle bei der Durchfühung der amerikanischen Besatzungspolitik im Rahmen ihrer Zuständigkeit und überwachte entsprechende deutsche Behörden. Die USFETEinheit wurde im Oktober 1945 von einer OMGUSZ-Dienststelle mit Sitz in Frankfurt abgelöst.

Erst nach Überfuhrung der Frankfurter Stelle nach Berlin am 2. Mai 1946 wurden planerische und durchfuhrende Aufgaben in einer einzelnen Education and Religious Affairs Branch, OMGUS, unter John W. Taylor, seit Ende 1946 unter Richard T. Alexander zusammengefaBt, welche Teil der Internal Affairs and Communications Division, OMGUS, blieb, bis die Education and Cultural Relations Division zum 1. März 1948 geschaffen wurde. Damit kam deutlich ein wachsendes Interesse der Besatzungsverwaltung an kulturellen und religiösen Fragen zum Ausdruck. Dies wird nicht zuletzt durch die Schaffung eines besonderen Reorientation Funds sichtbar. Geschäftsführender Leiter der Abteilung wurde am 1. März 1948 Herman B. Wells, der am 23. Juli desselben Jahres von Alonzo G. Grace abgelöst wurde.

Neben dem Director's Office bestanden Anfang 1948 Fachunterabteilungen für Erziehung (Education Branch) unter Richard T. Alexander, 1949 unter John O. Riedl, für religiöse Angelegenheiten (Religious Affairs Branch) unter C. Arild Olsen, für Jugend, Frauen und Erwachsenenbildung (Group Activities bzw. Community Education Branch) unter Lawrence E. Norrie und für Kulturaustausch (Cultural Exchange Branch) unter Frank G. Banta. Die letztgenannte Unterabteilung war vor allen Dingen verantwortlich für die Vorbereitung und Durchführung von Austauschprogrammen für Wissenschaftler, Studenten, Verwaltungsbeamte und sogenannte Community Leaders im Rahmen eines German Experts Program, um in den Vereinigten Staaten demokratische Einrichtungen und Verfahren zu studieren sowie moderne Techniken auf dem jeweiligen Spezialgebiet kennenzulernen. Vergleichbare Programme brachten umgekehrt auch amerikanische Experten nach Deutschland, wo sie die Militärregierung berieten und mit fachlich interessierten deutschen Stellen zusammenarbeiteten. 
Im Juni 1948 wurde die Cultural Exchange Branch zur Cultural Affairs Branch umgewandelt, als mehrere Funktionen, die bisher von der Unterabteilung für Theater und Musik, Information Services Division, wahrgenommen worden waren, dieser Unterabteilung zugewiesen wurden. Durch General Order Nr. 29 vom 26. Juli 1948 wurde ein Inter-Divisional Reorientation Committee gegrlindet, das unter Leitung des Direktors der Education and Cultural Relations Division aus je einem Mitglied der Civil Administration Division, der Information Control Division und des Control Office sowie der Cultural Exchange Branch als Sekretär bestand. Um die Jahreswende 1948/49 wurden auch die Aufgaben der Monuments, Fine Arts and Archives Section aus der Restitution Branch der Property Division in die Cultural Affairs Branch uberführt, die damals von Ralph A. Burns geleitet wurde. Schließlich erhielt die Abteilung auch im September 1949 noch einen Kompetenzzuwachs, als sie die Information Centers Branch übernahm, die vorher zur Information Services Division gehört hatte.

Ursprünglich war die Education and Cultural Relations Division in Berlin errichtet worden, wurde jedoch im Sommer 1948 nach Nürnberg in den Justizpalast verlegt, wo sie bis März 1949 blieb, als sie zusammen mit dem Deputy Chief of Staff nach Bad Nauheim umzog. Bei der Übernahme von OMGUS durch HICOG ging die Education and Cultural Relations Division gemäß General Order Nr. 44 vom 3. September 1949 im Provisional Office of Public Affairs, OMGUS, bzw. Office of Public Affairs, HICOG, auf.

\section{Prisoners of War and Displaced Persons Division}

Die Prisoners of War and Displaced Persons Division, USGCC, wurde durch General Order Nr. 3 vom 25. November 1944 unter Leitung von Brig.Gen. Eric F. Wood geschaffen. Sie hatte bei ihrer Arbeit auch nach der Errichtung von OMGUS stets erhebliche Reibungsverluste gegenüber entsprechenden Kompetenzen von G-5, SHAEF, USFET und EUCOM. Die Umorganisation von USGCC Ende Juni 1945 berührte die Abteilung dagegen nur insoweit, als sie mit Brig.Gen. Stanley R. Mickelsen einen neuen Leiter erhielt, der auch nach der Errichtung von OMGUS im Oktober $1945 \mathrm{im}$ Amt blieb. Bereits im Juni 1945 gab es die beiden Unterabteilungen Displaced Persons Branch unter Col. H. W. Anderson und Prisoners of War Branch unter Lt.Col. C. H. Ott. Mit dem Übergang auf OMGUS trat im Bereich der Unterabteilungen nur insoweit eine Änderung ein, als Lt.Col. James P. Abbott an die Stelle von Col. Anderson trat. Das Organisationshandbuch vom 9.März 1946 unterstreicht die Kompetenzüberschneidungen zwischen OMGUS und USFET mit Nachdruck, die auch durch die Auflösung von OMGUSZ am 1. April 1946 nicht entwirrt wurden.

Das Bild änderte sich im Jahre 1947 dadurch, daß nunmehr drei OMGUS-Unterabteilungen unter folgenden Bezeichnungen und Leitern bestanden: Displaced Persons and Stateless Persons Branch unter Lt.Col. James P. Abbott, Refugees and United Nations Prisoners of War Branch unter Lt.Col. Charles E. Boyle sowie Repatriation Branch unter Maj. John F. Asselta. Im September 1947 übernahm Brig.Gen. Thomas L. Harrold die Leitung der Abteilung mit Sitz in Frankfurt. Da er gleichzeitig Leiter der Civil Affairs Division, EUCOM, war, wurden die Reibungsverluste zwischen OMGUS und EUCOM auf diese Weise wahrscheinlich gemildert. In Berlin wurde Lt.Col. Harry S. Messec mit Wirkung vom 5. November 1947 zum Deputy Director bestellt. Bis zur Auflösung der Abteilung mit Ablauf des 28. Februar 1948 waren organisatorische oder personelle Veränderungen nicht mehr festzustellen. Ihre Aufgaben gingen auf die Civil Administration Division über. 


\section{Information Services Division}

Der Vorgänger von OMGUS - USGCC - verfügte aufgrund General Order, ETOUSA, vom 15. März 1945, also bereits vor der Auflösung von SHAEF, über einen Information Control Service. Leiter dieser Einheit wurde gleichzeitig Brig.Gen. Robert A. McClure. Unter Information Control verstand man die Überwachung der Entnazifizierung, Entmilitarisierung und Umerziehung des deutschen Volkes sowie die Lizenzierung der deutschen Informationsdienste (Bücher, Zeitungen, Zeitschriften, wissenschaftliche Veröffentlichungen $u$. a. m.); hinzu traten Überwachungsfunktionen bei der Herstellung, Synchronisierung und Verbreitung von Filmen sowie im Bereich von Theater, Musik und Rundfunk. Bei SHAEF waren die Psychological Warfare Division und das Office of War Information für die Planung der Besatzungspolitik auf den genannten Gebieten zuständig. Als SHAEF Mitte Juli 1945 aufgelöst wurde und britische bzw. amerikanische Einrichtungen an seine Stelle traten, wurde eine Information Control Division bei USFET in Frankfurt zur Durchführung entsprechender Aufgaben und ein Office of the Director of Information Control (Information Services Control) im Rahmen der USGCC in Berlin als eine für politische Grundsätze zuständige Koordinierungsstelle errichtet. Der Leiter von ODIC, Brig.Gen. Robert A. McClure, vertrat die Vereinigten Staaten auch im entsprechenden AusschuB des Political Directorate der alliierten Kontrollbehörde, die niemals ein eigenständiges Information Control Directorate errichtete.

Bei der Errichtung von OMGUS faßte man alle Zuständigkeiten unter dem Director of Information Control zusammen. Sein Office umfaBte im Oktober 1945 insgesamt 15 Mitarbeiter und gliederte sich in einen Stab des Direktors McClure und drei - alle von Zivilbediensteten geleitete - Sections: die Intelligence Section unter A. Thoombs, die Publishing Operations Section unter E. N. Reinsel und die Radio Section.

Mit Inkrafttreten des Organisationshandbuches vom 9. Mărz 1946 wurde das Office of the Director of Information Control erheblich ausgebaut. Die Staff Group war für die politische Koordinierung verantwortlich; außerdem bestanden fünf Branches für die Überwachung der Presse, für die Kontrolle anderer Publikationen, für den Rundfunk, für Film, Theater und Musik sowie für Business Management. Unter Business Management verstand man das wirtschaftliche und finanzielle Gebaren der amerikanischen wie deutschen Informationsdienste, insbesondere auch die Beschaffung von Papier. Mit der Auflösung von OMGUSZ im April 1946 gingen die Funktionen der dortigen Information Control Division auf OMGUS in Berlin über. Nunmehr gab es hier ein Information Control Office. ICD wies 1946 folgende personelle Besetzung auf: Das Office of the Director wurde weiterhin von Brig.Gen. Robert A. McClure geleitet, die Plans and Directives Branch von Alfred V. Boerner, die Business Management Branch von John Kidder, die Intelligence Branch von Robert C. Schmid, die Publications Control Branch von Lt.Col. Douglas Waples, die Radio Control Branch von Charles S. Lewis, die Press Control Branch von Arthur D. Eggleston und die Film, Theater and Music Control Branch von Eric T. Clarke. In den folgenden Jahren änderte OMGUS diese Verwaltungsstruktur mehrfach. Durch General Order Nr. 16, OMGUS, vom 7. März 1947 wurde aus dem Office die Information Control Division. Der Name der Intelligence Branch wurde - ebenfalls 1947 - in Research Branch geändert; eine Information Center Exhibition Branch unter William C. Headrick, später unter Patricia Van Delden, trat hinzu.

An der Spitze der Abteilung wurde Brig.Gen. McClure am 15. Mai 1947 durch Col. Gordon E. Textor abgelöst. Im Jahre 1949 trat eine Overt Management Branch unter Lt.Col. Donald T. Jones an die Stelle der Business Management Branch; ein stellver- 
tretender Abteilungsleiter für Politik und multinationale Angelegenheiten, Alfred V. Boerner, wurde zusätzlich zu dem aligemeinen, am 27. Juli 1947 ernannten Stellvertreter des Abteilungsleiters, Thomas P. Headen, berufen, um sowohl die Plans and Directives Branch, an deren Spitze Herbert C. Mayer auf Alfred V. Boerner folgte, als auch die neu geschaffene Political Information Branch unter W. B. Phillips, später unter Lawrence F. Babcock, koordinierend zu überwachen. Das besser gewordene Verhältnis zwischen Besatzern und Besetzten kam im August 1948 dadurch zum Ausdruck, daß der Name der Abteilung unter Streichung des Wortes "Control“ in Information Services Division geändert, das Personal erheblich vermindert wurde und die Tàtigkeit der Abteilung eher in der Abgabe von Empfehlungen an deutsche Stellen als in unmittelbarer Überwachung bestand.

Im Juni 1948 wurde die Theater and Music Section an die neu geschaffene Education and Cultural Relations Division abgegeben, so daß bei ISD lediglich eine Motion Picture Branch zurückblieb, die nach München verlegt wurde. Im Dezember 1948 änderte sich die Bezeichnung der Plans and Directives Branch in Policy and Programming Branch; außerdem wurden die Business Management und die Overt Management Branch in der Fiscal and Budget Control Branch zusammengefaßt. Die Research Branch wurde durch die Opinion Surveys Branch ersetzt, die Publishing Operations Branch übernahm die Zuständigkeiten für Veröffentlichungen der Militärregierung. während die Editorial Projection Branch für Grundsatzfragen der Überwachung deutscher Publikationen mit dem Ziel der Durchführung der amerikanischen politischen Grundsätze verantwortlich wurde; dazu gehörte auch die Besorgung von Material für das bewuBt antikummunistische Vigorous Information Program.

Im April 1949 gliederte sich die Abteilung in zehn Unterabteilungen, die in fünf verschiedenen Städten ihren Dienstsitz hatten. Dieser Umstand wird kaum als DezentralisierungsprozeB verstanden werden können; er ist offensichtlich darauf zurückzuführen, daß die Besatzungsmacht am Ort der entsprechenden deutschen Fachverwaltung präsent sein wollte.

Ubersicht 40: Organisation und Personal von Information Services Division, OMGUS, April 1949

- Director's Office (Col. Gordon E. Textor) in Berlin

- Deputy Director (Thomas P. Headen) in Bad Nauheim

- Policy and Programming Branch (Alfred V. Boemer) in Berlin

- Fiscal and Budget Control Branch (Daniel C. Mulloney) in Berlin

- Opinion Surveys Branch (Leo P. Crespi) in Bad Nauheim)

- Editorial Projection Branch (John M. Stuart) in Frankfurt

- US Feature Service (Charles B. Amot) in Frankfurt

- Information Centers and Exhibitions Branch (Patricia Van Delden) in Bad Nauheim

- Motion Picture Branch (Arthur Mayer) in München

- Radio Branch (Charles S. Lewis) in Berlin

- Press Branch (Arthur D. Eggleston) in Bad Nauheim

- Publications Branch (Lawrence P. Dalcher) in Bad Nauheim

- Publishing Operations Branch (Kendall Foss) in München

Beim Übergang von OMGUS zu HICOG im September 1949 wurde Information Services Division Bestandteil des Office of Public Affairs, HICOG. 


\section{Legal Division}

Die anfangs bei USGCC für Rechtsfragen zuständige Military Government Division A wurde durch General Order Nr. 3 vom 25. November 1944 aufgelöst, eine selbständige Legal Division unter der geschäftsführenden Leitung von Lt.Col. J. B. Marsh gegründet. Ihre Aufgaben bestanden damals vor allem in der Rechtsberatung und der Planung der Rechtspolitik, der Vorbereitung des Aufbaus von Gerichten der Militärregierung und der Überwachung der deutschen Justiz sowohl im zivil- als auch im strafrechtlichen Bereich. Dementsprechend verfügte die Abteilung über folgende Unterabteilungen.

Übersicht 41: Organisation und Personal der Legal Division, USGCC, 25. November 1944

Director Lt.Col. J. B. Marsh

- Legal Advice Branch (Col. John M. Raymond, später Lt.Col. S. Bradford)

- Justice Ministry Branch (Col. E. L. McLendon)

- Judicial Branch (Maj. D. F. White, später Lt.Col. Frank Seydel)

- Prisons Branch (Col. A. B. Michell)

Durch die beiden grundlegenden Orders Nr.33 und Nr.34 vom 29. Juni 1945 erhielt Charles Fahy die Funktion eines Legal Adviser im damals eingerichteten Executive Office, während die Legal Division geschäftsführend von Brig.Gen. Edward C. Betts übernommen wurde.

Als OMGUS am 1.Oktober 1945 an die Stelle von USGCC trat, wurden die Funktionen der vier Unterabteilungen nur geringfügig geändert. Anders verhielt es sich auf der Führungsebene. Während die Leitung der Legal Division schon seit dem 20. Juli 1945 vom Legal Adviser, Charles Fahy, in Personalunion ubernommen worden war, wurden der bisherige geschäftsfuhrende Direktor, Brig.Gen. Edward C. Betts zum Deputy Director for War Crimes und Col. J. B. Marsh zum Deputy Director for Administration, beide außerhalb der Fachabteilungen, ernannt.

Die Legal Advice Branch beriet die Militärregierung in allen Fragen, die amerikanisches Recht, die Umsetzung der Politik der Militärregierung in Rechtsvorschriften, deutsche Gesetze, Völkerrecht und andere politische Fragen rechtlicher Relevanz betrafen; hier galt es vor allem, entsprechende Rechtsvorschriften zu entwerfen und die Entwürfe anderer Abteilungen auf Rechtsförmlichkeit zu überprüfen. Die Administration of Justice Branch als ZusammenschluB der früheren Justice Ministry Branch und der Judicial Branch war seit 1946 dafür verantwortlich, Studien und Pläne zu verfassen, die sich mit der Wiedererrichtung deutscher Justizministerien auf zentraler bzw. Landesebene ebenso beschäftigten wie mit dem Aufbau der Justizbehörden und Gerichte einschließlich der Verwaltungsgerichtsbarkeit. Die Prisons Branch war für die Entwicklung von politischen Grundsätzen für die Überwachung der deutschen Gefängnisse verantwortlich. Von besonderer Bedeutung erwies sich dann ab 1946 die War Crimes Branch, die für die Grundsätze der amerikanischen Politik auf dem Gebiet der Verfolgung von Kriegsverbrechern, deren Auslieferung bzw. deren Anklage und Bestrafung verantwortlich war. Außerdem hatte die Verfolgung der Kriegsverbrecher nicht nur zur Bildung einer Unterabteilung War Crimes, sondern auch eines von der Abteilung unabhängigen Office of US Chief Counsel for War Crimes geführt.

Am 15. Mai 1946 war der Berufsrichter J. Warren Madden zum Legal Adviser und Leiter der Rechtsabteilung ernannt worden; gleichzeitig wurde Alvin J. Rockwell die bisher von Madden wahrgenommene Stelle eines Associate Director der Rechtsabtei- 
lung übertragen. Bereits am 25. Juni 1946 trat Rockwell die Nachfolge Maddens als Leiter der Abteilung an. Nachfolger Rockwells als Associate Director wurde Oberst John M. Raymond am 20. August 1946. Die Errichtung des Länderrats als eines deutschen zonalen Organs unter Aufsicht der Militärregierung und dessen ständig zunehmende Kompetenzen auf dem Gebiet der Gesetzgebung machten im Oktober 1946 die Errichtung einer fünften Unterabteilung unabweisbar erforderlich: der Legislation Branch.

Durch General Order Nr. 3 vom 17. Januar 1947 wurde bekannt gegeben, daB mit Wirkung vom 1.Oktober 1946 ein Board of Clemency errichtet worden sei, dem mindestens drei weitere Mitglieder angehörten; dieses Gremium hatte Vorschläge für Begnadigungsentscheidungen zu erarbeiten. Durch General Order Nr.26 vom 16. Juli 1948 wurde dieser Board aufgelöst.

Durch General Order Nr.29 vom 2. April 1947 setzte man einen Extradition Board ein, dem unter dem Vorsitz des Vertreters der Rechtsabteilung je ein Beauftragter des Office of Director of Political Affairs, des Office of Director of Intelligence und der Internal Affairs and Communications Division angehörten. Durch die General Orders Nr. 30, 33 und 61 vom 4., 9. April bzw. 26. Juli 1947 wurde ein Legislation Review Board errichtet, in den man im April unter dem Leiter der Rechtsabteilung fünf weitere Mitglieder - zum Teil alternierend - persönlich berufen hatte; dagegen wurde im Juli entschieden, daB - unverändert unter dem Leiter der Rechtsabteilung - die Leiter der Civil Administration Division und der Internal Affairs and Communications Division sowie ein Vertreter des Office of the Chief of Staff je einen Sitz in dem Gremium einzunehmen hätten. Die Abteilungsleiter waren befugt, sich vertreten zu lassen. Durch General Order Nr. 15 vom 17. April 1948 übertrug man den Vorsitz im Legislation Review Board dem Leiter der Civil Administration Division; Mitglied des Board blieben oder wurden der Leiter der Rechtsabteilung oder sein Beauftragter, der Governmental Administration Adviser und der Vertreter des Office of Chief of Staff. Durch General Order Nr. 42 vom 15. Oktober 1948 wurde der Sitz des Board von Berlin nach Nümberg verlegt.

Im Verlaufe des Jahres 1947 ergaben sich keine wesentlichen organisatorischen Veränderungen in der Rechtsabteilung der Militärregierung. Nach dem Stand vom September 1947 wurde die Legal Advice Branch von William E. McCurdy als Nachfolger von B. G. Habberton, die Legislation Branch von E. H. Schopler als Nachfolger von Samuel Kramer, die Administration of Justice Branch von Haven Parker, die Prisons Branch von T. Wade Markley als Nachfolger von S. Sauter und die War Crimes Branch von Ben Smith geleitet. Hinzu trat als Vorsitzender des Board of Clemency J. J. Walsh.

Col. John M. Raymond wurde am 15. März 1948 Nachfolger Rockwells an der Spitze der Rechtsabteilung, während Whitney R. Harris an die Stelle Raymonds trat. Charles H. Kraus übernahm die nach Nürnberg verlegte Administration of Justice Branch. Dort taten damals auch bereits die Prisons Branch und die War Crimes Branch ihren Dienst. Die beiden in Berlin verbliebenen Unterabteilungen hatten ebenfalls neue Leiter erhalten: die Legal Advice Branch James E. Heath und die Legislation Branch Creighton R. Coleman.

Durch General Order Nr. 32 vom 18. August 1948 wurden in Durchführung der Military Government Ordinance Nr.31 „Military Government Courts for Germany“ mit einem Chief Judge of the Court of Appeals errichtet. Die Dienstaufsicht über diese Militärregierungsgerichte übte der Deputy Military Governor, Maj.Gen. George P. Hays, durch den Chief of Staff, OMGUS, aus. Die Anklagevertretung übernahm ein Office of the Chief Attomey, das im Rahmen der Legal Division errichtet wurde. 
Mit Abschluß der Nürnberger Prozesse wurde die War Crimes Branch aufgelöst. Während sich der organisatorische Zuschnitt bis September 1949 nicht mehr änderte. ergaben sich in der Rechtsabteilung noch einige räumliche Verlagerungen. Sie verfügte 1949 nicht mehr über Dienststellen in Nürnberg. Vielmehr wurde die Administration of Justice Branch unter Mortimer Kollender ebenso nach Bad Nauheim verlegt wie die Prisons Branch unter Sidney H. Souter. Die beiden übrigen Unterabteilungen blieben in Berlin, wobei die Legislation Branch mit John J. Barron einen neuen Leiter erhielt. Anstelle von Col. John M. Raymond übernahm am 28. März 1949 der bisherige Associate Director, William E. McCurdy, die Leitung der Abteilung.

Wie auch bei anderen Verwaltungseinheiten üblich, ging die Legal Division unmittelbar vor der Auflsöung von OMGUS gemäß General Order Nr. 45 vom 3. September 1949 in ein Provisional Office, OMGUS, uber, das den Namen Office of General Counsel erhielt und diese Bezeichnung ab 21.September 1949 als HICOG-Einrichtung fortfuihrte.

\section{Office of the Chief of Counsel for War Crimes / Military Tribunals}

Vorgänger des Office of the Chief of Counsel for War Crimes war das Office of the United States Chief of Counsel, das die amerikanische Anklagebehörde beim Internationalen Militärtribunal in Nünberg bildete. Im Januar 1946 wurde sie der Kommandogewalt von OMGUS unterstellt. Zusätzlich zu ihren bisherigen Aufgaben wurde ihr auch die Verwaltung der amerikanischen Militärgerichte (Military Tribunals) einschlieBlich des inneren Dienstes bei den zwölf sogenannten Nachfolgeprozessen in Nürnberg übertragen. Hauptaufgabe des Office of the Chief of Counsel for War Crimes war die Feststellung. Gefangennahme und Verfolgung von deutschen Kriegsverbrechem im amerikanischen Besatzungsgebiet gemäß Kontrollratsgesetz Nr. 10.

Der Chief of Counsel hatte ebenso wie der ganz überwiegende Teil der Verwaltung des OCCWC seinen Dienstsitz in Nürnberg; in Berlin wurde lediglich eine kleine Berlin Branch unterhalten, deren Referate sich 1947 mit der Sicherung von Beweismaterialien in den Komplexen SS, Wirtschaft und Reichsregierung (Ministerien) beschäftigten. In Nürnberg befanden sich 1948 neben dem Chief of Counsel, Brig.Gen. Telford C. Taylor, und mehreren Deputy Chiefs of Counsel für den I. G. Farben-Prozeß (Josiah E. Dubois), für den Krupp-Prozeß (Rawlings Ragland), für den Weizsäkker-Prozeß (Robert M. W. Kempner) und für den OKW-Prozeß (James M. McHaney) folgende Abteilungen:

Ubersicht 42: Organisation und Personal des Office of the Chief of Counsel for War Crimes, OMGUS, 1948

- Administrative Division (Capt. Donald Paul)

- Reproduction Division (Maj. Alexander C. Granzin)

- Signal Division (Maj. Kenneth K. Evans)

- Language Division (Thomas K. Hodges)

- SS and Military Division (James M. McHaney)

- Evidence Division (Walter H. Rapp)

- Economic Ministries Division (Robert M. W. Kempner)

- Special Projects Division (Paul H. Gantt)

- Public Relations Division (Ernest D. Deane)

Im Frühjahr 1949 war die Zahl der Abteilungen auf sieben reduziert. 
Ubersicht 43: Organisation und Personal des Office of the Chief of Counsel for War Crimes, OMGUS, Frühjahr 1949

- Document Disposal Division (Fred Niebergall)

- Economic Ministries Division (Morris Amchan)

- Political Ministries Division (Robert M. W. Kempner)

- Publications Division (Drexel A. Sprecher)

- Publications Division (German Edition) (Paul H. Gantt)

- Public Relations Division (Eugene Philips)

- Reproduction Division (Erich G. R. Rehberg)

Der Internationale Militärgerichtshof hatte sich im Oktober 1945 Verfahrensvorschriften gegeben, die u. a. der Errichtung eines Sekretariats unter einem Generalsekretär dienten. Die Aufgaben des IMT-Sekretariats wurden bei den amerikanischen Militärgerichten fortgeführt und durch die Verordnung Nr. 7 der Militärregierung über die Organisation und Vollmachten bestimmter Militärgerichte vom 18. Oktober 1946 geregelt. Durch die General Order Nr. 67, OMGUS, vom 25. Oktober 1946 wurde das Sekretariat der Militärtribunale OMGUS in Berlin unterstellt, dort berichtete aus Gründen der Verwaltungsvereinfachung der Generalsekretär zunächst OCCWC in Nürnberg. Als Secretary General for Military Tribunals wurde aufgrund der General Order Nr. 75 vom 13. November 1946 George M. Read als Nachfolger von Charles E. Sands ernannt, der jedoch vom 24. Januar bis 19. April 1947 dieses Amt erneut bekleidete, ihm folgte Col. John E. Ray. Am 10. Mai 1948 übernahm Howard L. Russell das Generalsekretariat und leitete es bis über die Auflösung der OMGUS-Verwaltung hinaus. Die Military Tribunals befanden sich alle in Nürnberg und behandelten die sogenannten Nachfolgeprozesse, wobei die Nummernfolge auf die Chronologie der Errichtung schließen läßt.

\section{Manpower Division}

Bei der Gründung von USGCC gehörte der Bereich Arbeit und Soziales (Manpower) zur Military Government Division B. Im November 1944 entstand dann die Manpower Division, USGCC, unter Maj. David A. Morse. Im Juni 1945 unterstand sie der Aufsicht des Assistant Deputy for Public Services und umfaßte unter der Leitung von Brig.Gen. Frank J. McSherry folgende Unterabteilungen:

Ubersicht 44: Organisation und Personal der Manpower Division, USGCC, Juni 1945

\section{Director Brig.Gen. Frank J. McSherry}

- Administrative Office (J. H. Chadwick)

- Labor Ministry Section

- Labor Intelligence Section

- Nazi Labor Instituts Section

- Labor Standards and Social Insurance Branch (P. R. Porter)

- Labor Allocations Branch (Lt.Col. A. H. Aronson)

- Housing Branch (Maj. D. Snyder)

Nach der Umorganisation von USGCC zu OMGUS gab es neben dem Amt des Directors acht Referate: 
Übersicht 45: Organisation der Manpower Division, OMGUS, Juli 1945

\author{
Planning Section \\ - Labor Allocation Section \\ - Intelligence Section \\ - Labor Relations Section \\ - Housing Section \\ - Labor Institutions Section \\ - Social Insurance Section \\ - Reports and Statistics Section
}

Leiter der Manpower Division blieb Brig.Gen. Frank J. McSherry. Doch bald wurde das System der Branches wieder eingeführt.

Das Organisationshandbuch vom 15. März 1946 sah sechs Unterabteilungen vor, von denen die Labor Relations Branch mit Kompetenzen auf dem Gebiet der Gewerkschaften, der Arbeitskämpfe einschlieBlich Schlichtung und Schiedssprüche, der Arbeitsgerichte sowie der Tarifvertragsverhandlungen und die Housing Branch (Wohnungsbau) ihre alten Namen weiterführten. Die frühere Labor Standards and Social Insurance Branch wurde durch zwei selbständige Unterabteilungen ersetzt, wobei die Labor Standards Branch für Löhne und Einkommen, Arbeitszeit und -bedingungen sowie Arbeiterschutzgesetzgebungen und die Social Insurance Branch für alle Arten der Sozialversicherungen zuständig waren. Die Labor Allocations Branch, USGCC, hieB nunmehr Manpower Allocations Branch, OMGUS, und war für den Arbeitseinsatz sowie Beschäftigungs- und Arbeitslosenfragen verantwortlich. Wirklich neu war also nur die Field Operations Branch, welche die Grundsätze der Politik von OMGUS und die daraufhin entwickelten Programme auf regionaler und lokaler Ebene uberwachte und förderte. Die Leitung der Abteilung übernahm im Juni 1946 Leo R. Werts. Unter ihm bestanden neben den drei Staff Branches (Stabsunterabteilungen) in den Jahren 1946/47 vier Unterabteilungen: Labor Relations unter Maj. Harold F. Mullaney, Wages and Labor Standards unter Sol Ozer, Social Insurance unter M. G. Murray und Manpower Allocation unter George S. Wheeler.

Mitte 1948 verfugten die Wages and Labor Standards Branch mit Earl R. Beckner und die Labor Relations Branch mit John K. Meskimen über neue Leiter, während alle anderen Kompetenzen in der Reports and Statistics Branch unter Albert H. Berman zusammengefaßt waren.

Im März 1949 gab es dann wieder vier Unterabteilungen: Labor Information and Orientation Branch unter Nels Anderson, Labor Economics Branch unter Earl R. Beckner, Labor Relations unter John K. Meskimen und die Research and Analysis Branch unter Albert H. Berman; gleichzeitig hatte der Leiter des Sekretariats der Abteilung den Rang eines Unterabteilungsleiters. An dieser Organisation änderte sich bis zur Gründung von HICOG nichts mehr, wobei die Kompetenzen von Manpower Division, OMGUS, in das Office of Labor Affairs (Labor Division / Manpower Division), HICOG, zusammengeführt wurden.

\title{
21. Transport Division
}

Bei der Gründung von USGCC wurde der Bereich Verkehr (Transport) von der Military Government Division A wahrgenommen. Durch General Order Nr. 3, USGCC, schuf man dann eine Transport Division unter Col. J. A. Appleton. Nach Einführung der neuen Organisationsstruktur Ende Juni 1945 wurde die Transport Division dem 
Assistant Deputy for Public Services unterstellt und gliederte sich in folgende Unterabteilungen:

Úbersicht 46: Organisation und Personal der Transport Division. USGCC, Juni 1945

- Inland Waterways Branch (Li.Col. H. A. Knoll)

Director Col. J. A. Appleton

- Movement and Traffic Branch (Lt.Col. E. B. McKinney, später Col. T. C. Wenzlaff)

- Planning Branch (Lt.Col. H. H. Yokes)

- Ports and Coastal Shipping (Comdr. F. H. Foster)

- Rail Branch (Lt.Col. W. G. White)

- Road Branch (Lt.Col. J. L. S. Snead)

Durch zwei USFET-Direktiven vom Juli und September 1945 wurde eine Road Transportation Section und eine Movements Sub-Section in der Economics Branch, G-5, USFET, errichtet. Die Umwandlung von USGCC in OMGUS und G-5, USFET, in OMGUSZ zum 1. bzw. 15. Oktober 1945 berührte die Verkehrsverwaltung nur unwesentlich; so wurden bei OMGUS die Inland Waterways Branch und die Ports and Coastal Shipping Branch zur Water Transport Branch unter Lt.Col. D. R. Neff zusammengefaßt. Nach der Vereinigung von OMGUS und OMGUSZ am 1. April 1946 gab es neben der Executive Branch eine Movements (and Traffic) Branch unter J. C. Cook, eine Rail Branch unter E. V. Breitenbach, eine Water (Transport) Branch unter D. R. Neff und eine Road Branch unter C. E. Calvert. Der Abteilungsleiter - ab 12. November 1946 geschäftsführend, ab 10. November 1947 endgültig Col. Hans W. Holmer - und dessen Stellvertreter nahmen die amerikanischen Interessen im Transport Directorate der Alliierten Kontrollbehörde wahr.

Die Militärregierung unterhielt eigene nachgeordnete Behörden für die WasserstraBenverwaltung, die mit den deutschen Wasser- und Schiffahrtsdirektionen vergleichbar sind. Das Rhine Field Office, das Danube Field Office in Passau und das Bremen Field Office waren - für Bremen gilt dies nur mit gewissen Einschränkungen - nicht den jeweiligen Landesmilitärregierungen zugeordnet, sondern erstatteten unmittelbar Bericht an den Leiter der Unterabteilung für Schiffahrt in der Verkehrsabteilung von OMGUS. Hinzu kamen Inspection and Security Sections, die gegenüber den deutschen Wasser- und Schiffahrtsbehörden die Einhaltung der Weisungen der Militärregierung im Bereich der deutschen Binnenschiffahrt zu überwachen, die deutschen Binnenschiffahrtsflotte zu erfassen und Genehmigungen für die Binnenschiffahrt zu erteilen hatten. Außerdem gab es ein Rail Field Office mit entsprechenden Funktionen für die Überwachung der deutschen Eisenbahnen.

Im Zusammenhang mit der wirtschaftlichen Vereinigung der amerikanischen und der britischen Zone am 1. Januar 1947 wurde im Bipartite Control Office eine Transport Group errichtet, die auf amerikanischer Seite von Col. A. H. Schroeder geleitet wurde. Durch General Order Nr. 18, EUCOM, löste man mit Wirkung vom 1. März 1948 die Transport Division auf und ubertrug deren Aufgaben, soweit sie nicht auf BICO oder deutsche bizonale Stellen übergingen, dem Office of the Economics Adviser.

\section{Regional Government Coordinating Office}

Der Aufbau des Regional Govemment Coordinating Office begann am 26. Oktober 1945 in Stuttgart. Das Amt diente der Militärregierung als koordinierende Dienststelle in allen Fragen, die mehr als ein deutsches Land in der amerikanischen Zone betra- 
fen. Weisungen von OMGUS an die deutschen Landesregierungen liefen grundsätzlich über das Regional Government Coordinating Office; für den amerikanischen Sektor von Berlin war es nicht zuständig.

Durch General Order Nr.21 vom 18. April 1946 wurde James K. Pollock förmlich zum Direktor des RGCO bestellt. Vorher hatte er seine Tätigkeit aufgrund mündlicher Weisungen durchgeführt. Die Dienststellenverwaltung des RGCO wurde vom Office of Military Government for Württemberg-Baden wahrgenommen.

Pollock war als Leiter des RGCO dem Militärgouverneur bzw. seinem Stellvertreter unmittelbar verantwortlich. Weisungen der Fachämter oder Fachabteilungen von OMGUS hatten er und seine insgesamt geringe Mitarbeiterzahl nicht entgegenzunehmen. Das Regional Government Coordinating Office beriet und überwachte den Länderrat in der amerikanischen Zone, damit die erste und wichtigste politische deutsche Instanz. Der Länderrat war ein Repräsentativorgan der vier beteiligten Landesregierungen von Bayern, Bremen, Hessen und Württemberg-Baden; er unterhielt neben einem Sekretariat zahlreiche Ausschüsse und Unterausschüsse. Zwischen ihnen und den zuständigen amerikanischen Beamten vermittelte das Regional Government Coordinating Office zahlreiche Besprechungen; es diente ganz grundsätzlich als Verbindungsstelle zwischen Besatzungsmacht und der deutschen Landesverwaltung. So wurden auch Beschlüsse und Vorschläge des Länderrats uber das RGCO OMGUS zugeleitet und der Länderrat anschließend von RGCO über die Entscheidungen der Militärregierungen unterrichtet.

Der erste Leiter des RGCO, James K. Pollock, übte sein Amt bis Juli 1946 aus. Seine Nachfolger wurden ab 15 . August 1946 Col. William W. Dawson, der gleichzeitig das Office of Military Government for Württemberg-Baden leitete und nach dessen plötzlichem Tod am 11. Februar 1947 (Lt.)Col. Charles D. Winning. 1948 wurde der Direktor Winning von einem Executive Officer, einem Senior Adviser, Anton Pabsch, einem Fachberater fur Wirtschaft, Carl R. Mahder, einem Fachberater fur Arbeit und Soziales, Heinz Guradze, und einem Fachberater für Überprüung der deutschen Gesetzgebung, Dewilda Naramore, und zwei Unterabteilungsleitern, für die Reports und Information Branch Myrtle Todd Moseley und für die Public Information Branch James G. Lyons, unterstützt. Auf deutscher Seite waren der Generalsekretär Erich Roßmann und sein Stellvertreter Hans Wutzlhofer die wichtigsten Partner.

Am 1.Juli 1948 wurde das Regional Government Coordinating Office durch General Order Nr. 24 aufgelöst und seine Aufgaben von OMGUS selbst je nach fachlicher Zuständigkeit übernommen.

\section{Bipartite Organization}

Parallel zu der deutschen bizonalen Verwaltung wurde seit August 1946 eine gemeinsame amerikanisch-britische Verwaltung (Bipartite Organization) geschaffen, um die deutschen Dienststellen zu überwachen. Oberste gemeinsame Dienststelle der beiden Besatzungsmächte war der Bipartite Board, welcher aus den beiden Stellvertretenden Militärgouverneuren bestand. Nach der Ernennung Clays zum Military Governor am 15. März 1947 behielt er sich die Mitgliedschaft im Bipartite Board vor; die britische Seite wurde weiterhin durch den Deputy Military Governor, Lt.Gen. Sir Brian $\mathrm{H}$. Robertson, vertreten. Dem Bipartite Board stand ein in Berlin geschaffenes Bipartite Secretariat in ähnlicher Weise zur Verfügung wie das Alliierte Sekretariat dem Alliierten Kontrollrat und dessen KoordinierungsausschuB. 
Sechs gemeinsame Fachausschüsse (Bipartite Committees) wurden in Berlin errichtet und umfaBten die Leiter der jeweiligen Fachabteilungen der amerikanischen und der britischen Militärregierung. Die Bezeichnung Committee wurde im November 1946 in Panel geändert, um eine Verwechslung mit den deutschen bizonalen Ausschüssen zu vermeiden. Eine entsprechende Anzahl von amerikanisch-britischen Bipartite Control Groups, die als Verbindungsstellen zu den Panels fungieren sollten, wurden am Sitz der entsprechenden deutschen Verwaltungsräte oder Verwaltungen errichtet.

Bis Februar 1947 wurden drei weitere gemeinsame Dienststellen der beiden Besatzungsmächte geschaffen: die Joint Export-Import Agency (JEIA), die Joint Foreign Exchange Agency (JFEA) und die Joint Vetting Party, der die Aufgabe übertragen wurde, geeignetes deutsches Personal für die bizonalen Dienststellen zu ermitteln und zu überprufen.

Im Mai 1947 wurden die deutschen bizonalen Wirtschaftsdienststellen neu organisiert: die Verwaltungsbehörden und Gemeinsamen Ausschüsse wurden abgeschafft und ihre Zuständigkeiten dem Wirtschaftsrat für das Vereinigte Wirtschaftsgebiet (kurz: Wirtschaftsrat) übertragen. Er bestand aus dem Wirtschaftsrat im engeren Sinne (Economic Council), dem Exekutivrat (Executive Committee) und den Direktoren (Executive Directors). Dieser neuen Struktur hatte die gemeinsame britisch-amerikanische Verwaltung verständlicherweise zu entsprechen. Durch die Übereinkunft der beiden angelsächsischen Mächte vom 29. Mai 1947 wandelten sich die Bipartite Control Groups zu Bipartite Groups im damals begründeten Bipartite Control Office, das je einen amerikanischen und britischen Leiter hatte. Durch General Order Nr. 47 vom 10. Juni 1947 wurde mitgeteilt, daß der frühere Assistant Deputy Military Governor und pensionierte Armeegeneral Clarence L. Adcock zum BICO US Chairman bestellt worden sei. Gegen Ende Juni 1947 wurde das Bipartite Control Office mit neuem Leben erfullt, indem BICO den Bipartite Board bei der täglichen Verwaltungsarbeit des deutschen Exekutivrates vertreten sollte.

Die Sekretariate des amerikanischen und britischen Vorsitzenden, die zusammen das Joint Secretariat bildeten, sollten die Verbindung zwischen der Militärregierung und dem Wirtschaftsrat sicherstellen. Im September 1947 wurden die Bipartite Panels als solche abgeschafft; ihre beiden Mitglieder behielten ihre Funktionen als Advisers des jeweiligen Militärgouverneurs. Die Arbeit der Panels wurde von den entsprechenden BICO Groups übernommen, die damals mit Ausnahme der Transport Group, die bis Oktober 1947 in Bielefeld verblieb, nach Frankfurt verlegt wurden. Seit dieser Zeit gab es keinen unmittelbaren Instanzenzug von OMGUS in Berlin zu den deutschen bizonalen Verwaltungseinrichtungen. Alle Weisungen wurden über den US Chairman des am 10. September 1947 in Frankfurt errichteten Bipartite Control Office mitgeteilt. Bei einer historischen Bewertung des Verhältnisses von BICO in Frankfurt und der OMGUS-Zentrale in Berlin läßt sich feststellen, daß die meisten Entscheidungen in Frankfurt fielen und praktisch nur die grundsätzlichen, politischen Fragen in Berlin entschieden wurden.

Im Januar 1948 führte man eine weitere Umorganisation der deutschen bizonalen und der britisch-amerikanischen Besatzungsdienststellen durch. Die Anzahl der Abgeordneten des Wirtschaftsrats wurde verdoppelt, seine Kompetenzen erweitert, der Exekutivrat durch einen Länderrat ersetzt und ein Verwaltungsrat aus den Direktoren der einzelnen Verwaltungen unter Leitung eines ressortlosen Oberdirektors geschaffen. Auf BICO bezogen hatte dies zur Folge, daB das amerikanische und britische Personal unterhalb der Ebene der beiden gemeinsamen Vorsitzenden, Adcock und Sir Gordon N. Macready, insoweit zusammengefaßt wurde, als die Leiter und Stellvertretenden 
Leiter der Unterabteilungen alternativ Briten oder Amerikaner waren. Das Prinzip der Doppelbesetzung hatte damit sein Ende gefunden. Die JFEA wurde damals von der JEIA übernommen und die so erweiterte Behörde nach Frankfurt verlegt.

Im August 1948 errichtete man gleichzeitig mit der Verwaltung für Arbeit zusätzlich eine Bipartite Manpower Group. Am 8. August 1949 wurde gemäB General Order Nr.33 vom 6. August 1949 Adcock als US Chairman, BICO, von seinem bisherigen Stellvertreter, Robert K. Phelps, abgelöst.

BICO war sachlich auch am European Recovery Program beteiligt; das ERP-Sekretariat stellte die Verbindung mit der Organisation für europäische wirtschaftliche Zusammenarbeit (Organization of European Economic Cooperation) in Paris und der European Cooperation Administration in den Vereinigten Staaten dar. Bizonale Interessen wurden von den Vertretern von BICO wahrgenommen.

Am 21.September 1949 wurde das Bipartite Control Office offiziell aufgelöst, wenngleich einige seiner Einrichtungen (wie z. B. die gemeinsame Coal Control Group) noch eine gewisse Zeit fortbestanden.

Da BICO nicht zur amerikanischen Besatzungsverwaltung im engeren Sinne gehörte, kann sich die Darstellung der personellen Entwicklung des Sekretariats und der Bipartite (Control) Groups auf folgenden Abriß für die amerikanische Seite beschränken.

Ubersicht 47: Organisation und Personal von Bipartite Control Office, 1947/1949

September 1947

Februar 1948

Juni 1948

April 1949

September 1947

Februar 1948

Juni 1948

April 1949
Bipartite Secretariat

US Secretary

\author{
Walter E. Mather \\ Walter E. Mather \\ Walter E. Mather \\ Walter E. Mather
}

Economics / Commerce and Industry Group

A. S. Barrows, US Member

A. S. Barrows, US Member

L. E. Spencer, Chief

L. E. Spencer, Chief
September 1947

Februar 1948

Juni 1948

April 1949

September 1947

Februar 1948

Juni 1948

April 1949

September 1947

Februar 1948

Juni 1948

April 1949

September 1947

Februar 1948
Food and Agriculture / Food, Agriculture and Foresty Group

J. C. Lynn, US Member

J. C. Lynn, US Member

Stanley Andrews, Chief

Stanley Andrews, Chief

Transport Group

Col. A. H. Schroeder, US Member

Col. A. H. Schroeder, US Member

C. P. Gross, Deputy Chief

W. A. Fagan, Deputy Chief

Communications Group

W. L. Slattery, US Member

W. L. Slattery, US Member

C. P. Rapp, Deputy Chief

W. J. Campbell, Deputy Chief

Finance Group

Lt.Col. Emery D. Stoker, US Member

Lt.Col. Emery D. Stoker, US Member 
Juni 1948

April 1949

April 1949

September 1947

Februar 1948

Juni 1948

April 1949

Juni 1948

April 1949

Juni 1948

April 1949

April 1949

April 1949

April 1949
Howard P. Jones, Deputy Chief

Howard P. Jones. Deputy Chief

Manpower Group

S. G. Wynn, Deputy Chief

Civil Service Group

Ellsworth D. Wolfsperger, US Member

Ellsworth D. Wolfsperger, US Member

R. C. Simonson, Chief

Ellsworth D. Wolfsperger. Chief

Decartelization Commission

Richard Bronson, US Member

Richard Bronson, Chief

Legal Group

Richard D. Kearney, Chief

Richard D. Kearney, Chief

US Customs Group

J. Miles, Chief

Central Brank Group

Henry C. Conrad, Chief

Combined Coal Control Group

W. J. German, US Chairman

Im Zusammenhang mit der Commerce and Industry Group, BICO, ist zu erwähnen, daB deren OMSTEG Branch die Aufsicht über die deutsche Staatliche ErfassungsGesellschaft für öffentliches Gut führte, welche damit beauftragt war, amerikanisches Heeres- und Beutegut zur Versorgung der deutschen Wirtschaft und Bevölkerung zu erfassen, zu übernehmen und zu verwerten.?

\section{Joint Export-Import Agency / Joint Foreign Exchange Agency ${ }^{8}$}

Am 1.Januar 1947 wurden die Joint Export-Import Agency und die Joint Foreign Exchange Agency als Zwei-Mächte-Einrichtungen zur Förderung des deutschen Außenhandels und zur Beaufsichtigung deutscher Devisentransaktionen einschließlich der Verwendung des als Startkapital gewährten amerikanisch-britischen Zuschusses geschaffen. Durch General Order Nr. 8 vom 27. Januar 1947 wurde Roy J. Bullock als amerikanisches Mitglied der JEIA unter Beibehaltung seiner bisherigen Funktionen bestellt. Die JFEA war gleichsam die Abrechnungsstelle der JEIA und blieb trotz der grundsätzlich beschlossenen Vereinigung mit JEIA angeblich aus praktischen Gründen bis zum Ende der OMGUS-Periode als eigene Dienststelle bestehen.

Aufsichtsorgan für die JElA war ein Board of Directors, der sich aus den Finanz- und Wirtschaftsberatern beider Militärregierungen sowie einem amerikanischen Generaldirektor und dessen britischen Stellvertreter zusammensetzte. Der Board, der Weisungen von BICO unterworfen war, tagte im Grundsatz monatlich. Im Sommer 1948 wurde JEIA, die ihren Sitz in Frankfurt-Höchst hatte, von dem Amerikaner William

\footnotetext{
${ }^{7}$ Vgl. J. Henke: Economic Reconstruction, S. 474.

8 Vgl. Das Bundesarchiv und seine Bestände, S. 407
} 
John Logan als Generaldirektor geleitet. Sieht man von der Dienststellenverwaltung ab, so stellte die Foreign Trade Division unter dem amerikanischen Direktor George J. Santry den Kern von JEIA dar, hinzu kam ein Office of the Comptroller unter Leitung des Amerikaners Moore C. McIntosh. Durch die Zusammenlegung der JEIA und des Office du Commerce Éxtérieur für die französische Zone am 18. Oktober 1948 entstand JEIA als Drei-Mächte-Behörde. Der Name blieb bestehen, doch erweiterte sich die Organisation erheblich. Im Frühjahr 1949 bestanden neben der Dienststellenverwaltung drei Offices: das Office of the Comptroller, das weiterhin von McIntosh geleitet wurde, das Office of Foreign Trade unter Leitung von C. E. Bingham und das Office of the Director of Operations unter M.S. Verner. Hinzu traten Zweigstellen (Branch Offices) in Berlin, Bremen, Düsseldorf, Hamburg, Hannover, Kiel, München, Stuttgart, Wiesbaden und Baden-Baden. Auch nach Übergang von OMGUS zu HICOG bestand JEIA als Behörde - allerdings mit verringerten Kompetenzen noch einige Zeit fort.

\section{Allied Control Authority}

Die grundsätzliche Struktur der Alliierten Kontrollbehörde ist bereits hinreichend beschrieben worden (OMGUS, A. 6). Es reicht daher aus, einen Überblick über die Untergliederungen der Direktorate zu geben. Dies soll dadurch geschehen, daB die Bezeichnungen der Ausschüsse nach dem Stand vom März 1946 und Februar 1948 in den einzelnen Fachbereichen miteinander verglichen werden.

Ubersicht 48: Direktorate und Ausschusse der Alliierten Kontrollbehörde, Mărz 1946

\section{Military (Amy) Directomte}

\section{Naval Directorate}

- Demilitarization Committee

- Steering Committee

Air Directorate

- Committee on Aviation

- Committee on Meteorology

- Air Intelligence Committee

Transport Directorate

- Rail Committee

- Highway and Highway Transport Committee

- Inland Waterways Committee

- Ports Committee

- Coastal Shipping Committee

Political Directorate

- Establishment of Interim Office for German Affairs Committee

\section{Economics Directorate}

- Food and Agriculture Committee

- Trade and Commerce Committee

- Industry Committee

- Level of Industry Committee

- Fuel Committee

- Central German Administrative Departments Committee

- Liquidation of German War Potential Committee

- I. G. Farben Committee 
Finance Directorate

- Cost of Transportation Intemees Committee

- Decentralization of German Public Finance Committee

- Valuation of Reparations Committee

- Costs of Occupation Committee

- Banking Committee

- Insurance Committce

- Property Control Committee - Provincial Loans Committee

Repardions, Deliveries, and Restitution Directornte

- Restitution Procedures Committee

- Reparation Procedures Committee

Lezel Directorate

- Patent Committee

- Control Council Gazette Committee

- Committee on Reopening of German Courts

- Working Party on Juvenile Delinquents

- Working Party on Criminal Code

- Working Party on Repeal of Nazi Laws

- Working Party on Revision of the Marriage Law

- Working Party on Hereditary Farm Laws

Prisoners of War and Displaced Persons Directorate

- Central Tracing Policy Board

- Combined Repatriation Executive

Manpower Directorate

- Labor Laws, Trade Unions, and Labor Relations Committee

- Labor Standards and Wages Committee

- Labor Supply and Allocation of Manpower Committee

- Committee on Social Insurance including Unemployment Relief

- Housing Problems Committee

\section{Internal Afiairs and Communications Directorate}

- Allied Posts and Communications Committee

- Allied Education Committee

- Health Committee

- Religious Affairs Committee

- Public Safety Committee

- Civil Administration Committee

- Intelligence Committee

- Nutrition Committee

- Public Welfare Committee

\section{German External Property Commission}

- Committee for External Assets Statistics Pooling

Im Februar 1948 ergab sich dagegen folgendes Bild: An die Stelle der drei militärischen Directorates (Military/Army/Interarmy, Naval und Air) war ein Combined Services Directorate getreten, das im Grundsatz über die Kompetenzen der drei genannten Direktorate verfügte. Sieht man einmal von der Berlin Kommandatura ab, so blieb die Zahl von neun „zivilen“ Direktoraten konstant; hinzu kam die German External Property Commission. Die neun Direktorate gliederten sich im Februar 1948 wie folgt. 
Übersicht 49: Direktorate und Ausschüsse der Alliierten Kontrollbehörde, Februar 1948

Transport Directorate (Col. Hans W. Holmer)

- Railways Committee

- Highways Committee

- Coastal Shipping and Ports Committee

- Inland Waterways Committee

- Movements Working Party Committee

Political Directorate (Warren M. Chase)

- Information Committee

Economics Directorate (Lawrence Wilkinson)

- Decartelization Working Party

- Permanent Reparations Secretariat

- Industry Committee

- Fuel Committee

- Food and Agriculture Committee

- Committee for Liquidation of German War Potential

- Trade and Commerce Committee

- I.G. Farben Control Committee

- Central German Administrative Departments (Economic) Committee (Vacant)

Finance Directorate (Theodore H. Ball)

- Banking Committee

- Committee for Balancing of Foreign Accounts

- Taxation Committee

- Price Policy Committee

- Insurance Committee

- Committee for Financing Reparations Costs

- Committee on Financing Occupation Costs

- Public Finance Committee

- Currency Printing Committee

- Financial Regulations Committee

Reparations, Deliveries, and Restitution Directorate (Col. John H. Allen)

- Restitution Procedures Committee

- Reparations Procedure and Valuations Committee

- Reciprocal Deliveries Committee

Legal Directorate (Alvin J. Rockwell)

- Legislative Drafting Committee

- Committee on Reform of German Law

- Committee for the Revision of the Criminal Law

- Industrial Property Committee

- Crowcass, Permanent Committee

Prisoners of War and Displaced Persons Directorate (Lt.Col. Harry S. Messec)

- Central Tracing Policy Board

- Combined Repatriation Executive

Manpower Directorate (Leo R. Werts)

- Labor Supply Committee

- Social Insurance Committee

- Trade Union and Labor Law Committee

- Wages and Labor Standards Committee

- Housing Committee 
Internal Affairs and Communications Directorate (George B. McKibbin)

- Civil Administration Committee

- Intelligence Committee

- Public Safety Committee

- Allied Education Committee

- Allied Welfare Committee

- Allied Health Committee

- Allied Communications and Posts Committee

- Allied Religious Affairs Committee

German Extend Property Commission (E. J. Cassody) 


\section{Quellenbeschreibung}

\section{USGCC und Adjutant General}

Die unter USGCC 1944/45 und 17/8309 zusammengefaBten Unterlagen dokumentieren die Geschichte der Besetzung Deutschlands einschließlich Berlins durch amerikanische Truppen und der Errichtung der Militärregierung von Mitte 1944 bis zur Begründung von OMGUS im Oktober 1945 (mit Nachakten bis Ende 1946).

Die Kompetenz des Adjutant General auf dem Gebiet der Aktenfuhrung erklärt, warum in diesem Teilbestand der Gesamtüberlieferung des Office of Military Government for Germany die Akten der Spitzengliederung, also vor allem des Militärgouverneurs selbst, dessen Vertreters, des Chef des Stabes, des Generalsekretärs u. a. zusammengefaBt sind. Die offenen Akten einschlieBlich der Verschlußsachen bis zum Geheimhaltungsgrad Secret wurden nach einem Aktenplan des War Department organisiert, dem das Prinzip der Dezimalklassifizierung zugrunde gelegt ist. Insgesamt sind 487 Boxes überliefert, die sich - jahrgangsweise organisiert - wie folgt gliedern:

AG 1945/46 Boxes $1-112$

AG 1947 Boxes $1-194$

AG 1948 Boxes 1 - 188

AG 1949 Boxes $1-93$

Angesichts ihrer besonderen historischen Bedeutung soll die Überlieferungsschicht der Jahre 1945/46 aus dem Teilbestand des Adjutant General im folgenden detailliert beschrieben werden, wobei die Darstellung dem Aktenplan folgt.

Unter 0.1 sind $u$. a. Akten uber die ersten freien Wahlen in der amerikanischen, der britischen und der sowjetischen Zone sowie in Berlin zusammengefaBt, auBerdem Fragen der politischen Struktur der amerikanischen Besatzungsszone und des Aufbaus der Parteien dokumentiert, so auch die friben Auseinandersetzungen um den CSU-Politiker Josef Muller. Unter 0.3 finden sich Kirchensachen, insbesondere des Protestantismus, unter 0.5 Angelegenheiten der Kriegsverbrecherprozesse. Die Akten unter 0.7 behandeln Probleme des Wiederaufbaus der Medien, vor allem der Zeitungen und sonstigen Presseorgane, dokumentieren aber auch die Pressekonferenzen, die seitens der amerikanischen Militărregierung selbst gegeben wurden. Unter 0.8 und 0.9 ist der Wiederaufbau des Unterrichtswesens und der wissenschaftlichen Forschung belegt.

Das Aktenzeichen 0.4 war für Unterlagen über grundlegende Fragen der Wirtschafts-, Industrie- und Entflechtungspolitik - u. a. auf dem Banksektor - vorgesehen; die Akten enthalten aber auch Informationen uber Fragen der Arbeitsverwaltung, der Gewerkschaften und der Behandlung des Vermögens der Deutschen Arbeitsfront. Unter 0.5 sind Akten zu Fürsorgemaßnahmen zugunsten der deutschen Bevölkerung überliefert, unter 0.7 Unterlagen zu Problemen der Kunst, der Archive, der Museen und anderer kultureller Einrichtungen.

Die unter dem Aktenzeichen 0.10 erfaBte Dokumentation bietet Informationen über die Landesverfassungen, den Neuaufbau der Justizverwaltung, aber auch der Landesverwaltungen im allgemeinen, die Gesetzgebung der Besatzungsmacht und der deutschen Länder sowie über Verwaltungsvorschriften unterschiedlicher Sachbereiche. Unter 0.14 wird der Aufbau der Militärregierung, aber auch der deutschen Verwaltungen, insbesondere auf der Ministerialebene, des Länderrats und seiner Ausschüsse do- 
kumentiert. Hervorzuheben ist auch hier die Berichterstattung sowohl der Militärregierung als auch nachgeordneter amerikanischer und deutscher Stellen an die Militärregierung. Inhaltlich sind Fragen der Fraternisierung, der Entnazifizierung, vor allem im Bereich der Hochschulen, des Unterrichtswesens, der Bahn und der Post sowie Angelegenheiten der Verschleppten, der Flüchtlinge und Vertriebenen vieler Nationalitäten einschließlich der Juden dokumentiert. Unter 0.15 werden Fragen sowohl der deutschen wie der amerikanischen Gerichte behandelt.

Das Aktenzeichen 0.32 war Kontrollbesuchen amerikanischer Dienststellen in Deutschland vorbehalten und enthält daher zum Teil grundlegende Informationen zur amerikanischen Besatzungspolitik. Die Tätigkeit der amerikanischen Ministerien (Departments) ist unter dem Aktenzeichen 0.40 belegt, während unter 0.50 statistische Unterlagen, unter 0.62 Aktivitäten auf dem Gebiet der Fotografie und des Films, unter 0.72 Patentangelegenheiten überliefert sind.

Unter 0.80 finden sich Informationen über soziale Hilfsmaßnahmen, insbesondere der UNRRA, des Roten Kreuzes und amerikanischer Einrichtungen, zugunsten der deutschen Bevölkerung ebenso Informationen über einen „Betriebsrat“ (OCEA) bei OMGUS selbst.

Die Akten unter 0.91 enthalten Informationen über die Gründung der amerikanischen Besatzungszone. Weitere Unterlagen befinden sich hier über Norwegen, Polen und Ungarn, aber auch über den Wiederaufbau Europas im allgemeinen, den Kontrollrat, den Rat der Außenminister, die deutsche Wirtschaft, Finanz- und Handelsfragen, die öffentliche Meinung, das Wohnungs- und Gesundheitswesen, Verkehrsangelegenheiten, die Internationalisierung der Ruhr und den Status des Saargebiets. Die Akten unter 100 beschäftigen sich mit Währungsfragen einschließlich der Behandlung von Auslandsvermögen in Deutschland. Unter 121 finden sich u. a. Haushaltsvoranschläge von OMGUS, unter $130 \mathrm{ff}$. Informationen uber Probleme des Rechnungswesens sowie schließlich unter 140 Fragen der Verwaltung von öffentlichem und privatem Eigentum. Unter dem Aktenzeichen 150 werden Forderungen vor allem ausländischer Staaten gegen die amerikanische Besatzungsmacht dokumentiert.

Unter 200 sind Akten über Probleme der Identifizierung von Personen, der Personalplanung, des internationalen Reiseverkehrs überliefert, unter 230 lassen sich Grundsätze über die Einstellung vor allem deutschen Personals bei OMGUS ermitteln. Besuche amerikanischer Geschäftsleute und Berichte über deutsche Wissenschaftler, Techniker und anderer Experten in amerikanischem Gewahrsam sind unter 231 erfaßt. Angelegenheiten der Lohnpolitik in Deutschland, auch für nichtdeutsche Arbeitnehmer, finden sich unter $240 \mathrm{ff}$.

Unter dem vagen Titel Incidents (Zwischenfälle) sind bei 250 so verschiedenartige Gegenstände wie Aktivitäten von Polen auf dem Schwarzen Markt, Angriffe von Deutschen auf Angehörige der Besatzungsmächte und ein Aufruhr jüdischer Internierter in einem DP-Lager, aber auch Auseinandersetzungen zwischen amerikanischen und sowjetischen Soldaten dokumentiert. Unter 254 finden sich Unterlagen über Internierungs-, Kriegsgefangenen- und andere Lager.

Das Aktenzeichen 291.1 war Problemen der amerikanischen farbigen Soldaten vorbehalten, unter 293 finden sich Vorgänge über die Benachrichtigung von Angehörigen deutscher Kriegsgefallener. Unter 311 sind Probleme der Verkehrs-, Post- und Nachrichtenpolitik zusammengefaßt.

Von herausragender politischer Bedeutung, insbesondere für Fragen der Außenpolitik, ist die Überlieferung unter dem Aktenzeichen: 312 Correspondence, Political Affairs. So finden sich hier Denkschriften u. a. des Political Adviser, Robert Murphy, an General Clay uber allgemeine Fragen der Deutschland- und Europapolitik. 
Unter 313.5 finden sich Informationen über die Verfilmung von Akten deutscher Wirtschaftsunternehmen, unter 314.4 über Grundsatzfragen der Beschlagnahme, Benutzung und weiteren Behandlung deutscher Akten. Unter 314.7 wurden Fragen der Militärgeschichte einschließlich der amerikanischen Besatzungsverwaltung in Deutschland behandelt; schließlich finden sich unter 314.81 Kriegstagebücher der Vorläuferorganisation von OMGUS, der USGCC, aber auch Korrespondenz über die Tagebücher von Eva Braun mit einer englischen Übersetzung des Abschnitts von Februar bis Mai 1935.

Unter dem Aktenzeichen 319.1 Reports sind vorwiegend Berichte nachgeordneter Stellen der Militärregierung, aber auch zusammenfassende Darstellungen über internationale Konferenzen, z. B. die Pariser Reparationskonferenz Ende 1945, enthalten. Diese Überlieferung ist vor allem geeignet, fehlende Berichte auf regionaler Ebene zu ersetzen.

Unter dem Aktenzeichen 320 sind Aufbau und Organisation der Militärregierung auch aus der Zeit vor der Gründung von OMGUS belegt. Bei den Aktenzeichen 321 und 322 liegt der Schwerpunkt auf der Beschreibung von Zuständigkeiten nahezu aller Verwaltungseinheiten von OMGUS nicht nur auf der Ebene der Divisions, sondern auch von kleineren nachgeordneten Stellen wie z.B. dem Ministerial Collecting Center einerseits und allierter Einrichtungen wie dem Kontrollrat und der Kommandatura in Berlin andererseits.

Unter 333 sind Inspektionsberichte bis hinunter auf die Ebene der Kreise zusammengefaBt; auch hier finden landes- oder regionalgeschichtlich interessierte Forscher ebenso reiches Material wie Wissenschaftler, die sich für bestimmte verwaltungsgeschichtliche Probleme oder Personen und deren Tätigkeit in der Zeit des NS-Regimes interessieren.

Unter 334 befinden sich ebenfalls verwaltungsgeschichtlich relevante Sachakten uber einzelne Besatzungsdienststellen oder internationale Einrichtungen wie die European Economic Commission (EEC) oder die Alliierte Kontrollbehörde.

Zum Aktenzeichen 337 sind geschäftstechnische Serien, insbesondere Protokolle von Besprechungen innerhalb der amerikanischen Militärregierung, zusammengefaBt, so auch eine Niederschrift über die Potsdamer Konferenz, die wöchentlichen Staff Conferences von USGCC bzw. OMGUS, dazu Treffen der amerikanischen und britischen Stellvertretenden Militärgouverneure.

Unter 350 sind erneut Akten über Fragen des Unterrrichts und des Wiederaufbaus der Schulverwaltungen in Deutschland einschlieBlich der Reeducation uberliefert. Die Unterakten zu 350 enthalten bemerkenswertes Material: So finden sich unter 350.05 Unterlagen über Konflikte mit sowjetischen Stellen, unter 350.09 geheimdienstliche Berichte und ähnliche Unterlagen, unter 350.5 Informationen über die Behandlung deutscher Forscher und Techniker.

Beim Aktenzeichen 353 sind soziale Maßnahmen zugunsten der Angehörigen der Besatzungsverwaltung und der deutschen Bevölkerung zusammengefaBt. Unter 360 werden Fragen des Luftverkehrs, unter 370.2 die Aktionen „Goldcup“ und „Paperclip“ sowie andere „Operations“, unter 371.1 die amerikanische Information Control Policy dokumentiert.

Unter dem Aktenzeichen 383 finden sich politische Grundsatzerörterungen über die Entnazifizierung, über die Behandlung von Kriegsgefangenen und Spionen, Verschleppten, Internierten u. a. m. Die Akten unter 386 dokumentieren Fragen des Eigentumsrechts, u. a. der ausländischen Vermögen in Deutschland und deutscher Vermögen im Ausland. Während unter 387 Ereignisse gegen Kriegsende - so die Kapitulation der deutschen Truppen in Norditalien - belegt sind, finden sich bei 388 
Akten zu Fragen der Entwaffnung und Auflösung der deutschen Streitkräfte ebenso wie über die industrielle „Abrüstung“ Deutschlands.

Unter $\mathbf{4 0 0}$ wurden Probleme der Versorgung der amerikanischen Besatzungstruppen in Deutschland, unter 403 Fragen der Landwirtschaft, unter 410 und 411 Probleme der Wirtschaftspolititik, insbesondere der Produktion von bestimmten Rohstoffen und Fertigprodukten behandelt. Die Versorgung mit Lebensmitteln und Arzneien ist unter dem Aktenzeichen 430 bzw. 440 ff. dokumentiert. Das Aktenzeichen 453 weist Unterlagen über die Ausristung der Eisenbahnen insbesondere auch im Verhältnis zu anderen Zonen aus. Unter 461 findet sich Material uber die Beschlagnahme nationalsozia-

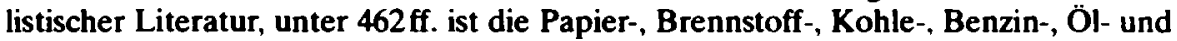
Kunstdüngerzuteilung behandelt.

Die Akten unter $\mathbf{5 0 0}$ ff. dokumentieren Fragen der Wirtschaftspolitik, die unter $600 \mathrm{ff}$. Angelegenheiten des Bauwesens, der Reparationen sowie der Rückerstattung von Grundstücken. Unter $700 \mathrm{ff}$. finden sich Akten über die Gesundheits-, unter $800 \mathrm{ff}$. über die Wasserstraßen- und Schiffahrtspolitik.

Nach diesem - hier für die Jahre 1945/46 kursorisch vorgestellten - Aktenplan sind auch die Unterlagen für die folgenden Jahrgänge 1947, 1948 und 1949 organisiert. Dabei stehen fur das Jahr 1947 Fragen der Ermittlung und Bestrafung von Kriegsverbrechern, der Pressepolitik, der Entflechtung der Wirtschaft, der Verfassungsgesetzgebung in deutschen Ländern, der Rechtsetzung durch die Militärregierung, der Entnazifizierung, der Flüchtlinge und Vertriebenen aller Nationen, des Wiederaufbaus des wirtschaftlichen und kirchlichen Lebens, Hilfsaktionen internationaler wie amerikanischer Einrichtungen, der Aufbau der Bizone und damit verbundene wirtschafts- und landwirtschaftspolitische Fragen, die außenpolitische Vertretung Deutschlands, der Marshallplan, die Londoner Außenministerkonferenz und andere Fragen der Außenpolitik im Vordergrund.

Der Jahrgang 1948 wird eroffnet mit einem Band uber eine Demonstration gegen die Wirtschaftspolitik Ludwig Erhards am 28. Oktober 1948 in Stuttgart. Die politische Situation unter besonderer Berücksichtigung der Erkrankung des SPD-Vorsitzenden Kurt Schumacher, die Berliner Blockade, die ersten Messen in Leipzig und Hannover, die Feiern zur 100jährigen Wiederkehr der Revolution von 1948, geheimdienstliche Aktivitäten, die Entnazifizierung, Displaced Persons, Reparationen und Restitutionen werden vorrangig behandelt.

Die Überlieferung für das Jahr 1949 ist schon vom Umfang her bedeutend geringer. Hervorzuheben sind insbesondere Unterlagen über die Auswirkung der Währungsreform, die Fortführung der Entflechtung der Wirtschaft, die Arbeits- und Sozialpolitik, die deutsche öffentliche Meinung, den Marshallplan, die Beendigung der Berliner Blockade und die Verhandlungen der drei Mächte über die politische Struktur der späteren Bundesrepublik Deutschland, dabei insbesondere zum Übergang der Verantwortung für Deutschland vom War Department auf das State Department, die Treffen der drei Militärgouverneure in den Monaten bis zur Gründung der Bundesrepublik Deutschland, die Außenministerkonferenzen, die Reorganisation an der Ruhr und die Entsendung deutscher Wissenschaftler in die Vereinigten Staaten von Amerika.

Die Überlieferung des Adjutant General wird durch 172 Boxes mit Akten der Geheimhaltungsstufe „Top Secret (TS)“ ergänzt, bei denen fast jedem Band ein Rotulus beigegeben ist, der einen Überblick über alle Schriftstücke bzw. Vorgänge bietet. Dieses Material ist für die politische Geschichte im Deutschland der Nachkriegszeit von besonderer Bedeutung. Es dokumentiert nicht nur die Entwicklung der amerikanischen und alliierten Deutschlandpolitik, sondern auch Vorgänge im Deutschland wäh- 
rend der NS-Zeit. Im Unterschied zu den jahrgangsweise organisierten offenen Akten erstrecken sich die VerschluBsachen zeitlich von 1944 bis 1949 und enthalten in erheblichem Umfang Informationen - oft in Form von Geheimdienstberichten - auch über die Nachbarstaaten Deutschlands. Der Überlieferungsform nach sind Unterlagen über Kontrollratsgesetze (mit Durchführungsvorschriften für die amerikanische Zone), Rechtsverordnungen (Regulations), Direktiven (Directives) und Allgemeine Verfügungen (General Orders) hervorzuheben.

\section{Chief of Staff}

In der Bedeutung ähnelt dieser Aktenbestand den Unterlagen des Adjutant General, vom Umfang her handelt es sich allerdings allenfalls um ein Zehntel des AG-Materials. Eine jahrgangsweise Gliederung liegt nicht vor, vielmehr unterscheiden sich die Akten nach einer Gruppe mit der Bezeichnung COS (1 - 7) anstelle einer ShipmentNummer und einigen kleineren Überlieferungsteilen, die im Zusammenhang mit anderen Akten nach Amerika verbracht wurden und daher eine Shipment-Nummer aufweisen $(2 / 135,3 / 176-3,3 / 177,17 / 162,17 / 8185)$.

COS 1 enthält Unterlagen über die Verbindungswege nach Berlin, insbesondere auf der Schiene, die Verwaltung der Militärregierung in Bayern, Korrespondenz zwischen OMGUS und der sowjetischen Militäradministration im zweiten Quartal 1948, das erste Treffen der Alliierten Hohen Kommissare vom 19. September 1949 sowie persönliche Unterlagen von General Gailey und Mrs. Marjorie Clay. Unter COS 2 finden sich u. a. einige Unterlagen zur Moskauer Außenminister-Konferenz im Jahre 1947. COS 3 umfaßt ebenfalls Korrespondenz mit der sowjetischen Militäradministration (April 1948 bis Mărz 1949) über Fragen der Währungsreform, Luftzwischenfălle u. a. m.

COS 4 dokumentiert Planungen der amerikanischen Regierung uber die Besetzung Deutschlands aus dem Spätsommer 1944, Unterlagen uber den Luftverkehr in Deutschland, über Kriegsgefangene und Displaced Persons, über die Begründung von Jugendclubs in Franken sowie zwei Wochenberichte des Bayerischen Staatsministeriums der Finanzen über Schmuggelaktivitäten.

COS 5 enthält Unterlagen zum hessischen Gesetz über Zwangsarbeit, über die Versorgung der deutschen Angeklagten in Nürnberg, die wirtschaftliche Vereinigung der britischen und der amerikanischen Zone, jugendpolitische Maßnahmen, einen Bericht über eine Reise an die Saar, über Clays Besuch an der Ruhr, RückerstattungsmaBnahmen zugunsten von Ungam, Kohlelieferungen, Angelegenheiten der Displaced Persons, über die Lage der Zivilinternierten, Besuche von KongreBabgeordneten, die Situation der Schiffahrt in Bremerhaven, die Ernährungslage in Mannheim, Besuche in der sowjetischen Besatzungszone u. a. m. COS 6 umfaBt sieben Bände mit Vorlagen an General Clay und interne Korrespondenz der Militärregierung von Januar bis Dezember 1947.

COS 7 behandelt Wahlen, Personalangelegenheiten, Besuche prominenter Amerikaner, Auseinandersetzungen innerhalb der sowjetischen Besatzungsmacht, das Außenministertreffen 1947 in London, Fragen der Ruhrkohle, einen antisemitischen Brief in der Süddeutschen Zeitung, den Wahlkampf der KPD, Behandlung von deutschem beschlagnahmtem Registraturgut, insbesondere von Unterlagen der Reichskulturkammer, einen Rechenschaftsbericht des Gesetzgebungsreferates der OMGUS-Abteilung für Rechtsfragen (1947 - 1949) und Unterlagen über Gespräche mit deutschen Wirtschafts- und Gewerkschaftsführern.

Die Box 135-1 im Shipment 2 erstreckt sich zeitlich von September 1946 bis März 1949 
und dokumentiert Fragen der Kriegsverbrecherprozesse und der Rückkehr von Flüchtlingen aus der sowjetisch besetzten Zone, Probleme der deutschen Wissenschaftspolitik von 1936 bis 1945 (mit Korrespondenz zwischen Clay und Otto Hahn über die Zukunft der Kaiser-Wilhelm-Gesellschaft), Fragen des Bankwesens, des Streiks an der Ruhr und des kommunistischen Einflusses, der Vertriebenen und der Ruckkehr von Displaced Persons in ihre Heimat (auch Schriftwechsel mit der Sowjetischen Milităradministration), Probleme der Freigabe deutscher Filme aus der Zeit vor 1945, der Entwicklung der Gewerkschaften, der Wahlen in Berlin, des Außenhandels, der Bezahlung der amerikanischen Besatzungssoldaten, Angelegenheiten mehrerer Abteilungen und Einheiten von EUCOM, Fragen der Beschäftigung von Amerikanern, die früher Staatsangehörige von Feindstaaten waren, die Zusammenarbeit mit der Sowjetischen Militäradministration bei Grenzzwischenfällen und Auseinandersetzungen in Berlin, Fragen des Reiseverkehrs und der Reorganisation EUCOM, Untersuchungen uber das Ende von Hitler und Bormann, uber Luftverkehrsfragen sowie den Malmedy-ProzeB.

Box 2/135-2 enthält Berichte über politische Aktivitäten der Deutschen auf Landkreisebene, über französisch-bayerische Kontakte, über die Wirtschaftslage, insbesondere auf dem Ernährungssektor in der sowjetischen Besatzungszone, über das Außenministertreffen in Moskau 1947, ein belgisch-amerikanisches Übereinkommen über die Rückkehr von belgischen Kriegsgefangenen, über Displaced Persons unter besonderer Berücksichtigung von Ukrainern, über die Frage eines Ultraviolett-Quarzmikroskops der Firma Zeiss, den Haushalt von OMGUS für das Jahr 1948 sowie Korrespondenz über die Luftbrücke (August bis Oktober 1948) und die Berliner Blockade im allgemeinen.

Box 2/135-3 umfaBt in erheblichem Umfang Denkschriften, die General Clay vorgelegt und von ihm teilweise kommentiert wurden, dokumentiert Fragen der Dekartellisierung, der Berliner Blockade und Luftbrucke, Reparationen und enthält sieben Bände über den Malmedy-Prozeß.

In 3/176-3 sind Unterlagen des Chief of Staff enthalten. Die Unterlagen betreffen die Rückführung sowjetischer Staatsangehöriger nach dem Abkommen von Jalta, die in Landsberg einsitzenden Kriegsverbrecher, Fragen des Patentwesens, der Finanz- und Wirtschaftspolitik, der britisch-amerikanischen Zusammenarbeit in Wirtschaftsfragen, insbesondere zur Zeit der Berliner Blockade, aber auch die allgemeine Wirtschaftspolitik von 1945 bis 1949; hinzu kommt die Korrespondenz zwischen Clay und Robertson über den Status des Landes Bremen aus dem Oktober 1946.

Die Box 3/177-1 dokumentiert Wahlen in mehreren Zonen und in Berlin, Fragen der sowjetischen Besatzungspolitik in Deutschland und Österreich unter besonderer Berücksichtigung der Beziehungen von OMGUS zur Sowjetischen Militäradministration in den Jahren 1947 bis 1949, die französische Obstruktion gegen die Errichtung zentraler deutscher Dienststellen und andere Fragen aus dem Bereich der inneren Verwaltung (u. a. Korrespondenz Clays mit Washington, Fragen der Entnazifizierung, September 1945 bis Mai 1947), die amerikanisch-französischen Beziehungen (Juli 1945 bis Dezember 1947) unter besonderer Berücksichtigung der Saarfrage sowie Angelegenheiten des Marshallplanes, der Verfassungen der Länder der amerikanischen Besatzungszone und der Entnazifizierung.

Der Schwerpunkt der Überlieferung in Box 3/177-2 liegt in der Darstellung der Entwicklung bizonaler Einrichtungen sowie der britisch-französischen Politik gegenüber der deutschen Wirtschaft und der Frage einer französischen Beteiligung am Aufbau der Bizone. AuBerdem behandelt werden die Steuerreform, die Bodenreform, die Akkreditierung ausländischer Militärmissionen, die Luftbrücke, die Arbeitskämpfe, 
die Währungsreform, der Internationale Militärgerichtshof in Nürnberg. Schwarzmarktaktivitäten, subversive Tätigkeit der Sowjets gegen die SPD in Berlin (August 1946) sowie Beziehungen zwischen OMGUS und den deutschen Ländern unter besonderer Berücksichtigung des Länderrats und der Vereinigung der drei westlichen Besatzungszonen.

In Box 3/177-3 finden sich Unterlagen über die Organisation der Militärregierung, die funktionalen Verbindungen zwischen OMGUS und USFET, Grenzzwischenfälle mit den sowjetischen Streitkräften, Angelegenheiten der Juden, Haushaltsfragen, Entnazifizierung. Verschleppungen und Erschießungen von früheren HJ-Führern in der sowjetischen Zone, den Hoover-Bericht über die deutsche Ernährungs- und Landwirtschaftspolitik, die Ruhrfrage, über die New Yorker Außenministerkonferenz (November bis Dezember 1946), gewerkschaftliche Aktivitäten (Juni 1946 bis Februar 1949), die Vorbereitung des Zusammenschlusses der drei westlichen Besatzungszonen und andere Fragen gesamtdeutscher Politik. Das Programm der Militärregierung im August 1945, Berichte über die Entwicklung des Unterrichtswesens, über die Potsdamer Konferenz, Vier-Mächte-Verhandlungen über die deutsche Industrie (1946) und die Moskauer Außenministerkonferenz im Frühjahr 1947 sind ebenfalls beigefügt.

Die Box 17/162-1 dokumentiert die Fragen der Innenpolitik unter besonderer Berücksichtigung der Displaced Persons, der Vertriebenen und Flüchtlinge, Zusammenstöße mit sowjetischen Soldaten, die Organisation des Länderrats und dessen Beziehung zum Regional Government Coordinating Office, Fragen der Entnazifizierung (1946), der bayerischen Monarchisten, der sowjetisch-amerikanischen Beziehungen (1946), Berlins und enthält Handakten von General Keating als amtierendem Stellvertretendem Militärgouverneur mit einem Bericht über die Niedersächsische Landespartei und den WAV-Vorsitzenden Loritz sowie einem biographischen Abriß uber den General.

Box 17/162-2 dokumentiert die personelle Ausstattung der Militărregierung Württemberg-Baden, die Errichtung der Bizone (1946), Fragen des Arbeitseinsatzes, die Neuorganisation der Bizone und die Berlinpolitik samt den Erfordernissen der Luftbrucke.

In Box 17/8185 liegen Unterlagen über die Zusammenarbeit der drei westalliierten Militärgouverneur vom August 1948 bis August 1949 vor. Sie betreffen inhaltlich vor allem die künftige politische Organisation Westdeutschlands und die Beziehungen der Besatzungsmächte zu den Ministerpräsidenten der Länder und zum Parlamentarischen Rat. Sechs Bände (21 - 26) belegen die Vorbereitung der Gründung der Bundesrepublik Deutschland umfassend. Weitere Akten betreffen das Besatzungsstatut, das Vertriebenenproblem, die Bildung des Tripartite Military Security Board im Herbst 1948, die Treffen der drei Militärgouverneure (Oktober 1948 bis September 1949) und wirtschaftspolitische Fragen.

\section{Allied Control Authority}

Die verhältnismäßig umfangreichen Akten des amerikanischen ACA-Sekretariats enthalten im wesentlichen Auszilge aus Sitzungsprotokollen, Vermerken und Denkschriften zum jeweiligen Beratungsgegenstand. Sie sind durch Übersichten und Indices in Form von Karteikarten erschlossen. Die Kontakte des Sekretariats zu den Direktoraten und Ausschüssen sind in chronologischer Folge gut belegt. Dies gilt auch für die Akten der Direktorate selbst, wobei innerhalb der Unterlagen eines jeden Direktorats die Chronologie das herrschende Ordnungsprinzip darstellt. Außerdem sind 
geschäftstechnische Serien der einzelnen Verwaltungseinheiten der ACA vorhanden.

Die ACA-Unterlagen sind in der Regel links oben auf einer jeden Seite durch Signaturen gekennzeichnet, aus denen die Provenienzstelle, die Art des Dokuments und der Zeitpunkt der Entstehung abzulesen sind.

\section{Bipartite Control Office}

Hervorzuheben sind vor allem Unterlagen über das European Recovery Program (ERP) und die Organization of European Economic Cooperation (OEEC) sowie der European Cooperation Administration (ECA) in den Vereinigten Staaten.

Es ist deutlich zu unterstreichen, daB nicht nur Akten der Economics Division, sondern auch andere Unterlagen aus den Bereichen Finanzen, Ernährung, Landwirtschaft, Verkehr und schließlich der Verwertung öffentlichen Guts für den Bereich des Außenwirtschaftsverkehrs und die Unterlagen der Joint Export-Import Agency (JEIA) sowie der Joint Foreign Exchange Agency (JFEA) in diesem Teilbestand enthalten sind.

\section{Regional Government Coordinating Office}

Die Aktenstruktur entspricht der Aktenführung des deutschen Pendants des RGCO, des Länderrats für das amerikanische Besatzungsgebiet. So finden sich Akten, insbesondere Protokolle von Länderratsausschüssen und Unterausschüssen mit Bemerkungen des Beobachters des RGCO (,highlight summeries as noted by RGCO observer“) für alle Verwaltungszweige.

Hinzu kommen chronologisch organisierte Durchschriften der Korrespondenz des Leiters des RGCO vom Juli 1946 bis Juni 1948 und Schriftwechsel mit dem Länderrat über die Zukunft der I. G. Farben Industrie. Hervorhebenswert ist ein Band über die Wirtschaftsplanung der Besatzungsmacht mit Unterlagen, die teilweise in das Jahr 1944 zurückgehen. Seit Mitte 1946 spiegeln sich in den Unterlagen von RGCO auch die Wirtschaftsentwicklung in der britischen Zone und selbstverständlich der Aufbau der Bizone. Bemerkenswert ist ferner die Korrespondenz über politische und verfassungsrechtliche Grundsatzfragen mit dem Deutschen Büro für Friedensfragen in Stuttgart.

Die Unterlagen über die Besprechungen der Besatzungsmacht mit den Ministerpräsidenten der amerikanischen Zone sind in ihrer inhaltlichen Aussage über entsprechende Materialien im Bestand „Länderrat des amerikanischen Besatzungsgebietes“ (Z 1) im Bundesarchiv hinaus wegen der internen amerikanischen Beurteilungen über die jeweilige politische Situation von Bedeutung. Im Aktenband über Kommunalwahlen, Wahlen zu den verfassunggebenden Landesversammlungen sowie über die Verfassungen und Parteiprogramme in den Ländern ist ein Bericht eines amerikanischen Offiziers über Reden des SPD-Vorsitzenden Schumacher ebenso hervorzuheben wie eine Besprechung mit dem Präsidenten der Deutschen Zentralverwaltung für Arbeit in der sowjetischen Zone, Brack, über eine angeblich neue antikommunistische Kampagne in der Presse der amerikanischen Besatzungszone.

Ein besonderer Schwerpunkt liegt bei der Frage der Entnazifizierung, der Kriegsverbrecherprozesse und anderer Rechtsfragen. Die Gründung des Instituts für Zeitgeschichte (als Institut zur Erforschung der nationalsozialistischen Zeit) in München ist 
in 11/35-3/7 belegt. Aus dem Bereich der Unterrichtsverwaltung sind Akten über die Wiedererrichtung der Reichsanstalt für Film und Bild in Wissenschaft und Unterricht und politische Wertungen über den bayerischen Staatsminister Alois Hundhammer von besonderer Bedeutung.

Von den umfangreichen Ausschußunterlagen sind die Materialien über Kampfstoffe und über die Stahlproduktion (auch in bezug auf die Röchling-Werke) von besonderer Bedeutung. Hervorzuheben sind ferner eine Besprechung des Länderratsausschusses für Kriegsgefangenenwesens mit der Sowjetischen Militäradministration in Berlin im November 1947 und ein Bericht über eine Reise zweier hessischer Minister nach Thüringen im Juni 1946.

Erwähnt werden sollte schließlich auch der Band 11/39-3/8, der u. a. Vorwürfe gegen den württemberg-badischen Ministerpräsidenten Maier wegen dessen Zustimmung zum Ermächtigungsgesetz im Jahre 1933 enthält. Die politisch wichtigste Dokumentation des Teilbestandes RGCO enthält der Band 11/42-3/1, in dem die privatdienstliche Korrespondenz General Clays mit den jeweiligen Leitern des RGCO uberliefert ist; darin finden sich Informationen uber die österreichischen Wahlen im Dezember 1945. die Ansichten Clays über die Bodenreform 1945/46, Maßnahmen zur Überwachung von Displaced Persons (Dezember 1945), Vorschläge des hessischen Ministerpräsidenten Geiler zur Errichtung von Zentralverwaltungen (Februar 1946), französische Bemühungen um Nord-Württemberg (April 1946), die Gründung des Landes Niedersachsen (April 1946), Bemerkungen über den Besuch des hessischen Finanzministers Matthes in Weimar (Juni 1946), die Vier-Zonen-Konferenz der Ministerpräsidenten in Müchen (Juni 1947), die Bemuhungen des Bremer Senats um die Rlickfuhrung von Archivalien aus der UdSSR (Dezember 1947) und ein Treffen des französischen Militärgouverneurs, Gen. Pierre Koenig, mit den Ministerpräsidenten der französischen Zone (Fruhjahr 1948).

\section{Fachämter und Fachabteilungen}

Da eine detaillierte Beschreibung der Teilbestände der Fachämter und Fachabteilungen zu Redundanzen führen wurde, beschrănkt sich die folgende Darstellung auf eine summarische Nennung der in Deutschland in Mikroficheform zugänglichen Überlieferungen.

\section{Control Office}

Das Control Office stellte gleichsam das Organisationsreferat der Militärregierung dar. In seinen umfangreich erhaltenen Unterlagen $(5 / 353-376 ; 11 / 44-50)$ finden sich Informationen über sämtliche Bereiche der Militärregierung. Dabei stehen Probleme der Dienststellenverwaltung und sog. Querschnittsaufgaben im Vordergrund: Haushalt, Organisation, Berichtswesen, Statistik, Informationswesen. Ab 1947 nimmt der politische Gehalt des Dokumentationsinhalts deutlich zu, da das Control Office bei der Vereinigung der drei Westzonen und der Vorbereitung der Errichtung eines westdeutschen Teilstaates, der späteren Bundesrepublik Deutschland, eine bedeutende Rolle spielte.

Besonders zu erwähnen sind die Unterlagen der Historical Branch, welche die Aufgabe hatte, Materialien für eine Geschichte der Militärregierung zusammenzustellen; auch wenn diese Tätigkeit nicht immer den uneingeschränkten Beifall Clays fand, enthalten die Unterlagen Schlüsseldokumente zur Geschichte und Tätigkeit der Militärregierung. 


\section{US Political Adviser / Office of Political Affairs}

Die Mehrheit der Überlieferung findet sich nicht in den OMGUS-Unterlagen, sondern in Record Group 84 und in den Akten des amerikanischen Außenministeriums selbst. Dieses Material wurde im OMGUS-Projekt nicht berücksichtigt. Die in Deutschland verfilmt vorliegenden Unterlagen sind insgesamt dennoch für sämtliche historische Fragestellungen durchzusehen, die sich vorwiegend mit auBenpolitischen Problemen auseinandersetzen. Zum Komplex der sog. AuBenpolitik gehört in diesem Falle auch die Vertretung des besiegten Deutschland bzw. der amerikanischen Zone gegenüber Drittstaaten.

\section{Economics Division / Office of the Economics Adviser}

Die in Deutschland verfilmt vorliegenden Unterlagen bieten einen umfangreichen Überblick über die allgemeine und besondere Geschichte der deutschen Wirtschaft, der Wirtschaftsverwaltung und der amerikanisch-deutschen Wirtschaftsbeziehungen. Dabei steht das Problem der Dekartellisierung der deutschen Wirtschaft im Vordergrund. Nicht sauber abzugrenzen ist das Verhältnis der Unterlagen der Economics Division bzw. des Economics Adviser zu den Unterlagen des Bipartite Control Office. $\mathrm{Da}$ der ZusammenschluB der amerikanischen und britischen Besatzungszone zur Bizone im wesentlichen aus wirtschaftspolitischen Überlegungen zustande kam, sind die in diesem Teilbestand zusammengefaßten Unterlagen für wirtschaftshistorische Fragen ebenfalls heranzuziehen.

\section{Finance Division / Office of the Finance Adviser}

Die Unterlagen dokumentieren alle Bereiche der Finanzpolitik einschließlich des Komplexes Geld und Kredit, Zoll, Handelsabkommen und außenwirtschaftliche Fragen. Die unter Allied Bank Commission zusammengefaßten Akten belegen in erster Linie den Wiederaufbau des deutschen Bankensystems, vor allem die Errichtung und Überwachung der Landeszentralbanken, die Währungsreform vom 20. Juni 1948 und die finanzpolitischen Startvoraussetzungen für die Gründung der Bundesrepublik Deutschland.

\section{Manpower Division}

Das Material enthält Unterlagen über die Tarifpartner (Arbeitgeber, Verbände und Gewerkschaften), alle Bereiche der Sozialpolitik einschließlich der Preis- und Lohnpolitik. Hinzu kommen Informationen über die Entnazifizierung insbesondere im $\mathrm{Be}$ reich der Deutschen Arbeitsfront, von Einrichtungen der Sozialversicherung und anderer einschlägiger Einrichtungen. Zum Bereich der Sozialpolitik in diesem Sinne gehört auch der Wohnungsbau. Insgesamt läßt sich feststellen, daß nicht nur die Lage in der amerikanischen, sondern in allen vier Besatzungszonen dokumentiert ist. 


\section{Office of the Director of Intelligence}

Der Schwerpunkt der Überlieferung liegt nicht ausschließlich in Informationen über die amerikanische, sondern insbesondere auch über die sowjetische Besatzungszone Deutschlands. Hervorzuheben sind die Akten der Analysis and Research Branch, die nachrichtendienstliche Analysen über alle Bereiche der Politik enthalten.

\section{Information Services Division}

Die verfilmten Unterlagen dokumentieren in allerdings höchst unterschiedlicher Intensität alle Bereiche der Medienpolitik während der amerikanischen Besatzung Deutschlands. Dabei mögen Unterlagen über die Entwicklung der öffentlichen Meinung in der amerikanischen Zone besonders hervorgehoben werden.

\section{Civil Administration Division}

Unabhängig von der Verwaltungsstruktur, in der Angelegenheiten der allgemeinen inneren Verwaltung wahrgenommen wurden, enthält die Überlieferung reiches Material zu allen Bereichen der Innenpolitik - von der öffentlichen Sicherheit bis zur Wohlfahrtspflege. Hervorzugeben sind die Entnazifizierung und Demokratisierung des deutschen politischen Systems und der öffentlichen Verwaltung in der amerikanischen Zone. Den größten Teil der Überlieferung nehmen die Unterlagen des Director's Office und der Public Safety Branch ein. Erwähnenswert sind ferner die Materialien der für Kriegsgefangene und Displaced Persons zustăndigen Verwaltungseinheiten. Mit den Unterlagen des Control Office vergleichbar sind die Materialien der Civil Administration Division insoweit, als auch hier die Vorbereitung der Errichtung der Bundesrepublik Deutschland umfänglich dokumentiert ist.

\section{Education and Cultural Relations Division}

Die Überlieferung belegt vorwiegend folgende Bereiche: Schul- und Hochschulwesen einschließlich Entnazifizierung, Kirchensachen, Jugend- und Frauenfragen, die Reeducation sowie das Bibliotheks-, Archiv- und Museumswesen, Hinzu kommen Unterlagen über den Kulturaustausch mit den Vereinigten Staaten von Amerika.

\section{Legal Division}

Die Überlieferung ist höchst unvollständig, da Akten der Rechtsabteilung offensichtlich in erheblichem Umfang von HICOG übernommen worden sind. Der Schwerpunkt der Informationen in den verfilmten Beständen liegt bei Fragen der rechtlichen Beratung der Militärregierung, der Justizverwaltung unter besonderer Berücksichtigung der deutschen Justizvollzugsanstalten und der Behandlung von Kriegsverbrechen bzw. Kriegsverbrechern. Die wenigen Akten des OCCWC dokumentieren in erster Linie Fragen der Dienststellenverwaltung der amerikanischen Anklagebehörden 
und der Materialbeschaffung für den Hauptkriegsverbrecherproze $B$ und die zwölf Nachfolgeprozesse in Nürnberg. Die Hauptüberlieferung in diesem Bereich ist in Washington unter Record Group 238 archiviert. Eine im OMGUS-Bestand vorhandene Teilüberlieferung der Special Projects Division beschäftigt sich nicht mit den Nürnberger Prozessen, sondern diente in erster Linie der Vorbereitung künftiger Kriegsverbrecherprozesse vor deutschen Gerichten. 